\title{
The Two Faces of Ascorbate: Pro-oxidant Activity and Radio-sensitisation
}

\author{
By \\ Georgia Carson
}

\begin{abstract}
A thesis
submitted to the Victoria University of Wellington in fulfilment of the requirements for the degree of

Master of Science
\end{abstract}

Victoria University of Wellington

2016 


\section{Acknowledgements}

My greatest, most heartfelt thanks go to Melanie McConnell for trusting me with this project and for always being enormously supportive, wise, creative and friendly. I always felt I could ask her any question and this thesis would never have been completed without her immense help. I will be forever grateful for everything she has taught me.

A huge thanks also to Leticia Castro for her in vitro technical training and hep with trouble-shooting, and for putting up with my countless questions. Leticia taught me everything I needed to know about cell culture and flow cytometry assays.

Big thanks to Carole Grasso and Cameron Field at the Malaghan Institute of Medical Research, who were invaluable in teaching me all the in vivo work. Cam and Carole showed me intracranial surgery, mouse care and handling, and how to go about harvesting tumours. Big thanks also to the Malaghan Biomedical Research Unit staff who took great care of my mice and made my animal work run smoothly.

I am indebted to those at Victoria University who gave me advice and lent me reagents along the way: Pirooz Zareie for training me on the flow cytometer, Nikki Templeton for the training and use of histology solutions and equipment, Varun Venkatesh for the SLS and IF tips, and Lesley Milicich for Sudan Black.

Thank you also to the whole of the MMC group for being so supportive and making me feel part of the family: Mel, Leticia, MarieSophie, Nicole, Remy, Dini, Matt, Dan, Dana, Kristal and Billy.

I owe my sanity to the joke-cracking office Space Station class of 2015L Pirooz, Matt, Sarah, Vimal and Nikki, who never failed to bring laughter to the long days. Thanks guys.

A lot of appreciation goes to my non-university family and friends, who were always understanding about my long hours, and failure to return calls promptly. To Mum and Richard, and Dad and Donna, thanks for the free meals; to Hannah and Miranda, best flatmates ever; thanks Hugo for semi-learning how to cook and hugs; and thanks to Jess for the coffee beans while I was writing - much appreciated! 
Although not recommended by mainstream oncologists, intravenous injections of pharmacological ascorbate are currently an alternative therapy option for cancer patients. Research has not yet determined whether highdose ascorbate interacts favourably with radiation therapy to increase DNA damage, and therefore cell death in cancer. Some studies suggest that ascorbate can act as a prooxidant and increase the cytotoxic effect of irradiation in vitro. Glioblastoma multiforme $(\mathrm{GBM})$ is a primary brain astrocytoma that is highly therapy resistant, so patients would be advantaged if ascorbate radiosensitised their cancer.

In this investigation, flow cytometry and single cell gel electrophoresis (comet tail assay) were used to measure three indicators of DNA damage in GBM cells in response to ascorbate and irradiation, and were contrasted with immunofluorescence-revealed DNA damage from an intracranial mouse model of GBM.

The pro-oxidant, radiosensitisation role of ascorbate was confirmed, as measured by $\Upsilon \mathrm{H} 2 \mathrm{AX}, 8 \mathrm{OHdG}$, and DSBs in vitro. With all three of these markers of DNA damage, combinations of irradiation and ascorbate had increased damage compared with individual treatments. However preliminary in vivo evidence indicates that increased DNA damage did not occur in an animal model of GBM, and in fact ascorbate may protect from DNA damage in an in vivo context.

These findings complement previous results from our lab, and serve to fill in gaps in knowledge specifically around the DNA damaging effects of ascorbate. The unique nature of the brain environment, as enclosed by the blood brain barrier, prevents translation of data from other non-brain cancer studies, as such, this investigation also contributes to the exploration of a much needed avenue of research. Considering the context of ascorbate treatment as a potentially harmful currently used adjuvant, it is imperative to confirm or disprove its efficacy in a clinically relevant environment. 


\section{Table of Contents}

Introduction ................................................................................................................

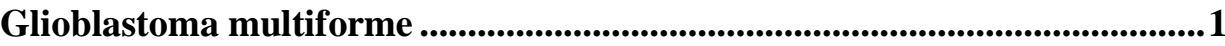

GL261 Mouse Model of GBM ................................................................. 4

Markers of DNA Damage.................................................................5

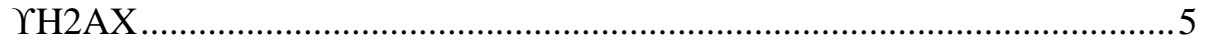

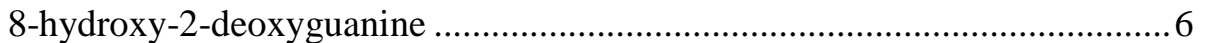

Comet Tail Assay ............................................................................. 7

Radiation .............................................................................................9

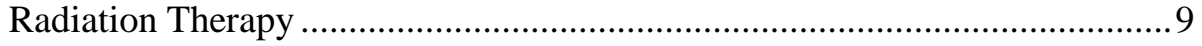

DNA Damaging Effects of Radiation.................................................... 11

Ascorbate ..........................................................................................14

Ascorbate Basic Facts ........................................................................ 14

Ascorbate as an Antioxidant................................................................... 19

Ascorbate as a Co-factor ................................................................ 22

Ascorbate as a Prooxidant: The Fenton and Haber-Weiss reaction ............... 28

Ascorbate Transport .............................................................................. 30

Ascorbate Pharmacokinetics ................................................................... 33

Ascorbate as Disease Therapy ................................................................. 36

Ascorbate as Cancer Therapy .............................................................. 37

Ascorbate as a Prooxidant Cancer Therapy ....................................... 44

Aims ....................................................................................................53

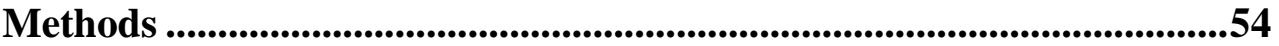

Materials ..........................................................................................................54

Cell Lines .............................................................................................................54

Cell Culture .........................................................................................................54

Media ....................................................................................................................54

Treatments...........................................................................55

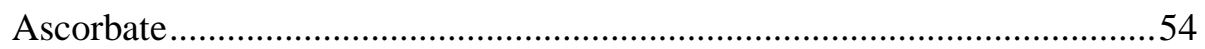

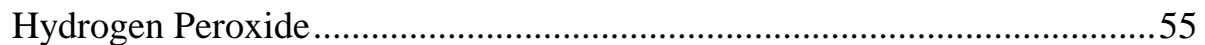

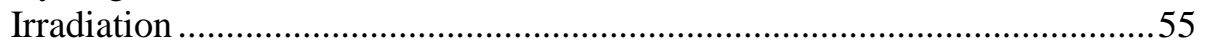

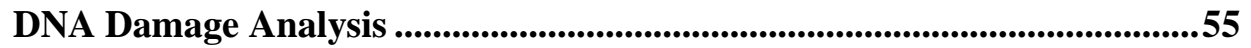

Flow Cytometry................................................................................. 55

Comet Tail Assay/Single Cell Gel Electrophoresis.....................................57

Intracranial Mouse Model ............................................................................59

Whole Brain Irradiation of Mice............................................................63

Tumour Harvest...................................................................................63

Tissue Processing and Immunofluorescence Assay ..................................63

Statistical Analysis ...................................................................65

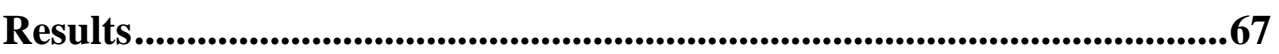

Analysis of DNA Damage In Vitro .........................................................67

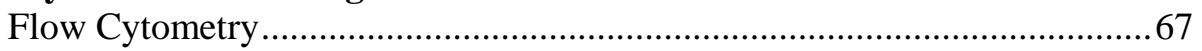

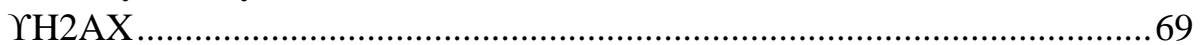

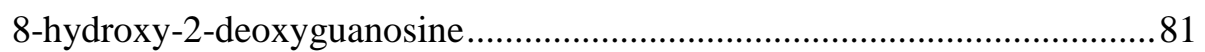

Single Cell Gel Electrophoresis/Comet Tail Assay .................................... 91

Analysis of DNA Damage In Vivo ..............................................................109

In Vivo Experimental Outline .............................................................. 109

Immunofluorescence Assay.......................................................... 113

Chapter 4: Discussion.....................................................................133

In Vitro .........................................................................................................133

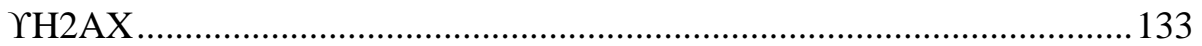




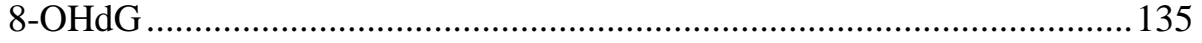

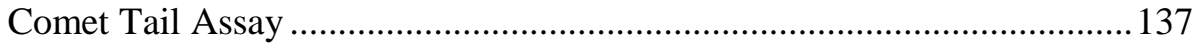

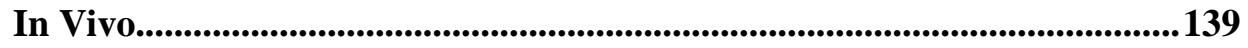

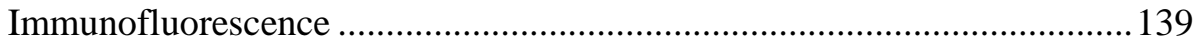

Confounding Factors .............................................................................................140

Selectivity .....................................................................................................................142

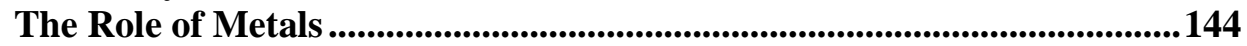

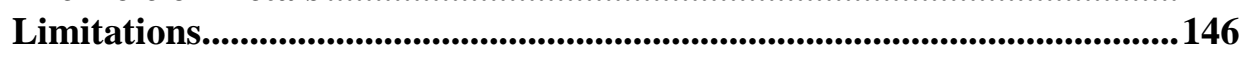

Future Experiments .................................................................................148

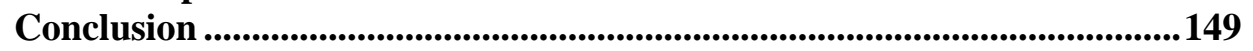

List of Abbreviations ............................................................................169 


\section{Table of Figures}

Figure 1 Biosynthesis of ascorbate

Figure 2 Stereochemical isomers L-Ascorbic Acid (L), D-Ascorbic Acid

(R)

Figure 3. The Redox forms of Ascorbate .................................................... 18

Figure 4. Resonance Stabilisation of the Ascorbyl Radical .......................21

Figure 5. Ascorbate acts as a co-factor in the hydroxylation of proline in the biosynthesis of collagen

Figure 6. Injection site of GBM cells in the mouse skull. Red X represents site of injection.

Figure 7. A graphical representation of whole brain mouse irradiation ......66

Figure 8. Example representative analysis of flow cytometry cell data ......68

Figure 9. Representative example of YH2AX analysis in GL261 cells in

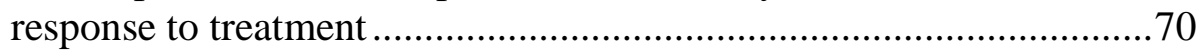

Figure 10. Flow cytometry derived percent positive $\mathrm{YH} 2 \mathrm{AX}$ staining in GL261 cells

Figure 11. Flow cytometry derived median fluorescence intensity $\mathrm{rH} 2 \mathrm{AX}$ staining in GL261 cells.

Figure 12. Flow cytometry derived percent positive $\mathrm{YH} 2 \mathrm{AX}$ staining in T98G cells

Figure 13. Flow cytometry derived median fluorescence intensity $\mathrm{rH} 2 \mathrm{AX}$ staining in $\mathrm{T} 98 \mathrm{G}$ cells

Figure 14. Flow cytometry derived percent positive $\mathrm{rH} 2 \mathrm{AX}$ staining in 1003 cells

Figure 15. Flow cytometry derived median fluorescence intensity $\mathrm{\Upsilon H} 2 \mathrm{AX}$ staining in 1003 cells

Figure 16. Flow cytometry derived percent positive 8-hydroxy-2deoxyguanosine staining in GL261 cells

Figure 17. Flow cytometry derived median fluorescence intensity 8hydroxy-2-deoxyguanosine staining in GL261 cells

Figure 18. Flow cytometry derived percent positive 8-hydroxy-2deoxyguanosine staining in $\mathrm{T} 98 \mathrm{G}$ cells

Figure 19. Flow cytometry derived median fluorescence intensity 8hydroxy-2-deoxyguanosine staining in $\mathrm{T} 98 \mathrm{G}$ cells

Figure 20. Flow cytometry derived percent positive 8-hydroxy-2deoxyguanosine staining in 1003 cells ....

Figure 21 . Flow cytometry derived median fluorescence intensity 8 hydroxy-2-deoxyguanosine staining in 1003 cells

Figure 22. Graphical representative analysis of comet tails using the ImageJ comet tail plugin

Figure 23. Example display of typical comet nuclei in response to either no treatment (L) or 80 Gy of irradiation (R)

Figure 24. Single cell gel electrophoresis ascorbate time course tail lengths

Figure 25. Single cell gel electrophoresis ascorbate time course tail moments

Figure 26. Single cell gel electrophoresis ascorbate time course percent tail DNA 
Figure 27. Single cell gel electrophoresis irradiation dose response tail lengths 101

Figure 28. Single cell gel electrophoresis irradiation dose response tail moments.

Figure 29. Single cell gel electrophoresis irradiation dose response percent tail DNA

Figure 30. Single cell gel electrophoresis combination treatment tail lengths

Figure 31. Single cell gel electrophoresis combination treatment tail moments

Figure 32. Single cell gel electrophoresis combination treatment percent tail DNA

Figure 33. In Vivo Experimental Plan.

Figure 34. Experimental mouse weights

Figure 35. Normal, untreated brain tissue has little $\mathrm{rH} 2 \mathrm{AX}$ positive staining

Figure 36. Normal, irradiated brain tissue has an increase in basal $\mathrm{rH} 2 \mathrm{AX}$ positive staining.

Figure 37. Representative Images of DAPI stained tumour tissue from untreated brain.

Figure 38. Representative Images of $\mathrm{YH}_{2} \mathrm{AX}$ and DAPI stained tumour tissue from untreated brain

Figure 39. Representative Images of $\mathrm{YH} 2 \mathrm{AX}$ and DAPI stained tumour tissue from ascorbate treated brain

Figure 40. Representative Images of $\Upsilon \mathrm{H} 2 \mathrm{AX}$ and DAPI stained tumour tissue from irradiated brain.

Figure 41. Representative Images of $\Upsilon \mathrm{H} 2 \mathrm{AX}$ and DAPI stained tumour tissue from ascorbate pre-treated, irradiated brain

Figure 42. Representative Images of $\mathrm{YH} 2 \mathrm{AX}$ and DAPI stained tumour tissue from irradiated, ascorbate post-treated brain

Figure 43. Representative Images of 8-OHdG and DAPI stained tumour tissue from untreated brain

Figure 44. Representative Images of 8-OHdG and DAPI stained tumour tissue from ascorbate treated brain

Figure 45. Representative Images of 8-OHdG and DAPI stained tumour tissue from irradiation treated brain.

Figure 46. Representative Images of 8-OHdG and DAPI stained tumour tissue from ascorbate pre-treated, irradiated brain

Figure 47. Representative Images of 8-OHdG and DAPI stained tumour tissue from irradiated, ascorbate post-treated brain 


\section{Table of Tables}

Table 1. Types and Frequencies of Cell Damage from Irradiation 12

Table 2. Ascorbate Transporters.

Table 3. Outputs of the ImageJ Comet Tail Plugin ....................................59

Table 4. Automated Tissue Processor Program ...........................................64

Table 5. Immunofluorescence Protocol ........................................................64

Table 6. Optimisation of Immunofluorescence Assay 


\section{Introduction}

Pharmacological levels of ascorbate, applied via intravenous injection, are currently an alternative therapy option for cancer patients. This treatment is rationalised by in vitro evidence that demonstrates ascorbate acts as a pro-oxidant. Although ascorbate is not part of the recommended course of therapeutics by mainstream oncologists, the ease of access and the number of alternative clinics that offer the treatment indicates many patients have almost certainly already integrated it with traditional radiation and chemotherapy. However, research has not yet determined whether high-dose ascorbate interacts favourably with radiation therapy in order to increase DNA damage and therefore cell death in cancerous cells. Therefore, there is a strong imperative to fully investigate the consequences of ascorbate in combination with radiation, and to determine whether its influence is beneficial, or, potentially, harmful, particularly in glioblastoma brain tumours, which are otherwise therapy resistant. The investigation detailed here confirms radiosensitisation of GBM cells in vitro by ascorbate, but a potential radioprotective role for ascorbate in vivo.

\section{Glioblastoma multiforme}

Glioblastoma multiforme (GBM) is the most common and malignant primary brain neoplasm. Derived from astrocytes, it is responsible for $70 \%$ of all malignant primary brain tumours (Wen and Kesari 2008) and 82\% of malignant gliomas (Omuro and DeAngelis 2013). Incidence is estimated at approximately 3 per 100,000 adults per year (Ohgaki and Kleihues 2005; Urbanska, Sokolowska et al. 2014), although this varies by nation (Bondy, Scheurer et al. 2008; Omuro and DeAngelis 2013; Wen and Kesari 2008). The cancer is more common in males and Caucasians (Chinot 2009) and the average age of incidence is 62 years (Ohgaki and Kleihues 2005). 
The term glioblastoma multiforme was coined in 1926 by Harvey Cushing and Percival Bailey; glioblastoma referencing the cancers glial, astrocytoma origin, while multiforme alludes to the heterogeneity caused by foci of necrosis and haemorrhage present in this variety of tumour (Greenberg, Chandler et al. 1999; Urbanska, Sokolowska et al. 2014). The global authority on health taxonomy, the World Health Organisation (WHO) classes glioblastoma multiforme (which the WHO now simply calls glioblastoma) as a grade four astrocytoma. This is judged by factors such as atypia, morphological differentiation, and degree of vascularisation and necrosis. Grades correspond to the malignancy of tumours such that GBM is the one of the most malignant, as opposed to pilocytic astrocytomas, diffuse astrocytomas, and anaplastic astrocytomas; grades one to three respectively (Louis, Ohgaki et al. 2007).

There is limited conclusive evidence on the causes of GBM (Alifieris and Trafalis 2015; Omuro and DeAngelis 2013; Wrensch, Minn et al. 2002), however there are several studies that show ionising radiation as a significant risk factor for tumours of the central nervous system (CNS). For example, there is a greater prevalence of gliomas in patients that have received therapeutic radiation in the past for conditions such as ALL (acute lymphoblastic leukaemia) (Salvati, Artico et al. 1990), and survivors of the atomic bombing in Hiroshima showed a greater prevalence of meningioma (tumours of the meninges) that correlated with their distance from the epicentre (Shintani, Hayakawa et al. 1999). A small proportion of highgrade gliomas can be explained by hereditary conditions such as LiFraumini syndrome (a mutation of the TP53 tumour suppressor gene), neurofibromatosis type 1 (a mutation of the NF1 gene that negatively regulates the Ras pathway), or Turcot's syndrome (mutations in DNA mismatch repair) (Chinot 2009; Wen, Fine et al. 1995). Furthermore, environmental exposure to certain metals, solvents or chemicals, especially those utilised in the petroleum and rubber processing industries, have been implicated in glioma (Spinelli, Chinot et al. 2010; Wrensch, Minn et al. 2002). 
There are a variety of oncogenic changes, and different combinations of these changes, that produce GBM (for a detailed review see Crespo, Vital et al. 2015). Although heterogeneous, gene alterations have been associated with GBM progression, such as p53 mutations, deletions in chromosome 10, epigenetic changes in epidermal growth factor receptor (EGFR) (Ohgaki, Dessen et al. 2004), and mutations in the genes for platelet-derived growth factor (PDGF) receptor and ligands (Verhaak, Hoadley et al. 2010), cyclin-dependent protein kinases (CDKs) (Nakamura, Watanabe et al. 2001), phosphatase and tensin homolog (PTEN) (Ohgaki, Dessen et al. 2004), and O6-methylguanine-DNA methyl transferase (MGMT) (Nakamura, Watanabe et al. 2001). Epigenetic silencing of MGMT is a marker of therapy responsiveness and longer survival in gliomas (Chinot 2009; Esteller, Garcia-Foncillas et al. 2000).

GBM can be either primary or secondary. Primary cancers arise de novo and are most common in older adults. They are generally aggressive and have lower survival rates (Porter 2012). The vast majority (90\%) of GBM is of this type (Urbanska, Sokolowska et al. 2014). Secondary cancers progress from lower grade tumours and thus develop over a longer time (45 years) than primary tumours (Ohgaki and Kleihues 2013, Urbanska, Sokolowska et al. 2014). Although primary and secondary GBM are morphologically alike, they are genetically distinct and require a different therapeutic response (Crespo, Vital et al. 2015; Ohgaki and Kleihues 2013; Urbanska, Sokolowska et al. 2014).

GBM is highly resistant to therapy, partly due to its invasive and heterogeneous nature (Wen and Kesari 2008). It is suggested that a subpopulation of cancer stem cells contributes to GBMs strong therapy resistance (Huang, Cheng et al. 2010), as well as hypoxia-induced cell survival factors and hypoxic inhibition of radiation (Legendre and Garcion 2015). Despite a suite of treatments available, including surgery, radiation and chemotherapy, prognosis for GBM remains poor. Median survival is only 14 months, 1 -year survival is less than $40 \%$, and the 5 -year survival is less than 5\% (Omuro and DeAngelis 2013). Some population wide analyses 
have observed even lower survival for GBM (Ohgaki and Kleihues 2005). This prognosis has improved minimally in the past decades, despite an increase in the variety of therapies (Huang, Cheng et al. 2010).

There are various proposed treatments for GBM that are under development, including immunotherapies such as bevacizumab (anti-VEGF antibody) (Omuro and DeAngelis 2013), anti-VEGF gene therapy, blockade of NHERF-1 synthesis, and inhibition of the Mer tyrosine kinase receptor (Urbanska, Sokolowska et al. 2014).

\section{GL261 Mouse Model of GBM}

The GL261 cell line is representative of a carcinogen-induced mouse syngeneic glioma model (Newcomb and Zagzag 2009). Syngeneic models are particularly valuable to study tumour growth in vivo because, unlike the human xenograft models, the syngeneic models do not require a deficient immune system and may mimic more closely the interaction between tumour and immune system that takes place in human GBM patients. For example, in vivo tumours arising from the GL261 cell line have irregular, invasive borders similar to spontaneous human GBM tumours, rather than the clearly defined edges of xenograft, human glioma U87MG created tumours (Newcomb and Zagzag 2009).

Seligman and Shear (1939) originally developed what would become GL261. Cells were derived from invasive gliomas of C3H mice that developed in response to pellets of 20-methylcholanthrene inserted in the cortex.

Later, Ausman et al. (1970) propagated the line by inserting pieces of the original tumour into C7BL6 mice. They found GL261 to be an effective model for an aggressive murine glioma, with animals succumbing reproducibly after around 24 days subsequent to intracranial implantation. This study characterised the tumours based on histopathology as ependymoblastoma, although later investigations altered this classification to most similar to GBM (Newcomb and Zagzag 2009). 
During the 1990s, several labs reinforced this work by forming a permanent cell line from the gliomas (Szatmari, Lumniczky et al. 2006). Szatmari et al. did a thorough characterisation of the cell line, and discovered point mutations in the K-ras and p53 genes and expression of basal major histocompatibility complex (MHC) 1. GL261 was radiosensitive: less than 2 Gy killed half the cells in vitro and 4 Gy in mice slowed the progression of tumours. It was also immunogenic: vaccination of mice with irradiated GL261 cells 7 days before intracranial tumour injection inhibited cancer development in nearly all mice (Szatmari, Lumniczky et al. 2006).

GL261 has been used extensively in vivo (and as an in vitro comparison), especially for pre-clinical tests (Burgi, Seuwen et al. 2014; Miyatake, Martuza et al. 1997; Newcomb and Zagzag 2009; Plautz, Touhalisky et al. 1997; Szatmari, Lumniczky et al. 2006), with nearly 40 papers published in the last year alone utilising the model (for example Mathios 2016; Zhao, Jacobs et al. 2015).

\section{Markers of DNA Damage}

\section{$\Upsilon \mathrm{H} 2 \mathrm{AX}$}

$\mathrm{H} 2 \mathrm{AX}$ is a highly conserved, $14 \mathrm{kD}$ histone of the $\mathrm{H} 2 \mathrm{~A}$ family, one of the five major histone families (as well as $\mathrm{H} 2 \mathrm{~A}$, these are $\mathrm{H} 1 / \mathrm{H} 5, \mathrm{H} 2 \mathrm{~B}$, $\mathrm{H} 3$ and $\mathrm{H} 4)$. $\mathrm{H} 2 \mathrm{AX}$ plays a major role in the $\mathrm{H} 2 \mathrm{~A}$ family, composing up to a quarter of the mammalian histone H2A's, although this varies in different cell lines and tissues; as well as being crucial for recombination among immunoglobulin switch regions. The protein is a basal histone, thus synthesised during the G1 and S phase of the cell cycle (Pouliliou and Koukourakis 2014; Redon, Pilch et al. 2002).

The phosphorylation of H2AX, $\mathrm{rH} 2 \mathrm{AX}$ is a commonly used indicator of double-stranded DNA breaks, as its modification is an intrinsic part of the DNA repair pathway (Pouliliou and Koukourakis 2014). It has been employed in vitro to investigate fundamental DNA repair, and in vivo 
to study response to diagnostic irradiation (Beels, Bacher et al. 2009), as well as clinical response to chemotherapy and therapeutic irradiation (Ivashkevich, Redon et al. 2012).

Traditional thought is that following DSB formation, phosphoinositide 3-kinase-like (PI3) proteins, especially ATM, quickly cause phosphorylation of the histone H2AX C-terminal tail at serine 139. This causes various downstream effects designed to enhance DNA repair, for instance, recruitment of damage sensors and repair proteins to the region of damage, amplification of the DNA damage response signal to ensure cell cycle checkpoint factors are activated, and chromatin remodelling such that DNA repair enzymes can access the damage more easily (Podhorecka, Skladanowski et al. 2010).

Although it has been shown to be the case that $\mathrm{H} 2 \mathrm{AX}$ is phosphorylated in response to DSBs (Rogakou, Pilch et al. 1998), for a number of years literature has referred to $\mathrm{rH} 2 \mathrm{AX}$ less as a marker specifically for double stranded breaks, and more DNA breaks, DNA damage response (Brumbaugh, Otterness et al. 2004; Hardee, Marciscano et al. 2012; Yuan, Adamski et al. 2010) or for generic genotoxic damage and stress (Morris, Boutell et al. 2009; Tanaka, Kurose et al. 2006). There is thus some doubt on whether $\mathrm{\Upsilon H} 2 \mathrm{AX}$ is specific only for double-stranded breaks. The modification is an important part of the DNA damage response, but it remains possible that $\mathrm{YH} 2 \mathrm{AX}$ could be induced in response to other cellular events such as chromatin modelling, stalled replication, or heat stress (Takahashi and Ohnishi 2005; Yuan, Adamski et al. 2010). It may eventually be determined that these events do activate $\mathrm{YH} 2 \mathrm{AX}$ via DSBs, but this is yet to be clarified. In any case, it is prudent to use a variety of methods to further validate the presence of DNA damage varieties.

\section{8-hydroxy-2-deoxyguanine}

8-hydroxy-2-deoxyguanine (8-OHdG) is a modified purine, a type of DNA lesion caused by oxidative damage. Oxidative stress occurs at every moment in all cells, and because repair mechanisms are not 
completely effective, markers of stress increase with age. $8-\mathrm{OHdG}$ is thought to be the most mutagenic and widespread of these markers (Valavanidis, Vlachogianni et al. 2009). It is often used as a proxy for DNA damage, most commonly as a urine biomarker for general oxidative stress. For example, human studies have used the marker to measure effects of carcinogens such as tobacco and asbestos (Pilger and Rudiger 2006). In monocytes, the rate of 8-OhdG creation has been measured as in the region of 20 per $10^{9}$ bases per Gy as a result of radiation (Sonntag 2006).

Current estimates of steady-state levels are less than 100-1000 8hydroxy 2-deoxyguanosine residues in normal cells (Klungland, Rosewell et al. 1999). It is important to remember, however, that while oxidized DNA bases will impair DNA function, such bases always exist at some basal level, and cells have numerous repair systems to remove such species (Lindahl and Wood 1999). However, if they occur at critical sites, or are not quickly repaired, oxidized purines or pyrimidines can cause functional problems. As a result, oxidized DNA bases are considered an important event in chemical carcinogenesis (Klaunig, Xu et al. 1998).

\section{Comet Tail Assay}

The comet tail assay is a technique that determines the extent of cellular DNA damage, by measuring the distance DNA fragments travel from the nucleus under electrophoresis. It is so called because of the comet shape that the nucleoids form subsequent to application of an electric charge. Also known as single cell gel electrophoresis (SGE) or microgel electrophoresis (MGE), it was first developed by Ostling and Johanson in 1984 to directly visualise DNA damage at the scale of individual irradiated cells (Ostling and Johanson 1984).

Briefly, single cell suspensions are diluted in low melting point agarose, set on glass slides, lysed, electrophoresed, and stained for imaging. The rationale behind this process is that the degree of damage for each cell corresponds with parameters such as the length of the DNA 'tail' that trials behind the nucleoid, and the percentage of DNA in the tail; because relaxed 
or damaged DNA travels further than wound and intact DNA. Ostling and Johanson determined the amount of DNA that extended out from the comet's 'head' was proportional to the dose of radiation that cell received.

There are two major categories of comet assay, the alkaline and the neutral. The initial experiments by Ostling and Johanson used neutral lysis conditions, which only allow detection of double strand breaks (DSBs). This is because under neutral $\mathrm{pH}$, DNA base pairing is maintained, eliminating observation of gaps in single DNA strands. On the other hand, observation of single stranded breaks (SSBs) needs alkaline conditions $(\mathrm{pH}>12.3)$ that denature and unwind DNA strands (Liao, McNutt et al. 2009). This alteration to the assay was developed a short time later (Singh, McCoy et al. 1988) and is now the most commonly used. Additionally, some sites, known as alkaline labile lesions, only become strand breaks, and so able to be detected, in the presence of alkali (Fairbairn and O'Neill 1995).

A strong advantage of this assay is that damage can be measured in individual cells, so heterogeneous responses can be identified. This is useful in, for example, viewing subpopulations of cells that may be unusually resistant compared to the whole population; other assays, such as the alkaline unwinding assay, only display an average damage measure. Other advantages include sensitivity, the requirement for low cell numbers, flexibility that means the comet assay can be applied to nearly all eukaryotic cells, and no need for specific, costly antibodies (Huycke 2003; Nishimura 2006; Speit and Hartmann 2006).

The comet tail assay is frequently used to establish the genotoxicity of environmental DNA damaging agents. For example, smoking (Hoffmann, Hogel et al. 2005), hydrogen peroxide (Driessens, Versteyhe et al. 2009), environmental radiation (Tice and Strauss 1995) and other carcinogens (Zeljezic, Mladinic et al. 2015). The assay can also be used to ascertain cells response to anti-cancer agents such as radiotherapy or bleomycin. (Ostling and Johanson 1984). A study even used the comet tail 
assay to look at the prooxidant effects of ascorbate and copper in lymphocytes (Bhat, Azmi et al. 2006).

\section{Radiation}

\section{Radiation Therapy}

GBM is characterised by an infiltrating tumour mass that precludes clear differentiation from regular tissue and complete removal during surgery (Karcher, Steiner et al. 2006). Thus, as with other solid tumours, the standard treatment for GBM is surgery, followed by radiation therapy (Simpson, Horton et al. 1993), with a chemotherapy such as temozolomide as an adjuvant (Stupp, Mason et al. 2005).

Radiation therapy is controlled energy released from the decay of a radioactive source, targeted to malignant regions in an attempt to eliminate unrestricted growth. Radiation sources for therapy generally include isotopes such as cobalt 60 or caesium 137 (Woods and Pikaev 1994). The radiation is ionising, that is, the energy is great enough that it can remove electrons from atoms, including those that comprise the DNA molecule. When this damage from irradiation becomes so great it is irreparable, the cell dies (Eriksson and Stigbrand 2010).

Patients usually receive thirty fractions of 1.8-2.0 Gray (Gy) to a total of 60 Gy (Chinot 2009; Omuro and DeAngelis 2013) over around 3 to 8 weeks (Withers 1992). 1 Gray corresponds to 1 joule per kilogram of mass that absorbs the radiation (Bureau International des Poids et Mesures 2014).

Before the advent of irradiation as therapy, there was evidence that irradiation could interfere with cellular function and survival. In the 1940s Euler, Hevesy, and Ahlström observed after irradiation, tagged precursors had inhibited incorporation into DNA, indicating irradiation prevents DNA synthesis (Barnum, Scheller et al. 1964). 
Until the discovery of effective chemotherapy, irradiation was the only treatment that could lengthen survival in patients with high-grade gliomas (Chinot 2009). The effectiveness of radiation in brain cancer was first demonstrated by Walker et al., who extended average survival from 14 weeks to 36 weeks by use of brain radiotherapy in high-grade gliomas. (Walker, Eben Alexander et al. 1978).

Naturally, such a powerful treatment as irradiation has some adverse effects. Daily dose, total dose, and the amount of brain irradiated are factors that influence risk (Sheline, Wara et al. 1980). It was subsequently discovered that doses greater than 60 Gray unacceptably increased the damage to normal brain tissue (Leibel, Scott et al. 1991), accordingly a cap of 60 Gray is the norm (Wen and Kesari 2008). Furthermore, originally irradiation was applied to the whole brain to improve chances of eliminating tumours, however this fell out of favour when it was discovered that whole brain irradiation impaired brain function (Lee, Cho et al. 2012). Currently, irradiation is conserved to the tumour regions as much as possible by methods such as brachytherapy and individually shaped irradiation beams that minimise damage to normal tissue.

Even when used correctly, irradiation causes side effects. Acute, delayed and late toxicity effects appear during, 2 to 3 months after, and over 1 year after irradiation therapy respectively (Chinot 2009). Acute effects are thought to be caused by oedema, delayed effects by demyelination, and late effects by alteration to the brain's vascular system (Sheline, Wara et al. 1980). An example of late toxicity is radiation necrosis, which has an overall incidence of approximately $4.9 \%$ (Ruben, Dally et al. 2006) Radiation necrosis is injury to the peritumoural white matter, and is characterised by cell necrosis, vascular permeability and endothelial cell apoptosis, that leads to seizures and sometimes death (Siu, Wind et al. 2012). 


\section{DNA Damaging Effects of Radiation}

Experiments have shown that cytotoxicity is determined by the dose received by, and damage incurred by the nucleus, as opposed to the membrane or cytoplasm. Different types of irradiation that selectively dose the cytoplasm/outer membrane, nucleus, or the entire cell, indicate that irrespective of irradiation received by the non-nucleus regions, a nuclear dose of between 3 and 4 Gy is sufficient to induce LD50 levels of cell death (Sonntag 2006).

Irradiation damage can be either indirect or direct, by ionising the water in the vicinity of the nucleic acid, or ionising the strand itself (Sonntag 2006).

Indirect damage of DNA by radiolysis of the water molecules surrounding it is mediated by creation of the free radicals $\mathrm{OH}, \mathrm{H} \cdot$ and $\mathrm{e}_{\mathrm{aq}}{ }^{-}$. These reactive species can only influence DNA if they are in close proximity, 2 Angstroms $\left(\mathrm{A}^{\circ}\right)$ or fewer, for they can easily be scavenged by endogenous agents (Sonntag 2006).

The direct effect of ionising radiation also contributes to damage DNA. In this case, when the irradiation reaches DNA it can ionise the actual DNA molecule, producing a DNA radical cation (DNA-+) and an electron, in addition to electronically excited DNA. This contributes to amplification effects that further promote damage (Sonntag 2006).

Via indirect or direct means, ionising radiation causes a variety of types of damage, yet not all forms are created to the same degree in a given cell with a given dose (Table 1). 
Table 1. Types and Frequencies of Cell Damage from Irradiation

\begin{tabular}{|l|l|}
\hline Immediate Physical Events & \\
\hline Ionizations in the cell nucleus & $\sim 100,000$ \\
\hline Ionizations directly in DNA & $\sim 2000$ \\
\hline Excitations directly in DNA & $\sim 2000$ \\
\hline DNA Damage Effects & \\
\hline SSBs & 1000 \\
\hline $\begin{array}{l}\text { 8-oxo-A (a typical single-base } \\
\text { damage) }\end{array}$ & 700 \\
\hline DSBs & 40 \\
\hline DNA-protein cross-links & 150 \\
\hline Whole cell effects & \\
\hline Lethal events & $\sim 0.2-0.8$ \\
\hline Chromosome aberrations & $\sim 1$ \\
\hline Hprt mutations & $\sim 10-5$ \\
\hline $\begin{array}{l}\text { In response to 1 Gy radiation. } \\
\text { From 'Free Radical Induced DNA and Its Repair' (Sonntag 2006) }\end{array}$ \\
\hline
\end{tabular}


In themselves, individual lesions are not necessarily lethal to a cell. Even the most severe alteration, a double-stranded break, is not sufficient to induce cytotoxicity, despite a higher risk of misrepair because of lack of a template strand (Withers 1992). For example, it has been shown that an LD50 (50\% lethal dose) in mammalian cells will produce 150 single stranded breaks (SSBs) and 30 double stranded breaks (DSBs), which demonstrates the average level of damage that can be dealt with by cellular repair mechanisms (Sonntag 2006). However, when this damage surpasses what the cell can repair, apoptosis occurs and the cell dies.

DNA damage from radiation selectively affects cancerous cells because they reproduce more quickly, and with less quality control, than most normal cells. This rapid proliferation gives less opportunity for repair of sub-lethal damage, and so mutations are more likely to accumulate (Withers 1992). Cancer cells also have limited DNA repair capacity in relation to regular cells, because often genes associated with the DNA damage response (DDR) are mutated (Ciriello, Miller et al. 2013), prompting positive feedback where cells become more mutated (Jackson and Bartek 2009).

However, the selectivity is not absolute or else GBM could be eliminated entirely with a supremely large dose of irradiation carefully applied to the correct area. Irradiation dosages that are capable of irradiating all cancerous cells are so large as to irreparably harm healthy tissue (Withers 1992). Additionally, there are various factors such as hypoxia which further alter radio-sensitivity. This fact has prompted much work to investigate potential radio-sensitisers that improve cancer cells vulnerability to irradiation while minimising effect on non-cancerous cells.

One of those potential radiation sensitisers is ascorbate. 


\section{Ascorbate}

\section{Ascorbate Basic Facts}

Ascorbate is more commonly known as vitamin C. Vitamins are naturally occurring organic molecules that the human body requires to function. Most have to be acquired from the environment, as they cannot be synthesised by humans (Australian Government Department of Health and Ageing National Health and Medical Research Council and New Zealand Ministry of Health 2006; Bendich and Deckelbaum 2015).

Vitamins are either fat soluble, such as Vitamin A, D, and K, or water soluble, such as Vitamin B and C. Fat soluble vitamins can be stored in the liver or fatty tissue, but water soluble vitamins are excreted rapidly and need to be replaced (Bendich and Deckelbaum 2015).

Such is the case with vitamin C. It is found in many fruits and vegetables (Australian Government Department of Health and Ageing National Health and Medical Research Council and New Zealand Ministry of Health 2006) and is an essential vitamin for humans, as it is an important co-factor in various biochemical reactions (Linster and Van Schaftingen 2007). Humans have lost the ability to synthesise it (Burns 1957). Unlike most animals, Homo sapiens have no functional L-gulomo-Y-lactone oxidase (GLO) gene, but instead a highly mutated pseudogene. A primate sub-order (Pollock and Mullin 1987), guinea pigs and most bats have also lost the ability to synthesise ascorbate, and thus develop scurvy from ascorbate inadequate diets. 


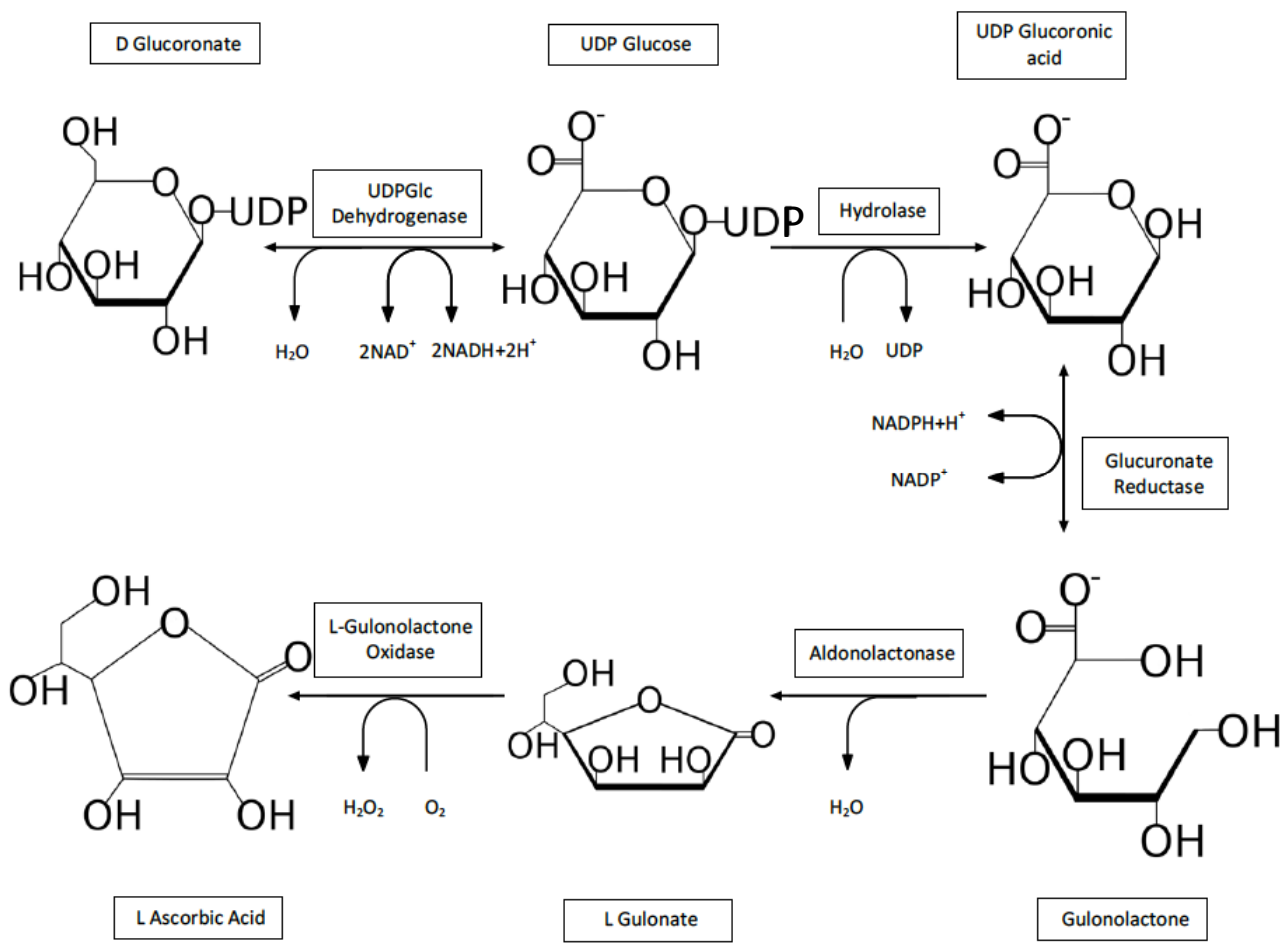

Figure 1 Biosynthesis of ascorbate

Adapted from (Banhegyi, Braun et al. 1997) 
Other animals that can synthesise ascorbate via GLO convert glucose to L-ascorbate in the liver by the mechanism in Figure 1. The ' $\mathrm{L}$ ' nomenclature of 'L-ascorbic acid' is an indication of the molecule's stereochemistry (Figure 2). D-ascorbic acid, unlike L-ascorbic acid, is not found in nature, and lacks most of the vitamin $\mathrm{C}$ activity. Unless specified, the term ascorbate should be assumed to be 'L-ascorbic acid' (Crawford 1982).

Vitamin $\mathrm{C}$ is the general synonym for the related group of molecules that possess vitamin activity, that is, can prevent scurvy. (Australian Government Department of Health and Ageing National Health and Medical Research Council and New Zealand Ministry of Health 2006). These molecules, known as 'vitamers' differ depending on redox form, determinant on pH. (Domitrović 2006).

The most reduced member of the group is ascorbic acid; however, at physiological $\mathrm{pH}$, ascorbate, or the ascorbate monoanion, is dominant, owing to a first and second pKa of 4.2 and 11.6 respectively (Domitrović 2006). When oxidised, the anion becomes an ascorbate radical, while further oxidation converts the compound to dehydroascorbic acid, the most oxidised variety (Figure 3). Note that the ascorbate monoanion is more commonly described with the generic descriptor 'ascorbate'. Its monoanion structure enables combination with ions to create the various stable mineral salts of ascorbate, such as sodium ascorbate, calcium ascorbate and potassium ascorbate (Rose and Bode 1993).

This process can also be reversed, with ascorbate radicals converted back to ascorbate by monodehydroascorbate reductase at the expense of $\mathrm{NADH}$, and dehydroascorbic acid converted back into ascorbate by dehydroascorbate reductase at the expense of glutathione (for a detailed review see Noctor and Foyer 1998). This recycling in the ascorbateglutathione cycle is essential to mitigate ascorbate and glutathione consumption during their anti-oxidant function. 


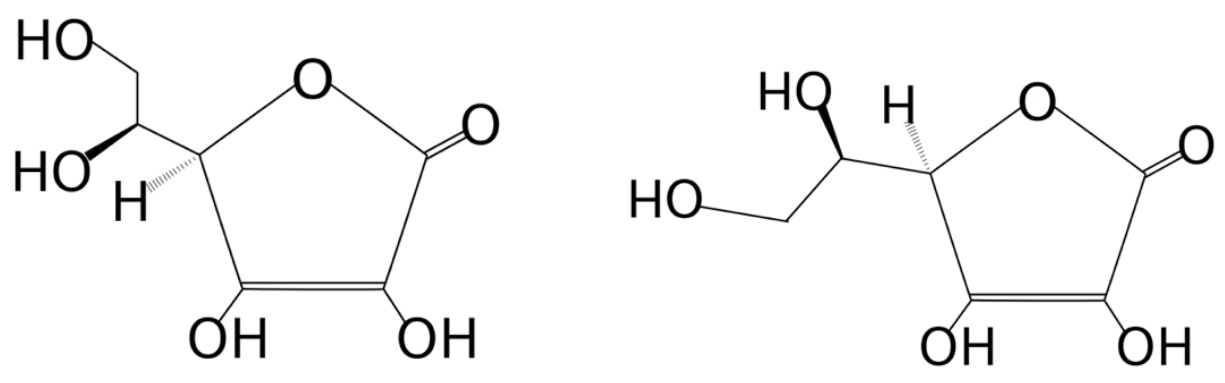

Figure 2 Stereochemical isomers L-Ascorbic Acid (L), D-Ascorbic Acid (R) 


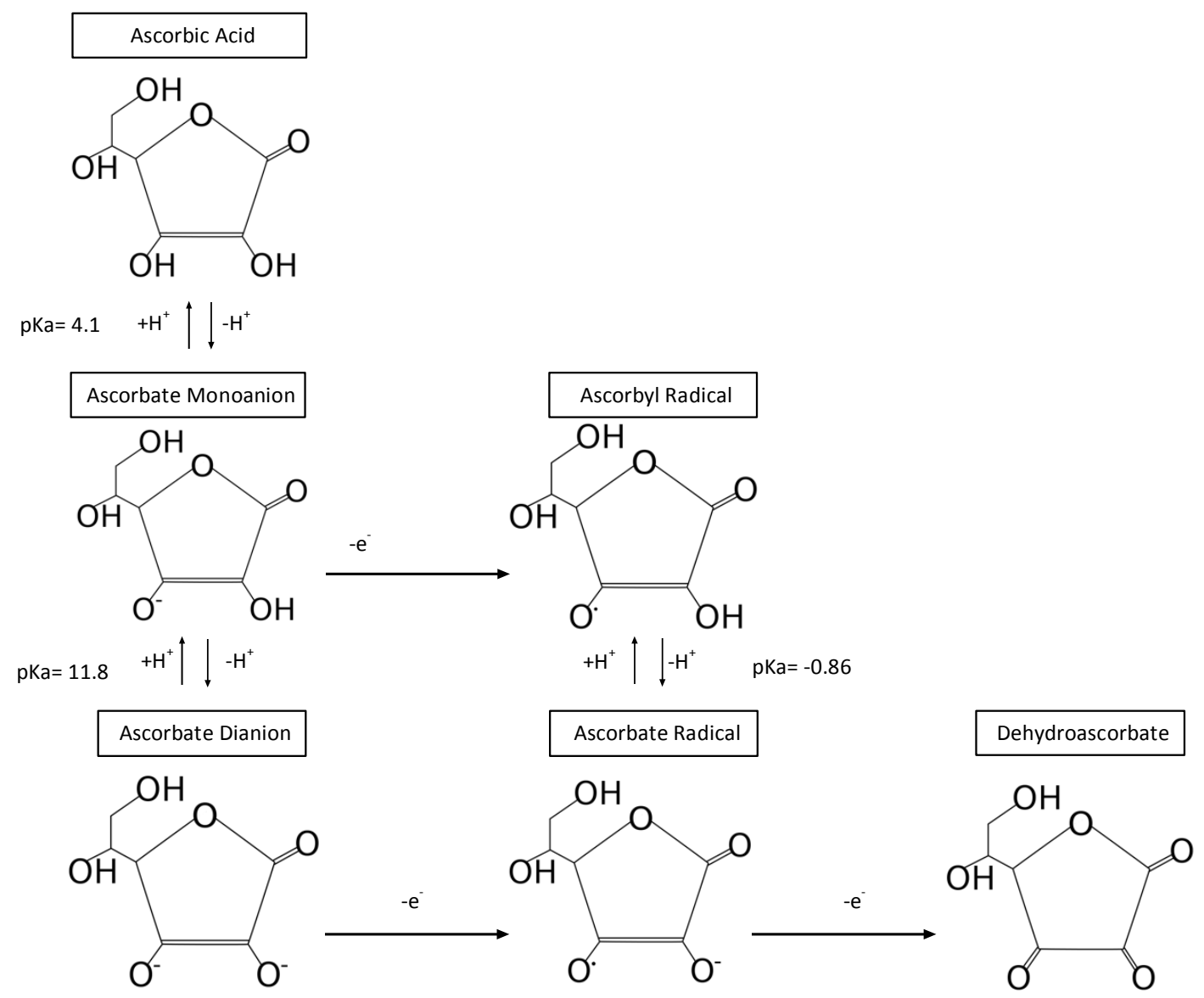

Figure 3. The Redox forms of Ascorbate

Adapted from (Domitrović 2006) 


\section{Ascorbate as an Antioxidant}

One of ascorbate's main roles is an effective antioxidant. In every cell, a finely tuned balance exists between free radicals (FRs) and reactive oxygen species (ROS) on one side, and antioxidants such as ascorbate, which counteract the harm they cause.

Free radicals are molecules containing ions that have a single unpaired electron in the outer valence shell. This property makes them highly reactive. Reactive oxygen species are also highly reactive compounds that contain oxygen. What they have in common is a short life, and high reactivity with nearby molecules in order to attain stability. This reactivity results in other molecules becoming electronically charged, and a chain reaction of oxidative injury is passed on (Sonntag 2006).

It should be noted the terms free radical and reactive oxygen species, although often used interchangeably, are not equivalent (Lushchak 2014). Many species fall into both categories, such as the hydroxyl radical, $\mathrm{OH}^{\circ}$. However, some molecules can only be accurately labelled with one term, for example hydrogen peroxide $\left(\mathrm{H}_{2} \mathrm{O}_{2}\right)$ contains oxygen but no radical.

Both FRs and ROS are a natural product of biology and not merely harmful. Reactive oxygen species are produced from normal metabolising mitochondria (complexes I and II), and neutrophils use them to kill pathogens. They can also regulate gene expression (Palmer and Paulson 1997; Sen and Packer 1996), relay intracellular signals (Colavitti, Pani et al. 2002; Fatma, Kubo et al. 2005), and activate important factors such as hypoxia-inducible factor 1 (HIF-1) and vascular endothelial growth factor (VEGF) (Liu, Hu et al. 2006; Xia, Meng et al. 2007).

However, an increase in the normal number of FRs or ROS causes oxidative stress, when the abilities of the antioxidant defence system are outweighed by the destructive oxidants (Varjovi, Valizadeh et al. 2015). 
During this imbalance, important molecules such as lipids, proteins and DNA are harmed. Depending on the extent and type of stress, this state can be very destructive, and has been linked to pathologies such as atherosclerosis, as well as more general processes such as inflammation, neurodegeneration, ageing and carcinogenesis (Emerit, Edeas et al. 2004).

Antioxidants can be either enzymatic such as superoxide dismutase (SOD) or catalase, or non-enzymatic and consumed, such as ascorbate. Non-enzymatic antioxidants are capable of neutralising free radicals because they are easily able to forfeit an electron to balance radicals, while not themselves becoming reactive, although some antioxidants transiently become radicals which are then regenerated by other antioxidants (for example vitamin $\mathrm{E}$ (a-tocopherol) is regenerated by vitamin $\mathrm{C}$ (Cooke, Evans et al. 2003). This process minimises damage and keeps radicals in check.

Ascorbate is well known to act as a potent scavenger of FRs and ROS (Dutta, Gautam et al. 2015), and by the same mechanism, reduce metal ions (Englard and Seifter 1986). Along with glutathione, ascorbate is arguably the most significant non-enzymatic antioxidant in a physiological context. Its structure allows easy contribution of either one or two electrons to radical compounds, while not becoming reactive itself (Bielski, Richter et al. 1975) by resonance stabilisation of the unpaired electron in the ascorbyl radical (Figure 4), or dismutation to ascorbate or DHA (Buettner and Jurkiewicz 1996). Further, DHA is recycled in the ascorbateglutathione cycle to regenerate the original antioxidant. 


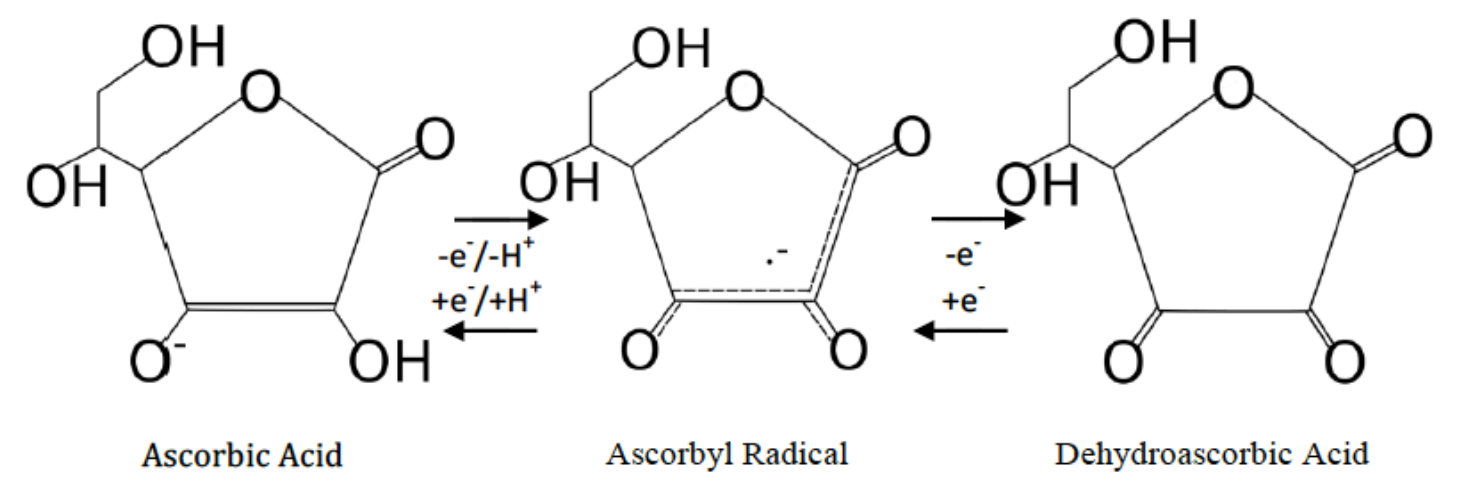

Figure 4. Resonance Stabilisation of the Ascorbyl Radical

Adapted from (Carr and Frei 1999) 


\section{Ascorbate as a Co-factor}

Another function of ascorbate is as a co-factor in various reactions. Ascorbate acts as a co-factor for a large and diverse family of enzymes known as the $\mathrm{Fe}$ and 2-oxoglutarate dependent dioxygenases, enzymes that also rely on co-substrate 2-oxoglutarate and a catalytic iron site to bring about hydroxylation of their respective substrate (Kuiper and Vissers 2014; Meredith and May 2013; Rebouche 1991). Three subgroups of these enzymes with relevance to cancer are the enzymes that hydroxylate collagen, HIF1, and 5-methylcytosine.

\section{Collagen}

The best known co-factor role for ascorbate is participating in collagen synthesis and prevention of scurvy. Scurvy only occurs in animals unable to produce the active enzyme L-gulonolactone oxidase that have insufficient ascorbate intake. Lack of ascorbate leads to incomplete hydroxylation of collagen, thus dysfunctional synthesis of the molecule, and symptoms such as swollen gums and poor wound healing. If the deficiency is not corrected, the eventual outcome is death. Most animals, excluding primates, guinea pigs, and fruit bats, are resistant to this disease, as they are able to undergo de novo ascorbate synthesis in the liver (Carpenter 1988).

In what has been called one of the first examples of a controlled trial, in the $18^{\text {th }}$ century British naval surgeon James Lind rediscovered and popularised the solution that foods high in vitamin $\mathrm{C}$, such as citrus, had antiscorbutic properties. Since Albert Szent-Gyorgyi successfully isolated the ascorbic acid molecule in 1928 (Carpenter 1988), science has learnt the mechanism by which ascorbate has this effect. Specifically, ascorbate acts as an essential co-factor of lysyl and prolyl hydroxylase, two important enzymes that catalyse the hydroxylation of their respective amino acids in collagen biosynthesis (Figure 5). Without ascorbate, hydroxylation of the residues, and therefore the correct structure of the collagen chains cannot occur, resulting in a weakened final molecule (Myllylä, Kuutti-Savolainen et al. 1978). 
The biosynthesis of collagen is dose dependent on the concentration of ascorbate available, with each molecule of ascorbate corresponding to addition of one hydroxyl to the collagen. This reaction oxidises the ascorbate molecule to DHA, and occurs at an early stage in collagen synthesis, in the rough endoplasmic reticulum (RER), before glycosylation (Vonk, Doulabi et al. 2010).

\section{HIF 1 - $a$}

Another enzyme ascorbate acts as a cofactor for is hypoxia inducible factor 1 (HIF-1) (Kuiper 2012). HIF1 is a transcription factor key to a cells response to hypoxia, or low oxygen conditions. Hypoxia is toxic to cells in general, yet it also provokes a plethora of responses that alter the way they function to promote survival, for instance changing from aerobic to anaerobic metabolism, glycolysis and transport of glucose, and encouraging factors such as vascular endothelial growth factor (VEGF) in order to increase angiogenesis and therefore blood supply and oxygen (Kuiper 2012; Ziello, Jovin et al. 2007).

HIF1 exists as a heterodimer consisting of HIF1- $\alpha$ and HIF1- $\beta$; both are expressed constitutively, although under normoxic conditions HIF1- $\alpha$ is rapidly degraded and the dimer cannot be formed. In this situation of normal oxygen, prolyl hydroxylase domain-containing proteins (PHDs) hydroxylate specific prolines in HIF1a's oxygen-dependent degradation domain, causing it to associate with the von Hippel Lindau (VHL) tumour suppressor protein. In turn, E3 ubiquitin protein ligase recognizes VHL and targets HIF-1 $\alpha$ for degradation. Another hydroxylase, factor inhibiting HIF1 (FIH-1), can block HIF1 from interacting with its target genes during normoxia by modifying a particular asparagine residue (Kuiper, Dachs et al. 2014).

Conversely, under hypoxic conditions, the process of degradation is prevented by inactivation of PHDs and FIH1, and HIF1a can accumulate. It translocates to the nucleus to dimerise with HIF1b and activate their many target genes. The targets of HIF1 include tumour relevant genes such as growth factors that encourage angiogenesis and cell proliferation (Kuiper 
and Vissers 2014), and genes that instigate the de-differentiation process epithelial mesenchymal transition (EMT) (Zhang, Huang et al. 2013).

In the context of cancer, hypoxia and HIF1 are very important. All solid tumours will have regions of hypoxia because of insufficient vascularisation. Hypoxia is also associated with resistance to irradiation and chemotherapy, and thus poor survival outcomes (Zhao, Quan et al. 2015). The HIF1 transcription factor promotes cell proliferation and survival, clearly fundamental processes to cancer development.

As ascorbate is a crucial cofactor for the activity of HIF1 hydroxylases, PHD and FIH1, ascorbate supplementation can inhibit HIF1, and so regulate its many downstream targets. Ascorbate can even counteract prevention of hydroxylase action by a number of hydroxylase inhibitors. This is thought to be principally via maintaining the iron domain of the two hydroxylases in the reduced state, and the effect is more pronounced for FIH1 than PHD (Kuiper, Dachs et al. 2014).

This has been borne out by experimental evidence. Firstly, the amount of ascorbate in human tumour tissue negatively correlates with HIF1 activation (Kuiper and Vissers 2014) as well as tumour phenotype and disease-free survival (Kuiper, Dachs et al. 2014). GULO mice that had ascorbate supplementation in their drinking water also displayed lower expression of HIF1 target genes, and decreased melanoma and lung cancer growth. (Campbell, Vissers et al. 2015). A xenograft study with mice that had been inoculated with cells that expressed a stable (i.e. constitutively active) form of HIF1 reported that ascorbate supplementation could not inhibit the tumour growth of these mutant HIF1 lymphoma, yet it could inhibit the activity of regular wild type HIF1 (Gao, Zhang et al. 2007).

Therefore, supplementation with ascorbate may be expected to lead to decrease of functional HIF1, and thus less hypoxia-dependent tumour cell proliferation and therapy resistance, and thus less hypoxia-dependent tumour cell proliferation and therapy resistance. 


\section{5-methylcytosine}

5 methylcytosine $(5 \mathrm{mc})$ is a DNA nucleotide, a cytosine, modified by a methyl group. Epigenetic modification of core DNA sequence by groups such as methyl residues is a large area of research, because these changes cause alterations in the way genes are expressed. As is the case with methylcytosines, which are involved in mammalian development and stem cell differentiation, among other functions (Tahiliani, Koh et al. 2009; Wu and Zhang 2011).

Ten eleven translocation (TET) methylcytosine deoxygenases (1-3) are the enzymes that catalase the addition of a hydroxyl group to $5 \mathrm{mc}$, modifying it to 5 hydroxymethylcytosine $(5 \mathrm{hmc})$. This is the first step in a process that ultimately demethylates the cytosine, altering any downstream epigenetic that would have otherwise occurred.

Interestingly, there may be a synergistic effect between HIF1 and TET. In tumorigenic neuroblastoma cells, hypoxia caused activation of HIF1, which in turn upregulated the expression of TET (Mariani, Vasanthakumar et al. 2014).

TET enzymes and their $5 \mathrm{mc}$ substrate also have relevance to cancer development. Abnormal methylation in general is strongly associated with cancer. Further, there is a marked lack of hydroxylated $5 \mathrm{mc}$ in cancerous cells relative to regular cells, although it is not yet clear whether this is symptomatic or causal (Ficz, Branco et al. 2011). Thus, enzymatic methylation modifiers such as TET have been implicated in oncogenic development (Wu and Zhang 2011).

It has been shown that ascorbate plays a co-factor role in TET activity (Minor, Court et al. 2013). By acting as an essential co-factor for TET, again thought to be primarily by maintaining the catalytic iron in a reduced state, it enhances the creation of $5 \mathrm{hmC}$ and alters its epigenetic action. For example, mouse fibroblasts cultured in ascorbate free medium have far less $5 \mathrm{hmc}$ than usually, but when ascorbate is added, levels of 
$5 \mathrm{hmc}$ increase in a dose dependent manner. This is dependent on the presence of functioning ascorbate transporters, and does not occur with other antioxidants. The mechanism is mediated by TET, as when TET is blocked with shRNA, the action of ascorbate is obstructed (Dickson, Gustafson et al. 2013).

In summary, ascorbate has been shown to function as a co-factor for a range of enzymes, and it is likely more interactions will be uncovered in the future. These connections have wide ranging consequences, not all of which have yet been determined.

However, ascorbate has been postulated to function in yet another way that has consequences for cancer therapy; as a prooxidant. 

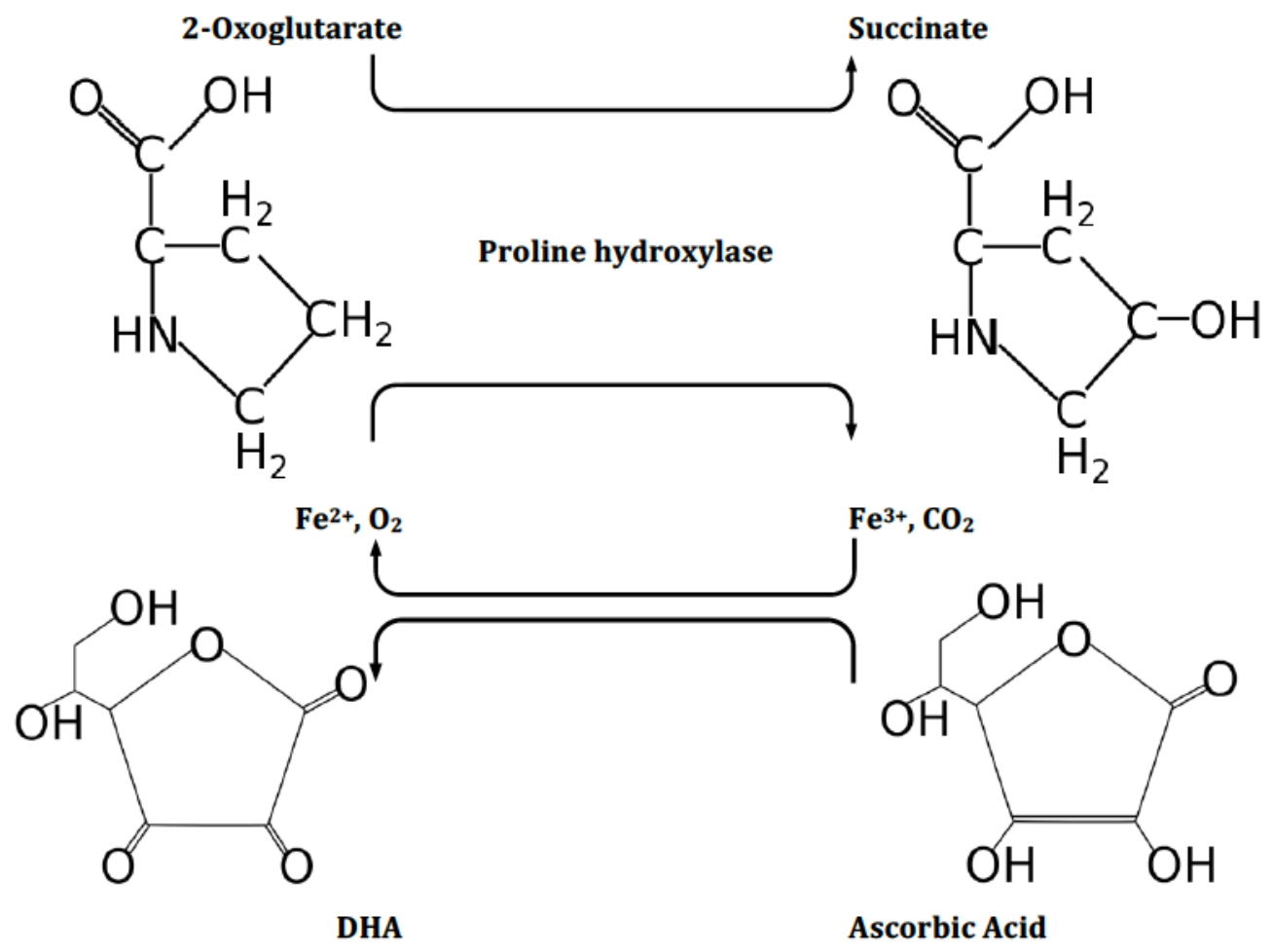

Figure 5. Ascorbate acts as a co-factor in the hydroxylation of proline in the biosynthesis of collagen

Adapted from (Division of Life Sciences 2011) 


\section{Ascorbate as a Prooxidant: The Fenton and Haber-Weiss reaction}

Ascorbate's role as a pro-oxidant was discovered relatively recently, compared to its ability to act as an antioxidant or co-factor. As such, less is known about the details and caveats to its action. But there is biological basis to suspect ascorbate is capable of exhibiting pro-oxidant properties. In high doses ascorbate could help overwhelm cancer cells and improve cancer outcomes. The proposed mechanism is the Fenton and Haber-Weiss reactions.

The Fenton reaction describes the general process of a reduced metal and an oxidant becoming an oxidised metal and an even stronger oxidant (Wardman and Candeias 1996). In his original paper to Chemical Letters, Henry John Horstmon Fenton described the formation of a violet coloured product (dihydroxymaleic acid) from tartaric acid (reductant), hydrogen peroxide (oxidant), and ferrous sulphate (catalytic metal source). The metal and oxidant used to explain the equation are iron and hydrogen peroxide, although other transition metals and oxidants can undergo the process. Fenton never investigated the mechanism behind the reaction that was named for him, he only used it as a tool in reactions.

The Haber-Weiss reaction built on Fenton's work, and was published in the 1930s. It provided a mechanism for creation of hydroxyl radicals from hydrogen peroxide and the superoxide ion (Haber and Weiss 1934). This reaction happens naturally, albeit slowly, in cells, yet the presence of transition metals can catalyse it and thus increase the rate. This Haber-Weiss reaction makes use of Fenton chemistry in Eq3.

$$
\mathrm{O}^{-}+\mathrm{H}^{+}+\mathrm{H}_{2} \mathrm{O}_{2} \longrightarrow \mathrm{O}_{2}+\mathrm{H}_{2} \mathrm{O}+\mathrm{OH} \quad \mathrm{Eq} 1 \text { (Net Haber-Weiss) }
$$

$\mathrm{O}^{-\cdot}+\mathrm{Fe}^{3+} \longrightarrow \mathrm{Fe}^{2+}+\mathrm{O}_{2} \quad \mathrm{Eq} 2$

$\mathrm{Fe}^{2+}+\mathrm{H}_{2} \mathrm{O}_{2} \longrightarrow \mathrm{Fe}^{3+}+{ }^{-} \mathrm{OH}+\cdot \mathrm{OH} \quad$ Eq 3 (Fenton Reaction) 
Hydrogen peroxide for the Fenton reaction can be from a range of sources, such as the dismutation of superoxide by SOD, or leakage from respiration in the mitochondria.

The significance of the formula took some time to be recognised (Wardman and Candeias 1996). At the time, it was thought that free radicals could not occur in vivo. And because the net Haber-Weiss reaction is thermodynamically unfavourable it needs a catalyst to have large effects. Eventually the role of transition metals in fulfilling the catalysis role, and the significant biological action potential was recognised. This reaction is commonly known as the iron or metal catalysed Haber-Weiss reaction, or the superoxide driven Fenton reaction.

Although the most common representation of the Fenton and HaberWeiss reaction, the formulas above are not the only example of this type of reaction. For example, the Fenton reaction can easily proceed with nitric oxide (NO) or a superoxide ion in the place of a transition metal to produce hydroxyl radicals.

Ascorbic acid can stand in for the superoxide ion in Part 1 of the Haber-Weiss reaction. This still achieves the redox active transition metal in Eq 5 required for Fenton chemistry in Eq 6:

$$
\begin{array}{r}
\mathrm{AA}+\mathrm{H}^{+}+\mathrm{H}_{2} \mathrm{O}_{2} \longrightarrow \mathrm{AA}+\mathrm{H}_{2} \mathrm{O}+\mathrm{OH} \quad \mathrm{Eq} 4 \text { (Net Haber- } \\
\text { Weiss) }
\end{array}
$$

$$
\begin{array}{ll}
\mathrm{AA}+\mathrm{Fe}^{3+} \longrightarrow \mathrm{Fe}^{2+}+\mathrm{AA}+\mathrm{H}^{+} & \mathrm{Eq} 5 \\
\mathrm{Fe}^{2+}+\mathrm{H}_{2} \mathrm{O}_{2} \longrightarrow \mathrm{Fe}^{3+}+{ }^{-} \mathrm{OH}+\mathrm{OH} & \text { Eq } 6 \text { (Fenton Reaction) }
\end{array}
$$

Another alternative is that the reduced metal produced in HaberWeiss Part 1 goes on to react with molecular oxygen and create hydrogen 
peroxide. This was the model proposed by Chen et al. (2008) in order to explain how high doses of ascorbate could act as a prodrug, and deliver hydrogen peroxide to the extracellular fluid, rather than the blood where the relatively higher concentrations of catalase would degrade hydrogen peroxide rapidly:

$\mathrm{Fe}^{2+}+2 \mathrm{O}_{2} \longrightarrow \mathrm{Fe}^{3+} 2 \mathrm{O}_{2}^{-\cdot} \quad \mathrm{Eq} 7$

$2 \mathrm{O}_{2}^{-\cdot}+2 \mathrm{H}^{+} \longrightarrow \mathrm{O}_{2}+\mathrm{H}_{2} \mathrm{O}_{2} \quad \mathrm{Eq} 8$

All of these reactions feed into each other, for example hydrogen peroxide can then go on to react with another reduced transition metal as in Eq 3, and create a hydroxyl radical (Parrow, Leshin et al. 2013).

These equations that show ascorbate's potential to create ROS is significant Hydrogen peroxide has a range of deleterious effects, being able to attack DNA, lipids in membranes, glucose metabolism and proteins. Yet hydroxyl radicals, created from the superoxide molecule in Eq 3, and from ascorbate in Eq 6, are even more deleterious to cell components when not detoxed by antioxidants. The hydroxyl radical is considered the most aggressive ROS, as it is the most reactive (Nappi and Vass 2000). It can be reduced and still be considered a ROS $\left(\mathrm{H}_{2} \mathrm{O}_{2}\right)$. However, the hydroxyl radical has a very short half-life, a nanosecond compared to on the order of minutes for hydrogen peroxide (Sonntag 2006), and so can only influence its immediate vicinity.

These reactive oxygen species produced by ascorbate, as with irradiation, produce a variety of types of DNA damage.

\section{Ascorbate Transport}

Although humans often consume a moderate amount of ascorbate from various sources in their diet, characteristics of the transporters that facilitate uptake contribute to relatively low serum levels, at least compared with other, ascorbate synthesizing species. 
Normally, ascorbate enters the human body through diet, and thus through epithelial cells of the small intestine. It then enters the circulatory system by diffusing through capillaries. Once in the kidney, ascorbate is transferred via the glomerulus capillary bed to the Bowmans capsule and from there taken up into renal epithelial cells. The body retains only what is reabsorbed in this manner, and excess proportions will be excreted in the urine (Ball 2008). This naturally limits the potential ascorbate serum concentration attainable.

Ascorbate is polar and relatively large, meaning it cannot readily diffuse across cell membranes and must be actively transported into cells, or transferred through facilitated diffusion. Ascorbate is transported via substrate transporters, either sodium dependent vitamin c transporters (SVCT1 and 2), or glucose transporter (GLUT) isoforms, depending on the redox form it takes (either ascorbic acid or DHA), and what tissue it is in (Rumsey, Kwon et al. 1997). Expression of these transporters varies by tissue type (Table 2). SVCT transporters utilize active transport in concert with sodium, while GLUT transporters employ facilitated diffusion.

SVCT 1 and 2 have high specificity for ascorbic acid compared with a very low affinity for DHA, and so will effectively only transport ascorbate in the ascorbic acid form. In contrast, GLUT transporters will only transport DHA, in addition to their traditional role as glucose transporters (Rivas, Zuniga et al. 2008; Rumsey, Kwon et al. 1997; Vera, Rivas et al. 1993) 
Table 2. Ascorbate Transporters

\begin{tabular}{|c|c|c|c|c|c|}
\hline $\begin{array}{l}\text { Sodium } \\
\text { dependent } \\
\text { active } \\
\text { transport }\end{array}$ & SVCT1 & $\begin{array}{l}\text { Found in } \\
\text { epithelial } \\
\text { tissue }\end{array}$ & $\begin{array}{l}\text { Transports } \\
\text { ascorbic } \\
\text { acid with } \\
\text { higher } \\
\text { capacity }\end{array}$ & $\begin{aligned} & \text { Role key for } \\
& \text { - } \text { Intestinal } \\
& \text { absorption } \\
& \text { - } \text { renal } \\
& \text { reabsorption } \\
& \text { - } \text { liver } \\
& \text { accumulation. }\end{aligned}$ & $\begin{array}{l}\text { KO mice = } \\
\text { excessive } \\
\text { urinary } \\
\text { excretion of } \\
\text { AA }\end{array}$ \\
\hline $\begin{array}{l}\text { Sodium } \\
\text { dependent } \\
\text { active } \\
\text { transport }\end{array}$ & SVCT2 & $\begin{array}{l}\text { Found in } \\
\text { brain (low } \\
\text { levels } \\
\text { elsewhere) }\end{array}$ & $\begin{array}{l}\text { Transports } \\
\text { ascorbic } \\
\text { acid with } \\
\text { higher } \\
\text { affinity }\end{array}$ & $\begin{array}{l}\text { Main transporter in: } \\
\text { - } \quad \text { Brain } \\
\text { - } \quad \text { Pituitary } \\
\text { - } \quad \text { Adrenals } \\
\text { Pancreas }\end{array}$ & $\begin{array}{l}\text { KO mice die } \\
\text { immediately } \\
\text { after birth of } \\
\text { brain } \\
\text { haemorrhage } \\
\text { and } \\
\text { respiratory } \\
\text { failure. }\end{array}$ \\
\hline \multicolumn{6}{|c|}{ SVCT1 and 2 have individual functionality, yet high sequence homology. } \\
\hline $\begin{array}{l}\text { Sodium } \\
\text { independent } \\
\text { facilitated } \\
\text { diffusion }\end{array}$ & $\begin{array}{l}\text { GLUT- } \\
1 / 2 / 3 / 4\end{array}$ & $\begin{array}{l}\text { Found } \\
\text { throughout } \\
\text { the body in } \\
\text { most cells }\end{array}$ & $\begin{array}{l}\text { Transports } \\
\text { DHA and } \\
\text { glucose (a } \\
\text { hexose } \\
\text { transporter) }\end{array}$ & $\begin{aligned} & \text { GLUT1 } \\
& \text { - } \text { Endothelial } \\
& \text { tissue } \\
& \text { - } \text { Blood brain } \\
& \text { barrier } \\
& \text { GLUT3 } \\
& \bullet \text { Neuronal } \\
& \text { Both } \\
& \text { - Osteoblast } \\
& \text { - Muscle } \\
& \text { - } \text { Retinal }\end{aligned}$ & $\begin{array}{l}\begin{array}{l}\text { KO causes } \\
\text { many } \\
\text { systemic } \\
\text { abnormalities: }\end{array} \\
\text { Growth, } \\
\text { adipose, } \\
\text { cardiac, liver, } \\
\text { gut issues } \\
\text { (e.g. Faconi- } \\
\text { Bickel, De } \\
\text { Vivo } \\
\text { diseases). }\end{array}$ \\
\hline \multicolumn{6}{|c|}{$\begin{array}{l}\text { SVCT info from (Tsukaguchi, Tokui et al. 1999); GLUT info from (Olson and Pessin } \\
\text { 1996; Pascual, Wang et al. 2004) }\end{array}$} \\
\hline
\end{tabular}

Although GLUT transporters carry ascorbate like SVCT transporters, they predominantly deal with glucose transport. This is because of the higher incidence of glucose than DHA, and the resulting competitive inhibition of DHA uptake. SVCT transporters are also more influential due to greater affinity for ascorbic acid than GLUT for DHA, as well as the large ascorbic acid to DHA ratio present throughout the body (Li, Chen et al. 2011). The severe ascorbate deficiency effects caused by knockout of SVCT transporters is evidence of their importance over GLUT transporters, especially for specific organs such as the brain. (Parrow, Leshin et al. 2013) However, in cells where only GLUT transporters are 
expressed, cells have no choice but to rely on them for their ascorbate needs (Agus, Gambhir et al. 1997).

In the CNS, astrocytes and neurons collaborate utilising both types of transporters to maintain ascorbate and thus redox homeostasis. This is despite ascorbic acid not being able to pass through the blood brain barrier and instead requiring conversion to DHA to allow transport before being reduced back (Agus, Gambhir et al. 1997). Under conditions of oxidative stress, DHA accumulates, as ascorbic acid is oxidised during detoxification of ROS. Astrocytes lack SVCT so cannot uptake AA like neurons. However, astrocytes contribute by uptake of DHA via GLUT1, reduction to ascorbic acid via glutathione (which they possess in relative abundance), and release for neurons to uptake. Reduction of dehydroascorbic acid can only happen inside cells (in endoplasmic reticulum). Neurons also express GLUT transporters so could carry out reduction of DHA themselves, however their antioxidant capacity is less than that for astrocytes (GarciaKrauss, Ferrada et al. 2015).

Transport of ascorbate specifically to tumour tissue has been investigated. It is well known that cancer patients have much reduced systemic ascorbate concentrations (World Cancer Research Fund 2007). Yet this does not necessarily translate into tumour tissue, for instance, brain tumours have less ascorbate, but other tumours such as breast and lung have been shown to have more ascorbate. It is not yet known ascorbate scarcity in tumours is owing to insufficient vascularisation, or alterations in the expression of SVCT transporters. Intriguingly, the ascorbate concentration of tumour tissue has been shown to correlate with disease free survival in some tumours (Kuiper, Dachs et al. 2014).

\section{Ascorbate Pharmacokinetics}

One of the reasons ascorbate was neglected for so long as a possible anti-cancer therapeutic is is because the vast difference the mode of application makes, specifically oral or intravenous, was not appreciated (Padayatty, Sun et al. 2004). 
The SVCT2 transporters are easily saturatable. The Km value range, depending on cell type and $\mathrm{pH}$ is $65-237 \mu \mathrm{M}$ for SVCT1 and 8-62 $\mu \mathrm{M}$ for SVCT2 (Savini, Rossi et al. 2008). This indicates higher concentrations will not be taken up by the SVCT transporters. This particularly applies to the intestines during oral delivery.

The total body pool of ascorbate is generally 1.5-2 grams, yet the levels of ascorbate vary throughout the body (Kallner, Hartmann et al. 1979). Ordinarily, the brain, especially the pituitary gland, and the adrenal medulla have the highest levels (Patak, Willenberg et al. 2004).

The ascorbate serum concentration can range from $\sim 6$ to $92 \mu \mathrm{M}$, depending on an individual's typical intake (Levine, Conry-Cantilena et al. 1996). A recent pharmacokinetic model predicted normal tissue under average serum ascorbate concentrations would produce extracellular concentrations of around 20 to $50 \mu \mathrm{M}$, and intracellular concentrations of between around 300 and $700 \mu \mathrm{M}$ (Kuiper, Vissers et al. 2014). Some specific cells may contain higher concentrations, such as neutrophils and leukocytes, which have up to 30 times greater ascorbate than a typical cell, in order to protect themselves from the ROS they use to destroy pathogens (Wang, Russo et al. 1997).

One hundred milligrams of ascorbate orally daily are enough to saturate the body in healthy individuals, as indicated by leukocyte concentrations and the appearance of urinary excretion. This amount is enough to generate a fasting plasma concentration of approximately $60 \mu \mathrm{M}$, yet increasing the dosage by a factor of 10 to $1000 \mathrm{mg}$ daily can only produce $75-80 \mu \mathrm{M}$. Oral doses even greater than this cannot generally increase plasma concentration further (Levine, Conry-Cantilena et al. 1996; Padayatty and Levine 2001; Padayatty, Sun et al. 2004). The higher end of this plasma concentration scale, around $80 \mu \mathrm{M}$, has been reported as ideal to reach tissue saturation (Kuiper, Vissers et al. 2014). 
Ascorbate concentration in plasma follows a sigmoid function (Levine, Conry-Cantilena et al. 1996; Newton, Morgan et al. 1983). Newton et al. found that plasma concentrations plateau at the renal threshold of $80 \mu \mathrm{M}$. Levine et al. demonstrated that the steep portion of the concentration curve came about with doses between 30 and $100 \mathrm{mg}$ and that complete saturation transpired at 1,000 mg daily. For comparison, the current New Zealand recommended dietary intake for vitamin $\mathrm{C}$ ranges from 45 to $85 \mathrm{mg} /$ day, with a "prudent limit" of 100mg/day (Australian Government Department of Health and Ageing National Health and Medical Research Council and New Zealand Ministry of Health 2006).

Physiological half-life of ascorbate is dependent on the blood concentration and whether ascorbate is plentiful. In periods of deficiency, renal transporters actively uptake ascorbate to prevent excretion. In this situation, at levels lower than around $85 \mu \mathrm{M}$, half-life is inversely proportional to concentration, while only above $85 \mu \mathrm{M}$ does half-life become constant, on average 14.2 days. In contrast, when ascorbate intake is high and concentration spikes, excretion is swiftly increased and half-life is greatly decreased to in the region of 30 minutes (Levine, Padayatty et al. 2011).

An additional limit to ascorbate intake is that transporters are down regulated in response to ascorbate itself. This negative feedback occurs at the level of translation and decreases uptake when consumption increases. Above 1g/day intake can drop to lower than $50 \%$ of even normal absorption (Kallner, Hartmann et al. 1979). These characteristics significantly limit the amount of ascorbate that can be derived orally.

Absorption is further altered by portioning of ascorbate quantities. There is evidence that fractionating dosages can increase the absorption such that smaller individual doses given several times per day can increase serum concentration more than one larger dose daily. (Australian Government Department of Health and Ageing National Health and Medical Research Council and New Zealand Ministry of Health 2006) 
However, these limitations are overcome when ascorbate is applied intravenously. 0.1 to 1.0 grams per kilogram of body mass gives peak concentrations over $10 \mathrm{mM}$, and Riordan et al. detailed how an infusion of up to $115 \mathrm{~g}$ over 8 hours in patients produced prolonged plasma concentrations of up to 5mM (Mikirova, Casciari et al. 2013). A single infusion intravenously of $1.25 \mathrm{~g}$ gives plasma concentration $\sim 7$ times higher than could be achieved with that same dose orally. Pharmacokinetic modelling has also attempted to predict potential plasma concentrations achievable with ever greater intravenous infusions. For example, it is predicted that $100 \mathrm{~g}$ intravenous infusion would transiently reach a concentration of nearly $20 \mathrm{mM}$, dwarfing the micromolar concentration that comes from the maximally tolerated oral dose of 18 grams per day (Padayatty, Sun et al. 2004).

This differentiation in ascorbate concentration is key. Intravenous delivery results in much higher systemic ascorbate concentrations, and it is only these high concentrations that can exhibit pro-oxidant effects capable of destroying cancer cells.

In short, there are multiple examples of ascorbate causing DNA damage, especially when in culture with transition metals (Halliwell and Gutteridge 1986). There is also substantial evidence of the ability of intravenous application of ascorbate in vivo to reach the concentration capable of inducing pro-oxidant effects in vitro. Yet, whether this translates to effective cytotoxicity of cancerous cells, and, more importantly, selective therapeutic benefit in vivo is far from settled.

\section{Ascorbate as Disease Therapy}

While ascorbate definitively prevents scurvy, there is controversy over the effectiveness of the antioxidant role of ascorbate in preventing diseases other than scurvy. For example, Linus Pauling, the only person to win two unshared Nobel Prizes, suggested that large doses of ascorbate are capable of preventing or significantly reducing the symptoms and duration 
of the common cold. Subsequent studies found some positive effects, although a recent Cochrane meta-analysis concluded that, "The lack of effect of regularly administered vitamin $\mathrm{C}$ on the incidence of the common cold in the general population throws doubt on the usefulness of this practice [...] It does not seem worthwhile to carry out further regular supplementation trials in the general population." However, the writers did allow that the small positive effects seen, combined with the low cost of regular ascorbate supplementation, indicated that it may be worthwhile for cold-prone and especially athletic individuals to experiment with ascorbate supplementation of 1-2 grams/day (Hemila and Chalker 2013).

The potential mechanism of action proposed to explain the mild benefit of ascorbate against the common cold is as an immune stimulator. Ascorbate both enhances T-cell proliferation, a key white blood cell member of the immune system, and decreases apoptosis (Naidu 2003).

Other studies have investigated ascorbate's effectiveness in preventing atherosclerosis and cardiovascular disease. The ARIC (Atherosclerosis Risk in Communities) study reported subjects older than 55 had a statistically significant inverse relationship between Vitamin C intake and average carotid intima media thickness, an indicator of atherosclerosis progression. While analysis of epidemiological data relating to heart health found that higher vitamin $\mathrm{C} /$ vitamin $\mathrm{E}$ ratio correlates with lower risk of CVD (Gey 1998).

\section{Ascorbate as Cancer Therapy}

There is epidemiologic evidence of the positive preventative anticancer effects of antioxidant ascorbate. Development of cancer, especially age-related, is caused in part by oxidative damage to DNA. There is overwhelming support that a diet high in fruits and vegetables decreases the risk of cancer (Lunet, Valbuena et al. 2007; World Cancer Research Fund 2007), and there are some studies that show this may be specifically due to the ascorbate content (Halliwell 2001; Loria, Klag et al. 2000). Some populations whose consume large amounts of ascorbate have a decreased 
risk of some types of cancer (Gey 1998). For example, there is a correlation between eating foods high in ascorbate and lower risk for stomach cancer, cervical cancer, and oesophageal cancer (Block 1991; Bo, Lu et al. 2015), although results are not conclusive (Bjelakovic, Nikolova et al. 2004; World Cancer Research Fund 2007 ), and this does not appear to be generalizable to all forms of cancer (Gaziano, Glynn et al. 2009; Head 1998; Lin, Cook et al. 2009; Miura and Green 2015).

Despite its traditional role as an antioxidant, ascorbate has been investigated as a cancer therapy that is effective through pro-oxidant means. However, when scientists first formed the theory of ascorbate's anti-cancer effect, it was the role in collagen creation that was the proposed mechanism, as its prooxidant capacity had not been learned. Today, both functions are thought to contribute to potential effectiveness.

A US doctor, William J. McCormick, first developed the theory that ascorbate may potentially decrease the incidence of cancer in the $1950 \mathrm{~s}$. He thought cancer was an ascorbate deficiency disease and improving collagen with supplemental ascorbate could prevent it. His previous work claimed to show that very large doses of ascorbate had antibiotic effects against tuberculosis, scarlet fever and other infectious diseases when administered intravenously or intramuscularly (McCormick 1952).

McCormick synthesised several strands of information to produce his theory. In two papers that were published in Archives of Paediatrics (McCormick 1954; McCormick 1959), McCormick explained how he believed cancer could prevent and treat cancer at early stages. At the time, the extent of knowledge surrounding the cause of cancer was limited to the hypothesis that it was a combination of "chronic irritation" such as that caused by soot inhalation, coupled with a removal of restraints on cell growth (McCormick 1954). He noticed that cancer is characterised by extensive uncontrolled growth and metastases, and that at the time (as now), the medical community's focus was on medicinal and surgical 
treatments, rather than prevention or examining the impact of metabolism and diet.

By the time McCormick was formulating his theory, ascorbate's function as a collagen, and in fact general connective tissue reinforcer was well known. Furthermore, it was widespread knowledge that cancer patients had much lower ascorbate serum concentrations than healthy individuals, even accounting for their sub-par nutrition.

McCormick stressed how the basement membrane, which contains collagen, is a key boundary for metastasising cells, and if that boundary was to be breached, or ineffectually repaired, the potential for tumours and metastasis is greatly increased. Further, connective tissue is another barrier to metastasis, and because it too consists of collagen, it could be strengthened by ascorbate (McCormick 1954).

With this information regarding ascorbate's abilities to both reinforce connective tissue and inhibit the destruction of connective tissue, McCormick hypothesised that the primary cause of cancer development was a lack of ascorbate that in turn caused weakened connective tissue and allowed rogue cells to subvert their constraints. He was the first to propose the link between a lack of ascorbate and a predisposition to cancer. Although he didn't carry out any practical studies to demonstrate the validity of his idea, his theory would open the door to later scientists who attempted to uncover whether ascorbate did indeed improve cancer outcomes.

As well as strengthening the barriers to metastasis, ascorbate was found to inhibit the molecules that break down these barriers. Another component of the extracellular environment is hyaluronic acid, the simplest glycosaminoglycan in the extracellular matrix. Karl Meyer and John Palmer discovered hyaluronic acid isolated from the vitreous of bovine eyes in the 1930s (Selyanin, Boykov et al. 2015). 
Break-down of hyaluronic acid enables metastasis, and hyaluronidase is the enzyme cancer cells use to break down hyaluronic acid to do so. As early as the 1950s, Reppert, Donegan and Hines (Reppert, Donegan et al. 1951) showed that ascorbate could effectively inhibit hyaluronidase's catabolic effect on hyaluronic acid. More recently it has been reported that L-ascorbic acid, D-isoascorbic acid and DHA can all inhibit hyaluronidase (Okorukwu and Vercruysse 2003) and ascorbic acid derivatives can also obstruct the function of the enzyme, to a much greater degree than the parent compound. (Spickenreither, Braun et al. 2006)

Inspired by the theories that had been published, Scottish physicians Ewan Cameron and Allen Campbell produced one of the first trials of high dose ascorbate in 1974. This particular trial aimed to treat patients that had been judged 'untreatable' and had not received conventional therapy, although they had previously been treating many patients, including some that had traditional therapy in conjunction with high-dose ascorbate (Cameron, Campbell et al. 1975).

50 cancer patients took $10 \mathrm{~g}$ of ascorbate per day for the initial 10 days intravenously, and orally thereafter, while some took only oral ascorbate. The doses were fractionated into $2.5 \mathrm{~g}$ of ascorbate four times daily. Interestingly, Cameron and Campbell note in their report that they believe in hindsight that intravenous administration is "probably unnecessary", except in cases where for some reason the patient is unable to consume ascorbate orally (Cameron, Campbell et al. 1975).

Cameron and Campbell reported a large variety of responses within their 50 patients, ranging from no response, to growth retardation, to tumour necrosis. Of course due to the lack of controls in this particular study, no statistical inferences could be made, yet they claimed that a subjective evaluation clearly showed the patients survived "longer than reasonable clinical expectation." However, there were some promising measures regarding factors such as quality of life and erythrocyte 
sedimentation, which they compared with an "expected trend" (Cameron, Campbell et al. 1975).

Later that decade, Cameron collaborated with the prestigious Nobel Prize winner Linus Pauling on a larger scale trial (Cameron and Pauling 1979). As mentioned previously, Pauling had already been promoting the notion of high-doses of ascorbate as a therapy for the common cold (see 'Ascorbate as an Antioxidant').

A series of terminal cancer patients were treated in 1976. In this study, detailed in two papers published in the Proceedings of the National Academy of Sciences (PNAS), Cameron and Pauling set forth how 100 again 'untreatable' cancer patients (50 of whom were reported on previously) were given ascorbate and compared with 1,000 matched controls from the same hospital. Their protocol was based on the previous trial, with patients receiving both intravenous and orally administered ascorbate (Cameron and Pauling 1976).

Pauling and Cameron found longer survival times with ascorbate than "what would be expected". The mean survival time for ascorbatetreated patients was over 160 days longer than that of the matched controls, who only survived on average 50 days after being deemed untreatable. There was even decreased tumour growth and cases of tumour regression. In a large proportion of ascorbate treated patients, survival was determined to be 4 times greater than control patients, while in $10 \%$ of ascorbate treated patients their survival was even more significantly lengthened such that a number were still alive when the paper was published. Pauling and Cameron did not go so far as to proclaim this proportion 'cured', but estimated their survival to be approximately 20 times greater than the control group. The report measured survival only, and did not consider other parameters such as quality of life. The scientists were influenced by McCormick's investigations and proposed the increase in survival was due to enhanced collagen production and 'restrained' cancerous cells rather than outright killing (Cameron and Pauling 1976). 
However, there were significant problems with the Cameron and Pauling trials. For instance, there was no genuine control group.

Retrospective controls were used, such that patients treated with ascorbate were compared with past patients that, from their medical records, had apparently similar levels of disease. An independent reviewer carried out the matching, yet the trial could not be double-blinded or placebo controlled.

This, and other aspects of the trial brought understandable criticism (Creagan, Moertel et al. 1979). Some questioned the reliability of the decision to pronounce patients untreatable, as it was in Pauling's interest to include those that were less ill in the treatment arm, thus artificially lengthening the overall survival for this group. Whether the sample and control group were truly representative subpopulations was another doubt raised by detractors.

The pair's second trial attempted to correct some of this criticism. For instance, controls were re-selected, taking extra care to match as accurately as possible to the treatment group for cancer type, sex, and age. Furthermore, they carried out statistical tests to determine the time between date of admission to the hospital and date deemed 'untreatable'; if the control and treatment groups were matched accurately, these times should be similar for the two groups. Instead of diluting the apparent effectiveness of high-dose ascorbate, this updated analysis strengthened its ostensible potential. The ascorbate-treated patients had a mean survival time approximately 300 days greater than controls. Survival times longer than a year being deemed untreatable were observed for $22 \%$ of the ascorbatetreated patients compared with only $0.4 \%$ of the controls (Cameron and Pauling 1976).

Yet critics were not satisfied (Comroe 1978). The essence of the trials as non- blinded nor controlled by placebo left much to be desired; as well as the fact that only 10 new ascorbate treated patients had been 
replaced, and half of the controls had been drawn from the first study. Furthermore, Pauling and Cameron appeared to have a rather unscientific faith in the molecule's efficacy. In Cancer and Vitamin C, published in 1979, Cameron said that "he did not feel justified in carrying out a randomized, double-blind trial because he was convinced of the value of the vitamin for his patients, and it would therefore be unethical to withhold it from those who would be serving as controls." (Carpenter 1988)

In response to the controversy, the Mayo Clinic undertook two double blind, randomised, placebo-controlled studies involving patients with advanced cancer. The studies, reported in $1979(n=150)$ and 1985 $(\mathrm{n}=100)$, found that patients given $10 \mathrm{~g}$ of vitamin $\mathrm{C}$ daily did no better than those given a placebo.

In the first report only a small proportion of their subjects had not previously received therapy. There was no difference between the treated and control groups in age, sex, previous therapy, type of primary tumour, or ECOG (Eastern Cooperative Oncology Group) score. Survival rates between the two groups were so similar that the curves overlapped, and there were also no statistically significant differences in symptoms (Creagan, Moertel et al. 1979).

Their second paper attempted to contend Pauling's objection that the cause of the negative results in the first study was due to the patients' previous chemotherapy. The Mayo authors contested that if in fact ascorbate was incompatible with chemotherapy then there was no choice but to opt for the proven chemotherapy over an unproven vitamin (Richards 1988). Despite this argument, and to appease Pauling and his high-profile supporters, a second trial was performed (Richards 1988). They randomised 100 patients with advanced colorectal cancer, none of whom had received prior chemotherapy and 4 of whom had received radiation, into control and treatment groups. Again, patients were stratified by various parameters and again, no statistically significant differences were found between the two groups in survival and symptoms. It seemed that the theory of high-dose 
ascorbate in treating cancer had been invalidated (Moertel, Fleming et al. 1985).

In the face of this new evidence, the National Cancer Institute Panel determined that there was "insufficient evidence" for high-doses of ascorbate as a cancer therapy (Richards 1988). However, in both of these trials, ascorbate was only given orally, a fact not commented upon by the authors.

\section{Ascorbate as a Prooxidant Cancer Therapy}

Since investigation into the particular pharmacokinetic processes that regulate ascorbate, and the discovery of its capacity to act as a prooxidant, a multitude of studies have investigated the possibility of large doses of the molecule causing antitumor effects.

Despite the lack of conclusive evidence regarding its effectiveness, the use of high-dose ascorbate as a cancer therapy is increasing, prompted by the large number of orthomolecular healthcare practitioners who dispense it (Padayatty, Sun et al. 2010). These health providers predominantly sit at the periphery of mainstream science based medicine, but are well served by a clientele of patients interested in 'holistic health', including vitamin products (Richards 1988). A large advantage accentuated by the proponents of ascorbate as an anticancer treatment is its low toxicity. Ascorbate, even in very high doses, is generally well tolerated (Cameron, Pauling et al. 1979).

Yet overall, in the scientific literature, there seems to be a general consensus that ascorbate treatment alone has no significant effect on the development of tumours in vivo, (and therefore any possible effect would only be due to interaction with radiation or chemotherapy) (Kurbacher, Wagner et al. 1996). Even Pauling and Cameron intended for high-dose ascorbate to be used as an adjuvant therapy to increase the body's defences to cancer, while traditional therapy attacked it. 


\section{In Vitro Trials}

A wealth of trials exists on the effect of ascorbate on cancerous cells in vitro, whether as a monotherapy, or in combination with traditional therapies such as chemotherapy or radiation. The majority of studies that investigate ascorbate and cancer appraise pancreatic, ovarian, or breast cancer (Du, Martin et al. 2010; Kurbacher, Wagner et al. 1996; Ma, Chapman et al. 2014). Despite this extensive literature, and in consideration of the focus of this investigation and the unique environment of the brain, only in vitro studies that considered brain cells, and especially glioblastoma multiforme, will be overviewed.

Laurenzi et al. (1995) only reported cell death of SK-N-BE(2) neuroblastoma cells in response to either $10 \mathrm{~nm}-1 \mathrm{mM}$ of ascorbate, or 1$5 \mathrm{mM}$ of hydrogen peroxide (the mechanism by which ascorbate has its prooxidant effect, see 'Ascorbate as a Prooxidant'). They found $32 \%$ and $43 \%$ cell fatality in response to hydrogen peroxide and ascorbate respectively. Interestingly, this was much less than that observed with melanoma cells under the same conditions, where over $80 \%$ of cells died. The mechanism of this cytotoxicity was apoptosis, or programmed cell death.

A large set of cell lines were assessed by Chen et al. (2008) to confirm ascorbate's prooxidant effect and cytotoxicity. In all, 43 types of cancerous cells and 5 normal cell varieties were evaluated, including a murine neuroblastoma (2a), 3 kinds of rat GBM (C6, 9L, RG2), and nine human GBM cell lines. Survival, indicated by $\mathrm{EC}_{50}$ was measured and reported, indicating several interesting outcomes. As the authors expected, none of the non-cancerous cell lines displayed cytotoxicity in response to even the highest ascorbate dose of $20 \mathrm{mM}$. As for the cancer lines, there was a great variation in the cell survival, even amongst GBM only. For instance, the murine neuroblastoma, the rat GBM, and one of the human GBM lines (S635) had an $\mathrm{EC}_{50}$ of less than 1mM. The remaining GBM cell lines $\mathrm{EC}_{50}$ ranged from less than 5mM (LN229 and A172) to U383 and U87, which appeared impervious to even $20 \mathrm{mM}$ of ascorbate. A subsequent catalase 
test with a subgroup of the cell lines indicated that ascorbate cytotoxicity was mediated by hydrogen peroxide.

Thomas et al. (Thomas, Vezyraki et al. 2005) investigated the effect of ascorbate on various cancer cell lines in the context of cell cycle analysis. They published that although $2 \mathrm{mM}$ of ascorbate or DHA could induce significant death in HeLa (cervical adenocarcinoma) cells, concentrations up to $5 \mathrm{mM}$ had very little effect on the GBM cell line T98G. However, it could delay activation of Cdc2 and entry of cells into mitosis. This result was in contrast to Chen et al.'s findings in the previously mentioned study, where a 2-hour ascorbate treatment followed by a 24-48hour recovery period resulted in an $\mathrm{EC}_{50}$ of around $8 \mathrm{mM}$. A 48 -hour treatment in $5 \mathrm{mM}$, such as that used by Thomas et al, could be expected to have decreased viability at least somewhat, yet no concentration below $5 \mathrm{mM}$ decreased viability more than $20 \%$. This may indicate that at least for this particular GBM cell line, an effective concentration for cell death only arises at doses between $5 \mathrm{mM}$ and $8 \mathrm{mM}$, or alternatively, some particulars of protocol influenced the efficacy of ascorbate on this cell line between the two experiments.

The GBM cell line T98G were also studied by Naidu et al. (Naidu, Tang et al. 2001), who treated them with small concentrations (1-175 $\mu \mathrm{M})$ of a lipophilic ascorbate derivative, ascorbyl stearate, for 24 hours. This caused cells to accumulate at S/G2-M, inhibited clonogenicity and proliferation, and induced apoptosis. The authors postulated that these ascorbate effects were mediated either by creation of ascorbyl radicals, or by decrease in insulin-like growth factor I receptor (IGF-IR), because ascorbate dose and time dependently modulated the expression of IGF-I, an important regulator of the cell cycle. In this case, mechanism of action may not have been via prooxidant activity, but this was not determined by the authors, as no catalase assays or the like were carried out. The same authors also investigated the effect of ascorbyl stearate and ascorbyl palmitate on a murine glioma line, G-26, and again found that both ascorbate derivatives dose-dependently decreased proliferation and viability of cells. It is 
interesting to note the heightened anti-proliferation potency ascorbate derivatives compared with ascorbate alone, although they are less studied than their parent molecule and so safety is less assured, they are generally considered to be non-toxic (Elmore 2005).

In contrast, some studies have investigated the potential for ascorbate to act in concert with other therapies.

Allen et al. (2012) investigated the effect of radiation in combination with ascorbate on the U87 line, another GBM derived cell. 2$5 \mathrm{mM}$ of ascorbate in the cell media caused cytotoxicity and radiosensitization in U87 cells. The cytotoxicity and radio-sensitization was inhibited by catalase and by metal chelators, indicating the importance of metal catalysis and supporting hydrogen peroxide as the key mediator of oxidant activity. The effect was the same in non-small cell lung carcinoma cells.

In our own lab, a number of papers have outlined the prooxidant capacity of ascorbate in vitro (Castro, McConnell et al. 2014; Grasso, Fabre et al. 2014; Herst, Broadley et al. 2012). Human GBM cell lines and primary GBM cells, a human glial cell line, and primary human vascular endothelial cells as well as the murine equivalent of GBM, GL261 (see 'GL261 Mouse Model of GBM') and regular astrocytes (C8D1A) have been used. We have also investigated the effect of ascorbate damage in concert with the traditional therapy radiation.

Viability in response to treatments was measured via trypan blue exclusion. As expected, normal astrocytes were found more resistant to radiation than cancerous cells. Human cell lines and late passage primary cells were the next resistant, while early passage primary cells and the murine GBM cells were the least resistant to radiation relatively.

Ascorbate addition to radiation treatment increased its effectiveness. 1 Gy of irradiation did not decrease cell viability a great deal; cells were 85- 
$90 \%$ viable for primary cells and $75 \%$ in the most radiosensitive GL261 cells, yet a sub-lethal dose of ascorbate, only $0.5 \mathrm{mM}$, could decrease cell viability to $70-80 \%$ and $50 \%$ for primary cells and GL261 respectively. Similarly, the level of DSBs, as indicated in proxy by the appearance of IH2AX, appears synergistically increased by combination treatments as opposed to monotherapies.

Significantly, GBM primary cells, the most relevant in vitro representation of in situ tumours, underwent greater than additive decreases in viability when treated with both therapies, potentially signposting a synergistic effect for ascorbate and radiation treatment in vivo.

Herst et al. showed that although both $5 \mathrm{mM}$ of ascorbate and 6Gy of irradiation damaged primary GBM cells, combination treatment generated significantly more harm, and killed more cells than individual treatments. GBM cell lines were also radio-sensitised by addition of ascorbate in Castro et al., but although this effect was consistent among the cell lines, variability was high with regards to the degree each cell line could be radiosensitised. Cell lines also varied in response to individual treatments; in some cell lines, treatment with ascorbate caused more DSBs than irradiation.

When originally comparing murine astrocytoma and astrocytes, we found the normal cells were less affected by the addition of ascorbate than cancerous cells, a promising finding for potential therapy which indicated specificity for the treatment. However, in a more recent paper, normal untransformed glial cells fared no better than GBM cells in the effect of treatment on DNA damage, repair, viability, and clonogenicity. Both GBM cells and normal cells (SVGp12, a normal glial cell line and HUVEC, a vascular endothelial cell line) were harmed equally by the oxidative stress caused by radiation and/or ascorbate. 
The GBM, SVGp12 and HUVEC cell lines have additionally been investigated for their antioxidant capacity, in recognition of the probably prooxidant mechanism of ascorbate. Cell lines differed in their basal antioxidant ability. The degree of antioxidant in a given GBM cell line will influence the amount of ROS produced in that cell from a given dose of ascorbate, and, interestingly, has been reported to correlate with the ability of the cell to repair damage, as measured by the resolution of the $\mathrm{rH} 2 \mathrm{AX}$ signal.

The effectiveness of combination treatments in GBM primary cells was dose dependent on both ascorbate and irradiation. As measured by cell viability, sub-lethal ascorbate doses of $0.5 \mathrm{mM}$ increased the cytotoxic effect of irradiation 2-fold, yet when added at $5 \mathrm{mM}$, the effect was increased 4-fold. In a similar way, the cytotoxicity of 6Gy of irradiation could be increased 5-fold by addition of the smaller concentration, while a full $5 \mathrm{mM}$ increased cell death 10 -fold.

Cell cycle analysis was also investigated using DNA content analysis. It was determined that irradiation only resulted in G2/M arrest in GBM cells, but that addition of ascorbate could prevent this arrest. It was later discovered that ascorbate caused accumulation in $\mathrm{S}$ phase. This is significant therapeutically, because cell cycle arrest is a method of allowing DNA damage to be repaired, and thus limits the ability of irradiation to provoke damage induced cell death. If ascorbate can prevent the cell cycle blockade and DNA repair, it bodes well for ascorbate increasing the effectiveness of irradiation, complementary to the pro-oxidant effect.

The mechanism of ascorbate damage is certainly $\mathrm{H}_{2} \mathrm{O}_{2}$ mediated, for catalase mostly abrogated the effect of ascorbate, yet the precise pathway of cell death with these treatments is not yet determined. Herst et al. showed that the cytotoxic effect in GBM was not mediated by either apoptosis or autophagy. 
In conclusion, GBM in vitro studies indicate several important points. Firstly, ascorbate is capable of acting as a prooxidant in GBM cells in vitro. Secondly, there is much variation between cell lines, even amongst purportedly the same type of cancer. It cannot be assumed that one GBM cell line will react in the same way as others, so individual cell line tests will provide much needed information. Thirdly, some data suggest a useful distinction in vulnerability between normal and cancer cells, yet this must be further confirmed if ascorbate is to become a viable potential treatment.

\section{In Vivo Animal Model Trials}

Far fewer in vivo studies than in vitro have been carried out on the effect of ascorbate on cancer. Although a handful of in vivo studies have investigated the effect of oral ascorbate only on reducing tumours or improving other cancer outcomes (Varga and Airoldi 1983), these trials will not be discussed here, due to the tightly regulated pharmacokinetics and the conclusion that they cannot reach pharmacological concentrations. Oral ascorbate could potentially be effective in other capacities, such as immuno-stimulation and increasing the strength of extracellular matrix, yet it is not sufficient to reach the levels capable of incurring prooxidant activity.

Many of the following studies utilised intraperitoneal injections in animals to simulate intravenous dosing in humans. The mode of delivery influences the specific uptake of ascorbate, as has been already discussed, yet reports have validated this substitution with pharmacokinetic modelling. Chen et al. (2008) reported that in gavage (oral) dosing of rats, the pattern of ascorbate concentration over time mirrored that of intestinal absorption in humans. Further, intravenous administration of the same dose caused an 80 -fold higher concentration of ascorbate, approximately $8 \mathrm{mM}$, and intraperitoneal injections caused, at the highest peak, a 30-fold increase on gavage concentration. Although intravenous and intraperitoneal administration caused differing initial peak serum concentration, within 20 minutes the intravenous concentration stabilised, and the two methods followed a similar time/concentration pattern, albeit with intravenous delivery remaining slightly higher until the 90 -minute time point. Thus, it 
can be assumed that this method of delivery in mice is approximately equivalent to the intravenous infusions used in human studies.

Although the focus of this project is GBM, much more of the informative studies have been carried out in pancreatic models. There are several examples here of ascorbate successfully acting in a prooxidant role in this model. However, caution should be applied when considering the translation of pancreatic cancer to GBM, which is in a significantly different microenvironment.

Recently, Fukui et al. (Fukui, Yamabe et al. 2015) published evidence that ascorbate alone could decrease tumour growth in a nude mouse model of pancreatic cancer via strong oxidative stress, followed by an activation of proteins associated with autophagy and eventually cell death. The group discovered that, in line with their in vitro work involving MIA-PaCa-2 cells, an established pancreatic cancer model, nu/nu mice that were inoculated with these cells and then underwent thrice weekly ascorbate intraperitoneal injections from 3 weeks subsequently, had "significantly suppressed tumour growth and tumour weight."

GBM xenografts were treated with irradiation (2x 6 Gy), chemotherapy $(5 \mathrm{mg} / \mathrm{kg}$ temozolomide), and ascorbate $(2-5 \mathrm{mM})$ by Allen et al. Mice treated with both irradiation and chemotherapy had unsurprisingly longer survival than control, untreated mice, yet it was reported that the addition of ascorbate to the regimen could decrease tumour growth and so increase survival, in this case. Mice that received ascorbate in addition to traditional therapies for their GBM xenographs lived on average 2 weeks longer than mice that received only irradiation and chemotherapy.

Chen et al. used pancreatic murine xenographs to investigate the effect of ascorbate in combination with the other traditional antioxidant glutathione (Chen, Stone et al. 2011). They reported that $4 \mathrm{~g} / \mathrm{kg}$ of ascorbate daily reduced tumour volume by nearly half. Although all treatments, ascorbate, glutathione, and combination treatments improved survival 
compared to control mice, glutathione in fact abrogated the effectiveness of ascorbate alone, indicating a competitiveness between the two traditional antioxidants that is in stark contrast to their synergistic function outlined earlier.

The McConnell group's most recent paper (Grasso, Fabre et al. 2014) used a standard dose of irradiation in combination with ascorbate $(5 \mathrm{mM})$, and showed that pre-treatment with ascorbate for 1-hour radiosensitized the murine GL261 cells, as indicated by survival and clonogenic assays in vitro. However, in C57BL/6 mice with intracranial GL261 tumour cells, this radio-sensitising function of ascorbate was reversed.

Irradiation only mice were treated with a single dose of 4.5 Gy to the brain 8 days post intracranial surgery, ascorbate only mice received ascorbate daily via intraperitoneal injection from day 8 to day 45 after surgery, and the combined treatment mice received both treatments.

Although irradiation alone increased survival time, as expected, ascorbate only treated mice survived no longer than the control group. Most interestingly, the group of mice that received both treatments had in fact shorter survival than those that were given irradiation only. Histological analysis reported less tumour necrosis in this group also.

Essentially, ascorbate had inhibited the anti-tumorigenic effect of irradiation, indicating a potential, and dangerous radio-protective role. 


\section{Aims}

The overall aim of this thesis was to determine whether ascorbate has prooxidant effect in a brain tumour model in vivo. This research builds on previous work in our lab that demonstrates radiosensitisation of tumour cells in vitro by ascorbate, but expands knowledge by looking at a variety of specific DNA damage indicators in this process. Although survival has been assessed in in vivo GL261 models of ascorbate's interaction with irradiation in GBM, analysis of effects on DNA has not yet been determined, and so will be examined in this study.

In order to achieve this, the following specific objectives were proposed.

The first objective was to confirm the prooxidant activity on the in vitro GL261 model, and to determine the precise mode of DNA damage.

The second objective was to use a murine intracranial glioma model to investigate whether ascorbate led to the same DNA damage in vivo. 


\section{Methods}

\section{Materials}

Cell culture plastic consumables were purchased from In Vitro Technologies (Auckland, New Zealand); cell culture reagents were from Life Technologies (Carlsbad, CA., USA). Sodium ascorbate and other chemicals were from Sigma (St. Louis, MO., USA).

\section{Cell Lines}

The mouse GBM cell line, GL261, was from the NCI tumour cell line repository (Fredrick, MD., USA). Immortalized human GBM cell lines (LN18, T98G) were from the ATCC (Manassas, VA., USA). Primary GBM cell lines (NZG 1003, NZG 0809), were cultured from tumour tissue from patients undergoing debulking surgery, as previously described in Hunn, Farrand et al. 2012.

\section{Cell Culture}

Cells were kept in cell culture flasks, stored in incubators (Sanyo $\mathrm{MCO}-20 \mathrm{AIC} \mathrm{CO}_{2}$ Incubator) set to $37^{\circ} \mathrm{C}$, under $5 \% \mathrm{CO}_{2}$ and $95 \%$ humidity.

\section{Media}

DMEM (for GL261 cells), supplemented with:

500mL DMEM

$50 \mathrm{~mL}$ FCS $($ to $=\sim 9 \% \mathrm{v} / \mathrm{v})$

$5 \mathrm{~mL}$ Glutamax $(\mathrm{To}=\sim 1 \% \mathrm{v} / \mathrm{v})$

RPMI-1640 (for T98G, LN18, 1003, and 0809 cells), supplemented with:

500mL RPMI-1640

$30 \mathrm{~mL}$ FCS $($ to $=\sim 5.6 \% \mathrm{v} / \mathrm{v})$

\section{Treatments}

\section{Ascorbate}

Dehydrated sodium ascorbate

Diluted in PBS to a $1.5 \mathrm{M}$ working solution 
Added to final concentration of $5 \mathrm{mM}$

\section{Hydrogen Peroxide}

Stock solution $7.23 \mathrm{M}$

Diluted in PBS to a $7.23 \mathrm{mM}$ working solution

Added to final concentration of $500 \mu \mathrm{M}$

\section{Irradiation}

Cells were irradiated with Cesium-137 gamma-rays (Gammacell 3000 Elan, Best Theratronics) for the required time for a given dose. 1 minute of irradiation corresponded to approximately $4.2 \mathrm{~Gy}$, with slight variation over the course of the year as the radiation source lost potency.

\section{DNA Damage Analysis}

\section{Flow Cytometry}

In this investigation, the process for assessing the degree of $\mathrm{YH} 2 \mathrm{AX}$ and $8-\mathrm{OHdG}$ in cells between treatment groups was similar. Aside from different antibodies, the only other difference was the requirement of secondary 488 conjugated antibodies when measuring 8-OHdG.

GL261, 1003, or T98G cells were plated at $3 \times 10^{4}$ cells per well in 6well plates. 3 wells of each 6-well plate were assigned to treatment conditions, either control, ascorbate, irradiation, hydrogen peroxide, or both ascorbate and irradiation. The next day, 24 prior to treatment, media was removed from wells and replaced with fresh media. Cells were approximately $40 \%$ confluent and growing exponentially when treated.

Cells were treated with ascorbate to a total concentration of $5 \mathrm{mM}$ for 60 minutes. Dehydrated ascorbate was diluted with PBS (Dulbecco's Phosphate Buffer Saline, 1.4M NaCl, 27mM KCl, 170mM NaH${ }_{2} \mathrm{PO}_{4}$, 17.6mM KH $\mathrm{KH}_{2} \mathrm{PO}_{4}$ ) fresh for each experiment. 7.23M stock hydrogen peroxide was diluted with PBS and added to wells for a final concentration of $500 \mu \mathrm{M}$. 
60 minutes after ascorbate or hydrogen peroxide treatment of cells, media was aspirated from wells and plates were irradiated for 1 minute and 25 or 26 seconds, for a final dosage of $6 \mathrm{~Gy}$, or longer depending on dosage required.

For anti- $\mathrm{YH} 2 \mathrm{AX}$ antibody assays, wells were replaced with fresh media and kept in an incubator set to $37^{\circ} \mathrm{C}$ for a further 60 minutes before beginning harvest. Cells were fixed at 180 minutes' post-irradiation. In the case of anti-8-OHdG assays, cells were harvested immediately postirradiation in order to complete fixation within 60 minutes of irradiation treatment.

To harvest cells for flow cytometry analysis, wells were aspirated, rinsed with PBS, and incubated with $1 \mathrm{~mL}$ of TE for 4 minutes at $37^{\circ} \mathrm{C}$. TE was quenched with $2 \mathrm{mLs}$ of media and cells were collected in $15 \mathrm{~mL}$ Falcon tubes, spun for 5 minutes at 500g in a centrifuge (Gryozen 1580MGR), and distributed with $200 \mu \mathrm{L}$ of PBS into a round bottomed 96 well plate. Cells were washed in PBS with 1\% BSA (FACs buffer), and then fixed with 100 $\mu \mathrm{L}$ of FoxP3 fixative for 30 minutes at $4^{\circ} \mathrm{C}$. After fixation, cells were spun for 8 minutes at $800 \mathrm{~g}$, washed twice in $200 \mu \mathrm{L}$ of BD Perm/Wash buffer, incubated at $4^{\circ} \mathrm{C}$ for 15 minutes, before being pelleted and resuspended in $50 \mu \mathrm{L}$ of the required concentration of antibody solution at $4^{\circ} \mathrm{C}$ overnight.

For anti-rH2AX antibody assays, antibody (Alexa Fluor ${ }^{\circledR} 488$ antiH2A.X-Phosphorylated [Ser139] 2F3 Clone, mouse IgG1 isotype, Biolegend) was diluted in BD Perm/Wash at a ratio of 1:150 (stock concentration $25 \mathrm{ug} / \mathrm{mL}$, working concentration $166.7 \mathrm{ng} / \mathrm{mL}$ ). Approximately 15 hours later, antibody was removed, cells were again washed twice in BD Perm/Wash buffer and resuspended in $300 \mu \mathrm{L}$ of FACs buffer for flow cytometry analysis;

For anti-8-OhdG assays, primary antibody (8-OHdG [J-1]: sc139586, Santa Cruz) was diluted in BD Perm/Wash at 1:250 (stock 
concentration $100 \mathrm{ug} / \mathrm{mL}$, working concentration $400 \mathrm{ng} / \mathrm{mL}$ ), and isotype controls were diluted to the same concentration as each primary antibody in BD Perm/Wash. For 8-OhdG tests, cells were incubated in secondary antibody (Goat Polyclonal Anti-Rabbit IgG H\&L [Alexa Fluor® 488], Abcam) at 1:2000 (stock concentration $2000 \mathrm{ug} / \mathrm{mL}$, working concentration $1000 \mathrm{ng} / \mathrm{mL}$ ) for an hour at room temperature, before antibody was removed, cells were washed twice in BD Perm/Wash, and resuspended in $300 \mu \mathrm{L}$ of FACs buffer for analysis.

Cellular fluorescence was measured by flow cytometric analysis, carried out on a BD FACS Canto TM II, with BD FACS Diva Software Version 8.0.1. Analysis of flow cytometry data was carried out on FlowJo 10.0.8r1 (FlowJo, LLC, Ashland, OR, USA). Statistical analysis of FlowJo outputs was carried out on Prism 6.0 (GraphPad Software, La Jolla, CA, USA).

\section{Comet Tail Assay/Single Cell Gel Electrophoresis}

Slides used for the comet tail assay (LabServ Superfrost Plus) were pre-coated with $1 \mathrm{~mL}$ of $1 \%$ normal melting point agarose (Invitrogen UltraPure Agarose) and allowed to air-dry for at least 24 hours prior to the start of the assay. Slides were coded with an individual serial number, which was randomly assigned to treatment groups and cell lines prior to the start of the experiment. Slides were only decoded after analysis.

GL261 cells were harvested and diluted in media to a concentration of $1 \times 10^{5}$ cells $/ \mathrm{mL}$, then treated with 6 Grays of ionising radiation, $5 \mathrm{mM}$ of ascorbate or both agents together. Ascorbate treated cells were pre-treated with ascorbate in media for an hour before irradiation.

Cells were added to $1 \%$ low melting point agarose at a ratio of $1: 10$ ( $\mathrm{v} / \mathrm{v}$, final cell concentration $1 \times 10^{4}$ cells $/ \mathrm{mL}$ ), pipetted several times to combine, and then released onto a pre-agarose coated slide, in two isolated circular areas for each slide. 
Agarose and cells were allowed to air dry in a laminar flow hood for 30 minutes at room temperature.

Cells were lysed by placing slides in pre-chilled lysis solution $(2.5 \mathrm{M}$ $\mathrm{NaCl}$, [Sigma Aldrich] 100mM EDTA pH 10 [Invitrogen], 10mM Trizma [Sigma], 1\% sodium lauryl sarcosinate [Aldrich], and 1\% Triton X-100 [Bio-Rad], $\mathrm{pH} 10$ ) for 1 hour at $4^{\circ} \mathrm{C}$.

Slides were rinsed in $1 \mathrm{x}$ TBE, before being placed in a gel electrophoresis tank (Bio-Rad) containing 1x TBE and equilibrated for 30 minutes. The agarose-embedded cells were then electrophoresed (Amersham Pharmacia Biotech EPS-301 Electrophoresis Power Supply, 3 $\mathrm{V} / \mathrm{cm}$ for 60 minutes)

Slides were stained with $10 \mathrm{ug} / \mathrm{mL}$ propidium iodide pipetted directly onto agarose for 20 minutes at $4^{\circ} \mathrm{C}$, rinsed in TBE, and imaged on a fluorescent microscope (Olympus BX51 microscope, FluorView software, X-Cite Series 120Q mercury lamp, Olympus DP70 camera, TXRED filter). Slides were imaged at the longest exposure possible without incurring background stain, and exposure was kept identical within each experiment.

Cell nuclei were analysed using Robert Bagall's ImageJ Comet Assay plugin. Briefly, oval selections were used to outline and measure the cell nuclei head only, followed by the entire cell nuclei including the comet tail. Cell nuclei that overlapped, or were not entirely contained within one picture were not analysed. At least 100 cell nuclei per sample per experiment were analysed. ImageJ outputs (Table 3) were imported into Microsoft Excel for collection by experiment, and averages were imported into Prism (GraphPad) V6.0 for analysis. 


\begin{tabular}{|l|l|}
\hline $\begin{array}{l}\text { ImageJ parameter } \\
\text { output }\end{array}$ & Description \\
\hline $\mathrm{X}$ & X coordinate for each centroid \\
\hline $\mathrm{Y}$ & Y coordinate for each centroid \\
\hline XM & X coordinate for each center of mass \\
\hline $\mathrm{XY}$ & Y coordinate for each center of mass \\
\hline IntDen & $\begin{array}{l}\text { Integrated Density: the product } \\
\text { of 'Area' and 'Mean Gray Value' }\end{array}$ \\
\hline RawIntDen & $\begin{array}{l}\text { Raw Integrated Density: the sum of the values } \\
\text { of the pixels in the image or selection. }\end{array}$ \\
\hline TailLen & $\begin{array}{l}\text { Tail Length: the distance from the Centroid of } \\
\text { the head to the Center of Mass of the tail. }\end{array}$ \\
\hline TailMoment & $\begin{array}{l}\text { Tail Moment: the length of the tail times the } \\
\text { integrated density of the tail }\end{array}$ \\
\hline \%TailDNA & $\begin{array}{l}\text { Percentage Tail DNA: the integrated density } \\
\text { of the tail divided by the integrated density of } \\
\text { the tail plus the integrated density of the head } \\
\text { times 100. }\end{array}$ \\
\hline $\begin{array}{l}\text { Information from } \\
\text { (University of North Carolina School of Medicine 2015) } \\
\text { (Research Services Branch) }\end{array}$ \\
\hline
\end{tabular}

Note in this analysis, measurement of the nucleoid 'tail' always included measurement of the nucleoid 'head' as well, thus 'integrated density of the tail' could be more accurately termed integrated density of whole comet (Figure 22). This formula results in a range of 50\% (where comet head integrated density $=$ whole comet integrated density, i.e. no visible tail) to approaching $100 \%$ (100\% tail DNA is a hypothetical maximum whereby no comet tail head would be visible).

\section{Intracranial Mouse Model}

Experiments using mice were conducted in accordance with the New Zealand Animal Welfare Act 1991. All protocols were approved by the Victoria University Animal Ethics Committee (Number 2012R18M) and done according to their guidelines.

The inbred mouse strain C57BL/6 was originally procured from the Animal Resource Centre (Canning Vale, WA). Mice were managed by the 
Biomedical Research Unit at the Malaghan Institute of Medical Research (Wellington, New Zealand). All mice were males 8 to 12 weeks of age.

Approximately $1 \times 10^{6}$ mouse glioma GL261 cells, stored frozen in liquid nitrogen, were thawed, centrifuged at $300 \mathrm{~g}$ for 5 minutes, washed in PBS, and a subset implanted into the brain of 8 to 12 week old (25-30 g) male C57BL/6 mice, following the procedure described by Broadley, Hunn et al. 2011.

Mice were anesthetized by intraperitoneal (I.P.) injection (UltraFine Insulin syringe $1 \mathrm{~mL}, 29 \mathrm{G}, \mathrm{BD})$ of xylazine $(100 \mathrm{mg} / \mathrm{kg})$ and ketamine (10 $\mathrm{mg} / \mathrm{kg}$ ) (Phoenix Pharm). Once unconscious, mice were given a subcutaneous injection of buprenorphine analgesic $(0.1 \mathrm{mg} / \mathrm{kg}$, Renckitt Benckiser Pharmaceuticals), Lacri-Lube (Allergan) was applied across the eye and mice were restrained in a stereotaxic frame (Stoelting Apparatus).

The skin and fur of the head were disinfected with an iodine covered swab, and an incision was made approximately $1.5 \mathrm{~cm}$ along the midline with a fresh scalpel (Swann Morton). A burr hole was drilled (Terumo Needle, 20G, Neolus) in the skull $1 \mathrm{~mm}$ posterior to the bregma and $2.3 \mathrm{~mm}$ lateral to the midline (Figure 6).

Cells $\left(1.25 \times 10^{7} / \mathrm{mL}\right.$ of PBS $)$ were inoculated via the burr hole using a 32-gage needle and Hamilton syringe. The needle was slowly advanced to a depth of $4 \mathrm{~mm}$ from the brain surface, withdrawn $1 \mathrm{~mm}$, and $2 \mu \mathrm{L}$ of cell suspension delivered over the course of approximately $1 \mathrm{~min}$. The needle was left in place for 3-5 min, and then raised slowly. The burr hole was sealed with bone wax and the incision sutured (Novafil 18" Surgalloy P-10, Covidien)

Following surgery, (immediately following, 24, and 48 hours postsurgery) animals received sub-cutaneous Carprofen $(5 \mathrm{mg} / \mathrm{kg}$, Norbrook Laboratories), for treating pain. 
Animals were weighed at regular intervals and humanely sacrificed by $\mathrm{CO}_{2}$ when weight loss occurred ( $>10 \%$ of body weight) or at the experimental endpoint; either day 18 for short-term treatment, or day 19 for longer-term treatment. 


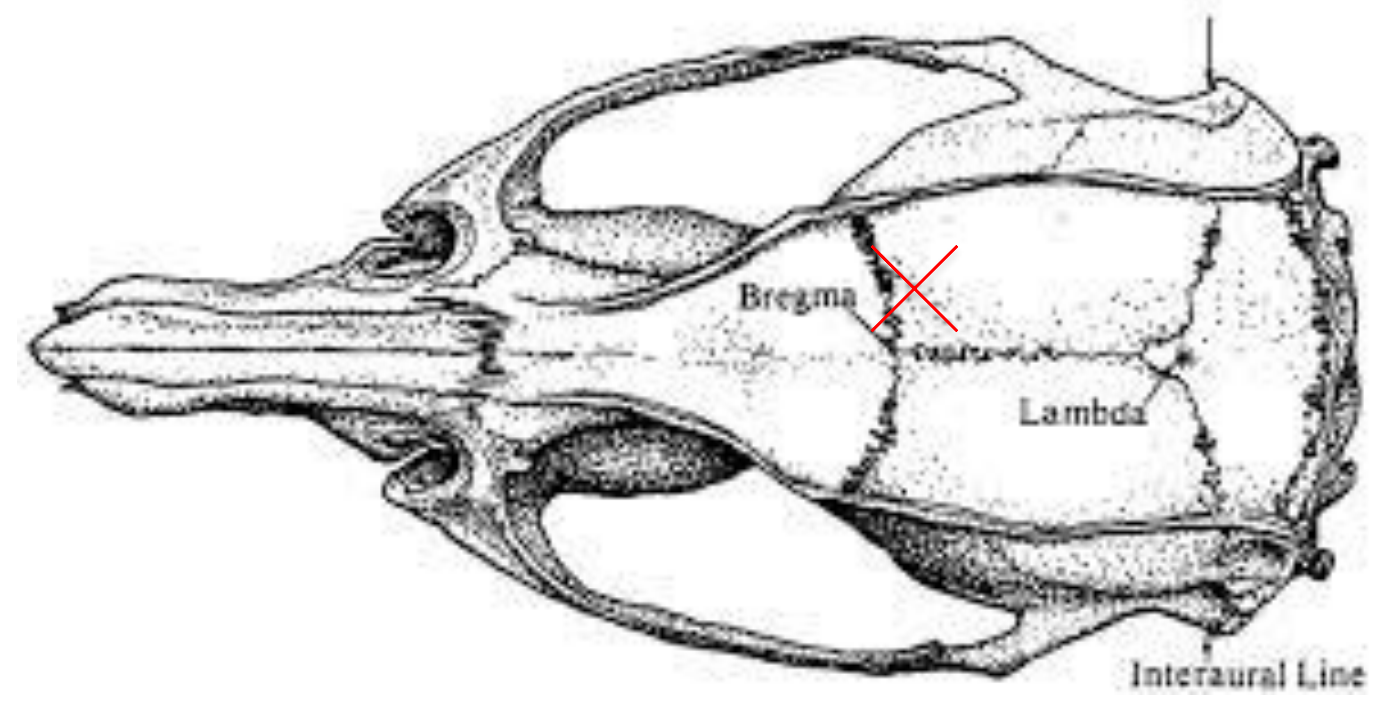

Figure 6. Injection site of GBM cells in the mouse skull. Red X represents site of injection.

Image used with permission from (Paxinos and Watson 1998). This article was published in The Rat Brain in Stereotaxic Coordinates, 4th Edition, George Paxinos and Charles Watson, Stereotaxic Reference System, pg. XIV to XIV, Copyright Elsevier (1998). 


\section{Whole Brain Irradiation of Mice}

Mice received a single dose of 10 Gy to the brain on day 18 or 19 after surgery. The irradiator uses a Cesium-137 source (length $27 \mathrm{~cm}$, diameter $1.7 \mathrm{~cm}$; nominal activity $48 \mathrm{TBq})$.

Mice were anesthetized with an intraperitoneal (I.P.) injection of xylazine $(100 \mathrm{mg} / \mathrm{kg})$ and ketamine $(10 \mathrm{mg} / \mathrm{kg})$. Once unconscious, Lacrilube was spread across the cornea and mice were placed upright in a $50 \mathrm{~mL}$ Falcon tube with a hole at the tip for breathing, and tissue paper at the base to prevent movement. The tube was placed inside a $2 \mathrm{~cm}$ thick custom-built lead shield, such that only the mouse's head above the ears is exposed to radiation. The shielding was placed in the centre of the steel cylinder (Figure 7).

\section{Tumour Harvest}

Mice were culled with a $\mathrm{CO}_{2}$ chamber, then perfused with $10 \mathrm{~mL}$ of PBS and 10mL of $10 \%$ neutral buffered formalin. Tumour-containing brains were transferred into a $15 \mathrm{~mL}$ Falcon tube that contained $3 \mathrm{~mL}$ of $10 \%$ neutral buffered formalin and fixed overnight (approximately 18 hours) at $4^{\circ} \mathrm{C}$ before being transferred into $4 \mathrm{~mL}$ of $70 \%$ molecular grade ethanol and stored at $4{ }^{\circ} \mathrm{C}$ until processing. Total time between tumour harvest and processing was less than 2 weeks.

\section{Tissue Processing and Immunofluorescence Assay}

Brains were bisected by hemisphere and dehydrated through a series of ethanol concentrations, xylol and wax (Table 4) with an automated tissue processor (Leica TP1020, Leica Biosystems). 
Table 4. Automated Tissue Processor Program

\begin{tabular}{|l|l|}
\hline Reagent & Time \\
\hline $70 \%$ Ethanol & 2 hours \\
\hline $80 \%$ Ethanol & 1 hour 30 minutes \\
\hline $95 \%$ Ethanol & 1 hour \\
\hline $95 \%$ Ethanol & 1 hour \\
\hline Absolute Ethanol & 1 hour 30 minutes \\
\hline Absolute Ethanol & 1 hour 15 minutes \\
\hline Absolute Ethanol & 1 hour 15 minutes \\
\hline $1: 1$ Absolute Ethanol/Xylol & 1 hour 30 minutes \\
\hline Xylol & 1 hour \\
\hline Xylol & 1 hour \\
\hline Wax & 1 hour 30 minutes \\
\hline Wax (with vacuum) & 2 hours \\
\hline
\end{tabular}

Hemispheres were set in paraffin wax with a paraffin embedding machine (Leica EG1160, Leica Biosystems); 5 micron slices were sectioned from the block with a microtome (Leica RM2235, Leica Biosystems), transferred via a waterbath (Leica HI1210, Leica Biosystems), and dried on SuperFrost slides.

Slides were stored at $4^{\circ} \mathrm{C}$ until staining.

Slides were rehydrated in a series of xylene and ethanol steps (Table $5)$.

Table 5. Immunofluorescence Protocol

\begin{tabular}{|l|l|}
\hline Reagent & Time \\
\hline Xylene & $2 \times 10$ minutes \\
\hline Absolute Ethanol & $4 \times 2$ minutes \\
\hline $90 \%$ Ethanol & $4 \times 2$ minutes \\
\hline $70 \%$ Ethanol & $4 \times 2$ minutes \\
\hline $\mathrm{ddH}_{2} \mathrm{O}$ & $4 \times 2$ minutes \\
\hline $10 \mathrm{mM}$ Sodium Citrate buffer, pH 6, boiling & $\begin{array}{l}10 \text { minutes at } 95^{\circ} \mathrm{C} \\
+20 \text { minute cooling }\end{array}$ \\
\hline TBST rinse & $\begin{array}{l}3 \times 5 \text { minutes } \\
1 \text { hour at room } \\
\text { temp. }\end{array}$ \\
\hline Primary antibody in 1\% BSA (in TBST) & $\begin{array}{l}1 \text { hour at room } \\
\text { temp. }\end{array}$ \\
\hline TBST rinse & $3 \times 5$ minutes \\
\hline DAPI Anti-fade mounting media, coverslip & \\
\hline
\end{tabular}


Slides were imaged on a fluorescent microscope (Olympus BX51 microscope, FluorView software, X-Cite Series 120Q mercury lamp, Olympus DP70 camera, DAPI filter for nuclear imaging, FITC filter for Alexa Fluor 488 tagged $\mathrm{IH}_{2} \mathrm{AX}$ and 8-OH, and $\mathrm{Cy} 5$ filter for Alexa Fluor 647 tagged $\mathrm{YH} 2 \mathrm{AX}$ ) and analysed with ImageJ. The entirety of one brain hemisphere slice in each sample mouse, including regions of tumour, was observed in each immunofluorescent experiment.

\section{Statistical Analysis}

Graphed values are averages plus or minus statistical error of the mean (SEM). The statistical significance of differences between treatments was determined by paired (mice weights) or unpaired (flow cytometry and comet tail assays) two-tailed Student $t$ tests; a $p$ value less than 0.05 was considered statistically significant. 

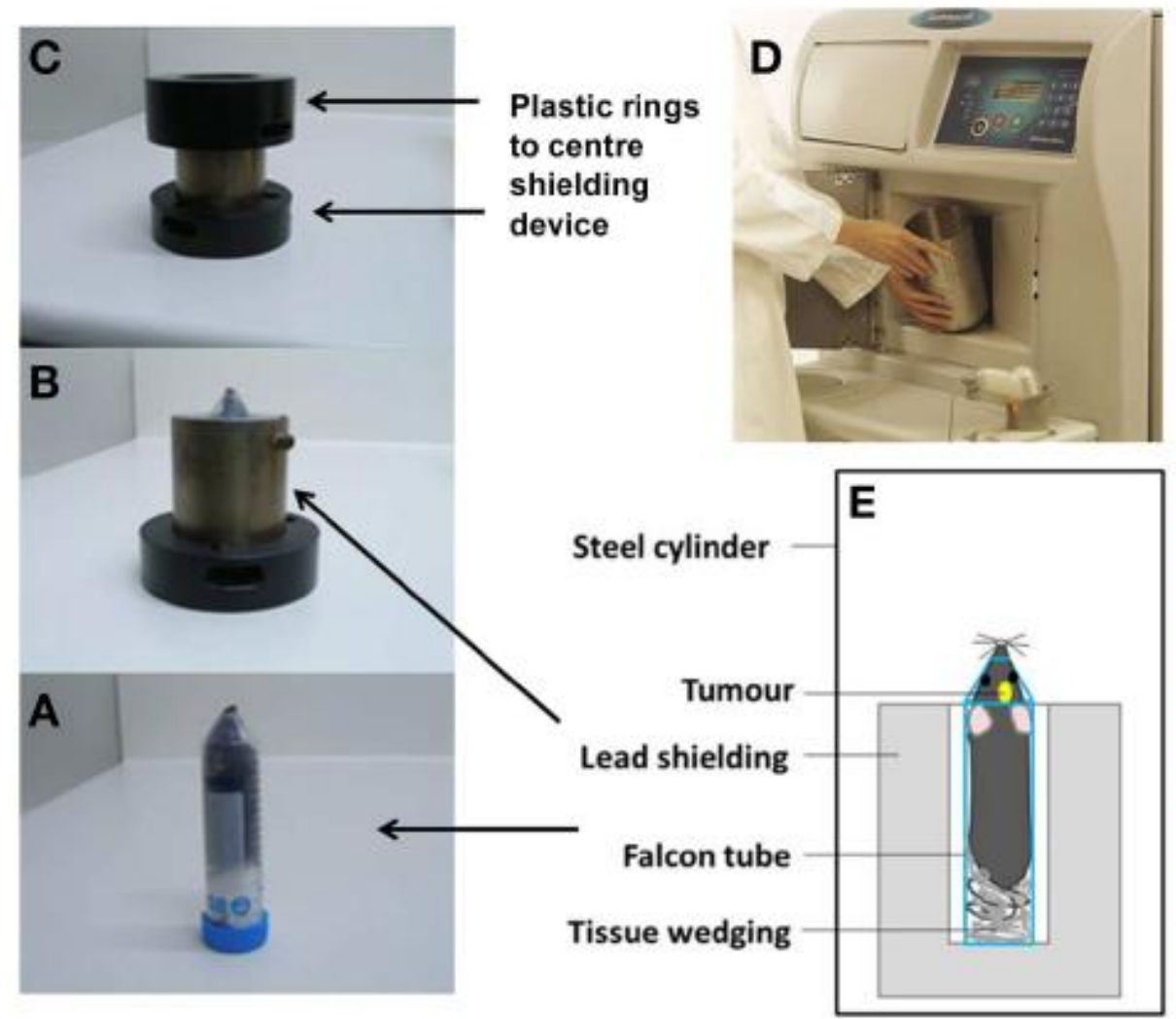

${ }^{137}$ Cesium
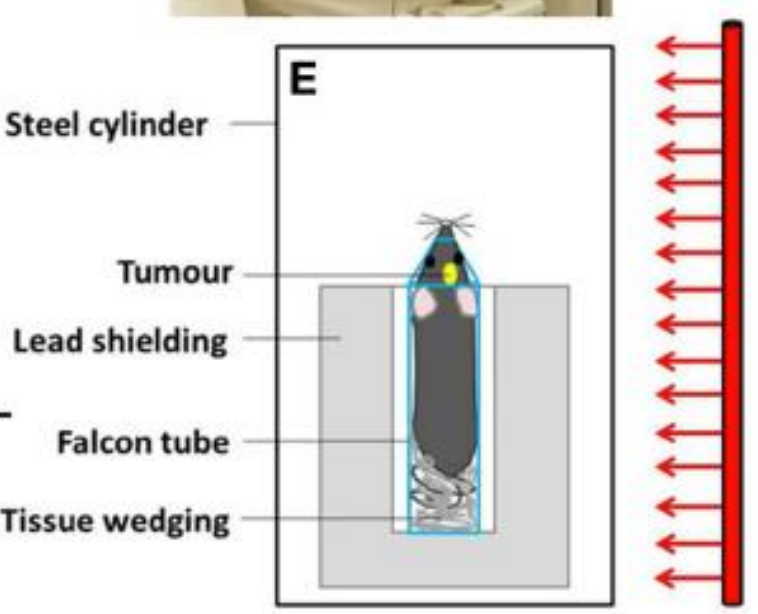

FIGURE 1 | Irradiation setup for whole brain irradiation of mice. The mouse is positioned in a Falcon tube (A), inside a $2 \mathrm{~cm}$ thick lead shielding device (B) inside plastic rings (C) inside the aluminum cylinder in the Gamma cell irradiator (D,E). Diagrammatic representation of the irradiation setup.

Figure 7. A graphical representation of whole brain mouse irradiation

From (Grasso, Fabre et al. 2014) 


\section{Results}

The ultimate aim of this project was to determine whether ascorbate has any pro-oxidant activity in a brain tumour model. Pro-oxidant activity would lead to radio-sensitisation and determine the suitability of ascorbate as a radiotherapy adjuvant in GBM cancer therapy.

\section{Analysis of DNA Damage In Vitro}

The first objective of the project was to confirm whether ascorbate had radiosensitisation activity in vitro on GL261 cells, a murine model of GBM and the same cells that would be used in vivo. To this end, flow cytometry and the single cell gel electrophoresis assay were used to determine whether ascorbate induced oxidative damage and double stranded DNA breaks in GL261 cells.

\section{Flow Cytometry}

Cells were plated, and at $40 \%$ confluence were treated with either 6 Gy of irradiation, $5 \mathrm{mM}$ of ascorbate for $60 \mathrm{~min}, 500 \mu \mathrm{M}$ of hydrogen peroxide for the same length of time, or both ascorbate and irradiation. For cells that received both ascorbate and irradiation, ascorbate was given as a pre-treatment 60 minutes prior to irradiation. After irradiation, cells were harvested before being processed for analysis using an antibody to either ҮH2AX or 8-OHdG.

Figure 8 describes the flow cytometric analysis including gating procedures used throughout. Each experiment was grouped by cell line and concentration of antibody. Within each group, cells in the control sample was gated on FSC (forward scatter) and SSC (side scatter) to eliminate debris, and the gate was copied to every other sample within that group. Single cell events were identified using forward scatter area (FSC-A) and forward scatter height (FSC-H), and gated to eliminate double cells (doublets). Finally, a quadrant gate was inserted to the right of the main population of control cells in order to measure the proportion of cells that were more fluorescent than the control population. 

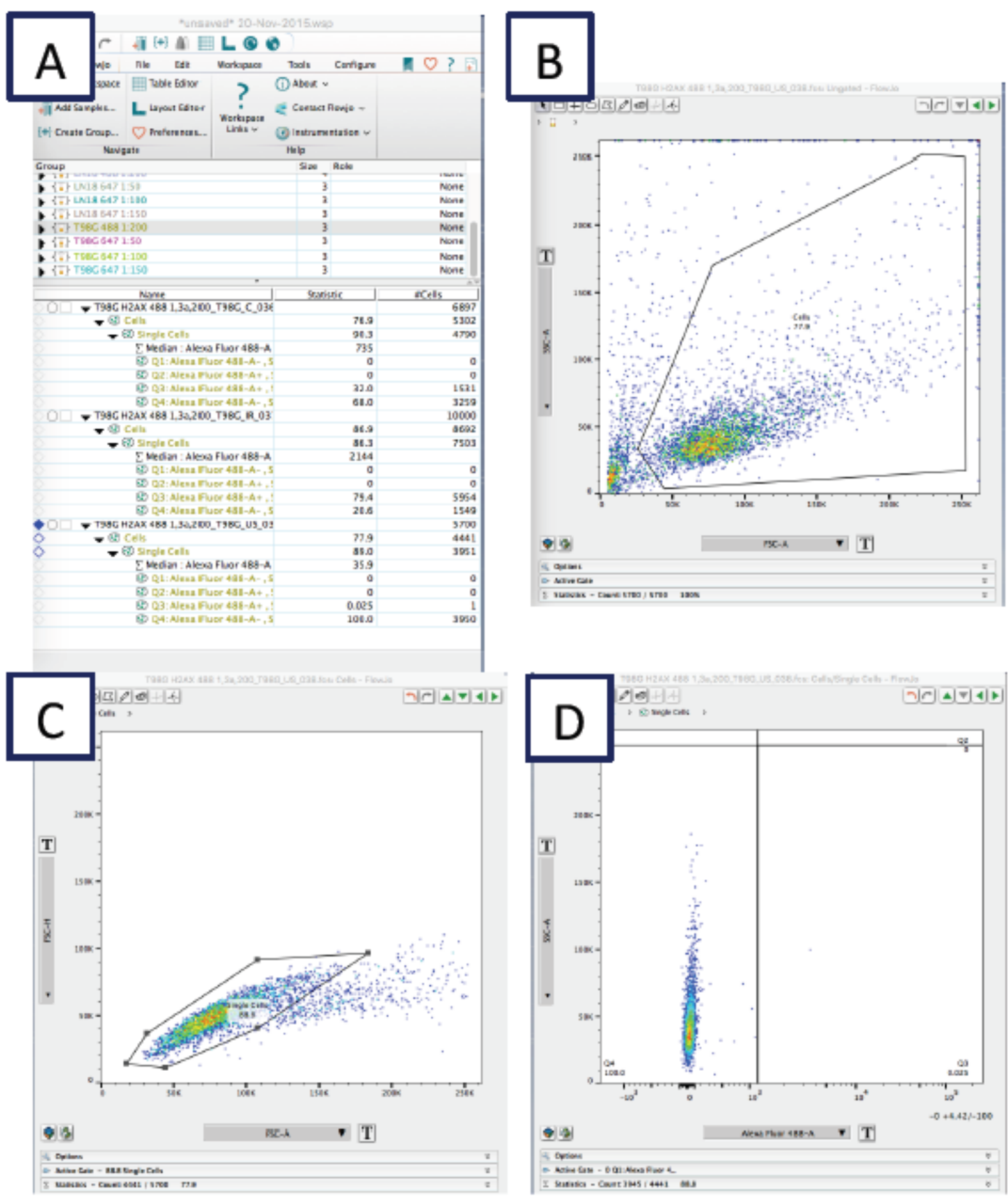

Figure 8. Example representative analysis of flow cytometry cell data

Specific analysis displayed is of unstained T98G cells. Cells were plated, treated, and harvested before being stained with antibody for a specific marker. Fluorescence was measured by BD FACS Canto TM II, with BD FACS Diva Software Version 8.0.1. A. Data was imported into FlowJo 10.0.8r1 and grouped by cell line, fluorophore marker, and antibody concentration. B. Cells were separated from debris by gating on side scatter light area (SSC-A) and forward scatter light area (FSC-A). C. Single cells were separated from doublet cells by gating on forward scatter light height (FSC-H) and FSC-A. D. Cells were graphed on SSC-A and the fluorophore marking the protein of interest (in this case Alexa Fluor 488-A indicating $\mathrm{YH} 2 \mathrm{AX}$ ). Median fluorescence intensity was determined by the median fluorophore value on the $\mathrm{x}$ axis. Percent positive was determined by the percentage of cells to the right of a gate inserted relative to control cells. 
Figure 9 displays a typical graphical output for one experiment. Unstained negative control and isotype control cell populations are nearly entirely, or entirely to the left of the final quadrant gate, with no positive fluorescence. Control cells serve as a marker of the basal level of DNA damage, and treated cell populations are shifted to the right relative to the control population, indicating an increase in fluorescence, thus an increase in the proportion of cells positive for the marker investigated. This particular experiment displays $\mathrm{rH} 2 \mathrm{AX}$ fluorescence in response to ascorbate, irradiation, and combination treatments. The combination of irradiation and ascorbate has more cells with bright $\Upsilon \mathrm{H} 2 \mathrm{AX}$ staining ( shifted to the right more strongly) than either treatment alone.

\section{ҮH2AX}

YH2AX is a modified histone marker of DNA damage, an instigator of the DNA damage response, and often interpreted as a marker of DSBs. DSBs are an important indicator because they are considered to be the most damaging DNA alteration, and most likely to cause cell death, because they are the most difficult to repair correctly. Even if it does not indicate only DSBs (see Takahashi and Ohnishi 2005), DNA damage in general is also an indicator of the cell's likelihood of death, for an accumulation of any DNA damage over a threshold will lead to cell death.

In this investigation, intensity of $\Upsilon \mathrm{H} 2 \mathrm{AX}$ was used in vitro as one indication of the type of damage that can be caused by ascorbate and irradiation, in order to corroborate similar data from human cell lines (Castro, McConnell et al. 2014). 


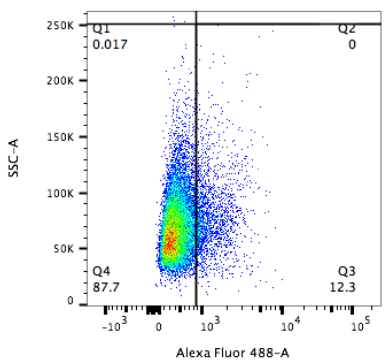

CL261_H2AX_CL261_C_003.fCS Single Cells
17826

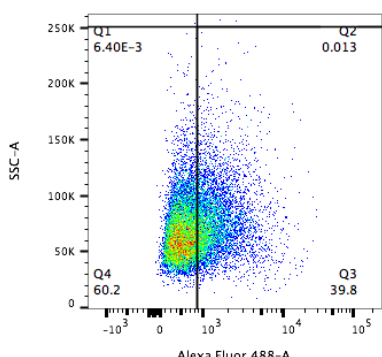

GL261_H2AX_CL261_AA_004.fCs Single C

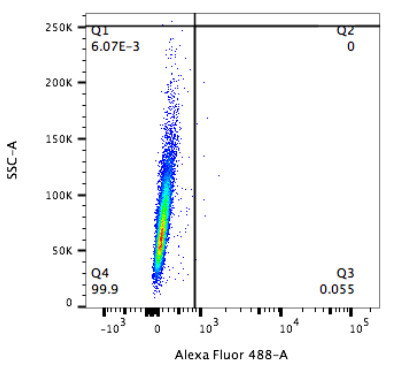

CL261_H2AX_CL261_US_001.fCs Single Cells

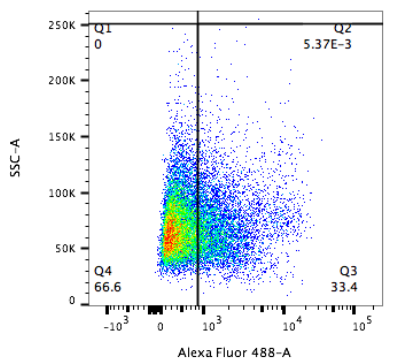

CL261_H2AX_CL261_CY_005.fcs Single $C$

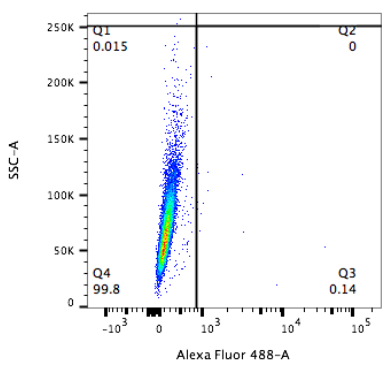

GL261_H2AX_G1261_ISO_002 fCS Single Cell
12959

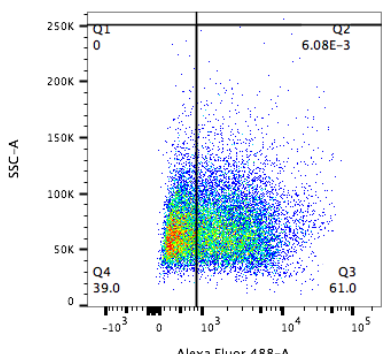

GL261_H2AX_CL261_AA+CY_006.fCs Single Cells

Figure 9. Representative example of $\mathrm{YH2AX}$ analysis in GL261 cells in response to treatment

Specific analysis is of $\mathrm{YH} 2 \mathrm{AX}$ staining of GL261 cells in response to various treatments. Plotted in FlowJo 10.0.8r1. Left to right, top row: Control, Unstained negative control, Isotype negative control. Left to right, second row: Ascorbate treated, Irradiation treated, both Ascorbate and Irradiation treated. Cells were stained, measured and analysed as described in Figure 8. Cells were plotted by SSC-A and fluorophore indicating protein marker of interest, and gated relative to untreated control cells. The number in the bottom right hand side of each graph indicates the percentage of total cells where antibody staining exceeded the gate. The gate was inserted according to the position of control untreated cells and applied to all other treatments. 
Figure 10 shows YH2AX levels in GL261 cells in response to irradiation, ascorbate, and both treatments in combination. Each sample was gated as described in Figure 8. The effect of each treatment on $\mathrm{YH} 2 \mathrm{AX}$ was calculated as percent positive cells, meaning the percentage of cells to the right of the gate as shown in Figure 9. Each treated cell was normalised to control, untreated cells then calculated as a fold change. The data show that while ascorbate increased the proportion of $\Upsilon \mathrm{H} 2 \mathrm{AX}$ positive cells nearly 3fold, irradiation increased it nearly 3.1 fold, and both irradiation and ascorbate together caused $\Upsilon \mathrm{H} 2 \mathrm{AX}$ percentage positive staining to increase nearly 4-fold. The increase in $\mathrm{rH}_{2} \mathrm{AX}$ with both treatments is thus less than additive.

Counting the number of positive cells is not necessarily informative. While the number of cells positive for a marker may not change, the amount of signal per cell can change, and changes in the level of signal in a cell is equally biologically relevant. The signal per cell is directly measured, and the effect on a population of cells calculated by analysis of the Median Fluorescence Intensity (MFI) of that population, along a fluorescence axis.

The MFI for YH2AX in GL261 cells in response to irradiation, ascorbate, and both treatments in combination is shown in Figure 11. Irradiation increases the $\mathrm{rH} 2 \mathrm{AX}$ staining per cell, compared to control, 5.8fold. Ascorbate increases that metric only 3.2-fold, less than irradiation, and combination treatment increases $\mathrm{YH}_{2} \mathrm{AX}$ more than either treatment alone, 12.2-fold.

The effect of combined treatment was greater in the amount of ҮH2AX per cell (MFI) than in the number of $\Upsilon^{\mathrm{CH}} 2 \mathrm{AX}$ positive cells, and results in an apparently synergistic interaction between ascorbate and irradiation in this case. 
$\gamma$ H2AX Percent Positive GL261

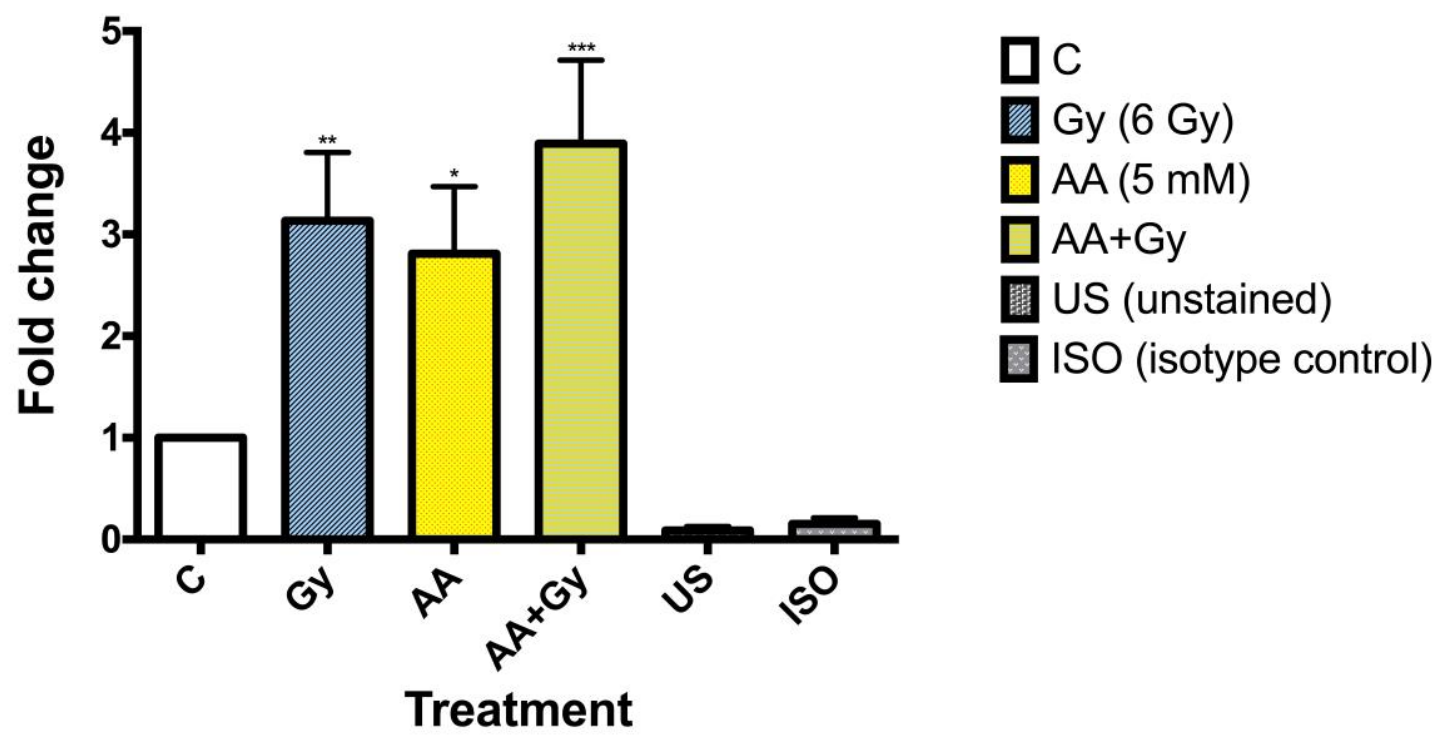

Figure 10. Flow cytometry derived percent positive $\mathrm{YH} 2 \mathrm{AX}$ staining in GL261 cells

Flow cytometry derived percent positive $\mathrm{rH} 2 \mathrm{AX}$ staining (fold change + Standard Error of the Mean [SEM]) by treatment group for the GL261 cell line. Graph includes data from 8 independent experiments, and each treatment group has a sample size of between 5 and 8 experiments. All three treatment groups were statistically significantly greater than control (P values: Gy 0.0046, AA 0.0158, AA+Gy 0.0008). Graph and statistics produced with Prism V6.0. 
$\gamma \mathrm{H} 2 \mathrm{AX}$ Median Fluorescence Intensity

GL261

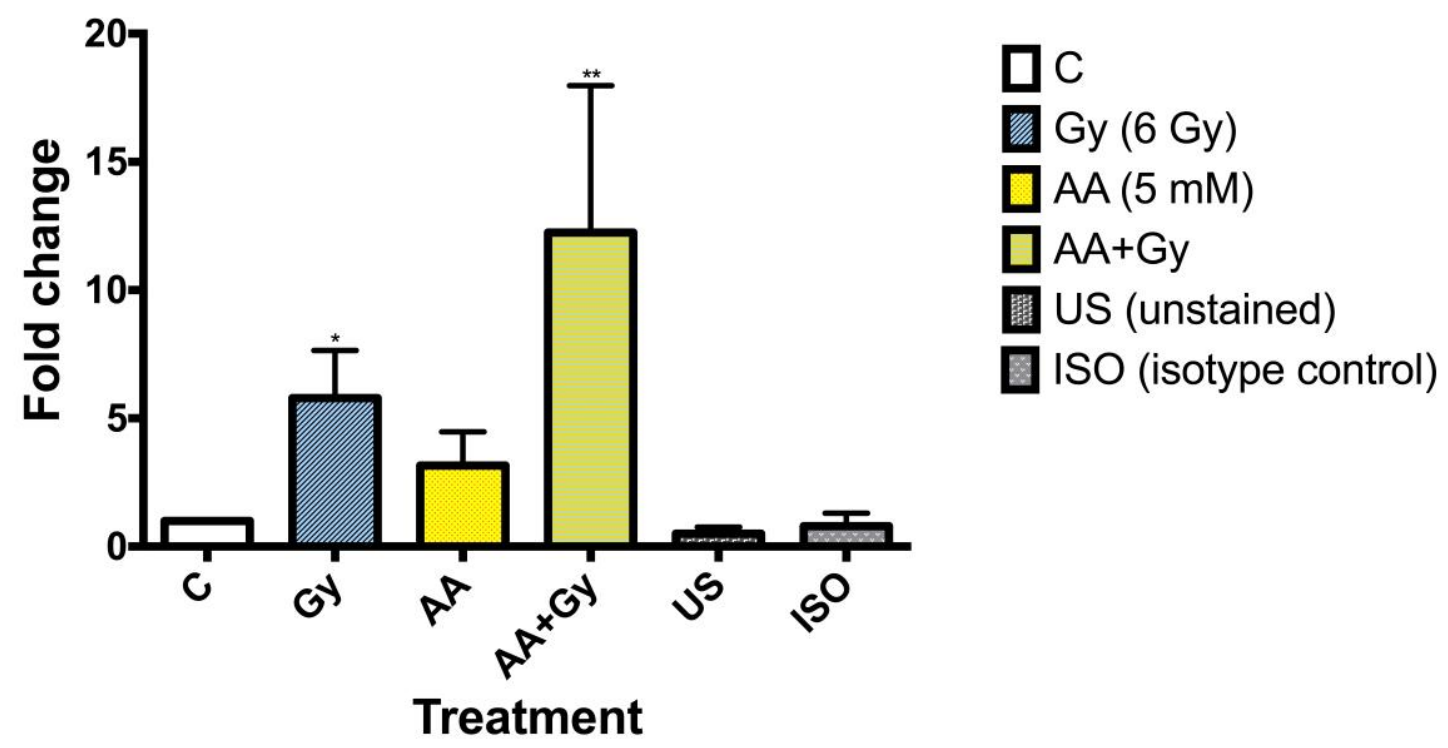

Figure 11. Flow cytometry derived median fluorescence intensity YH2AX staining in GL261 cells

Flow cytometry derived median fluorescence intensity YH2AX staining (fold change + Standard Error of the Mean [SEM]) by treatment group for the GL261 cell line. Graph includes data from 8 independent experiments, and each treatment group has a sample size of between 5 and 8 experiments. Irradiation and combination treatment groups were statistically significantly greater than control (P values: Gy 0.0156, AA 0.1201, AA+Gy 0.0267). Graph and statistics produced with Prism V6.0 
These experiments were repeated with a human GBM cell line, to determine how well G1261 represents human glioma cells. The T98G cell line is an immortalised human GBM-derived cell line commonly used in brain cancer research. It is adherent like GL261, and in our laboratory is the most ascorbate resistant GBM cell line (Castro, McConnell et al. 2014).

Figure 12 presents the effect of ascorbate, irradiation and combination treatments on $\mathrm{YH} 2 \mathrm{AX}$ staining in $\mathrm{T} 98 \mathrm{G}$ cells displayed as fold change percent positive. With this cell line, irradiation only caused the greatest $\mathrm{\Upsilon H} 2 \mathrm{AX}$ increase, nearly 4-fold in comparison with control, while ascorbate only caused a minimal increase, around 1-fold. This corroborates a previous paper that found T98Gs had a much greater antioxidant capacity than other GBM cell lines (Castro, McConnell et al. 2014). Interestingly in this case, combination treatment seemed to cause less $\mathrm{\Upsilon H} 2 \mathrm{AX}$ positive staining than irradiation only, with just over 2.5 -fold more $\mathrm{rH} 2 \mathrm{AX}$ positive staining.

In Figure 13, T98G, like GL261, is assessed with MFI to complement percent positive display. MFI shows ascorbate caused a similar, minimal fold change increase in $\mathrm{rH} 2 \mathrm{AX}$ signal of T98G cells compared to control, and irradiation only resulted in about 5-fold amplification, comparable to the percent positive data. Most interestingly, in comparison with the previous graph of percent positive cells, combination treatments were additive, with a greater $\Upsilon \mathrm{H} 2 \mathrm{AX}$ positive signal compared with either irradiation or ascorbate alone.

This data shows that irradiation alone caused a greater percentage of the total cell population to fluoresce positive for $\Upsilon \mathrm{H} 2 \mathrm{AX}$, and ascorbate appeared to decrease this proportion in combined treatments. Yet, looking at the combination fluorescence with MFI, both treatments caused more fluorescence per individual cell compared with either ascorbate or irradiation alone. 
$\gamma \mathrm{H}$ 2AX Percent Positive T98G

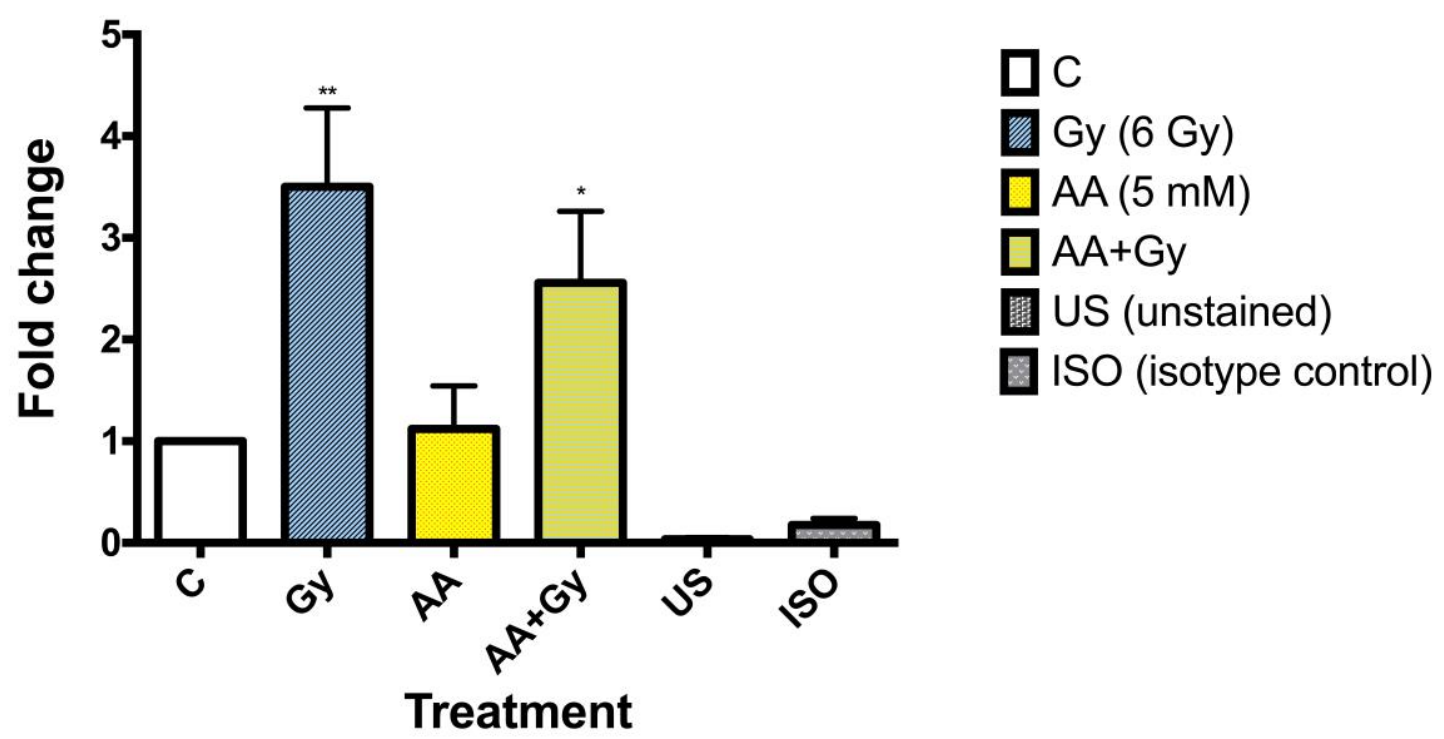

Figure 12. Flow cytometry derived percent positive $\mathrm{YH} 2 \mathrm{AX}$ staining in T98G cells

Flow cytometry derived percent positive $\mathrm{rH} 2 \mathrm{AX}$ staining (fold change + Standard Error of the Mean [SEM]) by treatment group for the T98G cell line. Graph includes data from 7 independent experiments, and each treatment group has a sample size of between 5 and 7 experiments. Irradiation and combination treatment groups were statistically significantly greater than control (P values: Gy 0.0048, AA 0.7380, AA+Gy 0.0232). Graph and statistics produced with Prism V6.0. 
$\gamma \mathrm{H} 2 \mathrm{AX}$ Median Fluorescence Intensity

T98G

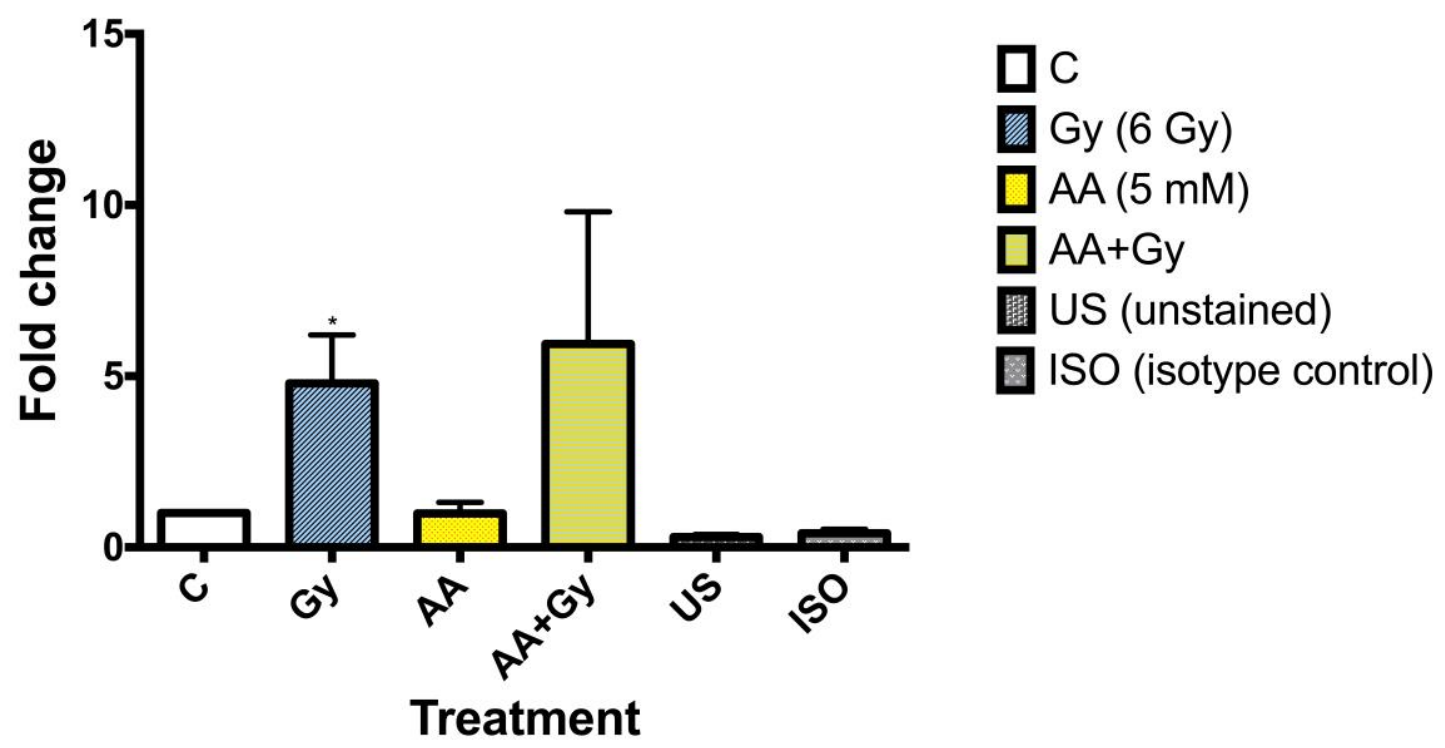

Figure 13. Flow cytometry derived median fluorescence intensity $\mathrm{YH2AX}$ staining in T98G cells

Flow cytometry derived median fluorescence intensity YH2AX staining (fold change + Standard Error of the Mean [SEM]) by treatment group for the T98G cell line. Graph includes data from 7 independent experiments, and each treatment group has a sample size of between 5 and 7 experiments. Irradiation was statistically significantly greater than control (P values: Gy 0.0139, AA 0.9662, AA+Gy 0.1527). Graph and statistics produced with Prism V6.0. 
1003 is another alternative GBM cell type used in this investigation. It differs from GL261 and T98G in that it is a human primary GBM cell developed from material from patients undergoing debulking surgery, rather than an immortalised cell line. Because it has undergone less passaging, and presumably genetic change, since the original in situ tumour, 1003 might be considered a closer representation of GBM in its native state. Although each individual tumour will have its own particular tumorigenic alterations (see 'Glioblastoma Multiforme').

Figure 14 displays results from the same experiment undertaken on the cell lines, as described in Figures 10-13, carried out on 1003 cells. 1003 cells followed a similar trend as the cell lines. Irradiation only gave rise to an approximately 2.5 fold increase in $\mathrm{YH} 2 \mathrm{AX}$ positive fluorescence, ascorbate caused a similar but more variable amplification in the marker, and combination treatment showed the greatest increase in an less than additive manner.

Finally, 1003 is also laid out with MFI analysis in Figure 15. In a similar fashion to the previous two cell types, MFI processed data from 1003 cells enhanced variation between treatment groups compared to percent positive analysis. Ascorbate treated cells fluoresced only slightly more than control cells, while irradiation caused many fold more $\mathrm{YH} 2 \mathrm{AX}$ signal per cell, an average of 7 times more fluorescence. Combination treatment resulted in the most $\mathrm{YH}_{2} \mathrm{AX}$ fluorescence per cell in an additive way, with a nearly 8 -fold mean increase, although this was not statistically significantly more than irradiation alone.

In conclusion, 3 GBM cell types with vastly different origins; a murine GBM model, an immortalised human GBM cell line, and a human primary cell derived directly from a patient, have been treated with the same doses of ascorbate and irradiation in the same experiments, and YH2AX fluorescence was measured with flow cytometry. Although each cell type has inevitable variation, overall trends are remarkably consistent. 
Specifically, this overall trend is that both ascorbate and irradiation increase the fluorescence of $\Upsilon \mathrm{H} 2 \mathrm{AX}$, irradiation more so than ascorbate, while cells that are treated with both present with $\mathrm{YH} 2 \mathrm{AX}$ stain higher than either treatment alone. 
$\gamma$ H2AX Percent Positive 1003

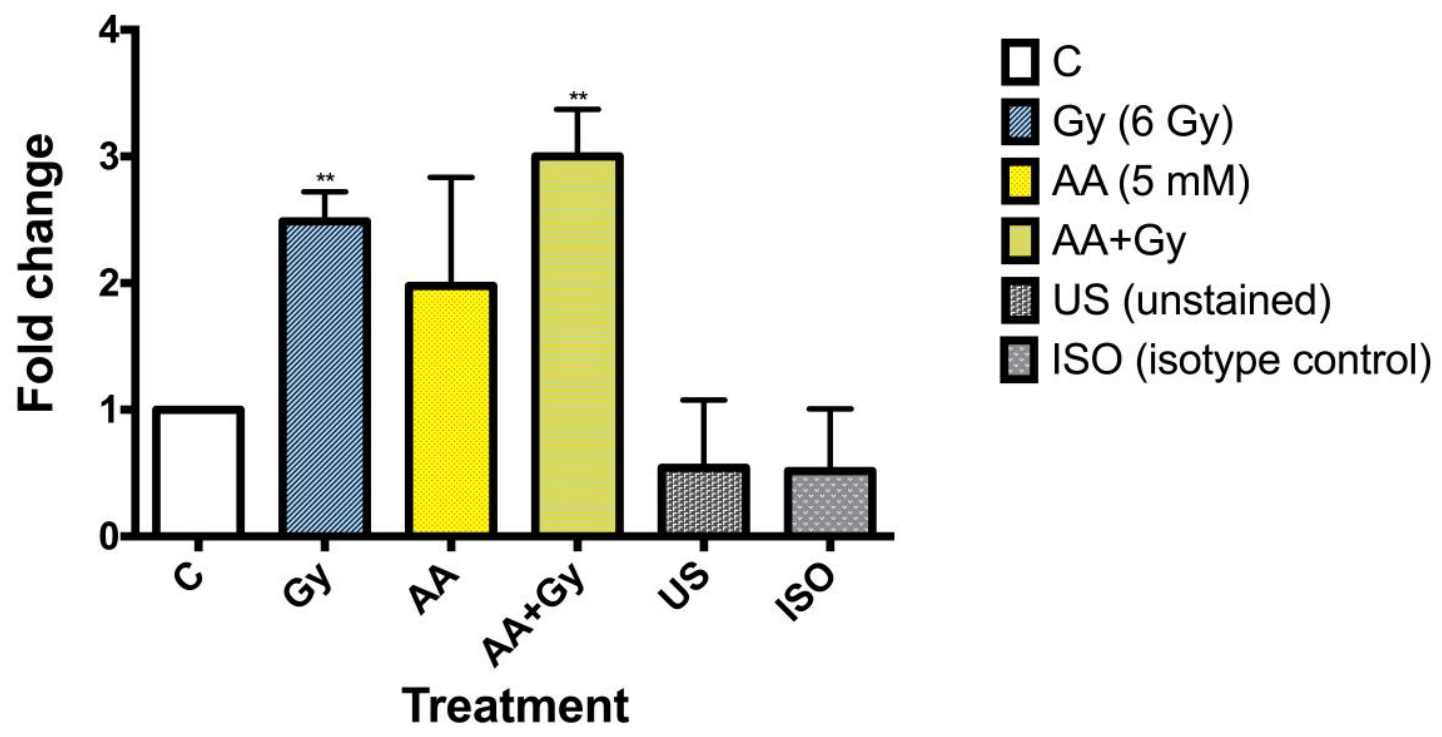

Figure 14. Flow cytometry derived percent positive $\mathrm{YH} 2 \mathrm{AX}$ staining in 1003 cells

Flow cytometry derived percent positive $\Upsilon$ H2AX staining (fold change + Standard Error of the Mean [SEM]) by treatment group for the 1003 cell line. Graph includes data from 3 independent experiments, and all treatment groups have a sample size of 3 experiments. Irradiation and combination treatment groups were statistically significantly greater than control (P values: Gy 0.0032, AA 0.3174, AA+Gy 0.0057). Graph and statistics produced with Prism V6.0. 
$\gamma \mathrm{H} 2 \mathrm{AX}$ Median Fluorescence Intensity

1003

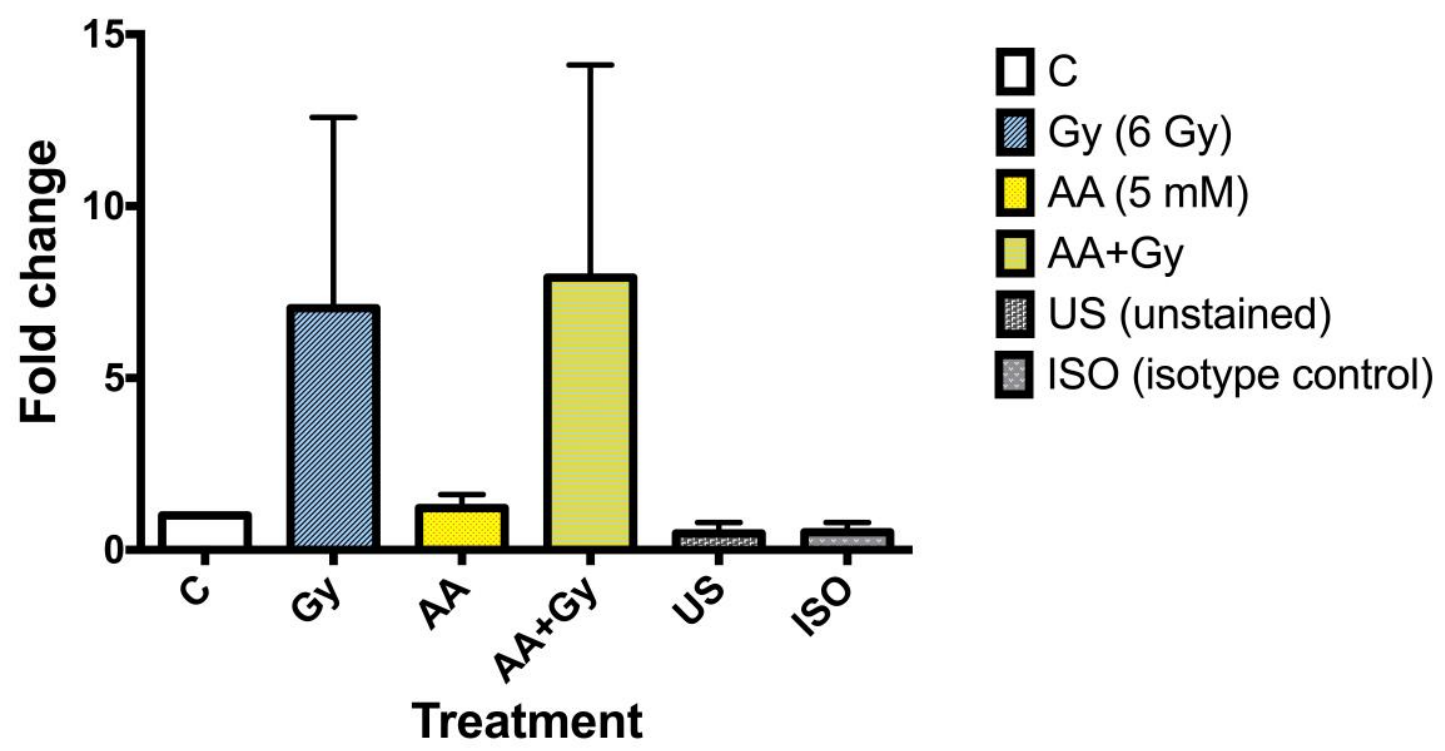

Figure 15. Flow cytometry derived median fluorescence intensity $\mathrm{CH}_{2} \mathrm{AX}$ staining in 1003 cells

Flow cytometry derived median fluorescence intensity YH2AX staining (fold change + Standard Error of the Mean [SEM]) by treatment group for the 1003 cell line. Graph includes data from 3 independent experiments, and all treatment groups have a sample size of 3 experiments. No treatment groups were statistically significantly greater than control (P values: Gy 0.3382, AA 0.6105, AA+Gy 0.3257). Graph and statistics produced with Prism V6.0. 


\section{8-hydroxy-2-deoxyguanosine}

8-hydroxy-2-deoxyguanosine (8-OHdG), like $\mathrm{YH} 2 \mathrm{AX}$, is an

indicator of DNA damage. It is a particular hydroxylation modification of the guanosine DNA nucleoside that forms in response to oxidative stress. In this investigation it was measured with flow cytometry alongside $\mathrm{rH} 2 \mathrm{AX}$, in order to compare the specific types of damage caused by ascorbate and irradiation individually and in combination. As an additional treatment group for this indicator, $500 \mu \mathrm{M}$ of hydrogen peroxide was included as a positive control, since hydrogen peroxide's mechanism of DNA damage is oxidative stress from reactive oxygen species (see 'DNA Damaging Effects of Ascorbate'). Data for 8-OHdG were generated and presented in the same way as the $\mathrm{rH} 2 \mathrm{AX}$ indicator, with the only difference being the use of a secondary antibody and the additional hydrogen peroxide group.

Figure 16 is the percent positive presentation fold change of 8$\mathrm{OHdG}$ fluorescence in response to the various treatment groups for the GL261 cell line. For this cell line, $\mathrm{H}_{2} \mathrm{O}_{2}$ response was surprisingly low, only around 1.5-fold greater than control untreated cells. Irradiation alone and combination treatments were approximately similar, reaching to around 2fold higher than control, while ascorbate only caused 3.5-fold greater 8OHdG signal.

The high ascorbate result is not greatly surprising, as the ascorbate DNA damaging effect will be caused by prooxidant species that cause the particular DNA damage 8-OHdG. However, the lower 8-OHdG result in response to combination treatments is dissimilar to that found with $\mathrm{YH} 2 \mathrm{AX}$. This interesting outcome appears to show irradiation decreased the number of cells with 8-OHdG due to ascorbate. Another interesting point to note is the difference between ascorbate and hydrogen peroxide. Hypothetically, 5 $\mathrm{mM}$ ascorbate should produce a similar degree of damage as $50 \mu \mathrm{M}$ hydrogen peroxide (Castro, McConnell et al. 2014), however this calibration was calculated with intracellular ROS load, and thus may not be directly applicable to the different marker. 
As an example of the need to analyse data from all perspectives, Figure 17 displays the same experiments as Figure 16, but showing median level of 8-OHdG per cell, instead of percent positive cells. In this case the trend is quite different. Although combination treatment has a smaller proportion of 8-OHdG positive cells than ascorbate alone, it appears that these cells have an overall greater fluorescence when viewed this way. Compared with irradiation only, combination treatment causes about the same proportion of cells to show DNA damage, but judging by overall MFI, cells that are damaged already become more damaged by with both treatments. Therefore, in this case combination treatment causes cells that are already damaged to become more so.

Another interesting aspect is the relatively higher $8-\mathrm{OHdG}$ signal per cell in response to hydrogen peroxide in MFI graphs as opposed to percent positive of the population. Instead of possessing only half the 8$\mathrm{OHdG}$ as ascorbate in percent positive display, indicating a less widespread induction of DNA damage, MFI shows hydrogen peroxide causing an even more substantial response in the subset of cells that are affected. 
8OHdG Percent Positive GL261

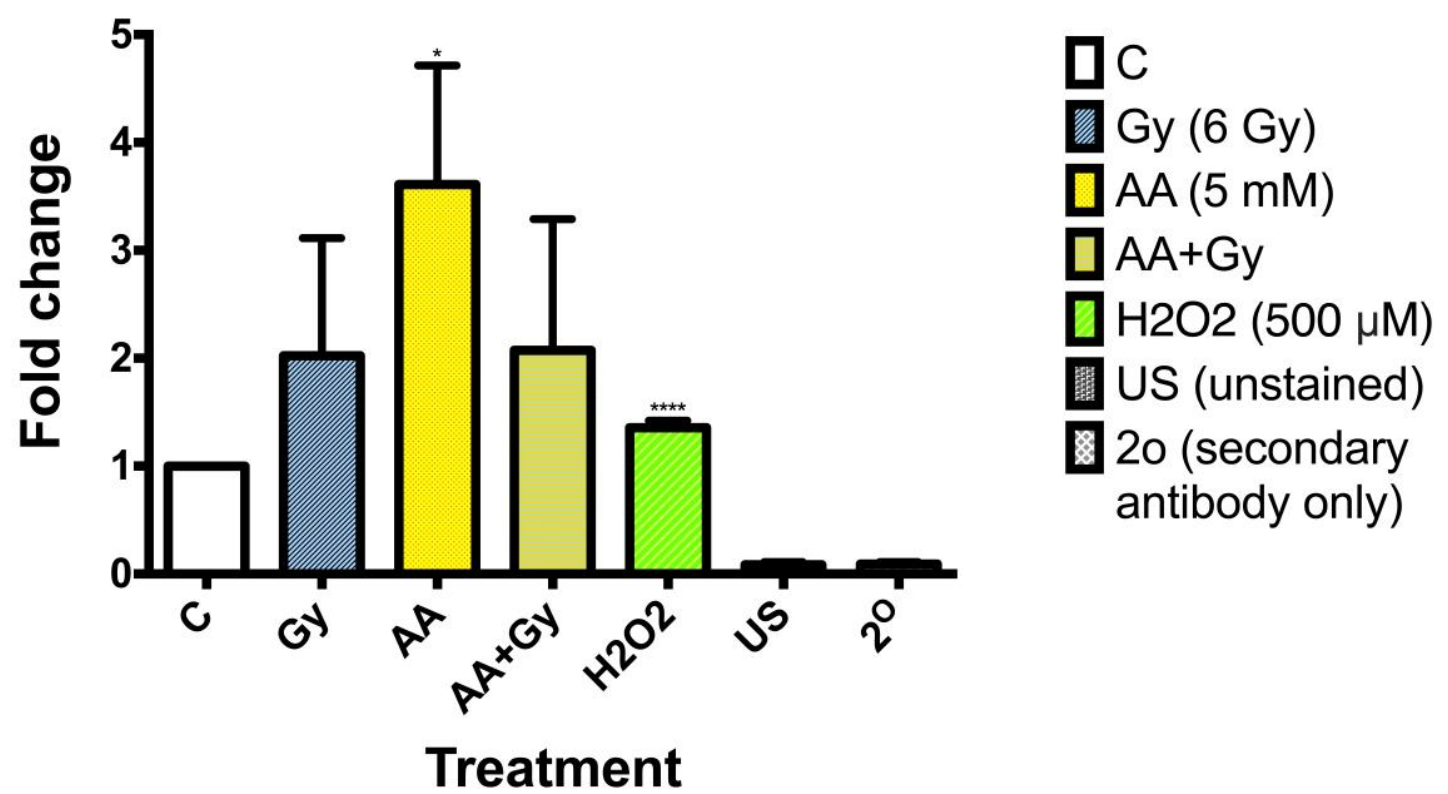

Figure 16. Flow cytometry derived percent positive 8-hydroxy-2-deoxyguanosine staining in GL261 cells

Flow cytometry derived percent positive 8-hydroxy-2-deoxyguanosine staining (fold change +

Standard Error of the Mean [SEM]) by treatment group for the GL261 cell line. Graph includes data from 10 independent experiments, and each treatment group has a sample size of between 4 and 10 experiments. Ascorbate and hydrogen peroxide treatment groups were statistically significantly greater than control (P values: Gy 0.1904, AA 0.0117, AA+Gy 0.2160, $\mathrm{H}_{2} \mathrm{O}_{2}<0.0001$ ). Graph and statistics produced with Prism V6.0. 
80HdG Median Fluorescence Intensity GL261

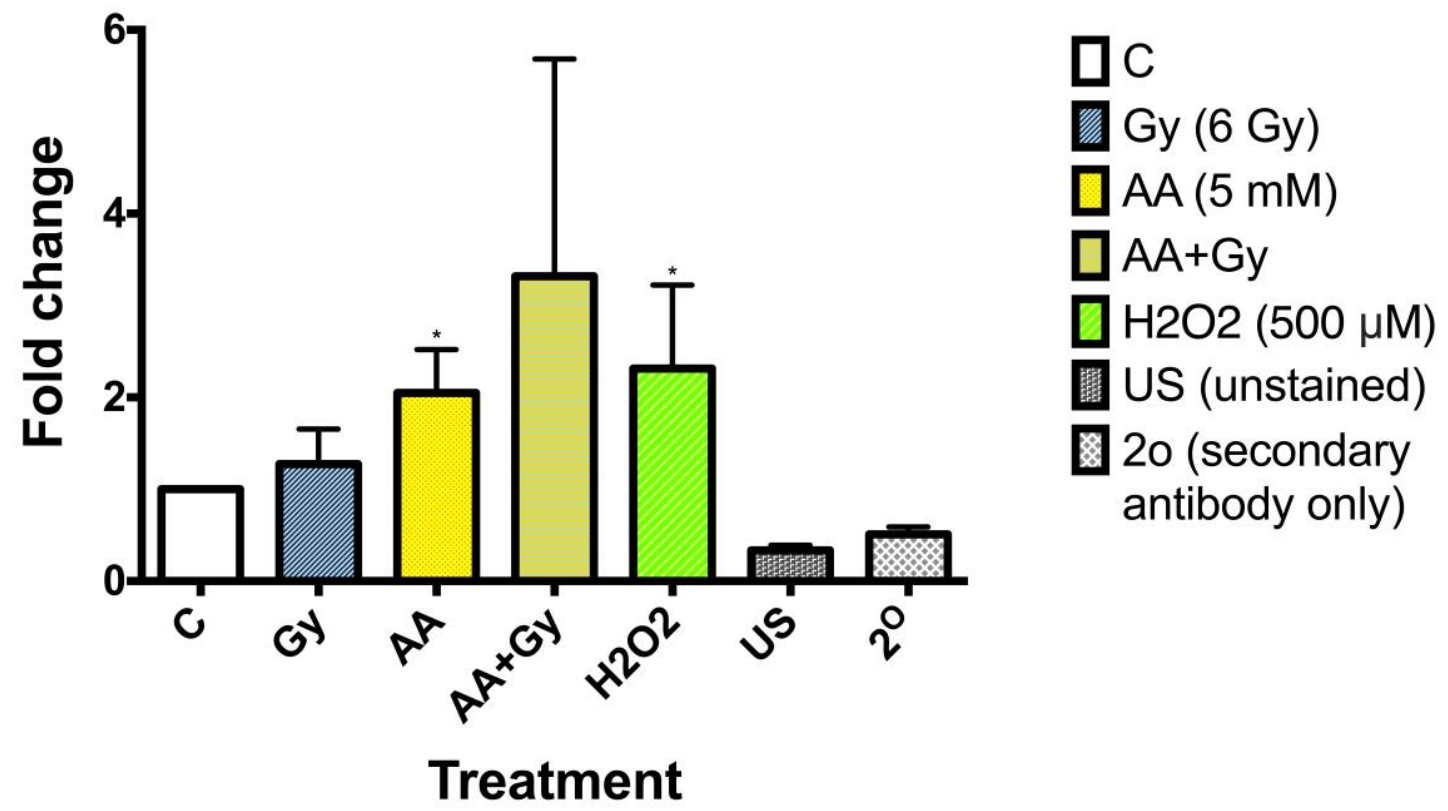

Figure 17. Flow cytometry derived median fluorescence intensity 8-hydroxy-2-deoxyguanosine staining in GL261 cells

Flow cytometry derived median fluorescence intensity 8-hydroxy-2-deoxyguanosine staining (fold change + Standard Error of the Mean [SEM]) by treatment group for the GL261 cell line. Graph includes data from 10 independent experiments, and each treatment group has a sample size of between 4 and 10 experiments. Ascorbate and hydrogen peroxide treatment groups were statistically significantly greater than control (P values: Gy 0.3119 , AA 0.0167 , AA+Gy $0.1723, \mathrm{H}_{2} \mathrm{O}_{2}$ 0.312). Graph and statistics produced with Prism V6.0. 


\section{OHdG Percent Positive T98G}

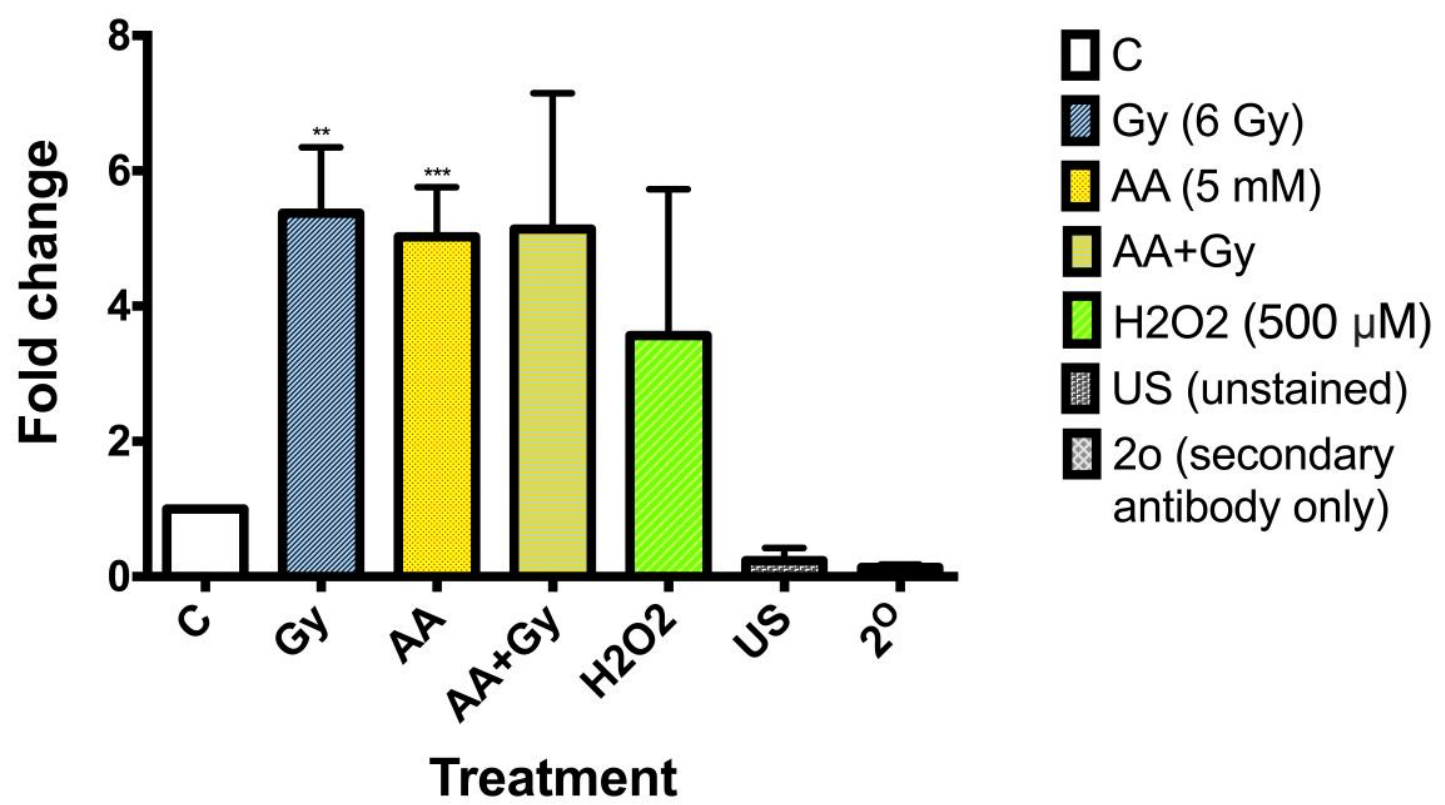

Figure 18. Flow cytometry derived percent positive 8-hydroxy-2-deoxyguanosine staining in T98G cells

Flow cytometry derived percent positive 8-hydroxy-2-deoxyguanosine staining (fold change + Standard Error of the Mean [SEM]) by treatment group for the T98G cell line. Graph includes data from 5 independent experiments, and each treatment group has a sample size of between 3 and 5 experiments. Irradiation and ascorbate treatment groups were statistically significantly greater than control (P values: Gy 0.0021, AA 0.0006, AA+Gy 0.0515, $\mathrm{H}_{2} \mathrm{O}_{2}$ 0.1551). Graph and statistics produced with Prism V6.0. 
80HdG Median Fluorescence Intensity

T98G

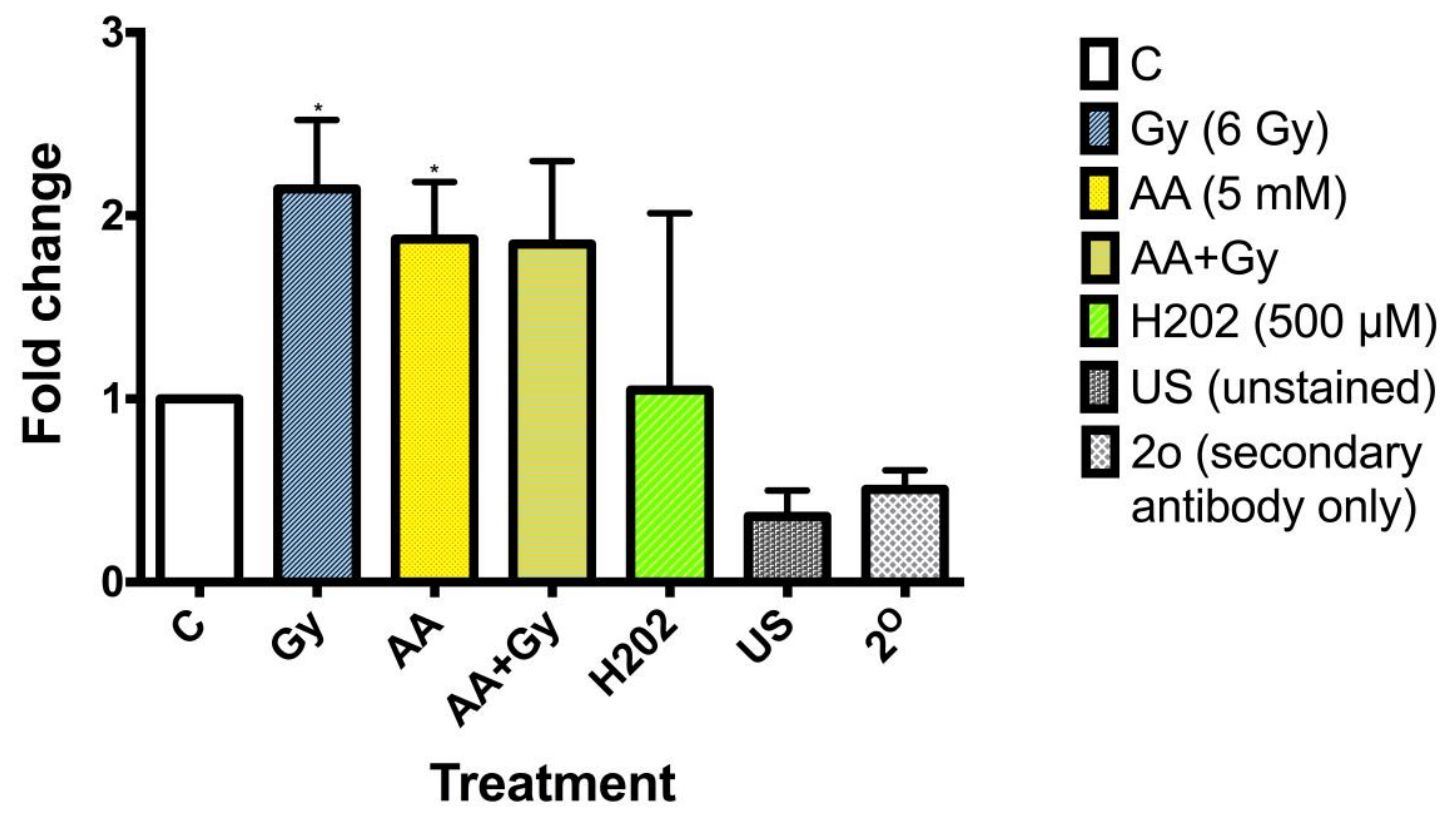

Figure 19. Flow cytometry derived median fluorescence intensity 8-hydroxy-2-deoxyguanosine staining in T98G cells

Flow cytometry derived median fluorescence intensity 8-hydroxy-2-deoxyguanosine staining (fold change + Standard Error of the Mean [SEM]) by treatment group for the T98G cell line. Graph includes data from 5 independent experiments, and each treatment group has a sample size of between 3 and 5 experiments. Irradiation and ascorbate treatment groups were statistically significantly greater than control (P values: Gy 0.0161, AA 0.0234, AA+Gy 0.0710, $\mathrm{H}_{2} \mathrm{O}_{2} 0.9103$ ). Graph and statistics produced with Prism V6.0. 
Figure 18 set outs the of number $8-\mathrm{OHdG}$ positive cells in response to treatment in the $\mathrm{T} 98 \mathrm{G}$ cell line. In this particular case, treatments are remarkably alike in their fluorescence as irradiation, ascorbate, and combination treatments all increase $8-\mathrm{OHdG}$ around 5-fold over control cells. Hydrogen peroxide is the anomaly, increasing the proportion of cells with 8-OHdG damage only 3 -fold.

In Figure 19, the median level of 8-OHdG in each population is graphed for T98G cells, and again shows similar effects to that seen with percent positive. With MFI scoring, irradiation only treatment has higher 8OHdG than all other, including combination treatments, compared to percent positive. All treatments except hydrogen peroxide increase 8-OHdG per cell approximately 2 -fold. Similar to percent positive, hydrogen peroxide provokes the least increase in $8-\mathrm{OHdG}$ per cell, at only 1.1-fold greater than control.

Finally, 8-OHdG response to treatment was investigated in 1003 cells (Figure 20). This resulted in quite a different effect from the other two cells. For instance, irradiation alone caused a large effect, approximately 7.5-fold increase over control cells. Hydrogen peroxide caused a slightly larger increase than irradiation only. The high level of increase in the proportion of 8-OHdG cells with hydrogen peroxide indicates perhaps that $1003 \mathrm{~s}$ are not quite as resilient to hydrogen peroxide mediated-oxidative stress as the other two cell lines that produced a lower 8-OHdG signal. Also dissimilar to GL261 and T98G, 1003 8-OHdG staining showed that ascorbate and combination treatments have much lower effect than irradiation alone, around 2 and 5-fold respectively. This is unexpected, as ascorbate is thought to mediate DNA damage by reactive oxygen species, which would be predicted to cause a high level of oxidative damage and so 8-OHdG modifications. Yet, relative to other cell lines, ascorbate only treatment for 8-OHdG in 1003 cells has a comparable fold change response, at 2.3-fold, to GL261 percent positive (Figure 10). Combination treatment fold change, around 4.7-fold is similar to that seen in T98G 8-OHdG combination treatment. 
Figure 21 presents MFI analysis for the same data. The overall trend is much the same, only with irradiation treatment causing a greater increase in 8-OHdG signal than hydrogen peroxide treatment. Again, ascorbate only barely increased the amount of $8-\mathrm{OHdG}$ in cells, while the combination treatment increased it not quite 2 -fold. 


\section{OHdG Percent Positive 1003}

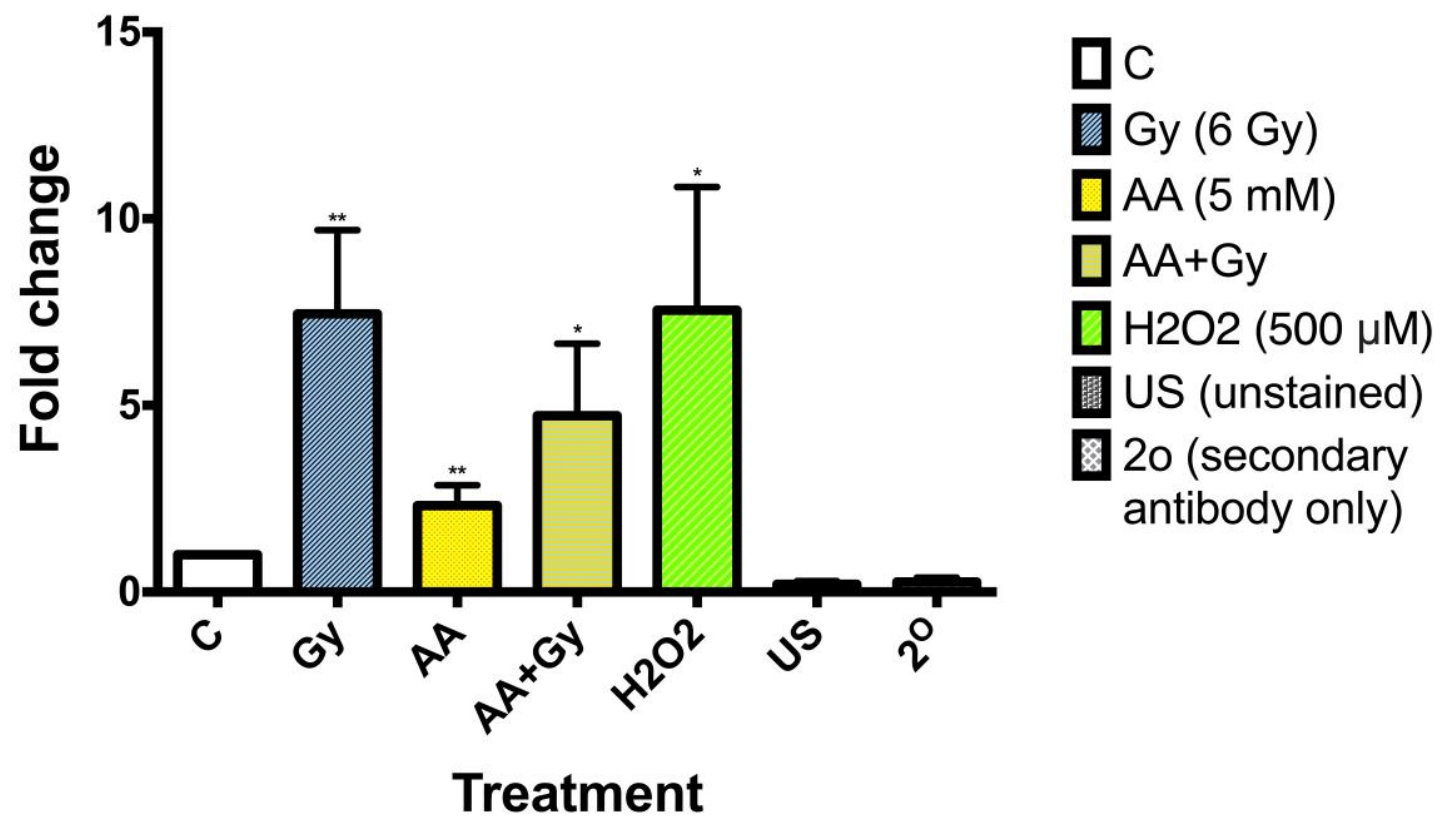

Figure 20. Flow cytometry derived percent positive 8-hydroxy-2-deoxyguanosine staining in 1003 cells

Flow cytometry derived percent positive 8-hydroxy-2-deoxyguanosine staining (fold change + Standard Error of the Mean [SEM]) by treatment group for the 1003 cell line. Graph includes data from 12 independent experiments, and each treatment group has a sample size of between 7 and 12 experiments. All treatment groups were statistically significantly greater than control (P values: Gy 0.0034, AA 0.0076, AA+Gy 0.0364, $\mathrm{H}_{2} \mathrm{O}_{2}$ 0.167). Graph and statistics produced with Prism V6.0. 
8OHdG Median Fluorescence Intensity

1003

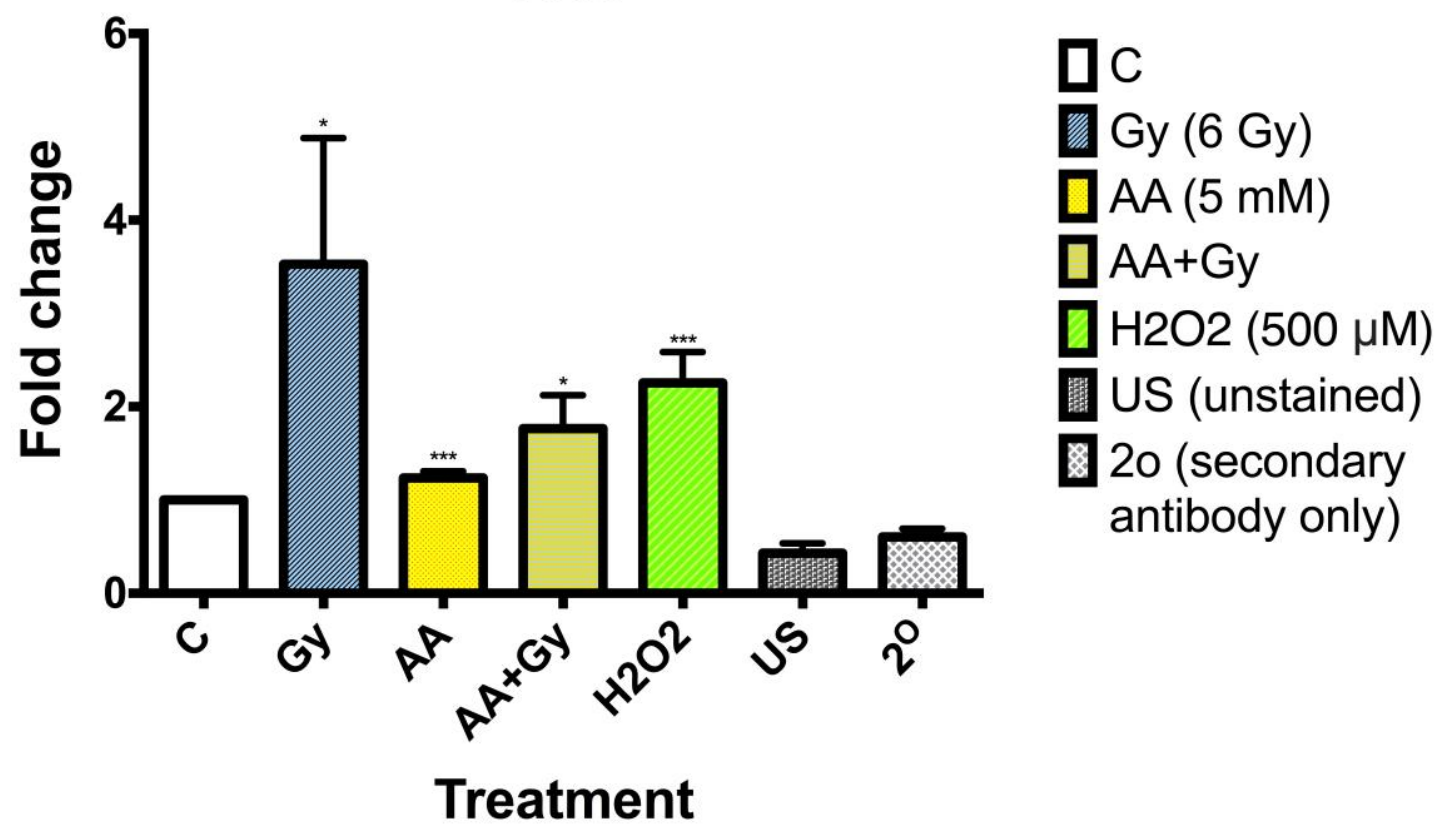

Figure 21. Flow cytometry derived median fluorescence intensity 8-hydroxy-2-deoxyguanosine staining in 1003 cells

Flow cytometry derived median fluorescence intensity 8-hydroxy-2-deoxyguanosine staining (fold change + Standard Error of the Mean [SEM]) by treatment group for the 1003 cell line. Graph includes data from 12 independent experiments, and each treatment group has a sample size of between 7 and 12 experiments. All treatment groups were statistically significantly greater than control (P values: Gy 0.423, AA 0.0007, AA+Gy 0.0216, $\mathrm{H}_{2} \mathrm{O}_{2}$ 0.0002). Graph and statistics produced with Prism V6.0. 


\section{Single Cell Gel Electrophoresis/Comet Tail Assay}

The single cell gel electrophoresis assay, also known as the comet tail assay, was established as an additional assay to investigate ascorbate's induction of DSBs, and so confirm ascorbate's radiosensitising role in vitro, the first objective of the project.

Cells were harvested and treated with the same dose of ascorbate or irradiation as in the flow cytometry assay, before being embedded in low melting point agarose, lysed, and electrophoresed. The DNA was stained with PI and imaged to assess relative DSB DNA damage. This particular variant of the comet tail assay is designed to detect DSBs only, as it is carried out under neutral conditions and so should not incorporate other types of damages, such as single stranded breaks (SSBs).

\section{Analysis}

As described in 'Methods', cell nuclei/ 'comets' were analysed with a ImageJ plugin designed for analysis of the comet tail assay. Figure 22 displays an example analysis of one typical cell nuclei and the graphical output from ImageJ.

Figure 23 is an example of cell nuclei response to treatment. This figure shows typical comet tails produced from either untreated control cells (on the left) compared with cells that were treated with 80 Gy of irradiation. As can be seen on the left, even untreated cells have a basal level of DSBs, represented by small tails emanating from some cell nuclei. Cells treated with $80 \mathrm{~Gy}$, the highest dose used in this investigation, produce comet tails that are severely fragmented, due to the large proportion of DSBs caused by this high dose.

Because this assay had not been established in the lab, two experiments were carried out in order to optimise and validate the process; an ascorbate time course and irradiation dose response. 

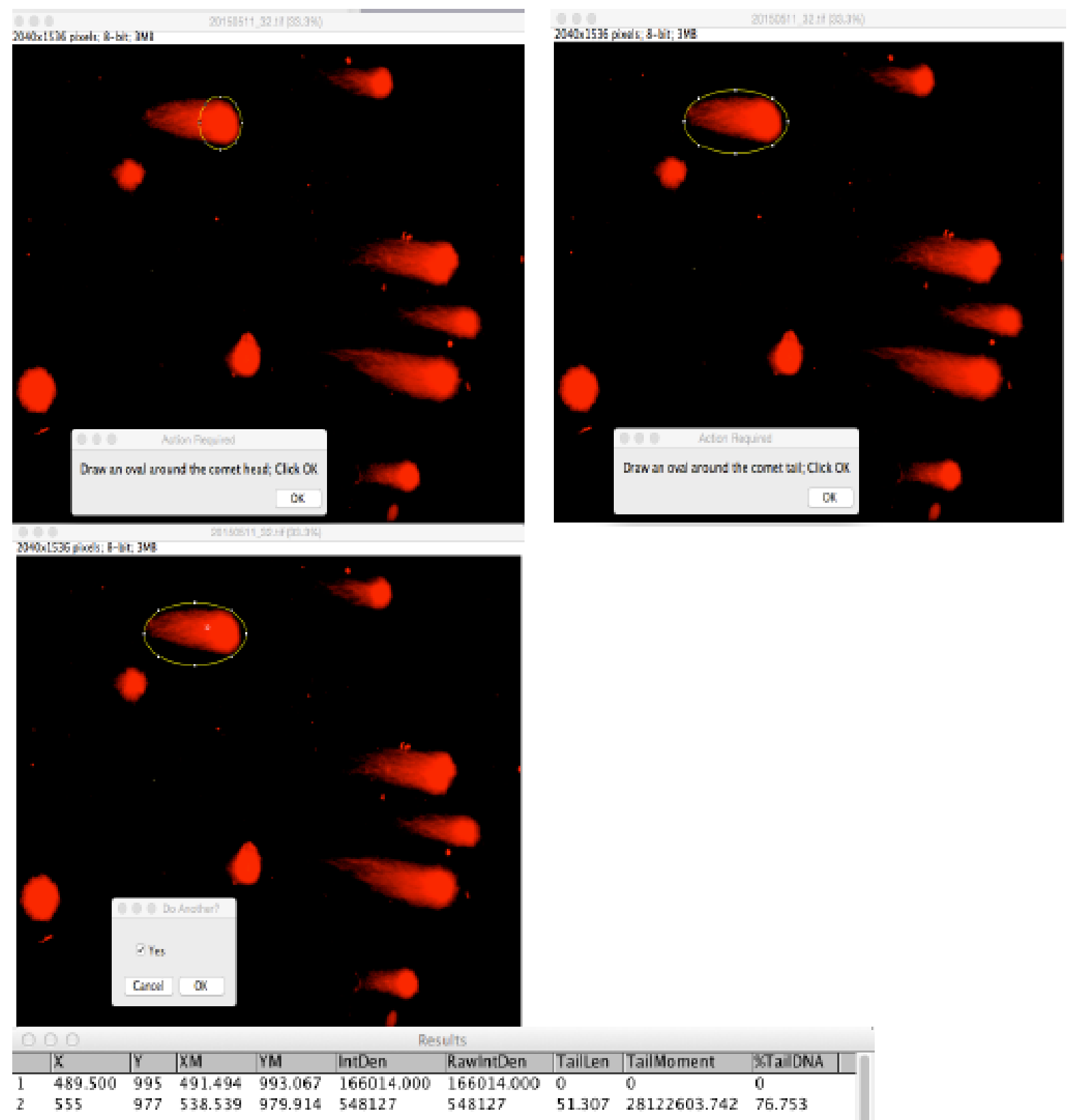

Figure 22. Graphical representative analysis of comet tails using the ImageJ comet tail plugin

A. An oval is drawn around the comet tail head. B. The oval is extended to encircle the comet including the tail. C. The measurements are confirmed and a numbered label is added to the comet image. D. Data is displayed in an ImageJ window before export to Excel. 

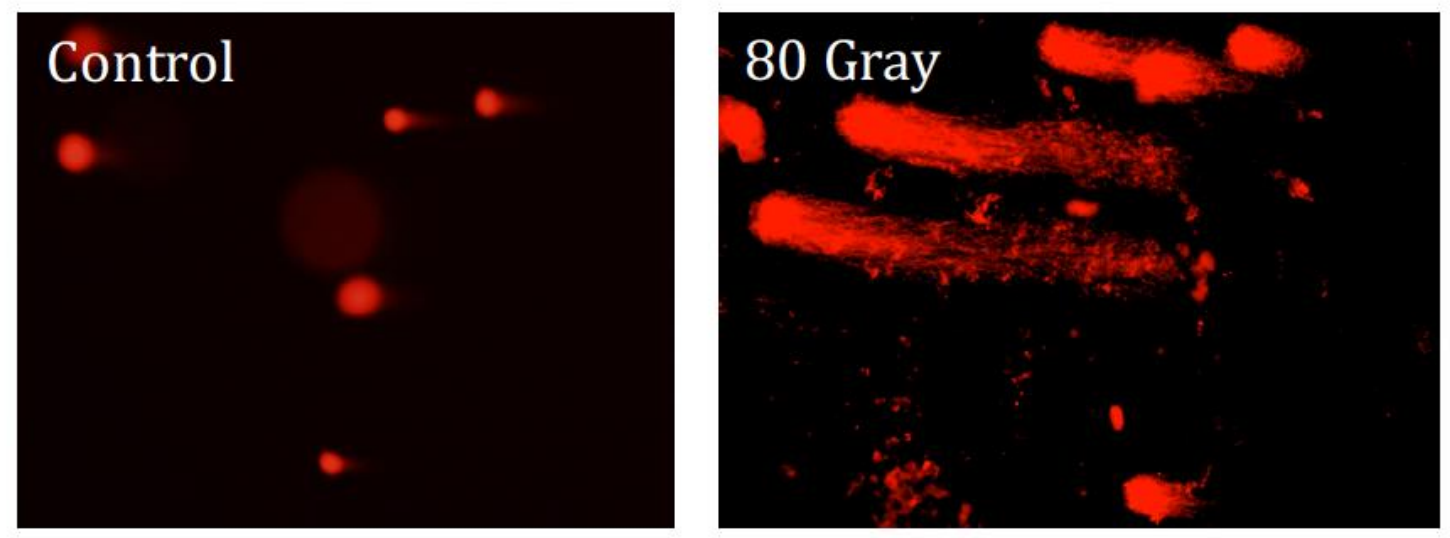

Figure 23. Example display of typical comet nuclei in response to either no treatment $(\mathrm{L})$ or 80 Gy of irradiation (R) 


\section{Ascorbate Time Course}

From $\mathrm{YH} 2 \mathrm{AX}$ experiments, it was expected that ascorbate would cause an increase in DSBs, or at least general DNA damage. An ascorbate time course was an opportunity to confirm ascorbate was causing DSBs in particular and determine whether this increased over time, as well as investigate a general rate of repair.

Figure 24 exhibits the results of this ascorbate time course in GL261 cells, using the comet tail assay parameter that was found to be the most reproducible, tail length. The aim of the experiment was to try and pinpoint from what time ascorbate begins to cause DSB damage, what is the rate of increase in DSBs over time in response to ascorbate, and how long is required without the presence of ascorbate to repair DSBs created.

To the left of the partition are time points for how long GL261s were exposed to ascorbate in media, between 15 minutes and 3 hours. Note each of these times describes the length cells were incubated in ascorbate at the $5 \mathrm{mM}$ concentration before being diluted in agarose. Thus the total amount of time each group was exposed to any ascorbate was the time point label plus 30 minutes required to allow drying of agarose before lysis. Ascorbate causes a clear increase in DSBs over time, such that even at 15 minutes, tail length is nearly 4-fold longer than control untreated cells, and at the longest time point, 3-hour treatment, ascorbate resulted in a 7-fold increase in tail length. However, these DSBs are fairly rapidly repaired.

To the right of the partition are two time points that outline the swift repair of DSBs by GL261s, at least for cells that do not die outright. For these time points, cells were treated for one hour with ascorbate, before being allowed to recover in ascorbate free media for the indicated time, minus treatment time. Either 7 or 23 hours post ascorbate treatment, cells were harvested and processed in the same manner as other time points. Both 8 hours and 24 hours after initialisation of ascorbate treatment, tail length fold change was approaching 1 relative to control, indicating nearly all DSBs had been repaired before this time. However, because media was 
aspirated during the harvesting process, cells that had died and were in suspension would not have been included.

Figure 25 represents data from the same ascorbate time course, yet with another comet tail parameter, tail moment. Tail Moment is the length of the tail times the integrated density of the tail (see 'Methods: Comet tail assay/Single cell gel electrophoresis'), thus is a measure that combines how long the comet tail is, as well as the extent of DNA that has been electrophoresed into the tail. Tail moment analysis of the data gives a not as clear trend as tail length. In the treatment time points, 15 minutes is low, while each other time point appears roughly similar, apart from the clear anomaly of a large tail moment average in the 1.5-hour time point. As with tail length, tail moment displays almost complete repair of DSBs within 7 hours of recovery time.

The percentage of the DNA in the tail is another parameter output produced by the comet tail plugin. Figure 26 displays the results of the ascorbate time course percent tail DNA measures. Interestingly, percentage tail DNA varied only very little in all the comet tail assay experiments undertaken for this investigation. This may be related to the particulars of the comet tail assay plugin, which, for this parameter, limits the amount of variation possible between each treatment group by expressing values as percentage values between 50 and 100 .

In this analysis, plugin measurement allowed either the comet head only, or the entire comet (see Figure 22). Therefore, the output, although correlated with the true percentage of DNA in the tail, was not strictly a measure of percent tail DNA. 


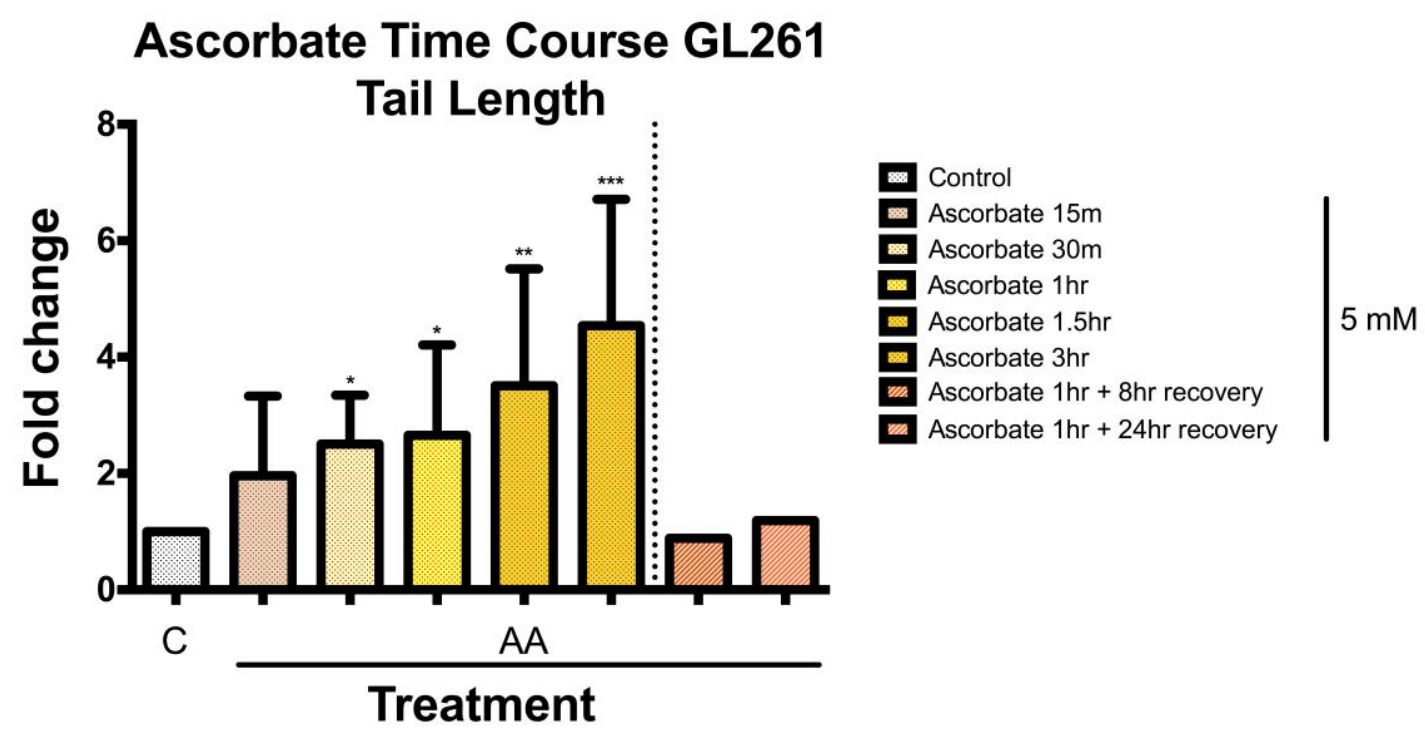

Figure 24. Single cell gel electrophoresis ascorbate time course tail lengths

Single cell gel electrophoresis ascorbate time course tail lengths (fold change + Standard Error of the Mean [SEM]) with GL261 cells. In the treatment groups to the left of the dotted line, cells were exposed to $5 \mathrm{mM}$ of ascorbate for the time indicated before combination with agarose and application onto pre-agarose coated slides. Cell-containing agarose was allowed to dry for 30 minutes on slides before lysis. In the treatment groups to the right of the dotted line, cells were allowed to recover in un-ascorbate exposed media. Cells were exposed to ascorbate for a set 30 minutes before incubation in fresh media. Cells were harvested, combined with agarose and lysed at the given time post initiation of treatment. Graph contains data from 11 independent experiments, and each time point has a sample size of between 1 and 7 experiments. All time points except for Ascorbate $15 \mathrm{~m}, 1 \mathrm{hr}+8 \mathrm{hr}$ recovery, and $1 \mathrm{hr}+24 \mathrm{hr}$ recovery were statistically significantly higher than control (P values: 15m 0.0552, 30m 0.0361, 1hr 0.0338, 1.5hr 0.0029, 3hr 0.0004, $1 \mathrm{hr}+8 \mathrm{hr}$ recovery and $1 \mathrm{hr}+24 \mathrm{hr}$ recovery $\mathrm{n} / \mathrm{a}$. Graph and statistics produced with Prism V6.0. 


\section{Ascorbate Time Course GL261}

\section{Tail Moment}

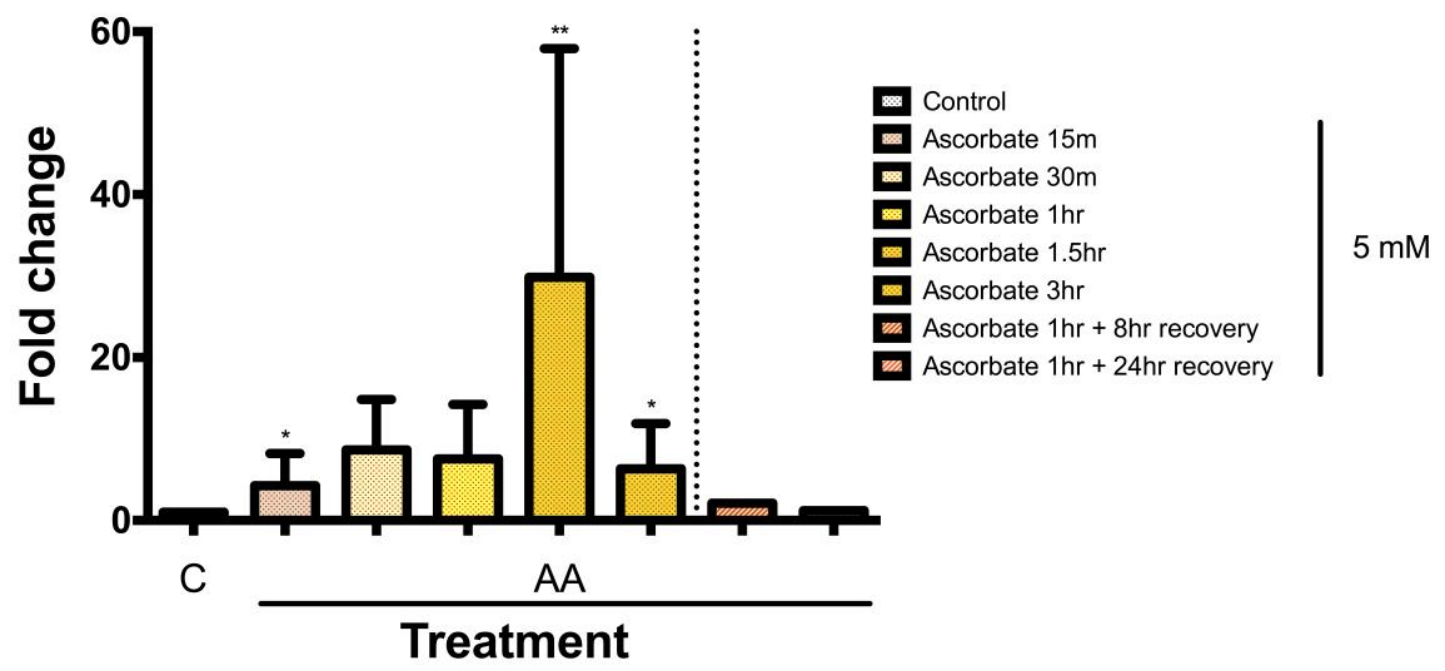

Figure 25. Single cell gel electrophoresis ascorbate time course tail moments

Single cell gel electrophoresis ascorbate time course tail moments (fold change + Standard Error of the Mean [SEM]) with GL261 cells. Cells were analysed as described in Figure 24. Ascorbate 15m, $1.5 \mathrm{hr}$, and $3 \mathrm{hr}$ were statistically significantly higher than control (P values: $15 \mathrm{~m} 0.0282,30 \mathrm{~m} 0.1331$, $1 \mathrm{hr} 0.0550,1.5 \mathrm{hr} 0.0095,3 \mathrm{hr} 0.0140,1 \mathrm{hr}+8 \mathrm{hr}$ recovery and $1 \mathrm{hr}+24 \mathrm{hr}$ recovery n/a. Graph and statistics produced with Prism V6.0. 


\section{Ascorbate Time Course GL261 Percent Tail DNA}

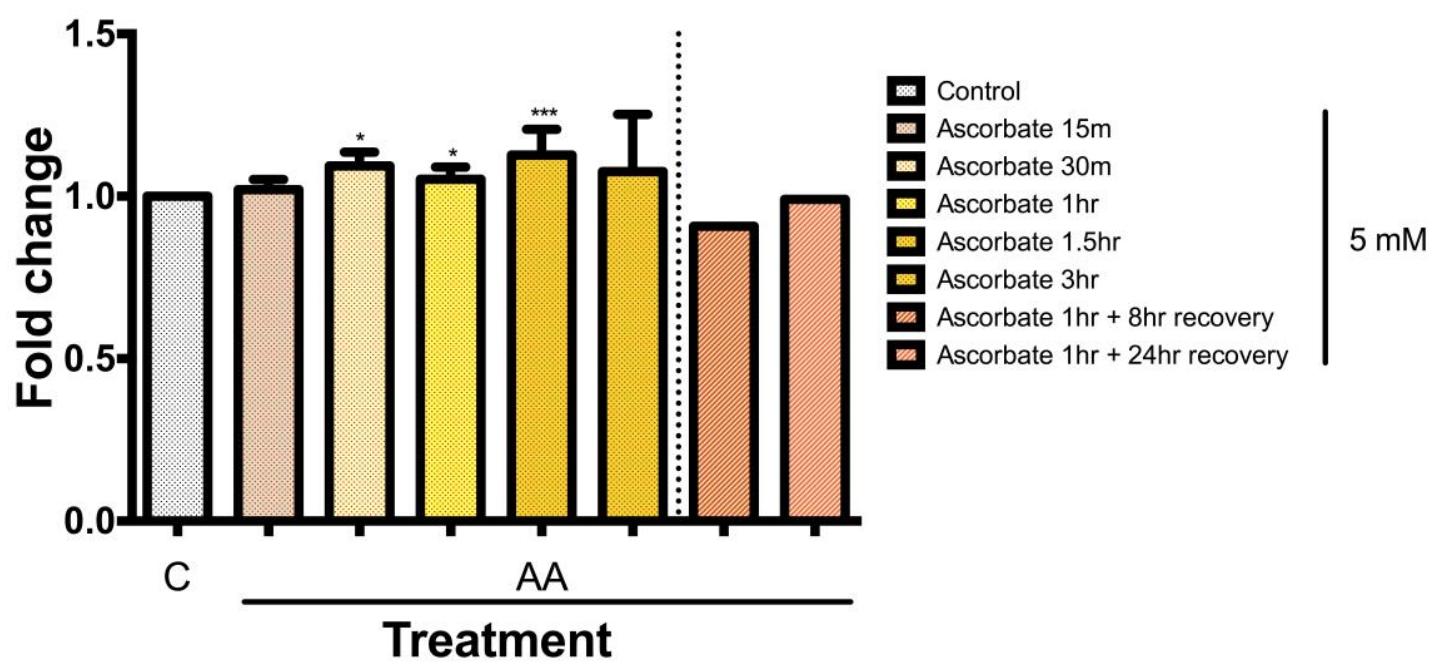

Figure 26. Single cell gel electrophoresis ascorbate time course percent tail DNA

Single cell gel electrophoresis ascorbate time course tail moments (fold change + Standard Error of the Mean [SEM]) with GL261 cells. Cells were analysed as described in Figure 24. Ascorbate 30m,

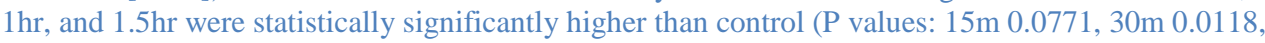
$1 \mathrm{hr} 0.0103,1.5 \mathrm{hr} 0.0005,3 \mathrm{hr} 0.2151,1 \mathrm{hr}+8 \mathrm{hr}$ recovery and $1 \mathrm{hr}+24 \mathrm{hr}$ recovery n/a. Graph and statistics produced with Prism V6.0. 
Whereas true percent tail DNA is calculated as described in the methods section:

$\%$ Tail DNA = Int. Density of Tail

Int. Density of Tail + Int. Density of Head

The comet tail plugin calculated a related but not identical:

$\%$ Tail DNA= Int. Density of Head - Int. Density Total

Int. Density of Head + Int. Density Total

As mentioned in methods, this formula results in a range of $50 \%$ to near $100 \%$, although most commonly comets were measured as $\sim 50-60 \%$ tail DNA.

\section{Irradiation Dose Response}

The second optimisation and validation experiment was a radiation dose response. Doses were chosen starting from the standard 6 Gy used in flow cytometry experiments, up until the large dose of $80 \mathrm{~Gy}$.

Figure 27 sets out the fold change increase in tail length caused by the various doses of irradiation. It can be seen that even the lowest dose of 6 Gy caused an increase in comet tail length, around 3-fold longer on average than control untreated cells. The increasing doses increased the tail length in a reproducible and predictable manner, and the largest irradiation dose, $80 \mathrm{~Gy}$, caused a fold change increase of approximately 7-fold greater than control. This supports increasing doses of irradiation causing a dosedependent increase in the number of DSBs.

Figure 28 represents the tail moment from the same experiments. Tail moment is the integrated density of the comet tail times the length of the tail. For the lower doses, it broadly follows the trend of increasing DSBs in response to increasing amounts of irradiation, although at higher doses the tail moment reaches a plateau, and even decreases. There are at least two explanations for this: either tail moment's inherent higher 
variability means that the final two doses, which were only analysed in one and two experiments respectively, need more replicates to increase and continue the trend; or, at higher doses of irradiation the integrated tail density does reach a plateau, and, because tail moment incorporates tail density into that parameter, tail moment plateaus also.

Percentage tail DNA for the irradiation dose response experiment is presented in Figure 29. In a similar way to the percent tail DNA in the ascorbate time course (Figure 26), this measure does not vary enough to be interpretable. 


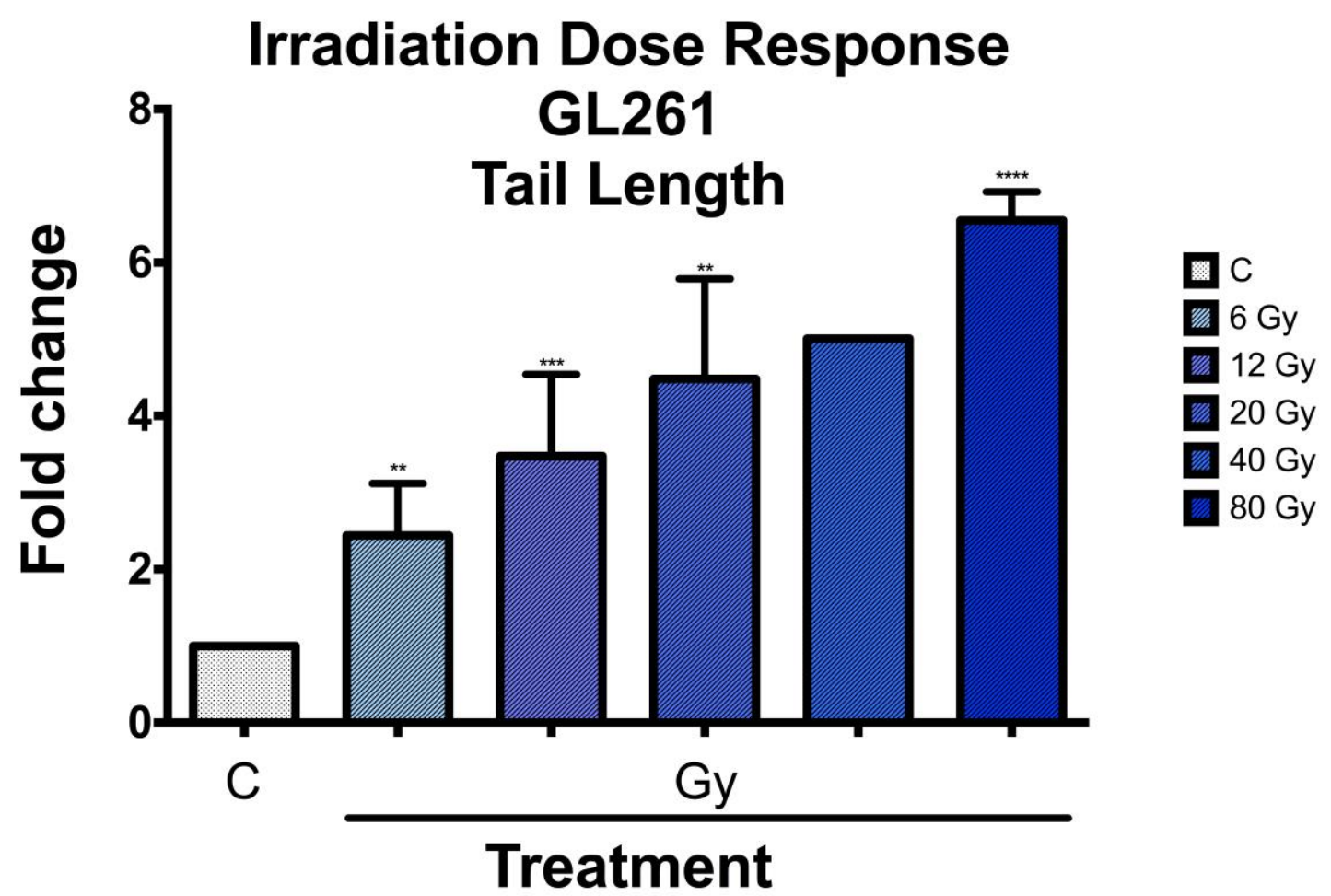

Figure 27. Single cell gel electrophoresis irradiation dose response tail lengths

Single cell gel electrophoresis irradiation dose response tail lengths (fold change + Standard Error of the Mean [SEM]) with GL261 cells. Cells were exposed to a given dose of irradiation before combination with agarose and application onto pre-agarose coated slides. Cell-containing agarose was allowed to dry for 30 minutes on slides before lysis. Graph contains data from 10 independent experiments, and each time point has a sample size of between 1 and 10 experiments. All irradiation doses except 40 Gy were statistically significantly higher than control (P values: 6 Gy 0.0081, 12 Gy $0.0006,20 \mathrm{~Gy} \mathrm{0.0033,} 40 \mathrm{~Gy}$ n/a, and $80 \mathrm{~Gy}<0.0001$. Graph and statistics produced with Prism V6.0. 


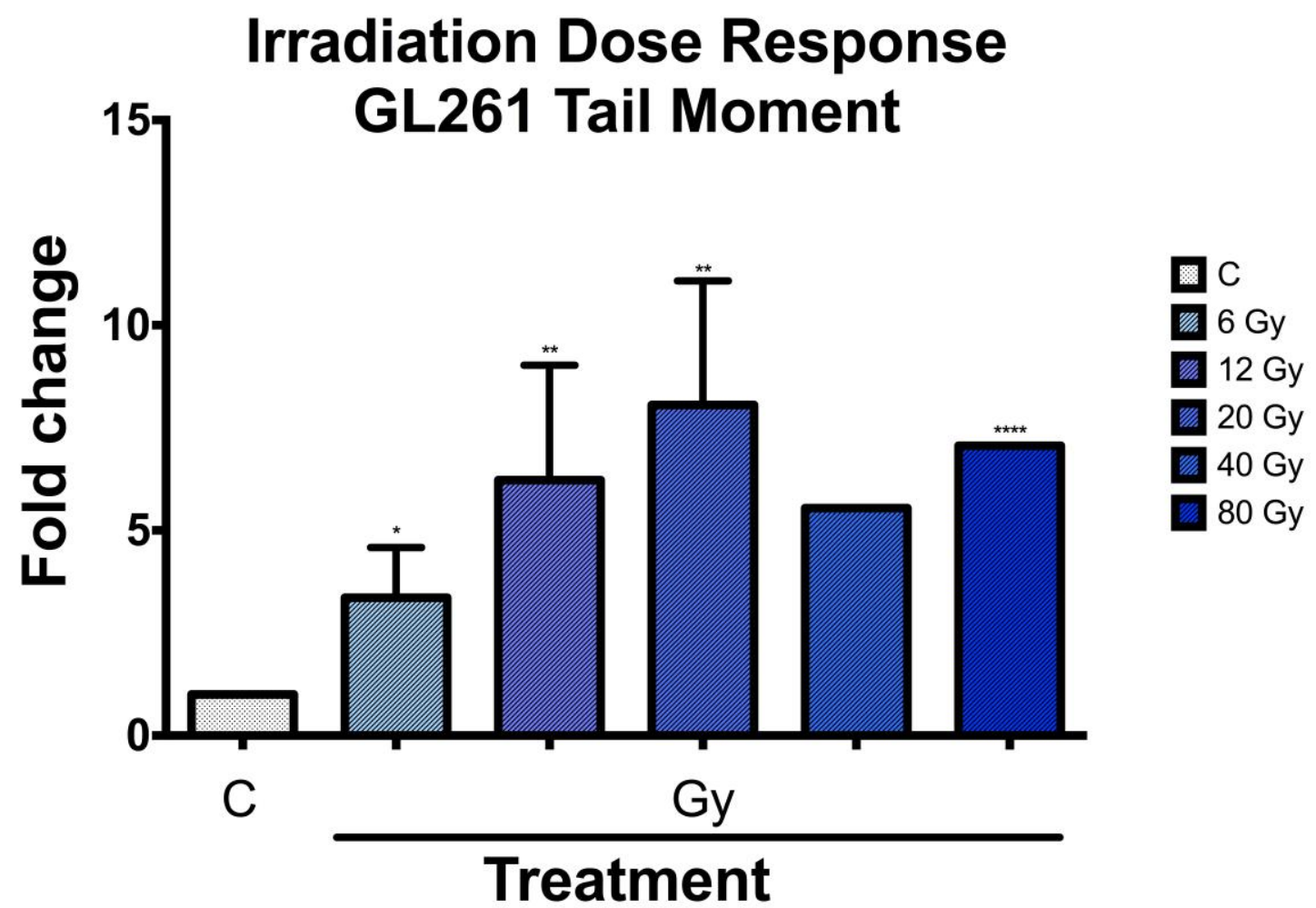

Figure 28. Single cell gel electrophoresis irradiation dose response tail moments.

Single cell gel electrophoresis irradiation dose response tail moments (fold change + Standard Error of the Mean [SEM]) with GL261 cells. Cells were treated as described in Figure 27. Graph contains data from 10 independent experiments, and each time point has a sample size of between 1 and 10 experiments. All irradiation doses except 40 Gy were statistically significantly higher than control (P values: 6 Gy 0.0139, 12 Gy 0.0028, 20 Gy 0.0081, 40 Gy n/a, and 80 Gy < 0.0001. Graph and statistics produced with Prism V6.0. 


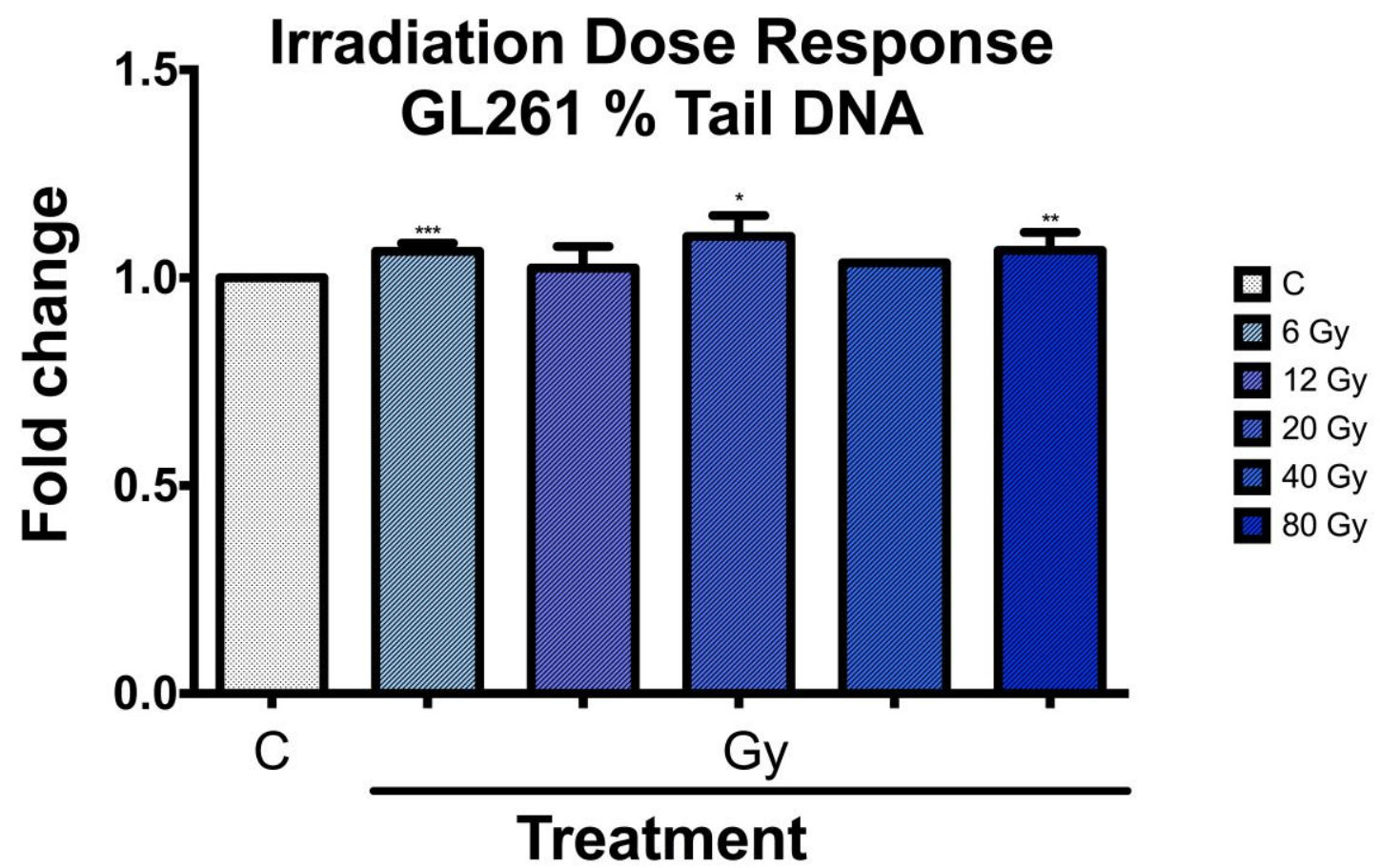

Figure 29. Single cell gel electrophoresis irradiation dose response percent tail DNA

Single cell gel electrophoresis irradiation dose response percent tail DNA (fold change + Standard Error of the Mean [SEM]) with GL261 cells. Cells were treated as described in Figure 27. Graph contains data from 10 independent experiments, and each time point has a sample size of between 1 and 10 experiments. All irradiation doses except $40 \mathrm{~Gy}$ were statistically significantly higher than control (P values: 6 Gy 0.0003, 12 Gy 0.3796, 20 Gy 0.0207, 40 Gy n/a, and 80 Gy 0.0016. Graph and statistics produced with Prism V6.0. 


\section{Combination Treatments}

The single cell gel electrophoresis, or comet tail assay, was also used to assess the effect of ascorbate on irradiation treatment in vitro. In the same manner as the flow cytometry experiment, cells were treated with 6 Gy irradiation, $5 \mathrm{mM}$ ascorbate and both treatments in combination. For this assay, an additional control was included, treatment of cells with ascorbate in PBS instead of media. This negative control was designed to assess the importance of transition metals present in the media, particularly to determine whether ascorbate could cause DSBs in the absence of metal catalysts.

Figure 30 displays the results of the combination treatments assessed with the tail length variable, with averages derived from 9 independent experiments. As expected both irradiation and ascorbate alone caused an increase in DSBs, irradiation increasing DSBs 2.1-fold over the control basal rate, and ascorbate increasing DSBs slightly more at 2.3-fold over untreated cells. The increases are presumably mediated by direct ionisation of DNA or water to cause ROS in the case of irradiation, and Fenton-reaction derived ROS in the case of ascorbate. In fact, the degree of DSBs in response to combination treatment is similar to individual treatments, only slightly more at 2.5 -fold more than control.

Cells treated with ascorbate in PBS rather than media did not have a significant increase in the amount of DSBs compared with control, only 1.2-fold greater on average. This validated the need for trace transition metals in solution while treating with ascorbate, and supported the hydrogen peroxide mechanism.

In Figure 31, the same comet tail combinations experiment is shown with the tail moment parameter. It follows roughly the same trend as that displayed in Figure 30, with ascorbate in PBS not increasing the degree of DSBs (only 1.1-fold greater than control), irradiation causing a moderate 3.2-fold increase in DSBs, and ascorbate alone and in combination with 
irradiation increasing the amount of DSBs more than irradiation, 5 and 3.8 fold increases respectively.

Consistent with other data, Figure 32 demonstrates that the percent tail DNA does not allow differentiation of treatment groups, with all groups being within 0.08 -fold of each other.

In conclusion, the neutral comet tail assay, a more direct measure of DSBs than $\mathrm{YH} 2 \mathrm{AX}$, has a similar but not equal trend to that histone marker when considering the effect of combination ascorbate and irradiation treatment, at least in GL261 cells. ؟H2AX (see ' $\mathrm{YH} 2 \mathrm{AX}$ ') reports ascorbate increasing DNA damage, although to a slightly lesser degree than irradiation alone, and combination treatment increasing that measure more than either treatment individually. In contrast, the comet tail assay seems to support a more influential role for ascorbate. While combination treatment still causes the most DSBs in two of the three metrics used here (tail length and percentage tail DNA), ascorbate is shown as increasing DSBs to a greater degree than irradiation alone, in the context of the GL261 cells. 


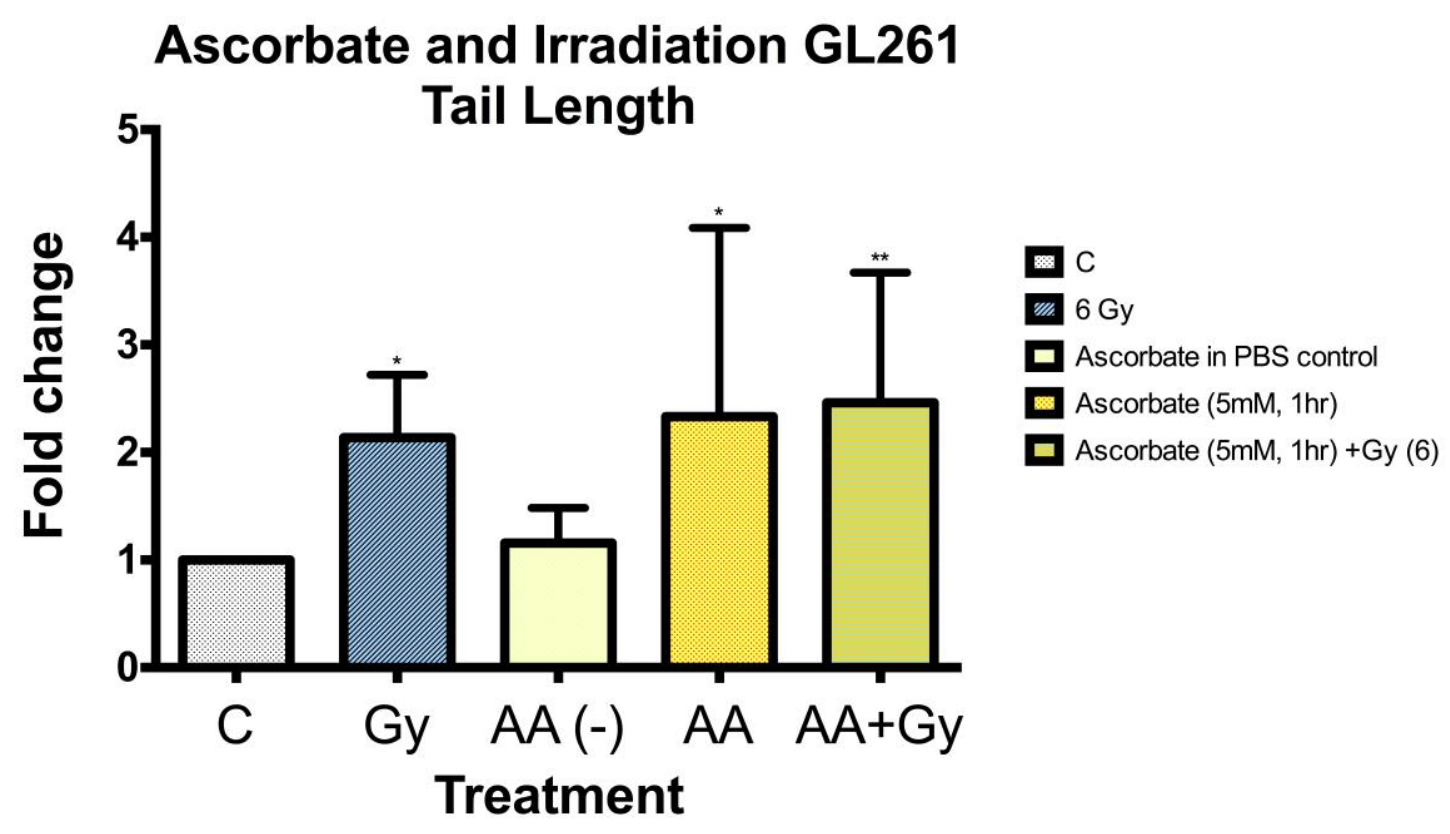

Figure 30. Single cell gel electrophoresis combination treatment tail lengths

Single cell gel electrophoresis combination treatment tail lengths (fold change + Standard Error of the Mean [SEM]) with GL261 cells. Cells were exposed to $5 \mathrm{mM}$ of ascorbate for an hour in either PBS as a negative control (AA(-)) or DMEM media (AA). Combination cells were treated with 6 Gy of irradiation at 1-hour post-ascorbate. All cell treatments were then combined with agarose and applied to pre-agarose coated slides. Cell-containing agarose was allowed to dry for 30 minutes on slides before lysis. Graph contains data from 9 independent experiments, and each treatment group has a sample size of between 3 and 9 experiments. All treatments except ascorbate in PBS were statistically significantly higher than control (P values: 6 Gy 0.0191, AA in PBS 0.1337, AA 0.0361, AA+Gy 0.0023. Graph and statistics produced with Prism V6.0. 


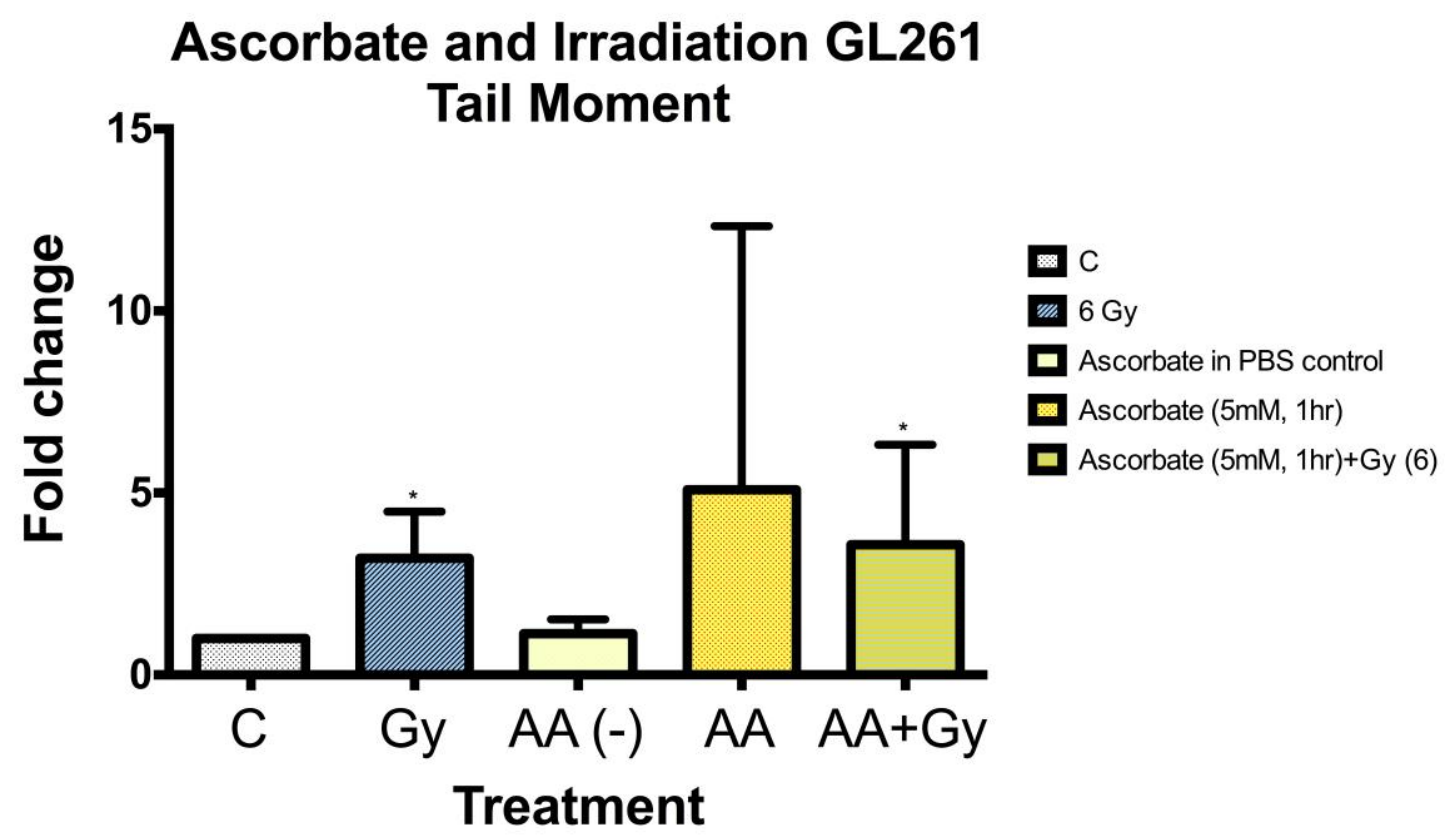

Figure 31. Single cell gel electrophoresis combination treatment tail moments

Single cell gel electrophoresis combination treatment tail lengths (fold change + Standard Error of the Mean [SEM]) with GL261 cells. Cells were treated as described in Figure 30. Graph contains data from 9 independent experiments, and each treatment group has a sample size of between 3 and 9 experiments. Treatments except ascorbate, and ascorbate in PBS were statistically significantly higher than control (P values: 6 Gy 0.0343, AA in PBS 0.2717, AA 0.1055, AA+Gy 0.0104. Graph and statistics produced with Prism V6.0. 


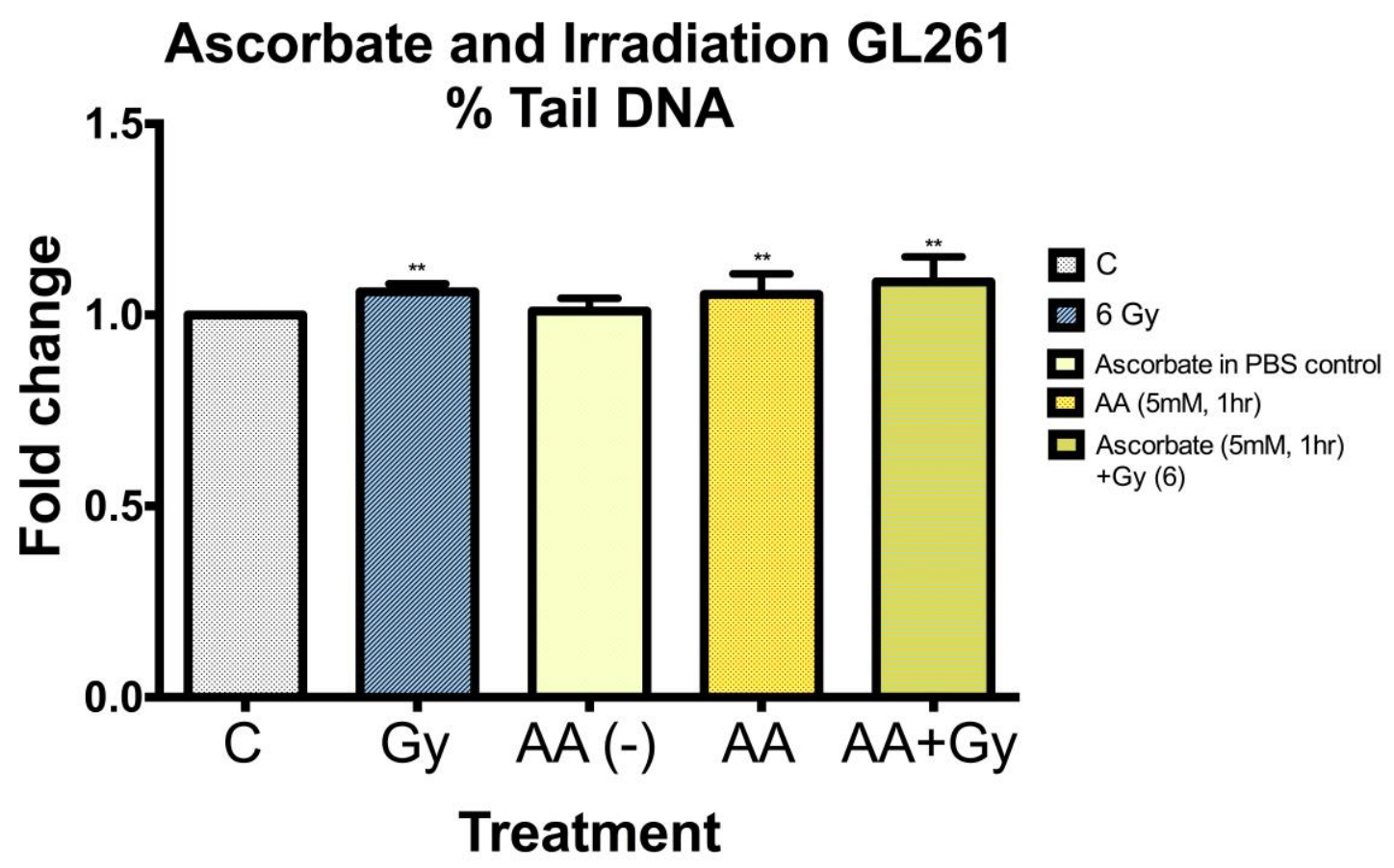

Figure 32. Single cell gel electrophoresis combination treatment percent tail DNA

Single cell gel electrophoresis combination treatment percent tail DNA (fold change + Standard Error of the Mean [SEM]) with GL261 cells. Cells were treated as described in Figure 30. Graph contains data from 9 independent experiments, and each treatment group has a sample size of between 3 and 9 experiments. Treatments except ascorbate in PBS were statistically significantly higher than control (P values: 6 Gy 0.0015, AA in PBS 0.3253, AA 0.0089, AA+Gy 0.0013. Graph and statistics produced with Prism V6.0. 


\section{Analysis of DNA Damage In Vivo}

To achieve the ultimate aim of this project, namely to determine the radioprotective or radiosensitive role of ascorbate in vivo, the second objective of the project was to undertake similar DNA damage analysis to that done on in vitro cells on in vivo tissue. To this end, an intracranial murine model of GBM utilising GL261 cells was established in C57BL/6 immunocompetent mice, mice were treated with the various treatments, and brains were harvested and markers of DNA damage assessed with immunofluorescence assays. Studying the same markers of DNA damage in vivo as investigated in vitro, in the same cell line, allows direct comparison of the particular DNA damage patterns. Therefore, it can then be determined whether ascorbate generates a pro-oxidant effect, enhancing radiotherapy, whether there is no effect on radiation-induced DNA damage, or whether the anti-oxidant activity inhibits radiotherapy.

\section{In Vivo Experimental Outline}

Two independent sets of experiments were run. Mice were implanted with $2 \mu \mathrm{L}$ of a $1.25 \times 10^{7} / \mathrm{mL}$ solution of cells, thus $2.5 \times 10^{4}$ cells total. Tumour tissue was collected after first a short-term 2-3hr exposure to ascorbate and radiation, and a longer-term $24 \mathrm{hr}$ exposure. Figure 33 is a diagrammatic representation of the experimental design timeline for these two experiments. In both experiments, mice underwent identical intracranial surgery once older than 8 weeks. Figure 34 compares the weights of mice on the day of intracranial surgery with weights on the day of treatment. Although there was some variation in mouse weights between these times, there was no significant difference in weights on the day of surgery and the day of treatment (paired t-test p value: 0.5329 ). Therefore, the tumour had not affected mice weights by the 18 or 19-day time point.

In the short term treatment experiment, on day 18 post-surgery mice were weighed, assigned to a treatment group, and treated with either nothing, $2 \mathrm{mg} / \mathrm{kg}$ ascorbate, $13 \mathrm{~Gy}$ of irradiation (13 Gy irradiation setting results in a dose of $\sim 10$ Gy to the head (Grasso, Fabre et al. 2014)), or both treatments. Ascorbate was given either before or immediately after 
irradiation. Mice were assigned to treatment groups such that all groups contained representation from each individual mouse box, to control for potential inter-box variability. All mice were culled between 1 and 2 hours after irradiation treatment.

For the longer term treatment experiment, mice were weighed, assigned to a treatment group, and treated on day 19 post-surgery with either nothing, $2 \mathrm{mg} / \mathrm{kg}$ ascorbate, $13 \mathrm{~Gy}$ of irradiation (10 Gy to the head), or both treatments with ascorbate first, followed by irradiation. The longer term treatment experiment was staggered such that only one box and one mouse of each treatment group were treated on any given day, in order to eliminate any inter-box or inter-day bias. Mice were culled 24 hours post irradiation treatment. 
Time in days:

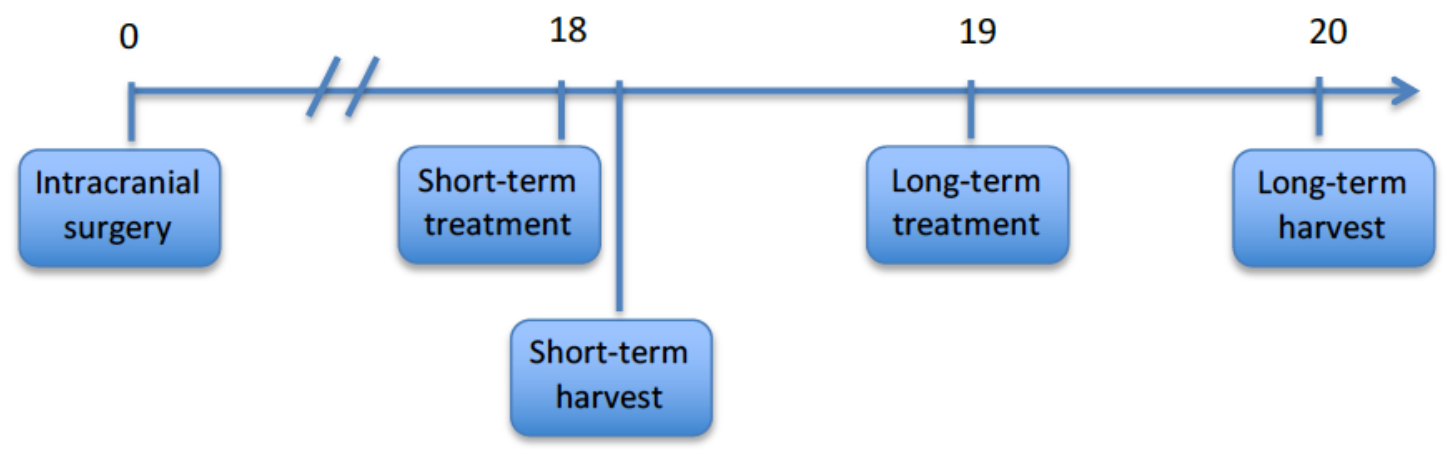

Figure 33. In Vivo Experimental Plan 


\section{Experimental Mouse Weights}

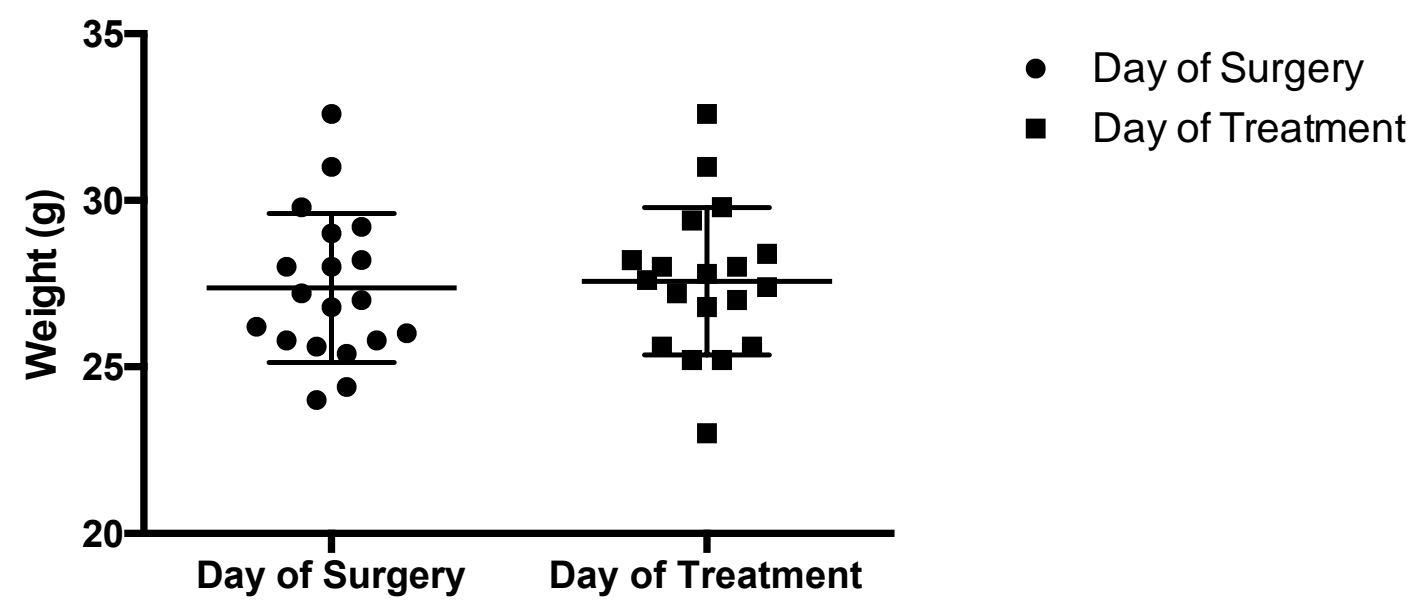

Figure 34. Experimental mouse weights

Experimental mouse weights (male) on the day of surgery and the day of treatment. $n=19$. There was no significant difference between weights on the two days. Paired t test: $\mathrm{P}$ value= 0.5329 


\section{Immunofluorescence Assay}

Once culled, mice were perfused with formalin and PBS, and brains were harvested and incubated overnight in formalin before being transferred to $70 \%$ ethanol. Brain tissue was processed, embedded in paraffin wax, and $5 \mu \mathrm{m}$ sections were cut.

\section{Initial Immunofluorescence Results}

In order to determine whether the same pattern of DNA damage occurred in vivo as in vitro, immunofluorescence (IF) assays were carried out to attempt to detect and quantify $\mathrm{\Upsilon H}_{2} \mathrm{AX}$ signal from in vivo brain samples. Initial experiments used normal, non-tumour brain tissue areas and compared only brains that had received no treatment to brains that had received irradiation only.

Figure 35 is a representative image of unirradiated, non-tumour brain tissue that was stained with $\Upsilon \mathrm{H} 2 \mathrm{AX}$. Brain tissue was processed as described in 'Methods', but blocked with 1\% BSA, and stained with YH2AX antibody at a dilution factor of 1:200. As predicted, very little YH2AX positive punctae are visible, which indicates the relatively low levels of DNA damage present in the untreated mouse brain. DAPI stain, a commonly used marker of DNA, applied to the same tissue allows delineation of the boundaries of brain nuclei, and therefore gives an indication as to whether the $\mathrm{rH} 2 \mathrm{AX}$ is within the nucleus. Of the little numbers of punctae visible, a small proportion overlap with DAPI and can so be interpreted as true $\mathrm{YH} 2 \mathrm{AX}$ staining present within the nucleus.

In Figure 36, a representative image of normal non-tumour, irradiated tissue, from the same experiment as Figure 35 and stained with YH2AX is displayed. The mouse was involved in a short term treatment experiment and thus brain was harvested within 1-2 hours of irradiation (see 'In Vivo Experimental Outline'). Relatively more $\mathrm{rH} 2 \mathrm{AX}$ positive regions can be identified in the irradiated brain sample as compared with the untreated sample. The large amount of punctae indicates the high 
amount of DNA damage. As with the unirradiated brain sample, a proportion of punctae are visible within the nuclei.

Nine independent immunofluorescence assays comparing control to irradiated brains were carried out using tissue from the two in vivo experiments described previously and staining for $\mathrm{\Upsilon H} 2 \mathrm{AX}$. All showed a comparable difference in DNA-damage-indicating positive stain between the two treatments, with irradiated brain displaying consistently more positive punctae (data not shown). This difference was identifiable by eye.

In conclusion, a comparison of irradiated and non-irradiated brain stained with a fluorescent antibody marker of $\Upsilon \mathrm{H} 2 \mathrm{AX}$ indicates that $10 \mathrm{~Gy}$ of irradiation to the murine head causes an observable increase in $\mathrm{rH} 2 \mathrm{AX}$ positive areas, strongly suggesting an increase in DNA damage caused to brain cells in this situation. 


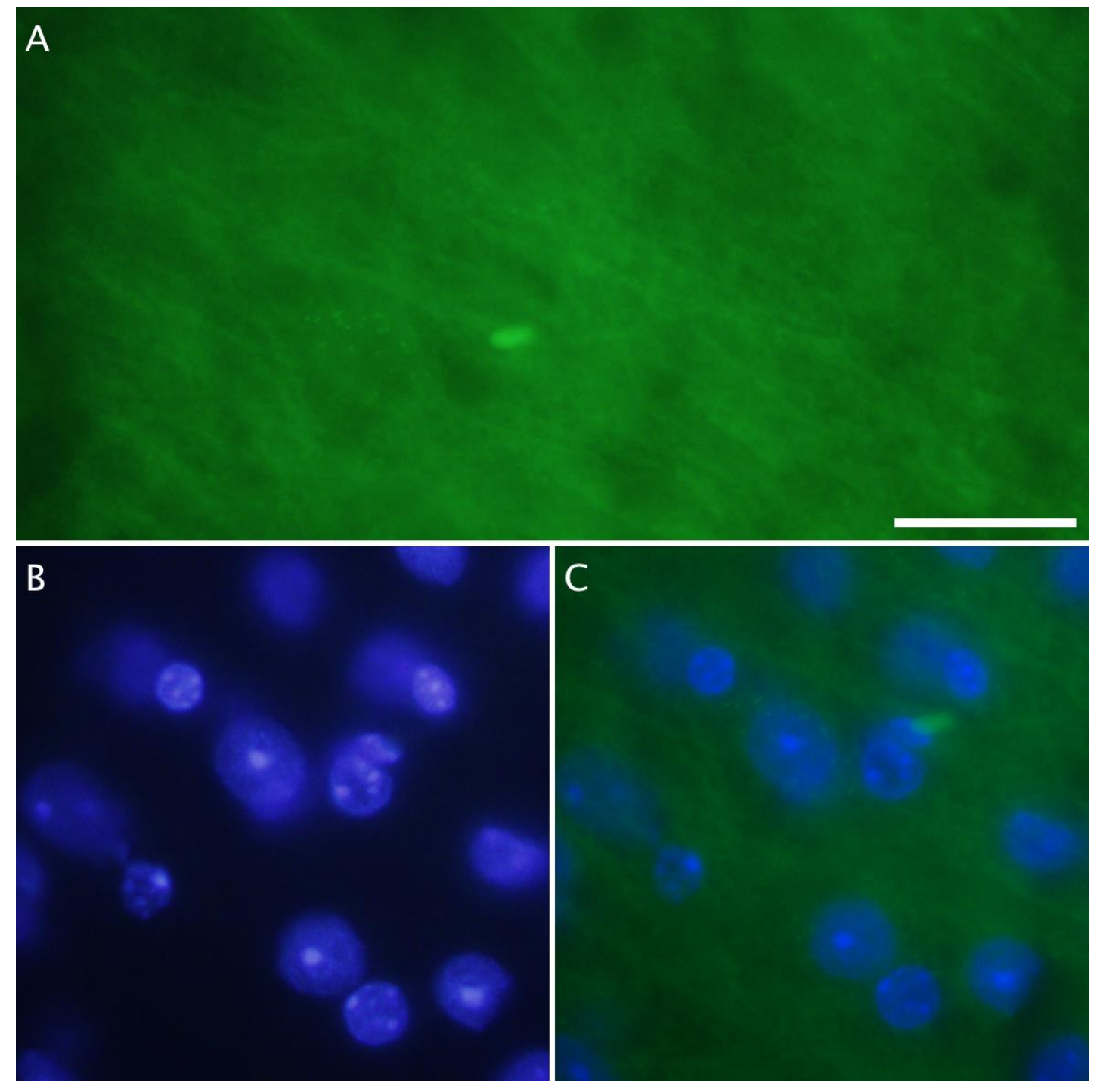

Figure 35. Normal, untreated brain tissue has little $\mathrm{YH} 2 \mathrm{AX}$ positive staining

Representative images of normal untreated brain tissue stained with $\Upsilon \mathrm{H} 2 \mathrm{AX}$ and DAPI.

A. Normal non-tumour untreated brain tissue stained with $\mathrm{YH} 2 \mathrm{AX}$ and imaged at 100x objective. A large amount of background autofluorescence is visible as generalised green colour. A small amount of $\Upsilon \mathrm{H} 2 \mathrm{AX}$ positive staining can be seen from basal DNA damage. B. The same field of view stained with DAPI nuclear stain. C. The same field of view displaying an overlay of $\Upsilon \mathrm{H} 2 \mathrm{AX}$ and DAPI to discern punctae that are within nuclei. The scale bar of 200 pixels applies to all three images and corresponds to $12.9 \mu \mathrm{m}$. Images A and B were unaltered. The brightness on Image $\mathrm{C}$ was increased to allow visualisation of $\Upsilon \mathrm{H} 2 \mathrm{AX}$ positive punctae within the bounds of DAPI nuclear stain. Produced using the Image J FigureJ plugin. 


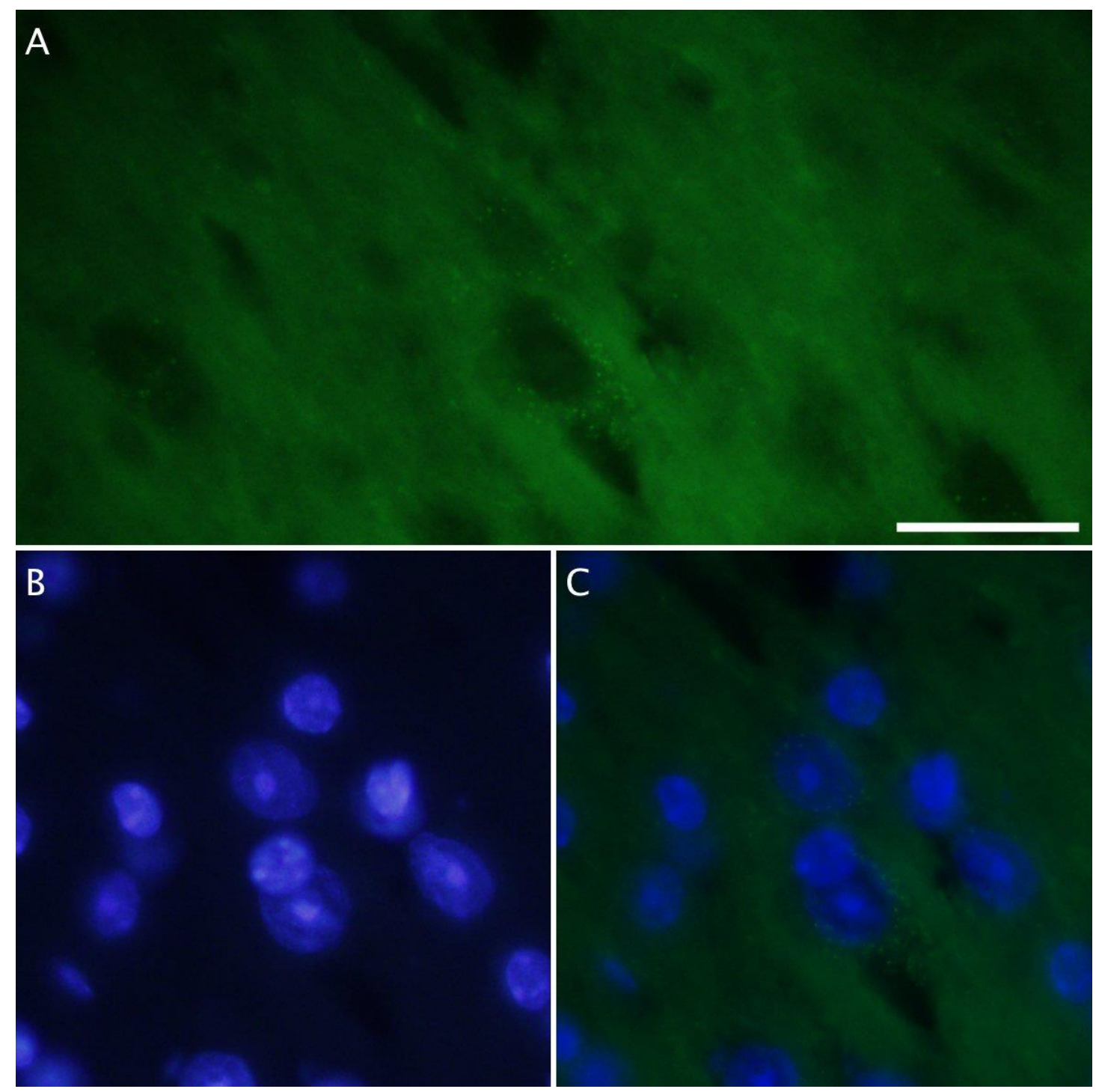

Figure 36. Normal, irradiated brain tissue has an increase in basal $\mathrm{YH} 2 \mathrm{AX}$ positive staining

Representative images of normal irradiation treated brain tissue stained with $\Upsilon \mathrm{H} 2 \mathrm{AX}$ and DAPI.

A. Normal non-tumour brain tissue irradiated with $10 \mathrm{~Gy}$ as described in methods, stained with YH2AX and imaged at 100x objective. As with Figure 35, a large amount of background autofluorescence is visible as generalised green colour. $\mathrm{YH} 2 \mathrm{AX}$ positive punctae can be seen, to a relatively higher degree than Figure 35. B. The same field of view stained with DAPI nuclear stain. C. The same field of view displaying an overlay of $\mathrm{YH} 2 \mathrm{AX}$ and DAPI to discern punctae that are within nuclei. ҮH2AX positive punctae are visible in and around DAPI positive nuclei. The scale bar of 200 pixels applies to all three images and corresponds to $12.9 \mu \mathrm{m}$. Images A and B were unaltered. The brightness on Image C was increased to allow visualisation of $\mathrm{CH}_{2} \mathrm{AX}$ positive punctae within the bounds of DAPI nuclear stain. Produced using the ImageJ FigureJ plugin. 


\section{Troubleshooting}

Due to the abundance of background fluorescence seen in the initial IF experiments, many methods were tried in order to improve the clarity of IF pictures and thus data, to allow potential quantification of the results described. This troubleshooting was carried out comparing control to irradiated normal brain sections.

Table 6 details the different alterations to the $\mathrm{YH} 2 \mathrm{AX}$ IF protocol that were attempted. No alteration decreased autofluorescence to allow determination of $\mathrm{YH} 2 \mathrm{AX}$ positive punctae from generalised autofluorescence. The only alterations that did decrease autofluorescence were an alternative AlexaFluor 647 conjugated $\mathrm{YH} 2 \mathrm{AX}$ antibody, which revealed no fluorescence unless at high exposure, in which case fluorescence was generalised and red, or treatment after antibody incubation with a $0.1 \%$ solution of Sudan Black, which reduced all fluorescence save fluorescence of blood and blood vessels. 
Table 6. Optimisation of Immunofluorescence Assay

\begin{tabular}{|c|c|c|c|}
\hline Alteration & Explanation & & Outcome \\
\hline \multirow[t]{7}{*}{ Antibody } & \multirow{7}{*}{$\begin{array}{l}\text { Titrations. } \\
\text { ҮH2AX antibody was used at } \\
\text { the following dilution factors } \\
\text { (concentration): }\end{array}$} & $1: 2000 \quad(12.5 \mathrm{ng} / \mathrm{mL})$ & \multirow{7}{*}{$\begin{array}{l}\text { No antibody concentration decreased } \\
\text { autofluorescence. Titrations higher than 1:50 } \\
\text { appeared to cause positive signal to be brighter. }\end{array}$} \\
\hline & & $1: 1000 \quad(25 \mathrm{ng} / \mathrm{mL})$ & \\
\hline & & $1: 500 \quad(50 \mathrm{ng} / \mathrm{mL})$ & \\
\hline & & $1: 200 \quad(125 \mathrm{ng} / \mathrm{mL})$ & \\
\hline & & $(250 \mathrm{ng} / \mathrm{mL})$ & \\
\hline & & $1: 50 \quad(500 \mathrm{ng} / \mathrm{mL})$ & \\
\hline & & $1: 20 \quad(1.25 \mu \mathrm{g} / \mathrm{mL})$ & \\
\hline & $\begin{array}{l}\text { Choice of Fluorophore. } \\
488 \text { and } 647 \text { conjugated } \\
\text { \H2AX antibody were used }\end{array}$ & $\begin{array}{l}\text { 488: Green fluorescence is } \\
\text { overcome by tissue } \\
\text { autofluorescence. } \\
\text { 647: Fluoresces in the red } \\
\text { channel. }\end{array}$ & $\begin{array}{l}\text { All concentrations of } 488 \text { antibody caused } \\
\text { autofluorescence (see above). } \\
\text { Dilutions of } 647 \text { antibody (1:1000, 1:500, 1:250, } \\
1: 50,1: 20 \text { ), did not show fluorescence in the Cy5 } \\
\text { filter set without extremely high exposure. } \\
\text { Exposure of high magnitude caused a } \\
\text { generalised red fluorescence. The } 647 \text { was } \\
\text { validated and was functional via flow cytometry } \\
\text { (data not shown). }\end{array}$ \\
\hline & Incubation & $\begin{array}{l}\text { Overnight at } 4^{\circ} \mathrm{C} \text { and } \\
1 \text { hour at room temperature. }\end{array}$ & $\begin{array}{l}\text { Both methods of antibody incubation were } \\
\text { similar. Neither method decreased } \\
\text { autofluorescence. }\end{array}$ \\
\hline \multirow[t]{4}{*}{ Antigen retrieval } & \multirow{3}{*}{$\begin{array}{l}\text { Three types of antigen } \\
\text { retrieval }\end{array}$} & $10 \mathrm{mM}$ sodium citrate, $\mathrm{pH} 6$ & \multirow{4}{*}{$\begin{array}{l}\text { No antigen retrieval method decreased } \\
\text { autofluorescence. }\end{array}$} \\
\hline & & EDTA buffer, pH 8 & \\
\hline & & Tris EDTA buffer, $\mathrm{pH} 9$ & \\
\hline & Time and Temperature & $\begin{array}{l}\text { Varied the time and } \\
\text { temperature of antigen retrieval } \\
\text { from } 95^{\circ} \mathrm{C} \text { for } 10 \text { minutes to } \\
85^{\circ} \mathrm{C} \text { for } 60 \text { mins. }\end{array}$ & \\
\hline
\end{tabular}




\begin{tabular}{|c|c|c|c|}
\hline Agitation & During processing of slides & $\begin{array}{l}\text { Investigated agitating coplin } \\
\text { jars during ethanol and wash } \\
\text { steps. }\end{array}$ & Did not decrease autofluorescence. \\
\hline \multirow[t]{3}{*}{ Block } & Concentration & $\begin{array}{l}\text { Changed the concentration of } \\
\text { block from } 1 \% \text { to } 5 \% \text { BSA. }\end{array}$ & \multirow[t]{3}{*}{$\begin{array}{l}\text { All blocking methods were similar. Neither } \\
\text { method decreased autofluorescence. }\end{array}$} \\
\hline & Incubation & $\begin{array}{l}\text { Investigated overnight at } 4^{\circ} \mathrm{C} \\
\text { and } 1 \text { or } 2 \text { hours at room } \\
\text { temperature. }\end{array}$ & \\
\hline & Donkey serum & $\begin{array}{l}\text { Attempted } 2 \% \text { donkey serum } \\
\text { with } 1 \% \text { BSA block. }\end{array}$ & \\
\hline Sodium borohydride & $\begin{array}{l}\text { An additional antigen } \\
\text { retrieval chemical }\end{array}$ & $\begin{array}{l}\text { Added a } 0.5 \% \text { sodium } \\
\text { borohydride retrieval step }\end{array}$ & Did not decrease autofluorescence. \\
\hline Sudan Black & $\begin{array}{l}\text { Commonly used to quench } \\
\text { tissue autofluorescence }\end{array}$ & $\begin{array}{l}\text { Added a } 1 \% \text { Sudan Black in } \\
\text { TBST after antibody } \\
\text { incubation }\end{array}$ & $\begin{array}{l}\text { Decreased autofluorescence, yet also eliminated } \\
\text { all signal other than red blood cells. }\end{array}$ \\
\hline Wash buffer & $\begin{array}{l}\text { Phosphate Buffered Saline } \\
\text { (PBS) or Tris Buffered Saline } \\
\text { and Tween-20 (TBST) }\end{array}$ & $\begin{array}{l}\text { Switched to from PBS to } \\
\text { TBST for washes, dilution of } \\
\text { BSA, dilution of antibodies }\end{array}$ & Did not decrease autofluorescence. \\
\hline Confocal & $\begin{array}{l}\text { Looked at some slides with a } \\
\text { confocal microscope }\end{array}$ & $\begin{array}{l}\text { Try and determine whether the } \\
\text { rH2AX staining was within } \\
\text { the nucleus }\end{array}$ & $\begin{array}{l}\text { Allowed a more detailed image of the } \\
\text { fluorescence but could not confirm that } \mathrm{YH} 2 \mathrm{AX} \\
\text { positive signalling was contained within the } \\
\text { nuclei, as indicated by DAPI staining }\end{array}$ \\
\hline
\end{tabular}




\section{Tumour Immunofluorescence}

Due to time constraints, it was not possible to identify a solution that would eliminate autofluorescence without blocking true fluorescence, before progressing to an attempted analysis of tumour tissue.

Tumours were located by sectioning $2 \mathrm{~mm}$ in from the midline of each hemisphere. Some regions of tumour were more easily identifiable by areas of necrosis. Slides containing tumour tissue were processed through an immunofluorescence protocol described in 'Methods' and imaged as soon as possible, always within 48 hours.

DNA-bound DAPI stain was used to identify regions of tumour, indicated by the densely packed and heterogeneous nature of tumour nuclei. Figure 37 characterises nuclei with representative pictures of DAPI stained tumour nuclei taken at 3 different objectives. These images confirm presence of established GBM tumour in mouse brains in response to GL261 inoculation.

YH2AX and 8-OHdG was used to stain tumour tissue of each treatment in order to establish the specific types of DNA damage caused by ascorbate, irradiation or combinations of both in either sequence.

Figures 38 to 42 show a sequence of representative images of each treatment group covered in these experiments, stained with $\mathrm{rH} 2 \mathrm{AX}$ and DAPI. Considerable background autofluorescence impeded identification of subtle and quantifiable trends, although overall differences can be discerned. It was discovered that both ascorbate and irradiation alone caused an increase in $\mathrm{YH} 2 \mathrm{AX}$ positive punctae in brain tumour tissue, (Fig 39 and 40). Interestingly, in the groups of mice that received both ascorbate and irradiation treatment, both the group that were given ascorbate as pretreatment and the group that received ascorbate immediately after irradiation treatment, apparently less $\mathrm{\Upsilon H} 2 \mathrm{AX}$ positive areas are observable (Figure 41 and 42). The two groups that received both treatments were 
comparable in the low degree of $\Upsilon \mathrm{H} 2 \mathrm{AX}$ staining seen, and potentially on par with the basal level of $\mathrm{YH} 2 \mathrm{AX}$ observed in control brain cells (Figure $38)$.

Figures 43 to 47 are representative images of the tumour brain tissue of each treatment group, stained with 8-OHdG and DAPI. Again, background autofluorescence hampers observation, yet some differences can be seen. For instance, ascorbate only treatment (Figure 44) in general displays distinctive, widespread 8-OHdG staining throughout the tumour tissue that is of a noticeably different quality to other treatment groups, even ascorbate combination treated tissue (Figure 46 and 47). As with the ҮH2AX marker, irradiation alone caused an increase in $8-\mathrm{OHdG}$ positive punctae in brain tumour tissue, (Fig 43). In the groups of mice that received both ascorbate and irradiation treatments, again, less $8-\mathrm{OHdG}$ positive areas are observable (Figure 46 and 47).

In summary, preliminary in vivo evidence, although not fully optimised, indicates that in both $\mathrm{YH}^{\mathrm{AXX}}$ and 8-OHdG markers of damage to DNA, neither ascorbate pre or post irradiation treatment functions as a prooxidant and increases DNA damage caused by irradiation. On the contrary, combination treatments may in fact decrease the DNA damaging effect of irradiation as measured with these two markers, and thus potential therapeutic potential. 


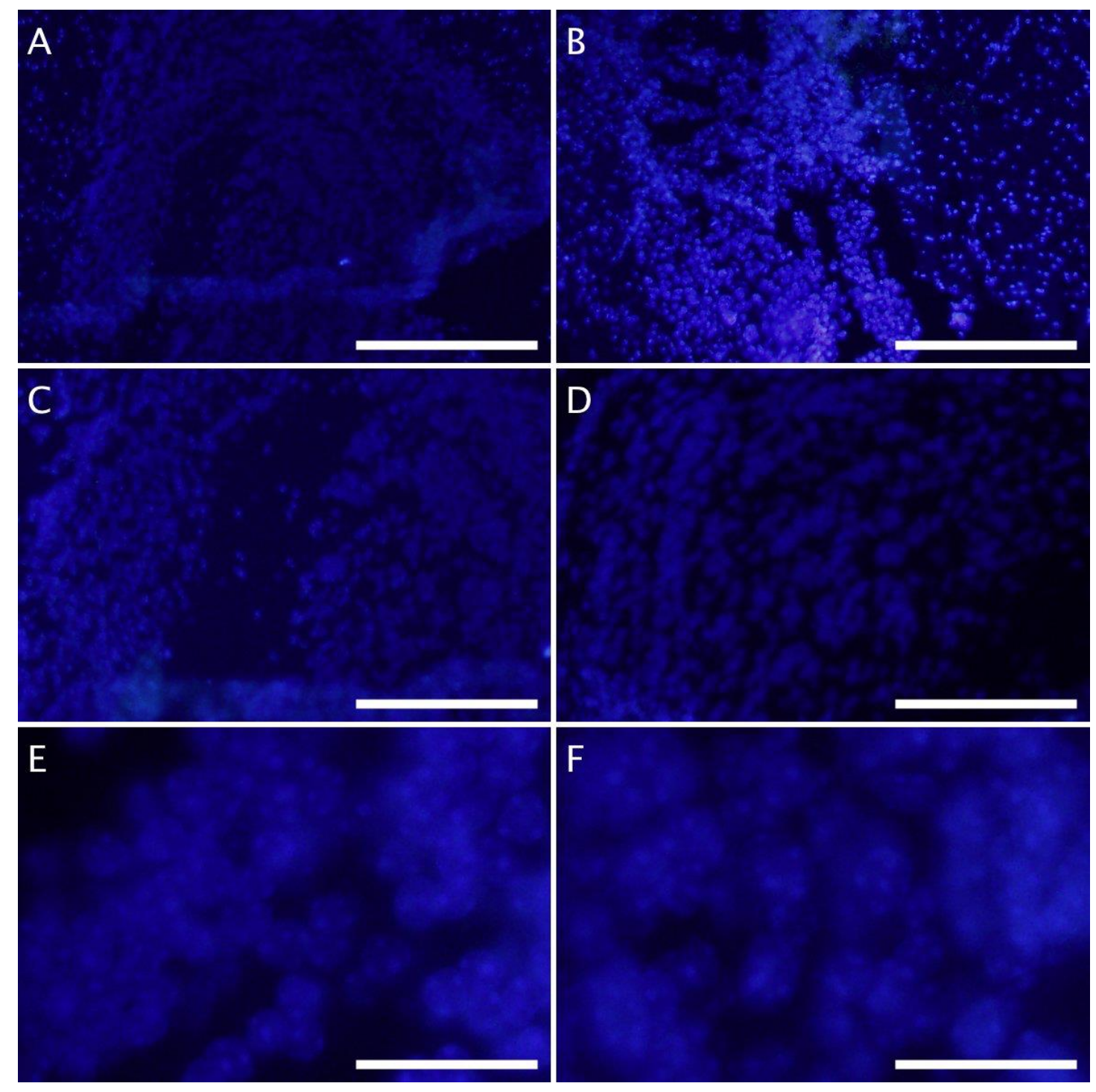

Figure 37. Representative Images of DAPI stained tumour tissue from untreated brain.

A and B. Untreated tumour brain tissue stained with DAPI and imaged at 10x objective. The scale bar of 200 pixels corresponds to $129 \mu \mathrm{m} \mathrm{C}$ and D. Untreated tumour brain tissue stained with DAPI and imaged at 20x objective. The scale bar of 200 pixels corresponds to $64.5 \mu \mathrm{m}$. E and F. Untreated tumour brain tissue stained with DAPI and imaged at 100x objective. The scale bar of 200 pixels corresponds to $12.9 \mu \mathrm{m}$. All images were unaltered for contrast and brightness. Produced using the ImageJ FigureJ plugin. 


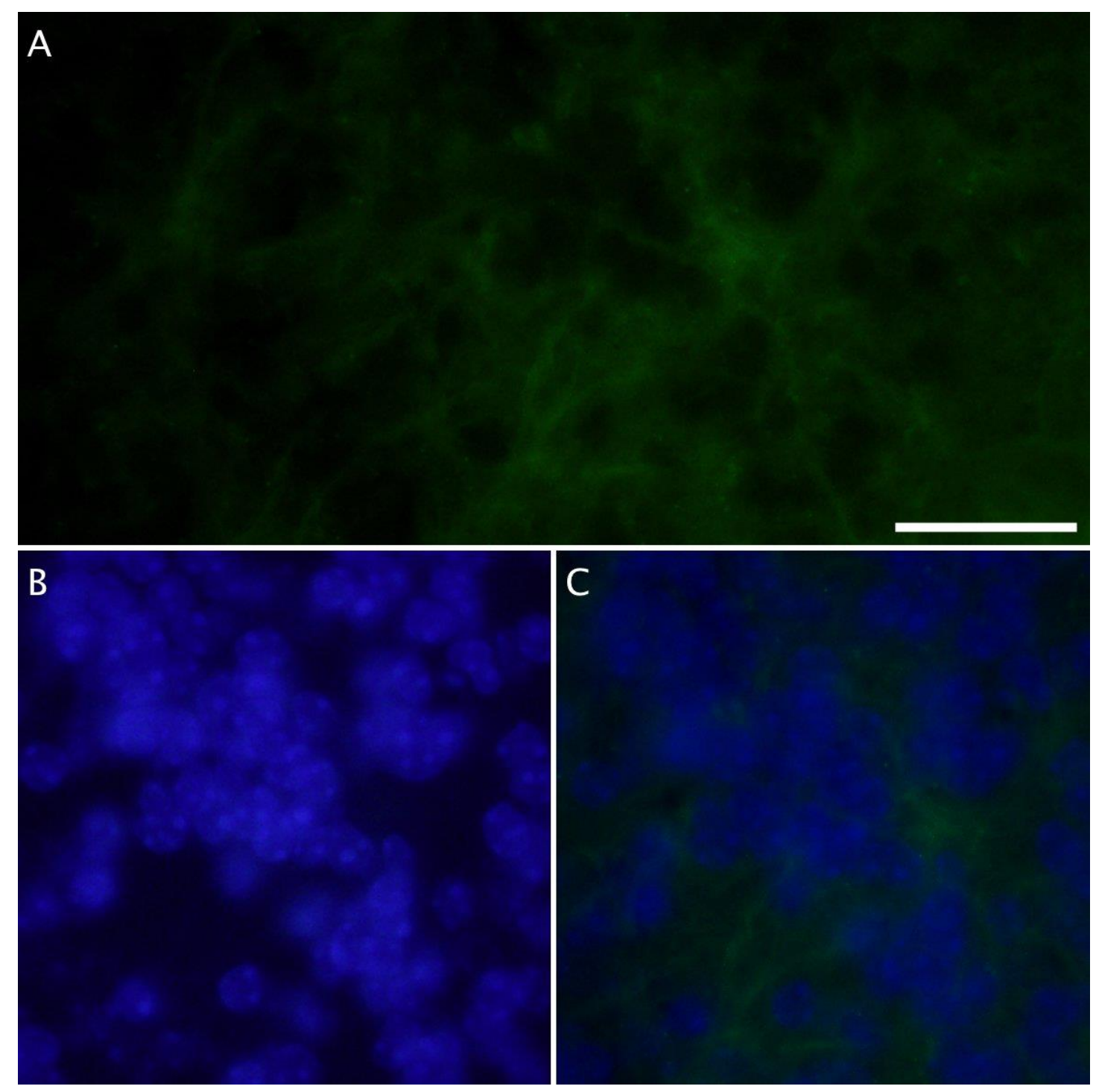

Figure 38. Representative Images of $\Upsilon \mathrm{H} 2 \mathrm{AX}$ and DAPI stained tumour tissue from untreated brain

A. Untreated control tumour brain tissue stained with $\mathrm{YH} 2 \mathrm{AX}$ and imaged at 100x objective. A large amount of background autofluorescence is visible as generalised green colour. Some $\mathrm{YH} 2 \mathrm{AX}$ positive punctae can be seen, to a relatively lower degree than Figure 39. B. The same field of view stained with DAPI nuclear stain. C. The same field of view displaying an overlay of $\Upsilon \mathrm{H} 2 \mathrm{AX}$ and DAPI to discern punctae that are within nuclei. The scale bar of 200 pixels corresponds to $12.9 \mu \mathrm{m}$. Images A and B were unaltered; the brightness of the composite Image C was increased. Produced using the Image J FigureJ plugin. 

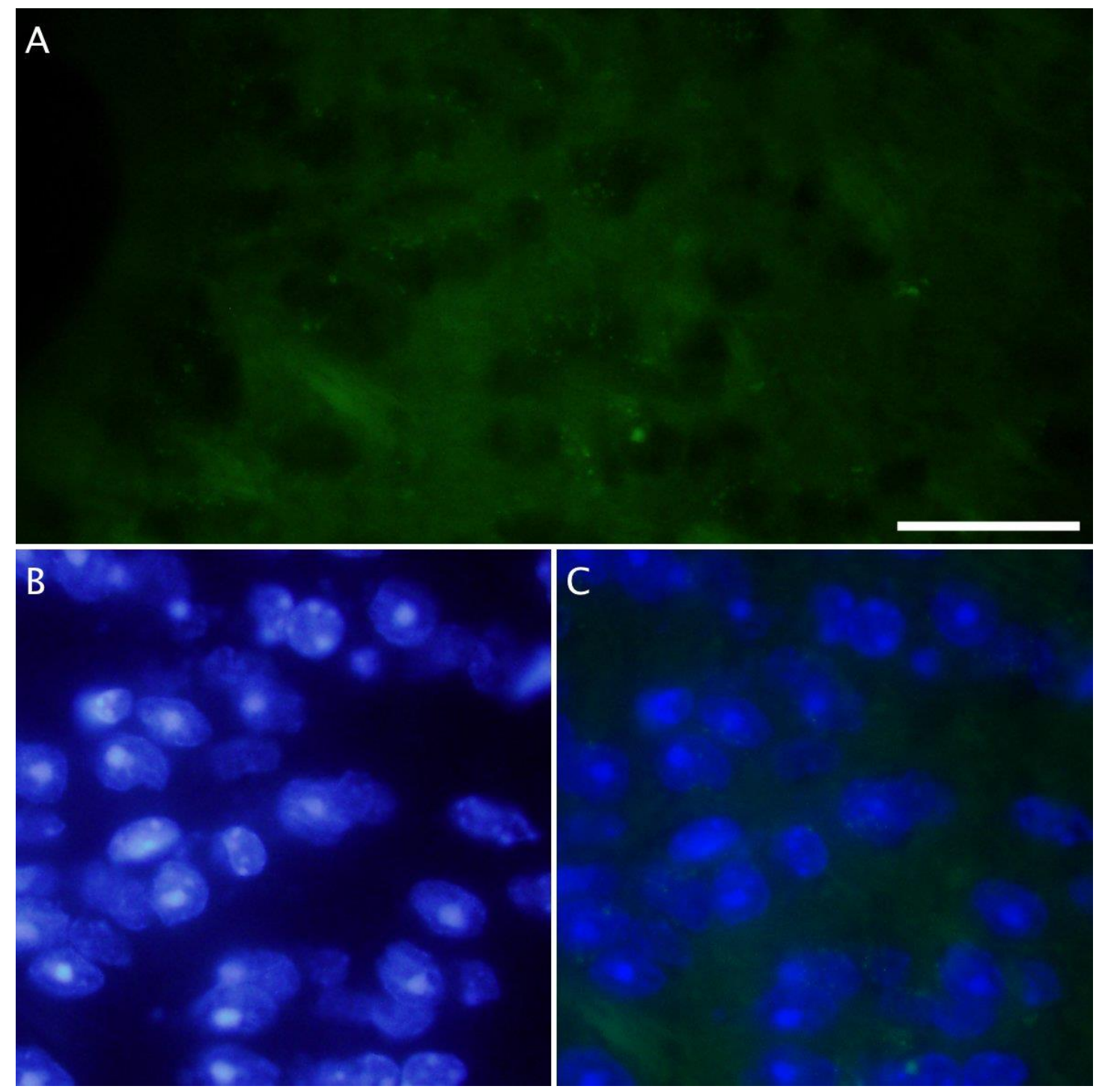

Figure 39. Representative Images of $\Upsilon \mathrm{H} 2 \mathrm{AX}$ and DAPI stained tumour tissue from ascorbate treated brain

A. Ascorbate treated tumour brain tissue stained with $\mathrm{YH} 2 \mathrm{AX}$ and imaged at 100x objective. A large amount of background autofluorescence is visible as generalised green colour. $\mathrm{YH} 2 \mathrm{AX}$ positive punctae are visible, to a greater degree than Figure 38. B. The same field of view stained with DAPI nuclear stain. C. The same field of view displaying an overlay of $\Upsilon \mathrm{H} 2 \mathrm{AX}$ and DAPI to discern punctae that are within nuclei. The scale bar of 200 pixels corresponds to $12.9 \mu \mathrm{m}$. Images A and B were unaltered; the brightness of the composite Image $\mathrm{C}$ was increased. Produced using the Image FigureJ plugin. 

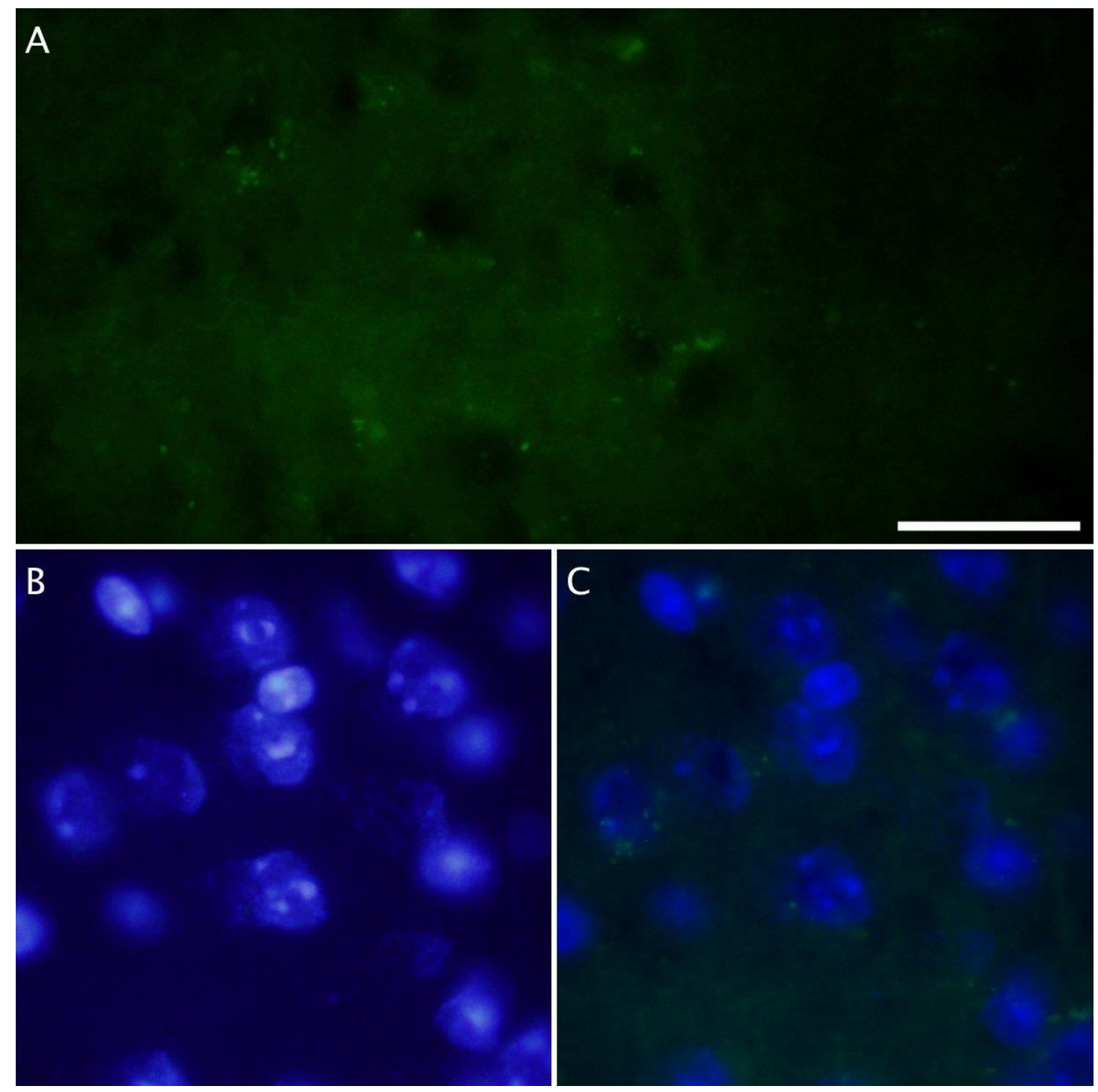

Figure 40. Representative Images of $\mathrm{YH} 2 \mathrm{AX}$ and DAPI stained tumour tissue from irradiated brain

A. Irradiation treated tumour brain tissue stained with $\mathrm{\Upsilon H} 2 \mathrm{AX}$ and imaged at 100x objective. A large amount of background autofluorescence is visible as generalised green colour. $\mathrm{YH} 2 \mathrm{AX}$ positive punctae are visible, to a greater degree than Figure 38. B. The same field of view stained with DAPI nuclear stain. C. The same field of view displaying an overlay of $\Upsilon \mathrm{H} 2 \mathrm{AX}$ and DAPI to discern punctae that are within nuclei. The scale bar of 200 pixels corresponds to $12.9 \mu \mathrm{m}$. Images A and B were unaltered; the brightness of the composite Image $\mathrm{C}$ was increased. Produced using the ImageJ FigureJ plugin. 

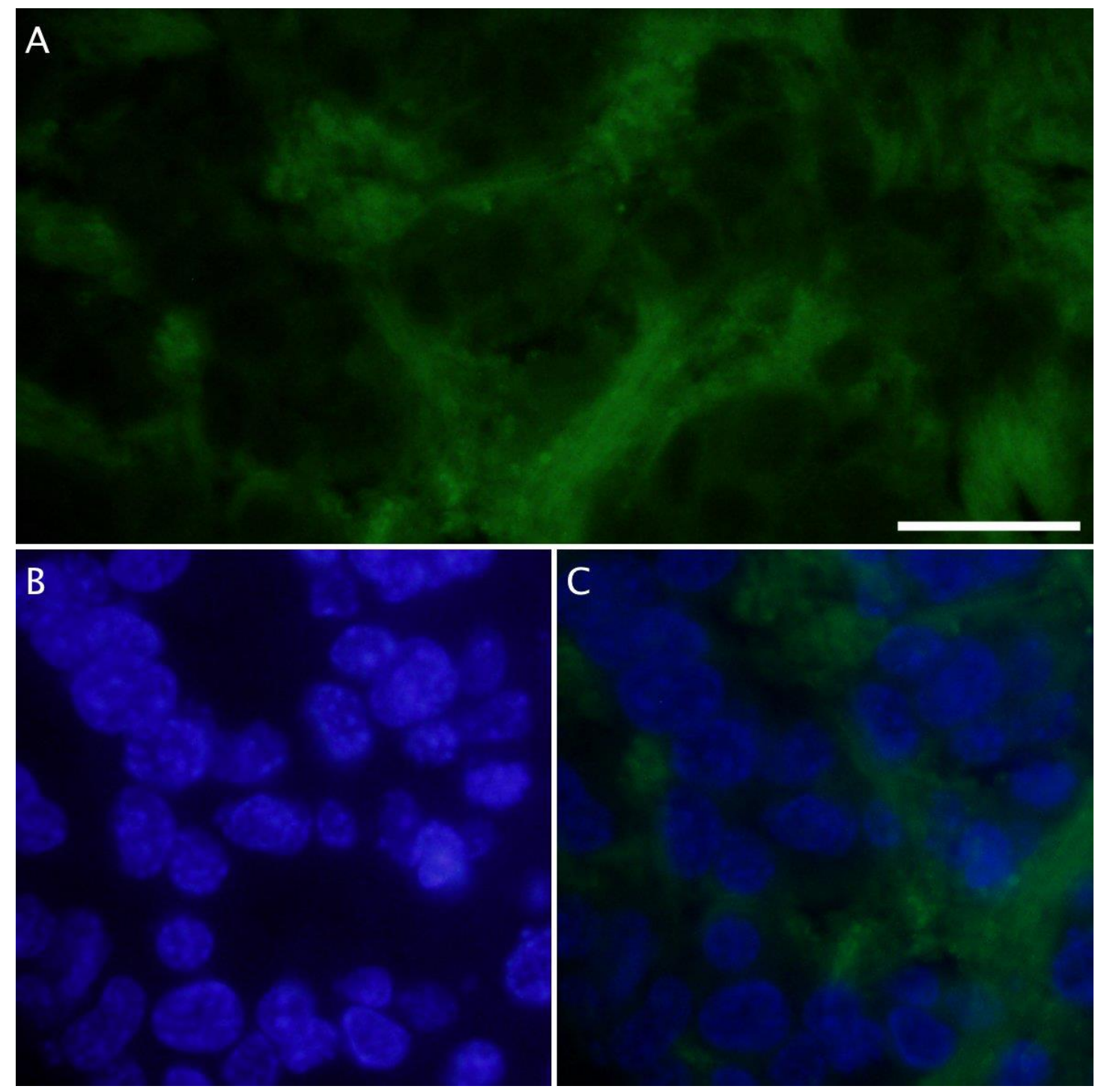

Figure 41. Representative Images of $\Upsilon \mathrm{H} 2 \mathrm{AX}$ and DAPI stained tumour tissue from ascorbate pre-treated, irradiated brain

A. Ascorbate pre-treatment and irradiation treated tumour brain tissue stained with $\mathrm{rH}_{2} \mathrm{AX}$ and imaged at 100x objective. A large amount of background autofluorescence is visible as generalised green colour. $\mathrm{YH} 2 \mathrm{AX}$ positive punctae are visible, but to a lesser degree than Figure 39 or Figure 40. B. The same field of view stained with DAPI nuclear stain. C. The same field of view displaying an overlay of $\Upsilon \mathrm{H} 2 \mathrm{AX}$ and DAPI to discern punctae that are within nuclei. The scale bar of 200 pixels corresponds to $12.9 \mu \mathrm{m}$. Images A and B were unaltered; the brightness of the composite Image C was increased. Produced using the ImageJ FigureJ plugin. 


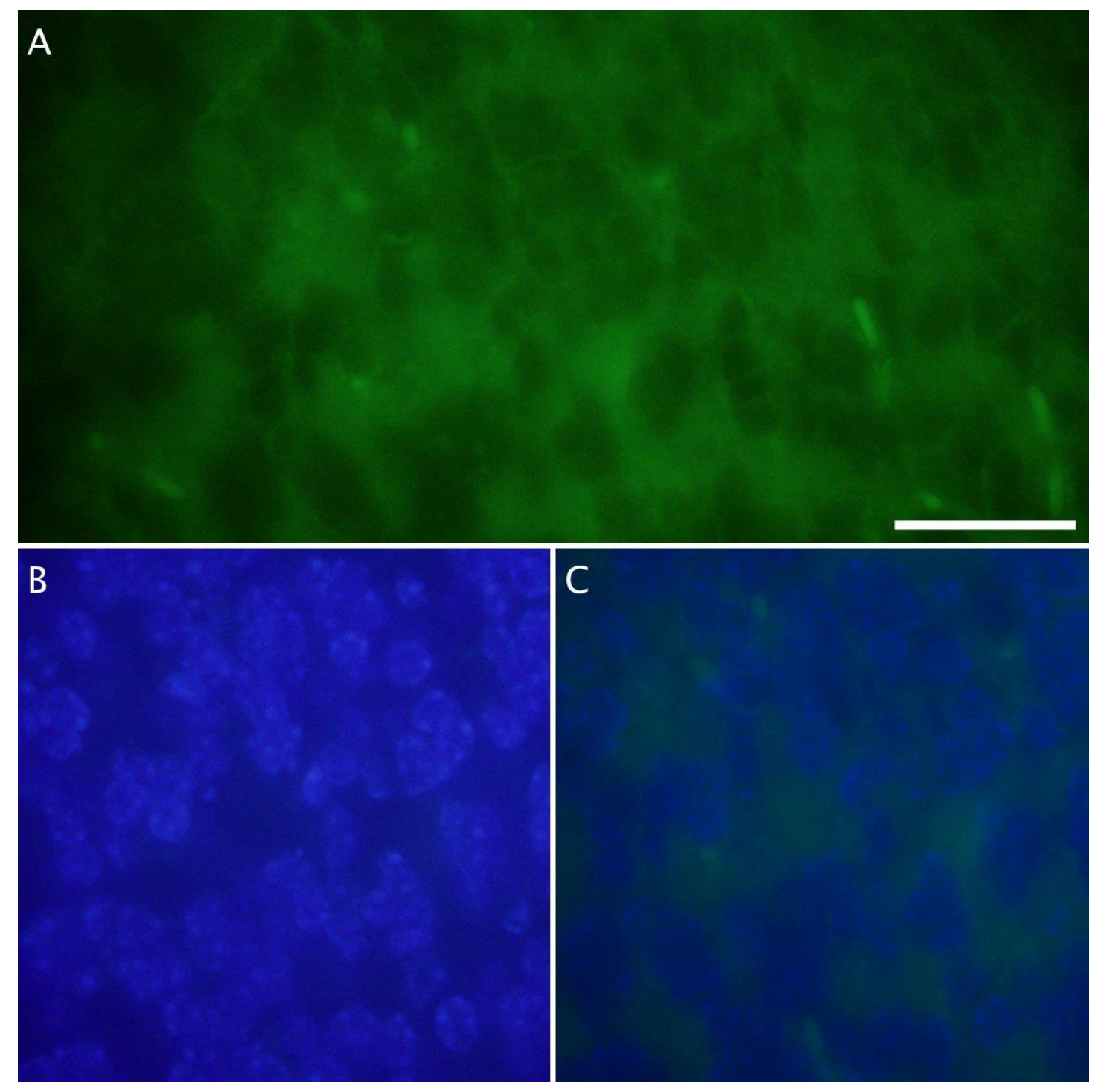

Figure 42. Representative Images of $\mathrm{YH} 2 \mathrm{AX}$ and DAPI stained tumour tissue from irradiated, ascorbate post-treated brain

A. Irradiation and ascorbate post-treatment treated tumour brain tissue stained with $\mathrm{YH} 2 \mathrm{AX}$ and imaged at 100x objective. A large amount of background autofluorescence is visible as generalised green colour. Very little, if any $\mathrm{YH} 2 \mathrm{AX}$ positive punctae are visible. B. The same field of view stained with DAPI nuclear stain. C. The same field of view displaying an overlay of $\mathrm{rH}_{2} \mathrm{AX}$ and DAPI to discern any punctae that are within nuclei. The scale bar of 200 pixels corresponds to 12.9 $\mu \mathrm{m}$. Images A and B were unaltered; the brightness of the composite Image $\mathrm{C}$ was increased. Produced using the ImageJ FigureJ plugin. 


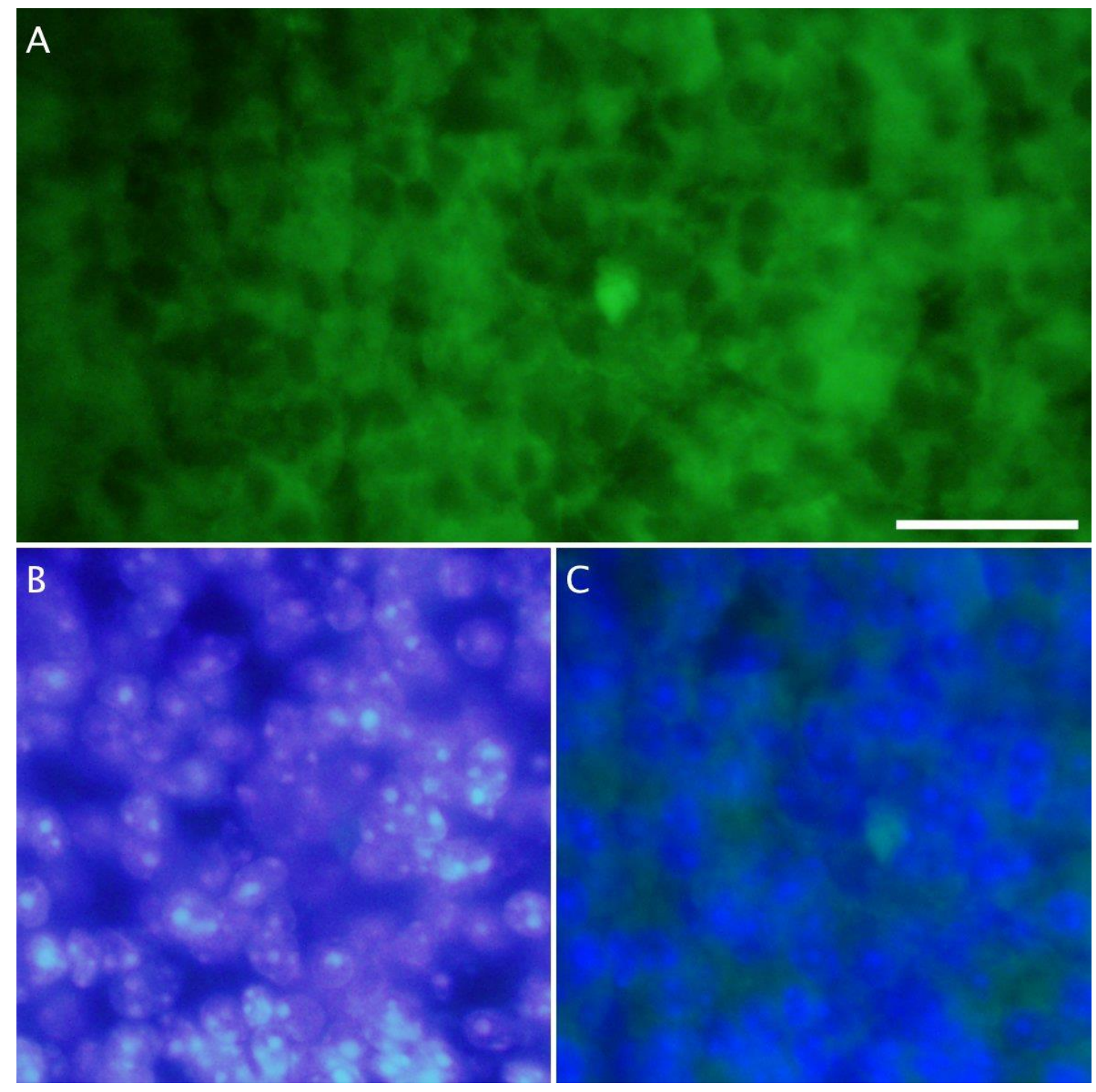

Figure 43. Representative Images of 8-OHdG and DAPI stained tumour tissue from untreated brain

A. Untreated control tumour brain tissue stained with $8-\mathrm{OHdG}$ and imaged at $100 \mathrm{x}$ objective. A large amount of background autofluorescence is visible as generalised green colour. Very little, if any 8OHdG positive punctae can be seen. B. The same field of view stained with DAPI nuclear stain. $\mathbf{C}$. The same field of view displaying an overlay of 8-OHdG and DAPI to discern any punctae that are within nuclei. The scale bar of 200 pixels corresponds to $12.9 \mu \mathrm{m}$. Images A and B were unaltered; the brightness of the composite Image $\mathrm{C}$ was increased. Produced using the ImageJ FigureJ plugin. 

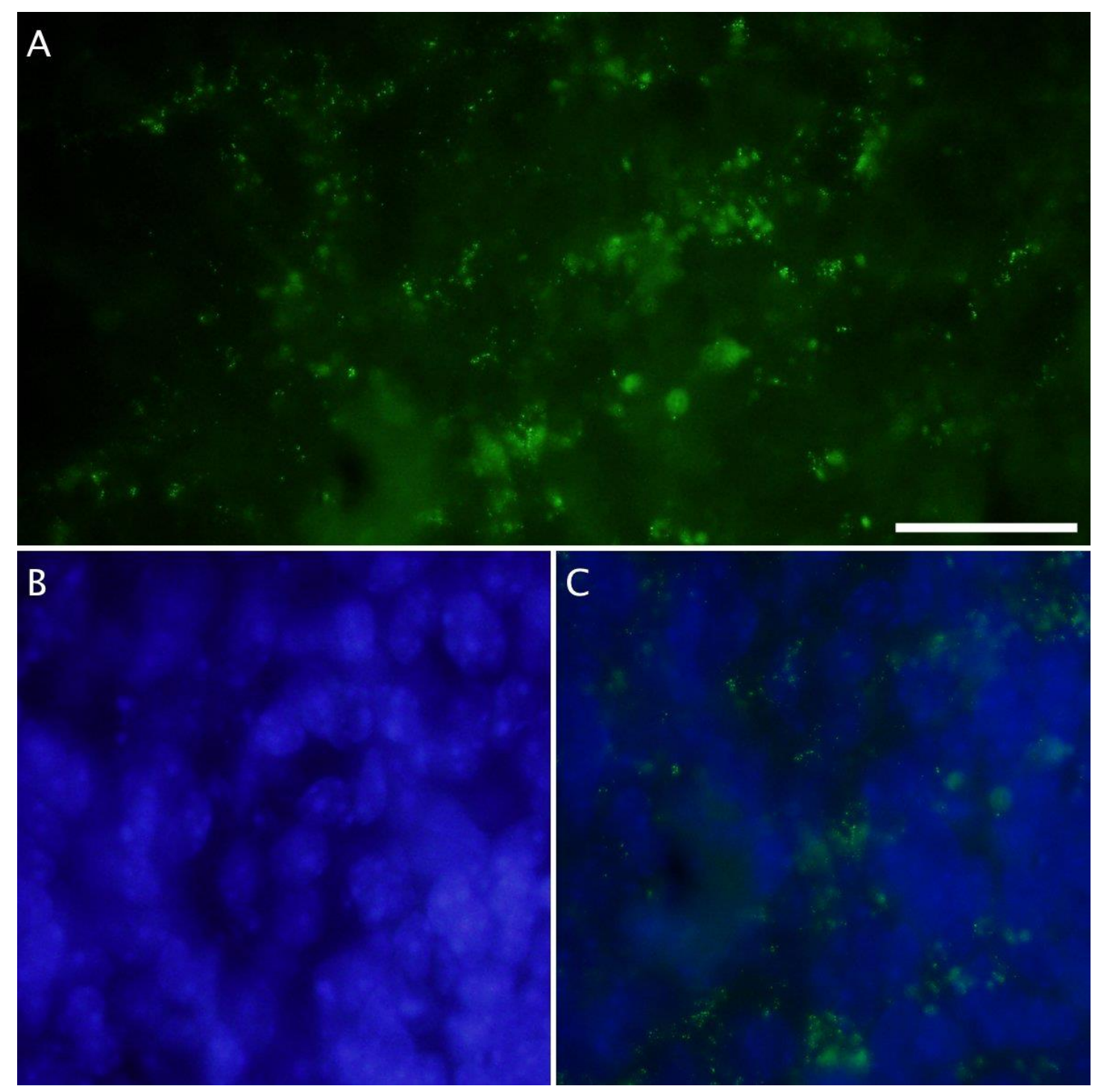

Figure 44. Representative Images of 8-OHdG and DAPI stained tumour tissue from ascorbate treated brain

A. Ascorbate treated tumour brain tissue stained with $8-\mathrm{OHdG}$ and imaged at 100x objective. Some background autofluorescence is visible as generalised green colour. Many 8-OHdG positive punctae can be seen, much more than in Figure 43. B. The same field of view stained with DAPI nuclear stain. C. The same field of view displaying an overlay of 8-OHdG and DAPI to discern punctae that are within nuclei. The scale bar of 200 pixels corresponds to $12.9 \mu \mathrm{m}$. Images A and B were unaltered; the brightness of the composite Image $\mathrm{C}$ was increased. Produced using the Image FigureJ plugin. 

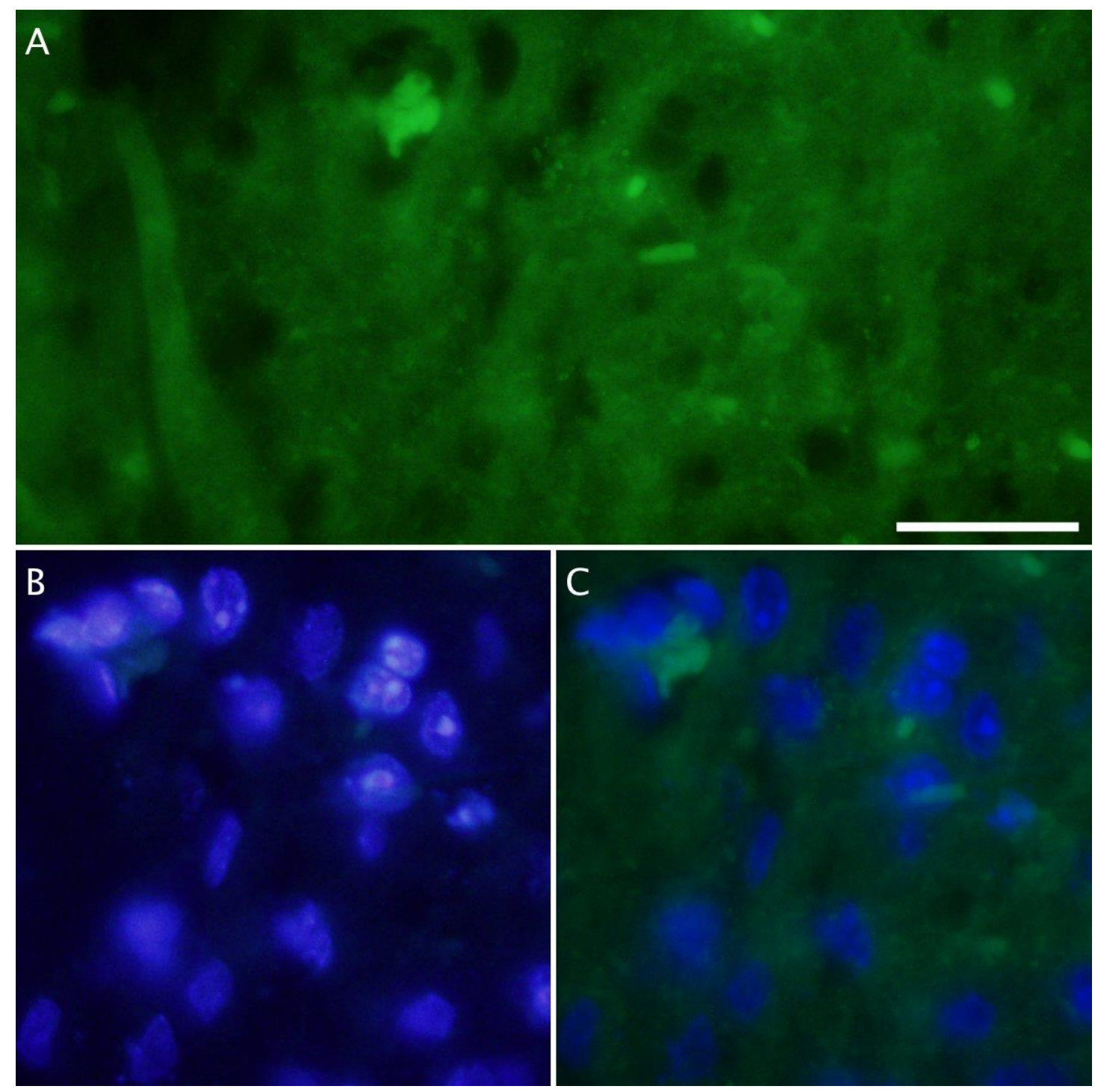

Figure 45. Representative Images of 8-OHdG and DAPI stained tumour tissue from irradiation treated brain

A. Irradiation treated tumour brain tissue stained with $8-\mathrm{OHdG}$ and imaged at $100 \mathrm{x}$ objective. A large amount of background autofluorescence is visible as generalised green colour. Some 8-OHdG positive punctae can be seen, more than control cells in Figure 43, but apparently less than ascorbate treated cells in Figure 44. B. The same field of view stained with DAPI nuclear stain. C. The same field of view displaying an overlay of $8-\mathrm{OHdG}$ and DAPI to discern punctae that are within nuclei. The scale bar of 200 pixels corresponds to $12.9 \mu \mathrm{m}$. Images A and B were unaltered; the brightness of the composite Image $\mathrm{C}$ was increased. Produced using the ImageJ FigureJ plugin. 

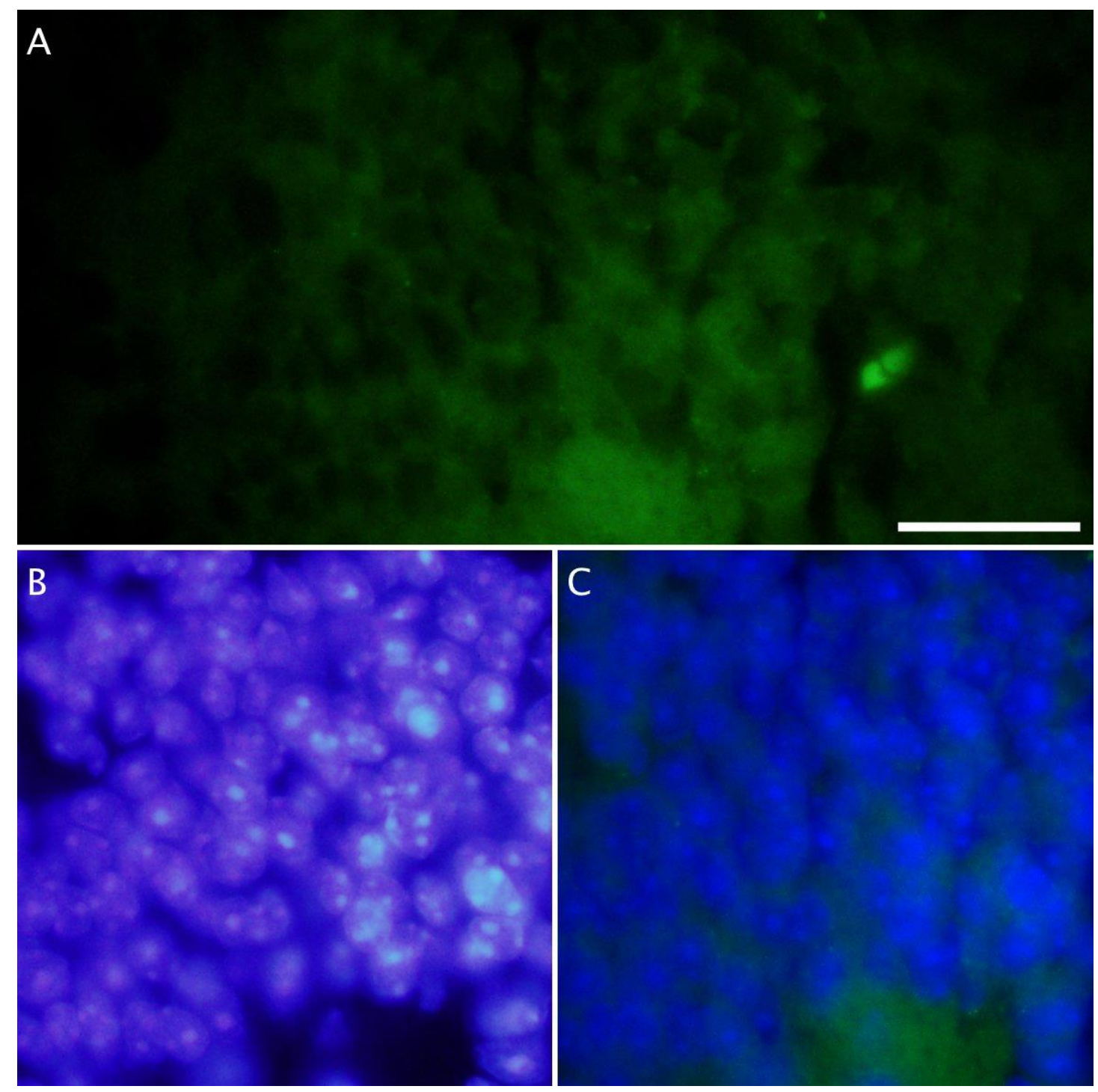

Figure 46. Representative Images of 8-OHdG and DAPI stained tumour tissue from ascorbate pre-treated, irradiated brain

A. Ascorbate pre-treatment and irradiation treated tumour brain tissue stained with 8-OHdG and imaged at 100x objective. Some background autofluorescence is visible as generalised green colour. A small amount of 8-OHdG positive punctae can be seen, less than Figure 44 or 45 . B. The same field of view stained with DAPI nuclear stain. C. The same field of view displaying an overlay of 8OHdG and DAPI to discern punctae that are within nuclei. The scale bar of 200 pixels corresponds to $12.9 \mu \mathrm{m}$. Images A and B were unaltered; the brightness of the composite Image $\mathrm{C}$ was increased. Produced using the ImageJ FigureJ plugin. 


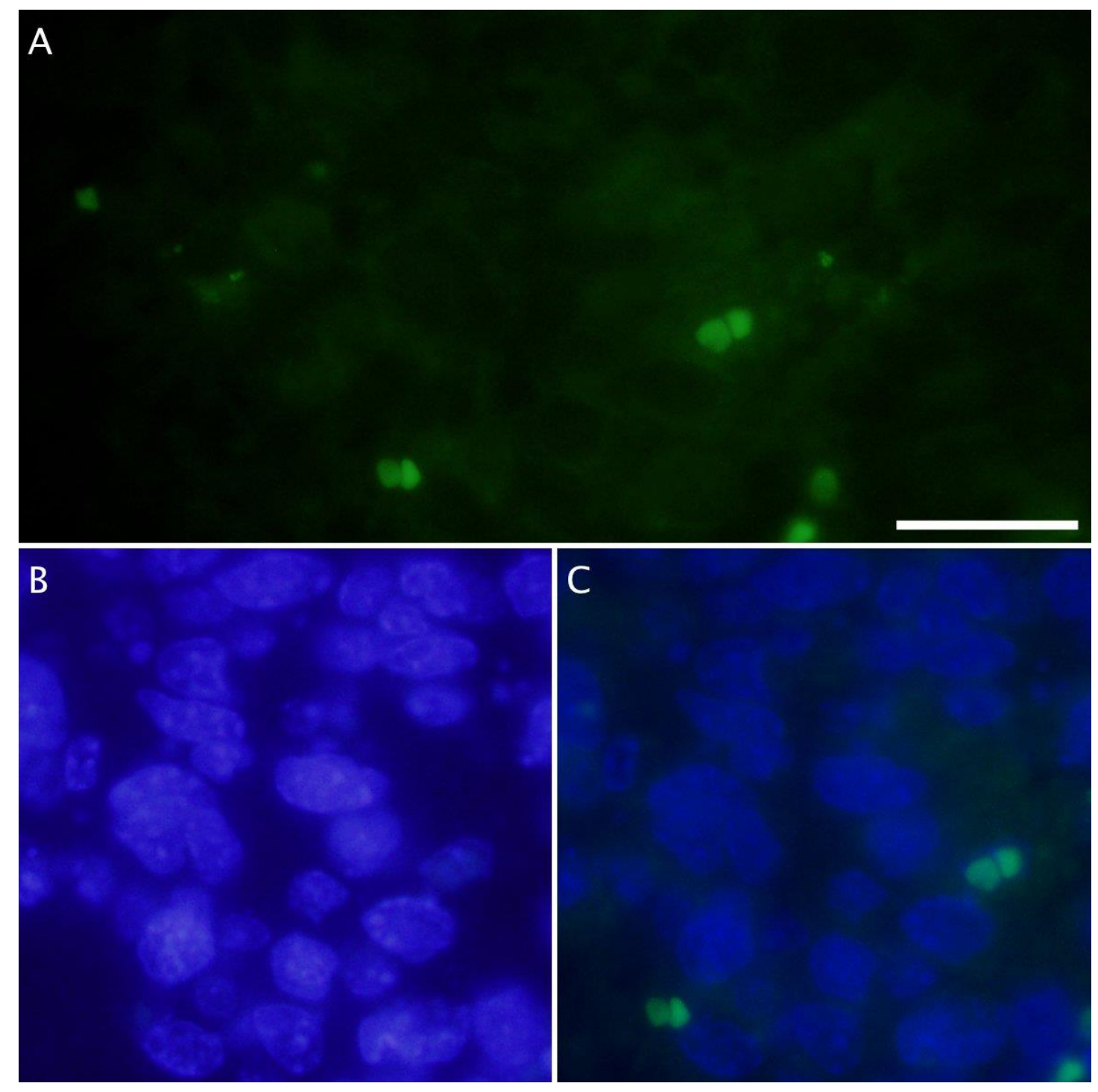

Figure 47. Representative Images of 8-OHdG and DAPI stained tumour tissue from irradiated, ascorbate post-treated brain

A. Irradiation and ascorbate post-treatment treated tumour brain tissue stained with 8-OHdG and imaged at 100x objective. A little background autofluorescence is visible as generalised green colour, although most green fluorescence is from blood and blood vessels. Very little, if any 8-OHdG positive punctae can be seen, to a relatively lower degree than Figure 44 or 45 . B. The same field of view stained with DAPI nuclear stain. C. The same field of view displaying an overlay of 8-OHdG and DAPI to discern punctae that are within nuclei. The scale bar of 200 pixels corresponds to 12.9 $\mu \mathrm{m}$. Images A and B were unaltered; the brightness of the composite Image C was increased. Produced using the ImageJ FigureJ plugin. 


\section{Chapter 4: Discussion}

GBMs are notoriously radiation and chemotherapy resistant. In this investigation it was confirmed that it is possible to radiosensitise GBM cells in vitro with ascorbate, with respect to three indicators of DNA damage, YH2AX, 8-OHdG, and DSBs. However, in vivo, ascorbate does not appear to increase the effectiveness of irradiation in causing an increase in DNA damage, as indicated by $\mathrm{\Upsilon H} 2 \mathrm{AX}$ or $8-\mathrm{OHdG}$; in fact it may potentially decrease irradiation's efficacy. A caveat to this result is the preliminary nature of in vivo results.

\section{In Vitro}

\section{YH2AX}

In vitro results from this study that show ascorbate successfully enhanced DNA damage, measured with $\mathrm{rH}_{2} \mathrm{AX}$ in GBM cells. This complements findings from other studies conducted in our lab.

Herst et al. showed that $\mathrm{\Upsilon H} 2 \mathrm{AX}$ was increased in response to $5 \mathrm{mM}$ ascorbate and 6Gy of irradiation too, although that assay was done in the primary cell line 0906 and compared with normal astrocytes, as a measure of the selectivity of treatment to cancer cells. Interestingly, in that study, ascorbate increased the proportion of $\mathrm{YH} 2 \mathrm{AX}$ positive 0906 cells to a greater degree than irradiation alone, a damage trend more similar to 8$\mathrm{OHdG}$ and the comet tail results than $\mathrm{YH} 2 \mathrm{AX}$ in this investigation.

In a more recent paper, our lab investigated the effect of radiosensitising effect of ascorbate on a panel of both cell lines and primary cells, including two of the cells used in this study, T98Gs and 1003s. That study, which used an identical treatment time of 2 hours as used here, gave comparable results.

The T98G cell line was most ascorbate resistant, with a less than 2fold increase in $\mathrm{YH} 2 \mathrm{AX}$, while irradiation and combination treatment gave higher, 3.5-fold increases. This is similar to the percent positive results seen 
here of 1.1, 3.5, and 2.5-fold percent positive increases for ascorbate, irradiation, and combination treatment respectively in T98G cells.

In contrast, 1003 results in this 2014 paper showed a consistent, just over 2-fold increase in $\mathrm{YH} 2 \mathrm{AX}$ staining 2 hours after any of the three treatments, while this investigation had the same treatments causing $2.5,2$, and 3-fold increases in $\mathrm{YH} 2 \mathrm{AX}$ for irradiation, ascorbate and combination respectively. A possible reason for the difference in results here between this investigation and that published is the further optimisation of the protocol that has been carried out since those experiments were conducted.

In Grasso et al, GL261 cells were used to investigate the radiosensitising effect of ascorbate, in a protocol very similar to that used here. $\Upsilon \mathrm{H} 2 \mathrm{AX}$ positive staining was determined, and with percent positive cells, found to be approximately 5, 6, and 9-fold greater than control fluorescence at 2 hours post treatment for irradiation, ascorbate, and combination treatments respectively. The trend differs from what is reported here (see ' $\mathrm{YH} 2 \mathrm{AX}$ '), as this study found a slightly less extreme increase in $\mathrm{YH} 2 \mathrm{AX}$ with all treatments, and opposite outcomes in response to ascorbate and irradiation alone. In contrast with Grasso et al, these $\Upsilon \mathrm{H} 2 \mathrm{AX}$ experiments report irradiation provoking more $\mathrm{\Upsilon H} 2 \mathrm{AX}$ than ascorbate, and fold increases of 3.1, 3 and 4 fold for irradiation, ascorbate, and combination. As with $\mathrm{rH} 2 \mathrm{AX}$ measurement in T98G and 1003 cells, different results may be attributable to subtle differences in protocol development between the two tests.

In conclusion, $\mathrm{rH} 2 \mathrm{AX}$ results here compared with past experiments done in our lab are broadly similar. Although sometimes variable by cell line, and subject to alterations in protocol, the overall trend stands. Ascorbate acts as a radiosensitiser and so together with irradiation causes a greater increase in $\mathrm{YH} 2 \mathrm{AX}$, and thus DNA damage, than individual treatments. 


\section{8-OHdG}

In this investigation, ascorbate enhanced DNA damage in GBM cells as indicated by the $8-\mathrm{OHdG}$ marker. As well $\mathrm{rH} 2 \mathrm{AX}, 8-\mathrm{OHdG}$ is another DNA damage marker that was used in this study. To our knowledge, 8-OHdG has not been used specifically to study the DNA damaging effects of prooxidant ascorbate in GBM cells, although it has been used in assessing the efficacy of cancer treatments, including GBM, as a prognostic marker, a test of antioxidant capacity (Gonenc, Hacisevki et al. 2012), and investigating the prooxidant effects of ascorbate in many contexts, including cancer (Valavanidis, Vlachogianni et al. 2009).

An example of surveying the effect of ascorbate's prooxidation effect with 8-OHdG is an investigation by (Poljšak, Gazdag et al. 2005), who were looking for a pre-treatment that would protect against chromium(VI) mediated damage in a yeast model. Interestingly, in vitro, ascorbate acted as an antioxidant in the absence of chromium, but as a concentration dependent prooxidant in its presence, as measured by 8OHdG. Yet in vivo, ascorbate decreased cytotoxicity and acted in its radical scavenging role, an effect the authors ascribed to increased cytosol reduction and better scavenging abilities. This result mirrors that found in this investigation with opposite in vitro and in vivo results, and validates 8OHdG as an accurate and suitable marker for detecting ascorbate's prooxidant activity.

8-OHdG has also been successfully used in investigating prognostic outcomes in cancer studies. One example studied its correlation with survival and tumour grade in ovarian cancer, finding that high incidence of 8-OHdG in serum and tumour tissue highly predicted tumour grade and was negatively correlated with survival (Pylväs, Puistola et al. 2011).

Another study was in glioma (Lian, Zhang et al. 2014), and examined the relationship between 8-OHdG marking and autophagy, as indicated by microtubule-associated protein 1 light chain-3B (LC3B). Tests used both ex vivo tumours and were validated with the U87 cell, a standard 
GBM cell line. As with the ovarian cancer study, tumour grade was positively correlated with levels of 8-OHdG. Grade and 8-OHdG also predicted increased LC3B, an indicator of autophagy. Interestingly, prevention of autophagy with an inhibitor caused a strong increase in ROS production.

These two prognostic cancer study results are inverse to that hypothesised in this investigation, where an increase in $8-\mathrm{OHdG}$ correlates with DNA damage and is so inferred to predict the effectiveness of treatment, rather than the effectiveness of tumour malignancy. It may be, however, that below a certain threshold of $8-\mathrm{OHdG}$, the marker is associated with increased tumour mutation and aggressiveness, while above that threshold 8-OHdG indicates excessive DNA damage that the cell is unable to repair and will cause cell death.

Although $\Upsilon \mathrm{H} 2 \mathrm{AX}$ as a marker of DNA damage, specifically DSBs, had been used extensively in our lab prior to this investigation, $8-\mathrm{OHdG}$ as a marker of oxidative stress was newly introduced for this study. Thus past results cannot be exactly compared, yet nonetheless interesting parallels can be drawn. For instance, although measurement of ROS load was not ventured here, there are interesting trends between ROS load, as investigated in Castro et al, and 8-OHdG levels in response to the same doses of ascorbate, at least in T98G and 1003 cells. This comparison is consistent with ascorbate's mechanism of action as a ROS mediated DNA damager.

In that paper, T98Gs displayed an approximately 2-fold difference in ROS production in response to ascorbate versus hydrogen peroxide, with. $5 \mathrm{mM}$ of ascorbate producing double the ROS load to $50 \mu \mathrm{M}$ of hydrogen peroxide. In a similar way, T98Gs in this investigation had greater susceptibility to ascorbate than to hydrogen peroxide as judged by the appearance of 8-OHdG; ascorbate only had a 5-fold increase in 8-OHdG, while hydrogen peroxide caused a 3.5-fold increase. 
Similarly, ROS load results followed the same trend as 8-OHdG occurrence in 1003 cells, and the opposite trend to the T98G cells. Castro et al. showed that 1003 cells increased ROS more in response to hydrogen peroxide than ascorbate, although the difference was only in the region of $20 \%$. Likewise, $8-\mathrm{OHdG}$ measurements of 1003 cells here found that hydrogen peroxide caused greater incidence of the marker compared with ascorbate, although in this case, the treatment difference was closer to 2fold. These similarities can be interpreted as corroboration of the antioxidant capacity and specific vulnerability to ascorbate and hydrogen peroxide in these cells.

\section{Comet Tail Assay}

The comet tail assay, as a measure of DNA strand breaks, is used in a variety of applications, such as investigating the toxicity of environmental substances, basic research into DNA damaging agents, clinical responses, and even as a test of the suitability of sperm for IVF (in vitro fertilisation) treatments. It has also begun to be investigated as a prognosis marker for cancer.

Like the other two in vitro assays used here, the comet tail assay has also been applied to investigation of ascorbate's prooxidant capacity and ability to induce DNA strand breaks. Using the comet tail assay, Ullah et al. (2010) also showed the prooxidant effect of ascorbate in lymphocytes, and that it could be inhibited by the addition of iron and copper chelators, highlighting the importance of these metals. The authors proposed therefore that differential availability of metal catalysts in cancer as opposed to regular cells, may be a mechanism of selectivity seen in some experiments. The comet tail assay is known to be applicable to all eukaryotic cells, yet specific reporting of ascorbate's prooxidant effect from this paper and others (Duarte, Almeida et al. 2007; Yen, Duh et al. 2002) with this assay is a validation of its use here. 
There are also studies that have used the comet tail assay to assess cancer treatments potential to cause DNA damage, including those for GBM (Chakravarti, Erkkinen et al. 2006). For instance, Lu et al. (2010) published an investigation into the effect of a cytotoxic agent danthron in GBM 8401 cells, that found comet assay tail lengths were significantly lengthened in response. Another study (Hardee, Marciscano et al. 2012) used neurosphere cultures of GL261 cells to measure alterations in DNA damage from irradiation by prior treatment with a transforming growth factor- $\beta$ (TGF $\beta$ ) inhibitor. That study found that treating GBM cells with inhibitor enhanced the effect of fractionation alone in increasing DNA damage, which points to the DNA damage response as a key mediator of radioresistance in GBM.

As for our own lab's experiments, the comet tail was newly established during this investigation, and so cannot be directly contrasted with our previous results. However, some comparisons can be made with previous studies involving $\mathrm{YH} 2 \mathrm{AX}$. This comparison is relevant due to the similarity in reported function as indicators of DSBs.

One interesting example of difference is cell repair after exposure to ascorbate. As previously described, an optimisation experiment for the comet tail assay was an ascorbate treatment time course including measurement of DSB repair up to 24 hours post 1-hour ascorbate exposure. In that assay, it was found that by 8 hours after treatment, the number of DSBs had regressed to approaching control levels (see 'Ascorbate Time Course'). This varies with results displayed in (Castro, McConnell et al. 2014) which reported many cell lines with impaired DSB repair even at 24 hours post-ascorbate, based solely on the resolution of $\mathrm{YH}^{\mathrm{A}} \mathrm{AX}$. However, in that paper the GL261 response was not measured, which, as a murine GBM cell line may possess different cell repair abilities. Furthermore, as mentioned, differences between the assays prevent direct contrast. 
An additional assay that could have been carried out in this study is the comet tail assay on ex vivo samples of brain tumour and normal tissue. Although here the comet tail assay was only performed on in vitro cells, use of the assay on ex vivo tissue is not uncommon (Tice, Agurell et al. 2000) and would only require homogenisation of tissue into individual cells.

Another alteration to this assay that may have been informative is the common version involving an alkaline DNA unwinding step, which measures the degree of single strand breaks (SSBs). That assay was attempted once, although was not optimised (data not shown).

The neutral version of the comet tail assay was focussed on for two reasons. Firstly, as previously mentioned, DSBs are considered the most cytotoxic DNA alteration, and more likely to cause cell death than the relatively easier repaired SSBs. Secondly, in an alkaline assay, cell cycle status contributes to the comet parameters, since single strand breaks arise as a normal part of cell replication (Fairbairn and O'Neill 1995). Only undertaking DSB measurements should have limited this cell cycle effect.

As mentioned, the comet tail assay was newly established in our lab by the author, and despite optimisation may benefit from further alterations to the protocol. In the experiments presented here, cell nuclei comets were all analysed manually with a comet tail plugin for the ImageJ software, and so will contain inherently more variability than if analysed automatically. Although more sophisticated and automated programs are available, they are expensive and require specific microscope imaging software, so were not used.

In Vivo

\section{Immunofluorescence}

This report's results regarding immunofluorescence assays of tumour brain tissue, although preliminary, complement findings made in our own lab. Those findings, which contradicted our other findings on the 
radiosensitising role of ascorbate in vitro, originally provoked this investigation, and are corroborated by the initial details given here.

Grasso et al. (2014) used a GL261 murine model of GBM to investigate the potential radiosensitising role of ascorbate that had been evident in vitro. Unexpectedly, as explained in 'Introduction', tumourbearing mice that were treated with both 4.5 Gy of irradiation and $1 \mathrm{~g} / \mathrm{kg}$ of ascorbate had significantly worse survival than those mice treated with ascorbate alone. These data suggested that instead of making the tumour more sensitive to radiation, ascorbate in fact protected the tissue from radiation damage.

If ascorbate does in fact act as radio-protector in vivo, that would mesh with results presented here. Overall survival was not the focus of this study, but the amount of DNA damage correlates with the efficacy of treatment and therefore clinical relevance. It was found in this investigation (see 'Tumour Immunofluorescence') that although both ascorbate and irradiation individually caused a noticeable increase in both $\Upsilon \mathrm{H} 2 \mathrm{AX}$ and 8OHdG, indicating DNA damage, that fluorescence was strongly limited by combination treatments. Further work should be carried out to fully optimise the protocol detailed and develop a method of quantification that was not possible here.

\section{Confounding Factors}

As mentioned in the introduction, there are a variety of ideas surrounding the mechanism of ascorbate's action. Many in vitro studies investigate the prooxidant mechanism that causes DNA damage. However, there are a variety of other factors at play, such as hypoxia, that could complicate ascorbate's function and causing these conflicting results in vitro and in vivo results.

Environmental conditions, or the cancer microenvironment, are pivotal to the effect of a given treatment on cancer. Generally, and in this 
study, in vitro cultures are kept at $20 \%$ oxygen and have their media changed regularly to ensure a constant supply of nutrients. Cells are also regularly passaged to guarantee sufficient space.

These controlled conditions are in stark contrast to that found in the in vivo environment, where oxygen levels can vary between 5 and less than $1 \%$ (Herst et al). Like many other solid forms of cancer, GBM contains hypoxic regions within the tumour mass. This hypoxia is the result of rapid cell growth and disorganised, subpar angiogenesis. Areas of hypoxia influence tumour outcomes in many ways. For example, hypoxic regions are better able to resist radiation because of their requirement for oxygen and and chemotherapy for the lack of functioning blood vessel access.

In relation to the effect of ascorbate on tumours, a factor that should be considered is ascorbate's influence on HIF1a, as described in 'Ascorbate as a Co-factor'. Although some experiments have shown ascorbate supplementation decreased HIF1 activity, the process may be more complicated. For example, there is some evidence that $\mathrm{H}_{2} \mathrm{O}_{2}$ can inhibit HIF1 hydroxylases (Kuiper and Vissers 2014), which would thus increase the activity of HIF1 and so cause induction of pro-survival factors. Ascorbate can create hydrogen peroxide by the mechanism outlined in Eq 7/8 (see 'Ascorbate as a Prooxidant'), and so could potentially contribute to this effect, in an opposing action to its established behaviour as a metal reducing, HIF1 inactivating co-factor.

Moreover, recent evidence has detailed how ascorbate's oxidation reaction is strongly dependent on oxygen, such that when samples were flushed with argon to limit oxygen, peak creation of ascorbate radicals was nearly 3-fold lower than oxygen-containing solutions (Boatright 2015).

A potential treatment development that might be investigated is to increase the oxygen concentration in tumours, by methods such as hyperbaric oxygen chambers (Moen and Stuhr 2012). If combined with ascorbate in cancer therapy, increased oxygen could lessen hypoxia's 
confounding effects on ascorbate's mechanism and lead to determination of a factor that causes these in vitro and in vivo differences. Initial investigations of the effect of supplemental oxygen in vivo models prior to clinical practice could be undertaken by a research lab with those particular facilities.

Another possibility is that ascorbate is not even being converted to hydrogen peroxide at all in vivo. Because ascorbate in its oxidised form is taken up by SVCT transporters, cells that upregulate the transporters may be importing ascorbate before the conversion has even taken place, a conversion that happens in the extracellular space. This may be causing ascorbate to act as a traditional antioxidant and so limit the cytotoxicity of irradiation (Grasso, Fabre et al. 2014; McConnell and Herst 2014). Further investigation could measure the expression of transporters at time points after inoculation of murine models with GBM, or correlate the expression of transporters in ex vivo primary tumour cells with eventual survival. This would fill in current gaps in our knowledge of ascorbate's transport in the particular context of GBM.

Of course it is also possible that ascorbate has its effect in a complex multifaceted way that includes these factors and more. The conflicting results, even between in vivo studies, suggests that much more investigation is needed in order to work out the subtleties of ascorbate's variable effects in the animal context.

\section{Selectivity}

Some have suggested that not only does ascorbate cause DNA damage and cell death in cells, but that this effect is specific to cancerous cells. This would be of great clinical significance if correct, as it would allow for high-dose ascorbate to cause cytotoxicity in cancerous cells while avoiding death to regular healthy cells. Many cancer therapies cause damage to healthy cells, but that disadvantage is considered to be outweighed by the effective cytotoxicity to cancer cells. If ascorbate could be shown to have cytotoxic effect against cancer while acting in a non- 
harmful way to normal cells, that would give a strong advantage to its use. There are reasons to accept this claim of specificity, although our lab group has not seen evidence of it thus far (Castro, McConnell et al. 2014).

For example, cancerous cells already have significant DNA anomalies and mutations that have caused the initial uncontrolled replication. Strategies to detoxify in response to oxidative stress will also be compromised. Secondly, cancer cells' higher glucose requirements for excessive growth cause up-regulation of GLUT transporters and are more likely to take up the oxidised ascorbate DHA. DHA in high doses has also shown to be cytotoxic. Thirdly, cancerous cells have 10-1000 times less catalase than regular healthy cells (Benade, Howard et al. 1969), and so will be less able to inhibit cytotoxicity caused by ascorbate-created hydrogen peroxide. And finally, cancer cells may contain higher levels of metals available to catalase prooxidant reactions (Ullah, Khan et al. 2010).

These hypothetical reasons seem to have been borne out with actual evidence of selectivity in some in vitro (Benade, Howard et al. 1969; Bram, Froussard et al. 1980; Noto, Taper et al. 1989) and in vivo (Pierson and Meadows 1983; Tsao 1991; Varga and Airoldi 1983) studies. Although, significantly, these studies were not carried out in GBM or brain cells, and so are not directly comparable the particular GBM situation.

In the face of this, it would have been informative to undertake all tests in parallel with non-malignant brain cells to test any potential specificity between tumorigenic and non-tumorigenic cells. However, this would have required purchase of a new cell line or technically difficult immortalisation of primary astrocytes for comparison.

For the in vivo experiments, non-tumour tissue acted as that normal cell equivalent, and initial results allowed some assessment of whether ascorbate's action was selective to cancerous cells. In experiments carried out for this investigation, no differences could be seen in the presence of $\mathrm{rH} 2 \mathrm{AX}$ and $8-\mathrm{OHdG}$ punctae in response to ascorbate in tumour compared 
with non-tumour tissue (images not shown). Although clearly if ascorbate is not confirmed to function at all as a prooxidant cancer therapy in vivo, then the subject of selectivity is insignificant.

\section{The Role of Metals}

A key consideration in the DNA damaging effects of ascorbate is the catalytic role of transition metals. There is some question over whether there even enough catalytically available metal in vivo to cause the effects we expect to see, or even whether metals are as important in this case as expected (Gonzalez 2014; Halliwell and Gutteridge 1986). If they are, differences in metal concentrations are a possible explanation for the different effects seen in vitro and in vivo in this and other experiments.

The data produced in this study supports a significant role for metals in the ascorbate reaction, as displayed in the large difference in production of DNA DSBs in response to ascorbate when treated in PBS versus in DMEM media (see 'Combination Treatments').

Carr and Frei's (Carr and Frei 1999) meta-analysis of cell culture studies that measured markers of DNA, protein, and lipid oxidative damage, concluded that in general, ascorbate performed in type and acted as antioxidant. However, in studies with added metal, ascorbate transformed into a pro-oxidant and increased markers of stress such as single stranded breaks in the single cell gel electrophoresis (See 'Comet Tail Assay').

Yet it is dangerous to unquestioningly extrapolate from in vitro to in vivo metallic conditions. Although cell culture media contains sufficient free metals to catalyse the required Fenton/Haber-Weiss reaction, those same levels of metal might not be present in vivo, preventing the full potential of the reaction. Some have pointed out that although the body is filled with trace metals, very little of these are catalytically available, due to binding to molecules such as ferritin (Carr and Frei 1999; Gonzalez 2014). 
But if this is found to be the case, it may be straightforward to fix by supplementing the diets of patients with available metals (McCarty and Contreras 2014). For example, Tsao found the mice that ate an ionic copper supplemented diet had improved anti-proliferative effects by ascorbate on their human mammary xenografts (Tsao 1991).

Interestingly, several studies have investigated a possible DNA damaging effect of ascorbate without mediation by transition metals, and have found cytotoxicity in metal-free cultures. Nappi et al. (Nappi and Vass 2000) showed that ascorbate and hydrogen peroxide alone were capable of producing hydroxyl radicals within minutes, and more effectively than ascorbate-metal or metal-hydrogen peroxide complexes. In this case, ascorbate directly oxidises hydrogen peroxide to form water and the hydroxyl radical.

$$
\mathrm{AA}+\mathrm{H}_{2} \mathrm{O}_{2} \longrightarrow \mathrm{AA}+\mathrm{H}_{2} \mathrm{O}+\mathrm{OH}
$$

However, they did not use physiological concentrations of reactants in that study, thus its biological impact is unknown. It may be that this reaction is negligible compared to the creation of hydroxyl radicals in processes that do make use of catalytic metals. Certainly, the level of free metals seems to correlate with the degree of ROS creation. For example, in vitro, DMEM creates more hydrogen peroxide then RPMI, thought to be due to the extra $0.25 \mu \mathrm{M}$ of $\mathrm{Fe}(\mathrm{NO} 3) 3$ (Clement, Ramalingam et al. 2001).

A further interesting experiment could have been to test the $\mathrm{rH} 2 \mathrm{AX}$ and 8-OHdG markers for DNA damage with flow cytometry in metal free conditions. This could have been achieved with the same conditions as used in the comet tail assay, and treatment of cells in culture while in PBS rather than regular DMEM media. Another interesting possibility would have been to investigate metal supplementation of a subset of ascorbate treated mice, in order to ascertain any potential difference in tumour DNA damage outcomes. 
The importance of metal also brings up a caveat to all experiments outlined here. Cell culture media age can affect the amount of trace metals present and so available during assays undertaken in media. In the assays presented here, cell culture DMEM and RPMI was made up in $500 \mathrm{~mL}$ volumes and used within a calendar month. It may be possible that this difference in media age at the time of any experiment played a part in varying rapidity of the creation of hydrogen peroxide from ascorbate and so altered results.

\section{Limitations}

To be expected, there are a number of caveats and limitations to bear in mind when assessing this investigation, in addition to those previously mentioned.

The unique nature of the brain environment, enclosed by the blood brain barrier, with a high oxygen requirement and the particular characteristics of brain cells, prevents direct translation of data from papers that study cancers not in this environment. Unfortunately, much of the evidence that these experiments used as the foundation were not brain cancer or GBM, but were instead predominantly pancreatic, breast, or prostate cancers. In the same way, specific data from this study may not directly translate for scientists studying the effect of ascorbate in non-brain cancers.

Another limitation is the inability to directly translate information derived from vivo models, such as those studied here, to the human context. Potential contrasts must be considered carefully before undertaking human clinical trials. Inevitably, there are large differences between artificially inoculated cancer in mice, to naturally occurring GBM in humans, which displays its own particular genetics. In fact, the differential cell response to identical treatments as seen here, is evidence that GL261s are by no means indistinguishable from human GBM. 
Treatment application will also differ, complicating efforts to translate relevance. As mentioned, ascorbate given by parental infusion is considered a representative model of human intravenous injection. Yet, pharmacokinetic studies by Chen et al. (2007) showed that intravenous and intraperitoneal-delivered ascorbate concentrations, although they follow the same trend, are not entirely identical. Intravenous application, that used in human studies, causes higher ascorbate concentrations in the early time points than intraperitoneal, the method used in most rodent studies. Several millimoles' difference was reported in peak plasma levels ( $3 \mathrm{mM}$ compared with $8 \mathrm{mM}$ plasma concentration) between the two methods. Because the subtleties of ascorbate's effect at a given concentration has not been fully elucidated, it may eventually be determined that this difference makes a difference in ascorbate's prooxidant effect.

In this study, as with many other cancer research investigations, normal C57BL6 mice were used as murine models. However, in the context of ascorbate, they may not be the ideal treatment simulator. Mice, unlike humans and a small number of other organisms, can synthesise their own ascorbate, instead of having to consume it in the diet (see 'Ascorbate as a Cofactor'). Although mice naturally do not synthesise the molecule to nearly the concentration expected to induce prooxidant effect, it is an extra complication with potential implications to results. Unfortunately, guinea pigs or GULO knockout mice were unavailable for research here.

Interpretation of pharmacokinetic tests in healthy individuals, and how to relate this information to cancer patients is another caveat. Most pharmacokinetic research is based on healthy individuals, while studies in cancer patients generally do not directly measure the ascorbate to serum concentration correlation. It may be the case that cancer patients have altered absorption such that general pharmacokinetic conclusions are not relatable to their condition ( $\mathrm{Li}$ and Schellhorn 2007). It may even be found that cancer patients, with their high requirements for ascorbic acid, deplete the systemic concentration such that remaining ascorbate acts as a traditional antioxidant, and serves to radio-protect cancer. 
Because some trials have found that low doses of ascorbate increase the proliferation of cells (Riordan, Riordan et al. 1995), the specific concentration at which ascorbate transforms from an antioxidant to a prooxidant, and the conditions that influence this, should be carefully investigated. Despite widespread use of the molecule by alternative healthcare practitioners, it is not clear under precisely which conditions (concentration, tissue, amount of metals, transport) where the balance of its function shifts.

It may also be that the nature of human serum itself is a limitation in being able to extrapolate from in vitro to in vivo data. Riordan et al. reported that introducing $20 \%$ human serum to culture medium attenuated the cytotoxic effects of ascorbate.

\section{Future Experiments}

Due to the time constraints, there are extra assays that may have been informative in this investigation, but were not possible, in addition to those mentioned previously in this discussion. They may be researched in the future in our lab.

For instance, although already carried out in our lab on some measures of ascorbate's effect, it would have been helpful to attempt a reversal of ascorbate's prooxidant capacity with the hydrogen peroxide mitigating catalase, especially considering addition of hydrogen peroxide itself did not exactly correspond to ascorbate effect in the 8-OHdG assay. Catalase provokes the decomposition of $\mathrm{H}_{2} \mathrm{O}_{2}$ to water and oxygen. If catalase abrogated DNA damaging effect in the specific assays performed here, it would confirm peroxide mediation of this effect in a larger variety of types of damage.

A further possibility might have been to investigate potential mechanisms of ascorbate's cytotoxicity, such as the role of necrosis, apoptosis, autophagy or chromatin collapse, or some possible combination 
of pathways for cell death. Thus far, some mechanisms have been investigated, but often contradict, due to some as yet unknown factors. It may also be that ascorbate's cytotoxicity mechanism varies depending on ascorbate concentration, or whether it functions in conjunction with another DNA damaging agent.

For in vivo application, it could have been informative to attempt different patterns of treatment other than short term (treatment on the day of harvest) versus longer term (treatment 24 hours prior to harvest). Although simplification in mouse studies is necessary for practicality, other regimens may have better represented human study. For example, a truly long term experiment may have been more representative of the treatment that human cancer patients go through, and include several treatments over the course of weeks.

Ultimately, the most important next step in this area of research is to confirm ascorbate's activity in vivo, where clinical relevance comes to the fore. If it is ineffective, it should be determined by which mechanism the in vitro efficacy is destroyed, and then if ineffectiveness is common under all or only some conditions, such as low oxygen.

\section{Conclusion}

In conclusion, GBM is a highly malignant brain cancer that has thus far eluded development of effective treatment. Traditional therapies such as radiation and chemotherapy fail to sufficiently extend lifespan, as such, investigations into potential therapy sensitizers are on-going. Ascorbate has long been proposed as a biologically plausible anti-cancer molecule due to its many faceted roles in anti-oxidation, catalysis, and prooxidation, and although in vitro results are plentiful, credible and consistent in vivo studies are lacking. Conflicting in vitro and in vivo outcomes from our lab 
originally provoked this investigation, which aimed to focus on varieties of DNA damage in both contexts, and confirm or falsify the discrepancy.

In vitro radiosensitisation evidence was validated by two assays, flow cytometry and the neutral comet tail assay, that measured three complementary markers designed to determine DNA damage; $\Upsilon \mathrm{H} 2 \mathrm{AX}$, a DNA damage-induced histone phosphorylation involved in the DNA damage response; $8-\mathrm{OHdG}$, a hydroxylation modification to the DNA base guanine that indicates oxidative damage, and DSBs, the most severe variant of DNA damage, and most likely to cause cell death. These assays were carried out on GL261s; a murine model of GBM, T98Gs; a human immortalised GBM cell line, and 1003s; a primary cell derived from ex vivo tumour tissue.

Although results varied somewhat between these cell lines and assays, the broad in vitro trend was an increase in all markers of DNA damage in response to either $5 \mathrm{mM}$ of ascorbate or $6 \mathrm{~Gy}$ of irradiation, but an even larger increase when cells received both treatments, confirmation of ascorbate's radiosensitising function.

In vivo experiments established intracranial GL261 model of GBM in C57BL6 mice and treated them with ascorbate, irradiation or both treatments before brain tumour and non-tumour tissue was harvested and underwent immunofluorescence. Two of the markers used on in vitro cells, rH2AX and 8-OHdG, were assessed in images of both cancerous and normal brain, in brains of mice that were given each of the treatments.

Although not fully optimised, the overall trend of in vivo data seemed to indicate that ascorbate does not supplement the effectiveness of irradiation in causing an increase in DNA damage, as indicated by $\Upsilon \mathrm{H} 2 \mathrm{AX}$ or 8-OHdG. In fact, it may potentially decrease irradiation's efficacy. Results were similar in cancerous and healthy tissue, and the sequence of 
ascorbate pre or post irradiation did not appear to make an observable difference.

Therefore, experiments detailed here show a credible radiosensitisation of GBM cells in vitro by ascorbate, as well as a potential radioprotective role for ascorbate in vivo. If confirmed, ascorbate treatment should be discontinued in patients also receiving radiation therapy, for it may lead, tragically, to shorter survival in an already deadly disease. 


\section{Bibliography}

Adamson, C., O. O. Kanu, A. I. Mehta, C. Di, N. Lin, A. K. Matox, and D. D. Bigner (2009). "Glioblastoma multiforme: a review of where we have been and where we are going." Expert Opinion Investigational Drugs 18(8): 1061-1083.

Agus, D. B., S. s. Gambhir, W. M. Pardridge, C. Spielholz, J. Baselga, J. C. Vera and D. W. Golde (1997). "Vitamin Crosses the Blood-Brain Barier in the Oxidised Form Through the Glucose Transporters." The American Society for Clinical Investigation 100(11): 2842-2848.

Alifieris, C. and D. T. Trafalis (2015). "Glioblastoma multiforme: Pathogenesis and treatment." Pharmacology and Therapeutics 152: 63-82. Allen, B. G., Z. A. Sibenaller, J. J. Cullen, G. R. Buettner, T. L. Carlisle, M. C. Smith, S. A. Walsh, J. E. Bayouth, M. TenNapel and D. R. Spitz (2012). "Pharmacological Ascorbate Enhances Chemo-Radio-Sensitization in Brain and Lung Cancer." Free Radical Biology and Medicine 53(2):S39.

Ausman, J. I., W. R. Shapiro and D. P. Rall (1970). "Studies on the Chemotherapy of Experimental Brain Development of an Experimental Model." Cancer Research 30: 2394-2400.

Australian Government Department of Health and Ageing National Health and Medical Research Council and New Zealand Ministry of Health (2006). "Nutrient Reference Values for Australia and New Zealand." K. Baghurst., Ball, G. F. M. (2008). Vitamin C. Vitamins, Blackwell Publishing Ltd: 393420.

Banhegyi, G., L. Braun, M. Csala, F. Puskas and J. Mandl (1997). "Ascorbate Metabolism and its Regulation in Animals." Free Radical Biology and Medicine 23(5): 793-803.

Barnum, C. P., M. S. Scheller and N. P. Herman (1964). "Acute Effect of Gamma Radiation on DNA Synthesis." Cancer Research 24: 1155-1158. Beels, L., K. Bacher, D. De Wolf, J. Werbrouck and H. Thierens (2009). "gamma-H2AX foci as a biomarker for patient $\mathrm{X}$-ray exposure in pediatric cardiac catheterization: are we underestimating radiation risks?" Circulation 120(19): 1903-1909.

Benade, L., T. Howard and D. Burk (1969). "Synergistic Killing of Ehrlich Ascites Carcinoma Cells by Ascorbate and 3-Amino-1,2,4,-triazole." Oncology 23: 33-43.

Bendich, A. and R. J. Deckelbaum (2015). Preventative Nutrition. Bhat, S. H., A. S. Azmi, S. Hanif and S. M. Hadi (2006). "Ascorbic acid mobilizes endogenous copper in human peripheral lymphocytes leading to oxidative DNA breakage: a putative mechanism for anticancer properties." The International Journal of Biochemistry \& Cell Biology 38(12): 20742081.

Bielski, B. H., H. W. Richter and P. C. Chan (1975). "Some properties of the ascorbate free radical." Annals of the New York Academy of Sciences 258: 231-237. 
Bjelakovic, G., D. Nikolova, R. G. Simonetti and C. Gluud (2004).

"Antioxidant supplements for prevention of gastrointestinal cancers: a systematic review and meta-analysis." The Lancet 364(9441): 1219-1228.

Block, G. (1991). "Epidemiologic evidence regarding vitamin C and cancer." American Journal of Clinical Nutrition 54: 1310S-1314S.

Bo, Y., Y. Lu, Y. Zhao, E. Zhao, L. Yuan, W. Lu, L. Cui and Q. Lu (2015). "Association between dietary vitamin $C$ intake and risk of esophageal cancer: A dose-response meta-analysis." International Journal of Cancer 138(8): 1843-1850.

Boatright, W. L. (2015). "Oxygen Dependency of One-Electron Reactions Generating Ascorbate Radicals and Hydrogen Peroxide from Ascorbic Acid." Food Chemistry 196:1361-1367.

Bondy, M. L., M. E. Scheurer, B. Malmer, J. S. Barnholtz-Sloan, F. G. Davis, D. Il'yasova, C. Kruchko, B. J. McCarthy, P. Rajaraman, J. A. Schwartzbaum, S. Sadetzki, B. Schlehofer, T. Tihan, J. L. Wiemels, M. Wrensch, P. A. Buffler and C. Brain Tumor Epidemiology (2008). "Brain tumor epidemiology: consensus from the Brain Tumor Epidemiology Consortium." Cancer 113/7 Suppl): 1953-1968.

Bram, S., P. Froussard, M. Guichard, C. Jasmin, Y. Augery, F. Sinoussi-Barre and W. Wray (1980). "Vitamin C preferential toxicity for malignant melanoma cells." Nature 284: 629-631.

Broadley, K. W., M. K. Hunn, K. J. Farrand, K. M. Price, C. Grasso, R. J. Miller, I. F. Hermans and M. J. McConnell (2011). "Side population is not necessary or sufficient for a cancer stem cell phenotype in glioblastoma multiforme." Stem Cells 29(3): 452-461.

Brumbaugh, K. M., D. M. Otterness, C. Geisen, V. Oliveira, J. Brognard, X. Li, F. Lejeune, R. S. Tibbetts, L. E. Maquat and R. T. Abraham (2004). "The mRNA Surveillance Protein hSMG-1 Functions in Genotoxic Stress Response Pathways in Mammalian Cells." Molecular Cell 14(5): 585-598. Buettner, G. R. and B. A. Jurkiewicz (1996). "Catalytic Metals, Ascorbate and Free Radicals: Combinations to Avoid." Radiation Research 145(5): 532-541.

Bureau International des Poids et Mesures. (2014). "SI Brochure: The International System of Units (SI)." 8th edition. Retrieved 13 December, 2015, from http://www.bipm.org/en/publications/si-brochure/.

Burgi, S., A. Seuwen, R. Keist, J. Vom Berg, J. Grandjean and M. Rudin (2014). "In vivo imaging of hypoxia-inducible factor regulation in a subcutaneous and orthotopic GL261 glioma tumor model using a reporter gene assay." Molecular Imaging 13.

Burns, J. J. (1957). "Missing Step in Man, Monkey and Guinea Pig required for the Biosynthesis of L-Ascorbic Acid." Nature 180(4585): 553.

Cameron, E., A. Campbell and T. Jack (1975). "The orthomolecular treatment of cancer. III. Reticulum cell sarcoma: double complete regression induced by high-dose ascorbic acid therapy." ChemicoBiological Interactions 11(5): 387-393.

Cameron, E. and L. Pauling (1976). "Supplemental ascorbate in the supportive treatment of cancer: Prolongation of survival times in terminal 
human cancer." Proceedings of the National Academy of Sciences of the United States of America 73(10): 3685-3689.

Cameron, E. and L. Pauling (1979). "Ascorbate and Cancer." Proceedings of the American Philosophical Society 123(2): 117-123.

Cameron, E., L. Pauling and B. Leibovitz (1979). "Ascorbic acid and cancer: a review." Cancer Research 39(3): 663-681.

Campbell, E. J., M. C. Vissers, S. Bozonet, A. Dyer, B. A. Robinson and G. U. Dachs (2015). "Restoring physiological levels of ascorbate slows tumor growth and moderates HIF-1 pathway activity in Gulo(-/-) mice." Cancer Medicine 4(2): 303-314.

Carpenter, K. J. (1988). The History of Scurvy and Vitamin C. Cambridge: Cambridge University Press.

Carr, A. and B. Frei (1999). "Does vitamin C act as a pro-oxidant under physiological conditions?" The Federation of American Societies for Experimental Biology Journal 13(9): 1007-1024.

Castro, M. L., M. J. McConnell and P. M. Herst (2014). "Radiosensitisation by pharmacological ascorbate in glioblastoma multiforme cells, human glial cells, and HUVECs depends on their antioxidant and DNA repair capabilities and is not cancer specific." Free Radical Biology \& Medicine 74: 200-209.

Chakravarti, A., M. G. Erkkinen, U. Nestler, R. Stupp, M. Mehta, K. Aldape, M. R. Gilbert, P. M. Black and J. S. Loeffler (2006). "TemozolomideMediated Radiation Enhancement in Glioblastoma: A Report on Underlying Mechanisms." Clinical Cancer Research 12(15): 4738-4746. Chen, P., J. Stone, G. Sullivan, J. A. Drisko and Q. Chen (2011). "Anti-cancer effect of pharmacologic ascorbate and its interaction with supplementary parenteral glutathione in preclinical cancer models." Free Radical Biology \& Medicine 51(3): 681-687.

Chen, Q., M. G. Espey, A. Y. Sun, J. H. Lee, M. C. Krishna, E. Shacter, P. L. Choyke, C. Pooput, K. L. Kirk, G. R. Buettner and M. Levine (2008). "Ascorbate in pharmacologic concentrations selectively generates ascorbate radical and hydrogen peroxide in extracellular fluid in vivo." Proceedings of the National Academy of Sciences 104(21): 8749-8754. Chinot, O. L. (2009). "High grade astrocytomas." In: R. P. Lisak, D. D. Truong, W. M. Carroll and R. Bhidayasiri (eds): International Neurology: A Clinical Approach. Oxford: Blackwell Publishing: 506-509.

Ciriello, G., M. L. Miller, B. A. Aksoy, Y. Senbabaoglu, N. Schultz and C. Sander (2013). "Emerging landscape of oncogenic signatures across human cancers." Nature Genetics 45(10): 1127-1133.

Clement, M. V., J. Ramalingam, L. H. Long and B. Halliwell (2001). "The in vitro cytotoxicity of ascorbate depends on the culture medium used to perform the assay and involves hydrogen peroxide." Antioxidants and Redox Signaling 3(1): 157-163.

Colavitti, R., G. Pani, B. Bedogni, R. Anzevino, S. Borrello, J. Waltenberger and T. Galeotti (2002). "Reactive oxygen species as downstream mediators of angiogenic signaling by vascular endothelial growth factor receptor2/KDR." Journal of Biological Chemistry 277(5): 3101-3108. 
Comroe, J. H. (1978). "Experimental studies designed to evaluate the management of patients with incurable cancer." Proceedings of the National Academy of Sciences of the United States of America 75(9): 4543. Cooke, M. S., M. D. Evans, M. Dizdaroglu and J. Lunec (2003). "Oxidative DNA damage: mechanisms, mutation, and disease." The Federation of American Societies for Experimental Biology Journal 17(10): 1195-1214. Crawford, T. C. (1982). "Synthesis of L-Ascorbic Acid." 200: 1-36. Creagan, E. T., C. G. Moertel, J. R. O'Fallon, A. J. Schutt, M. J. O'Connell, J. Rubin and S. Frytak (1979). "Failure of high-dose vitamin C (ascorbic acid) therapy to benefit patients with advanced cancer. A controlled trial." New England Journal of Medicine 301(13): 687-690.

Crespo, I., A. L. Vital, M. Gonzalez-Tablas, C. Patino Mdel, A. Otero, M. C. Lopes, C. de Oliveira, P. Domingues, A. Orfao and M. D. Tabernero (2015). "Molecular and Genomic Alterations in Glioblastoma Multiforme." The American Journal of Pathology 185(7): 1820-1833.

Dickson, K. M., C. B. Gustafson, J. I. Young, S. Zuchner and G. Wang (2013). "Ascorbate-induced generation of 5-hydroxymethylcytosine is unaffected by varying levels of iron and 2-oxoglutarate." Biochemical and Biophysical Research Communications 439(4): 522-527.

Division of Life Sciences (2011). A Comprehensive approach to Life Science. Tokyo, The University of Tokyo.

Domitrović, R. (2006). "Vitamin C in disease prevention and therapy." Biochemia Medica 16(2): 107-125.

Driessens, N., S. Versteyhe, C. Ghaddhab, A. Burniat, X. De Deken, J. Van Sande, J. E. Dumont, F. Miot and B. Corvilain (2009). "Hydrogen peroxide induces DNA single- and double-strand breaks in thyroid cells and is therefore a potential mutagen for this organ." Endocrine-Related Cancer 16(3): 845-856.

Du, J., S. M. Martin, M. Levine, B. A. Wagner, G. R. Buettner, S. H. Wang, A. F. Taghiyev, C. Du, C. M. Knudson and J. J. Cullen (2010). "Mechanisms of ascorbate-induced cytotoxicity in pancreatic cancer." Clinical Cancer Research 16(2): 509-520.

Duarte, T. L., G. M. Almeida and G. D. Jones (2007). "Investigation of the role of extracellular $\mathrm{H} 2 \mathrm{O} 2$ and transition metal ions in the genotoxic action of ascorbic acid in cell culture models." Toxicology Letters 170(1): 57-65. Dutta, A., R. Gautam, S. Chatterjee, F. Ariese, S. K. Sikdar and S. Umapathy (2015). "Ascorbate Protects Neurons against Oxidative Stress: A Raman Microspectroscopic Study." ACS Chemical Neuroscience 6(11): 1794-1801. Elmore, A. R. (2005). "Final report of the safety assessment of L-Ascorbic Acid, Calcium Ascorbate, Magnesium Ascorbate, Magnesium Ascorbyl Phosphate, Sodium Ascorbate, and Sodium Ascorbyl Phosphate as used in cosmetics." International Journal of Toxicology 24 Suppl 2: 51-111.

Emerit, J., M. Edeas and F. Bricaire (2004). "Neurodegenerative diseases and oxidative stress." Biomedicine \& Pharmacotherapy 58(1): 39-46. Englard, S. and S. Seifter (1986). "The biochemical functions of ascorbic acid." Annual Review of Nutrition 6: 365-406. 
Eriksson, D. and T. Stigbrand (2010). "Radiation-induced cell death mechanisms." Tumour Biology 31(4): 363-372.

Esteller, M., J. Garcia-Foncillas, E. Andion, S. N. Goodman, O. F. Hidalgo, V. Vanaclocha, S B. Baylin, and J. G. Herman (2000). "Inactivation of the DNArepair gene MGMT and the clinical response of gliomas to alkylating agents." New England Journal of Medicine 343(19): 1350-1354.

Fairbairn, D. W. and K. L. O'Neill (1995). "Necrotic DNA degradation mimics apoptotic nucleosomal fragmentation comet tail length." In Vitro Cellular and Developmental Biology - Animal 31(3): 171-173.

Fatma, N., E. Kubo, P. Sharma, D. R. Beier and D. P. Singh (2005). "Impaired homeostasis and phenotypic abnormalities in Prdx6-//-mice lens epithelial cells by reactive oxygen species: increased expression and activation of TGF[beta]." Cell Death \& Differentiation 12(7): 734-750.

Ficz, G., M. R. Branco, S. Seisenberger, F. Santos, F. Krueger, T. A. Hore, C. J. Marques, S. Andrews and W. Reik (2011). "Dynamic regulation of 5hydroxymethylcytosine in mouse ES cells and during differentiation." Nature 473(7347): 398-402.

Fukui, M., N. Yamabe, H. J. Choi, K. Polireddy, Q. Chen and B. T. Zhu (2015). "Mechanism of Ascorbate-Induced Cell Death in Human Pancreatic Cancer Cells: Role of Bcl-2, Beclin 1 and Autophagy." Planta Medica 81(10): 838846.

Gao, P., H. Zhang, R. Dinavahi, F. Li, Y. Xiang, V. Raman, Z. M. Bhujwalla, D. W. Felsher, L. Cheng, J. Pevsner, L. A. Lee, G. L. Semenza and C. V. Dang (2007). "HIF-dependent antitumorigenic effect of antioxidants in vivo." Cancer Cell 12(3): 230-238.

Garcia-Krauss, A., L. Ferrada, A. Astuya, K. Salazar, P. Cisternas, F. Martinez, E. Ramirez and F. Nualart (2015). "Dehydroascorbic Acid Promotes Cell Death in Neurons Under Oxidative Stress: a Protective Role for Astrocytes." Molecular Neurobiology.

Gaziano, J. M., R. J. Glynn, W. G. Christen, T. Kurth, C. Belanger, J. MacFadyen, V. Bubes, J. E. Manson, H. D. Sesso and J. E. Buring (2009). "Vitamins $\mathrm{E}$ and $\mathrm{C}$ in the Prevention of Prostate and Total Cancer in Men." JAMA 301(1).

Gey, K. F. (1998). "Vitamins E plus $C$ and interacting conutrients required for optimal health." BioFactors 7: 113-174.

Gonenc, A., A. Hacisevki, S. Aslan, M. Torun and B. Simsek (2012). "Increased oxidative DNA damage and impaired antioxidant defense system in patients with gastrointestinal cancer." European Journal of Internal Medicine 23(4): 350-354.

Gonzalez, M. J. (2014). New Insights on Vitamin C and Cancer. New York, Springer-Verlag.

Grasso, C., M. S. Fabre, S. V. Collis, M. L. Castro, C. S. Field, N. Schleich, M. J. McConnell and P. M. Herst (2014). "Pharmacological doses of daily ascorbate protect tumors from radiation damage after a single dose of radiation in an intracranial mouse glioma model." Frontiers in Oncology 4: 356. 
Greenberg, H. S., W. F. Chandler and H. M. Sandler (1999). Brain Tumors. New York: Oxford University Press.

Haber, F. and J. Weiss (1934). "The Catalytic Decomposition of Hydrogen

Peroxide by Iron Salts." Proceedings of the Royal Society of London A:

Mathematical, Physical and Engineering Sciences 147(861): 332-351.

Halliwell, B. (2001). "Vitamin C and genomic stability." Mutation Research 475(1-2): 29-35.

Halliwell, B. and J. M. Gutteridge (1986). "Oxygen free radicals and iron in relation to biology and medicine: some problems and concepts." Archives of Biochemistry and Biophysics 246(2): 501-514.

Hardee, M. E., A. E. Marciscano, C. M. Medina-Ramirez, D. Zagzag, A.

Narayana, S. M. Lonning and M. H. Barcellos-Hoff (2012). "Resistance of glioblastoma initiating cells to radiation mediated by the tumor microenvironment can be abolished by inhibiting transforming growth factor- $\beta$ (TGF $\beta$ )." Cancer Research 72(16): 4119-4129.

Head, K. A. (1998). "Ascorbic Acid in the Prevention and Treatment of Cancer." Alternative Medicine Review 3(3): 174-186.

Hemila, H. and E. Chalker (2013). "Vitamin C for preventing and treating the common cold." Cochrane Database Syst Rev 1: Cd000980.

Herst, P. M., K. W. Broadley, J. L. Harper and M. J. McConnell (2012).

"Pharmacological concentrations of ascorbate radiosensitize glioblastoma multiforme primary cells by increasing oxidative DNA damage and inhibiting G2/M arrest." Free Radical Biology \& Medicine 52(8): 1486-1493. Hoffmann, H., J. Hogel and G. Speit (2005). "The effect of smoking on DNA effects in the comet assay: a meta-analysis." Mutagenesis 20(6): 455-466. Huang, Z., L. Cheng, O. A. Guryanova, Q. Wu and S. Bao (2010). "Cancer stem cells in glioblastoma-molecular signaling and therapeutic targeting." Protein and Cell 1(7): 638-655.

Hunn, M. K., K. J. Farrand, K. W. Broadley, R. Weinkove, P. Ferguson, R. J. Miller, C. S. Field, T. Petersen, M. J. McConnell and I. F. Hermans (2012). "Vaccination with irradiated tumor cells pulsed with an adjuvant that stimulates NKT cells is an effective treatment for glioma." Clinical Cancer Research 18(23): 6446-6459.

Huycke, M. M. (2003). "Single-Cell Gel Electrophoresis or Comet Assay of Intestinal Epithelial Cells Using Manual Scoring and Ridit Analysis." In:

Hensley, K., and Floyd, R. A. (eds): Methods in Biological Oxidative Stress. Totowa: Humana Press: pgs 101-108.

Ivashkevich, A., C. E. Redon, A. J. Nakamura, R. F. Martin and O. A. Martin (2012). "Use of the gamma-H2AX assay to monitor DNA damage and repair in translational cancer research." Cancer Letters 327(1-2): 123-133.

Jackson, S. P. and J. Bartek (2009). "The DNA-damage response in human biology and disease." Nature 461(7267): 1071-1078.

Kallner, A., D. Hartmann and D. Hornig (1979). "Steady-state turnover and body pool of ascorbic acid in man." The American Journal of Clinical Nutrition 32(3): 530-539.

Karcher, S., H. H. Steiner, R. Ahmadi, S. Zoubaa, G. Vasvari, H. Bauer, A. Unterberg and C. Herold-Mende (2006). "Different angiogenic phenotypes 
in primary and secondary glioblastomas." International Journal of Cancer 118(9): 2182-2189.

Klaunig, J. E., Y. Xu, J. S. Isenberg, S. Bachowski, K. L. Kolaja, J. Jiang, D. E. Stevenson and E. F. Walborg, Jr. (1998). "The role of oxidative stress in chemical carcinogenesis." Environmental Health Perspectives 106(Suppl 1): 289-295.

Klungland, A., I. Rosewell, S. Hollenbach, E. Larsen, G. Daly, B. Epe, E. Seeberg, T. Lindahl and D. E. Barnes (1999). "Accumulation of premutagenic DNA lesions in mice defective in removal of oxidative base damage." Proceedings of the National Academy of Sciences 96(23): 1330013305.

Kuiper, C. (2012). The Role of Ascorbate in the Regulation of HIF-1 in Cancer Cells. Doctor of Philosophy, University of Otago, Christchurch. Kuiper, C., G. U. Dachs, M. J. Currie and M. C. Vissers (2014). "Intracellular ascorbate enhances hypoxia-inducible factor (HIF)-hydroxylase activity and preferentially suppresses the HIF-1 transcriptional response." Free Radical Biology \& Medicine 69: 308-317.

Kuiper, C., G. U. Dachs, D. Munn, M. J. Currie, B. A. Robinson, J. F. Pearson and M. C. Vissers (2014). "Increased Tumor Ascorbate is Associated with Extended Disease-Free Survival and Decreased Hypoxia-Inducible Factor-1 Activation in Human Colorectal Cancer." Frontiers in Oncology 4: 10.

Kuiper, C. and M. C. Vissers (2014). "Ascorbate as a co-factor for fe- and 2oxoglutarate dependent dioxygenases: physiological activity in tumor growth and progression." Frontiers in Oncology 4(359): 1-11.

Kuiper, C., M. C. Vissers and K. O. Hicks (2014). "Pharmacokinetic modeling of ascorbate diffusion through normal and tumor tissue." Free Radical Biology \& Medicine 77(2014): 340-352.

Kunsch, C. and R. M. Medford (1999). "Oxidative Stress as a Regulator of Gene Expression in the Vasculature." Circulation Research 85(8): 753-766.

Kurbacher, C. M., U. Wagner, B. Kolster, P. E. Andreotti, D. Krebs and H. W. Bruckner (1996). "Ascorbic acid (vitamin C) improves the antineoplastic activity of doxorubicin, cisplatin, and paclitaxel in human breast carcinoma cells in vitro." Cancer Letters 103(2): 183-189.

Laurenzi, V. D., G. Melino, I. Savini, M. Annicchiarico-Petruzzelli, A. FinazziAgro and L. Avigliano (1995). "Cell Death by Oxidative Stress and Ascorbic Acid Regeneration in Human Neuroectodermal Cell Lines." European Journal of Cancer 31A(4): 463-466.

Lee, Y. W., H. J. Cho, W. H. Lee and W. E. Sonntag (2012). "Whole Brain Radiation-Induced Cognitive Impairment: Pathophysiological Mechanisms and Therapeutic Targets." Biomolecules \& Therapeutics 20(4): 357-370. Legendre, C. and E. Garcion (2015). "Iron metabolism: a double-edged sword in the resistance of glioblastoma to therapies." Trends in Endocrinology and Metabolism 26(6): 322-331.

Leibel, S. A., C. B. Scott and T. F. Pajak (1991). "The Management of Malignant Gliomas with Radiation Therapy: Therapeutic Results and Research Strategies." Seminars in Radiation Oncology 1(1): 32-49. 
Levine, M., C. Conry-Cantilena, Y. Wang, R. W. Welch, P. W. Washiko, K. R. Dhariwal, J. B. Park, A. Lazarev, J. F. Gramlich, J. King and L. R. Cantilena (1996). "Vitamin C pharmacokinetics in healthy volunteers: Evidence for a recommended dietary allowance." Proceedings of the National Academy of Sciences 93: 3704-3709.

Levine, M., S. J. Padayatty and M. G. Espey (2011). "Vitamin C: a concentration-function approach yields pharmacology and therapeutic discoveries." Advances in Nutrition 2(2): 78-88.

Li, M., X. Chen, P. Wang and F. Ma (2011). "Ascorbic Acid Accumulation and Expression of Genes Involved in Its Biosynthesis and Recycling in Developing Apple Fruit." Journal of the American Society for Horticultural Science 136(4): 231-238.

Li, Y. and H. E. Schellhorn (2007). "New Developments and Novel Therapeutic Perspectives for Vitamin C." The Journal of Nutrition 137(10): 2171-2184.

Lian, M., X. Zhang, H. Wang, H. Liu, W. Chen and S. Guo (2014). "Increased 8-hydroxydeoxyguanosine in high-grade gliomas is associated with activation of autophagy." International Journal of Neuroscience 124(12): 926-934.

Liao, W., M. A. McNutt and W. G. Zhu (2009). "The comet assay: a sensitive method for detecting DNA damage in individual cells." Methods 48(1): 4653.

Lin, J., N. R. Cook, C. Albert, E. Zaharris, J. M. Gaziano, M. Van Denburgh, J. E. Buring and J. E. Manson (2009). "Vitamins $C$ and $E$ and beta carotene supplementation and cancer risk: a randomized controlled trial." Journal of the National Cancer Institute 101(1): 14-23.

Lindahl, T. and R. D. Wood (1999). "Quality control by DNA repair." Science 286(5446): 1897-1905.

Linster, C. L. and E. Van Schaftingen (2007). "Vitamin C - Biosynthesis, recycling and degradation in mammals." The FEBS Journal 274(1): 1-22. Liu, L.-Z., X.-W. Hu, C. Xia, J. He, Q. Zhou, X. Shi, J. Fang and B.-H. Jiang (2006). "Reactive oxygen species regulate epidermal growth factorinduced vascular endothelial growth factor and hypoxia-inducible factor$1 \alpha$ expression through activation of AKT and P70S6K1 in human ovarian cancer cells." Free Radical Biology and Medicine 41(10): 1521-1533.

Lo, Y. Y. and T. F. Cruz (1995). "Involvement of reactive oxygen species in cytokine and growth factor induction of c-fos expression in chondrocytes." The Journal of Biological Chemistry 270(20): 11727-11730.

Loria, C. M., M. J. Klag, L. E. Caulfield and P. K. Whelton (2000). "Vitamin C status and mortality in US adults." American Journal of Clinical Nutrition 72(1): 139-145.

Louis, D. N., H. Ohgaki, O. D. Wiestler, W. K. Cavenee, P. C. Burger, A. Jouvet, B. W. Scheithauer and P. Kleihues (2007). "The 2007 WHO Classification of Tumours of the Central Nervous System." Acta Neuropathologica 114(2): 97-109.

Lu, H. F., T. Y. Lai, T. C. Hsia, Y. J. Tang, J. S. Yang, J. H. Chiang, C. C. Lu, C. M. Liu, H. L. Wang and J. G. Chung (2010). "Danthron induces DNA damage 
and inhibits DNA repair gene expressions in GBM 8401 human brain glioblastoma multiforms cells." Neurochemical Research 35(7): 1105-1110. Lunet, N., C. Valbuena, A. L. Vieira, C. Lopes, C. Lopes, L. David, F. Carneiro and $\mathrm{H}$. Barros (2007). "Fruit and vegetable consumption and gastric cancer by location and histological type: case-control and meta-analysis."

European Journal of Cancer Prevention 16(4): 312-327.

Lushchak, V. I. (2014). "Free radicals, reactive oxygen species, oxidative stress and its classification." Chemico-Biological Interactions 224(2014): 164-175.

Pylväs M., U. Puistola, L. Laatio, S. Kauppila and P. Karihtala (2011).

"Elevated Serum 8-OHdG Is Associated with Poor Prognosis in Epithelial Ovarian Cancer." Anticancer Research 31(4): 1411-1415.

Ma, Y., J. Chapman, M. Levine, K. Polireddy, J. Drisko and Q. Chen (2014). "High-Dose Parenteral Ascorbate Enhanced Chemosensitivity of Ovarian Cancer and Reduced Toxicity of Chemotherapy." Science Translational Medicine 6(222): 222ra18.

Mariani, C. J., A. Vasanthakumar, J. Madzo, A. Yesilkanal, T. Bhagat, Y. Yu, S. Bhattacharyya, R. H. Wenger, S. L. Cohn, J. Nanduri, A. Verma, N. R. Prabhakar and L. A. Godley (2014). "TET1-mediated hydroxymethylation facilitates hypoxic gene induction in neuroblastoma." Cell Reports 7(5): 1343-1352.

Mathios, D. (2016). "Therapeutic administration of IL-15 superagonist complex ALT-803 leads to long-term survival and durable antitumor immune response in a murine glioblastoma model." International Journal of Cancer 138(1): 187-194.

McCarty, M. F. and F. Contreras (2014). "Increasing Superoxide Production and the Labile Iron Pool in Tumor Cells may Sensitize Them to Extracellular Ascorbate." Frontiers in Oncology 4(249): 1-9.

McConnell, M. J. and P. M. Herst (2014). "Ascorbate Combination Therapy: New Tool in the Anticancer Toolbox?" Science Translational Medicine 6(222): $222 \mathrm{fs} 6$.

McCormick, W. J. (1952). "Ascorbic Acid as a Chemotherapeutic Agent." Archives of Pediatrics 69(4): 151-155.

McCormick, W. J. (1954). "Cancer: the preconditioning factor in pathogenesis; a new etiologic approach." Archives of Pediatrics 71(10): 313-322.

McCormick, W. J. (1959). "Cancer: A collagen Disease, Secondary to a Nutritional Deficiency?" Archives of Pediatrics 76(4): 166-171. Meredith, M. E. and J. M. May (2013). "Regulation of embryonic neurotransmitter and tyrosine hydroxylase protein levels by ascorbic acid." Brain Research 1539: 7-14.

Mikirova, N., J. Casciari, N. Riordan and R. Hunninghake (2013). "Clinical experience with intravenous administration of ascorbic acid: achievable levels in blood for different states of inflammation and disease in cancer patients." Journal of Translational Medicine 11(2013): 191.

Minor, E. A., B. L. Court, J. I. Young and G. Wang (2013). "Ascorbate induces ten-eleven translocation (Tet) methylcytosine dioxygenase- 
mediated generation of 5-hydroxymethylcytosine." The Journal of Biological Chemistry 288(19): 13669-13674.

Miura, K. and A. C. Green (2015). "Dietary Antioxidants and Melanoma: Evidence from Cohort and Intervention Studies." Nutrition and Cancer 67(6): 867-876.

Miyatake, S., R. L. Martuza and S. D. Rabkin (1997). "Defective herpes simplex virus vectors expressing thymidine kinase for the treatment of malignant glioma." Cancer Gene Therapy 4(4): 222-228.

Moen, I. and L. E. B. Stuhr (2012). "Hyperbaric oxygen therapy and cancer-a review." Targeted Oncology 7(4): 233-242.

Moertel, C. G., T. R. Fleming, E. T. Creagan, J. Rubin, M. J. O'Connell and M. M. Ames (1985). "High-Dose Vitamin C versus Placebo in the Treatment of Patients with Advanced Cancer Who Have Had No Prior Chemotherapy." New England Journal of Medicine 312(3): 137-141.

Morris, J. R., C. Boutell, M. Keppler, R. Densham, D. Weekes, A. Alamshah, L. Butler, Y. Galanty, L. Pangon, T. Kiuchi, T. Ng and E. Solomon (2009). "The SUMO modification pathway is involved in the BRCA1 response to genotoxic stress." Nature 462(7275): 886-890.

Myllylä, R., E. R. Kuutti-Savolainen and K. I. Kivirikko (1978). "The role of ascorbate in the prolyl hydroxylase reaction." Biochemical and Biophysical Research Communications 83(2): 441-448.

Naidu, K. A. (2003). "Vitamin C in human health and disease is still a mystery? An overview." Nutrition Journal 2(7).

Naidu, K. A., J. L. Tang, K. A. Naidu, L. D. Prockop, S. V. Nicosia and D. Coppola (2001). "Antiproliferative and apoptotic effect of ascorbyl stearate in human glioblastoma multiforme cells: modulation of insulin-like growth factor-I receptor (IGF-IR) expression." Journal of Neuro-Oncology 54(1): 15-22.

Nakamura, M., T. Watanabe, U. Klangby, C. Asker, K. Wiman, Y. Yonekawa, P. Kleihues and H. Ohgaki (2001). "p14ARF Deletion and Methylation in Genetic Pathways to Glioblastomas." Brain Pathology 11(2): 159-168. Nakamura, M., T. Watanabe, Y. Yonekawa, P. Kleihues and H. Ohgaki (2001). "Promoter methylation of the DNA repair gene MGMT in astrocytomas is frequently associated with $\mathrm{G}: \mathrm{C} \rightarrow \mathrm{A}: \mathrm{T}$ mutations of the TP53 tumor suppressor gene." Carcinogenesis 22(10): 1715-1719.

Nappi, A. J. and E. Vass (2000). "Hydroxyl Radical Production by Ascorbate and Hydrogen Peroxide." Neurotoxicity Research 2(4): 343-355.

Newcomb, E. W. and D. Zagzag (2009). "The Murine GL261 Glioma Experimental Model to Assess Novel Brain Tumor Treatments." In: B. A. Teicher (ed): CNS Cancer: models, markers, prognostic factors, targets and therapeutic approaches. Dordrecht: Humana Press: pgs 227-241.

Newton, H. M., D. B. Morgan, C. J. Schorah and R. P. Hullin (1983).

"Relation between intake and plasma concentration of vitamin $C$ in elderly women." British Medical Journal (Clinical research ed.) 287(6403): 14291429.

Nishimura, S. (2006). "8-Hydroxyguanine: From its discovery in 1983 to the present status." Proceedings of the Japan Academy 82(4): 127-141. 
Noctor, G. and C. H. Foyer (1998). "Ascorbate and Glutathione: Keeping Active Oxygen Under Control." Annual Review of Plant Physiology and Plant Molecular Biology 49: 249-279.

Noto, V., H. S. Taper, J. Yi-Hua, J. Janssens, J. Bonte and W. de Loecker (1989). "Effects of Sodium Ascorbate (Vitamin C) and 2-Methyl-1,4Naphthoquinone (Vitamin K3) Treatment on Human Tumor Cell Growth In Vitro." Cancer 63(5): 901-906.

Ohgaki, H., P. Dessen, B. Jourde, S. Horstmann, T. Nishikawa, P. L. Di Patre, C. Burkhard, D. Schüler, N. M. Probst-Hensch, P. C. Maiorka, N. Baeza, P. Pisani, Y. Yonekawa, M. G. Yasargil, U. M. Lütolf and P. Kleihues (2004). "Genetic Pathways to Glioblastoma: A Population-Based Study." Cancer Research 64(19): 6892-6899.

Ohgaki, H. and P. Kleihues (2005). "Population-Based Studies on Incidence, Survival Rates, and Genetic Alterations in Astrocytic and Oligodendroglial Gliomas." Journal of Neuropathology and Experimental Neurology 64(6): 479-489.

Ohgaki, H. and P. Kleihues (2013). "The definition of primary and secondary glioblastoma." Clinical Cancer Research 19(4): 764-772.

Okorukwu, O. N. and K. P. Vercruysse (2003). "Effects of ascorbic acid and analogs on the activity of testicular hyaluronidase and hyaluronan lyase on hyaluronan." Journal of Enzyme Inhibition and Medicinal Chemistry 18(4): 377-382.

Olson, A. L. and J. E. Pessin (1996). "Structure, function, and regulation of the mammalian facilitative glucose transporter gene family." Annual Review of Nutrition 16: 235-256.

Omuro, A. and L. M. DeAngelis (2013). "Glioblastoma and other malignant gliomas: a clinical review." JAMA 310(17): 1842-1850.

Ostling, O. and K. J. Johanson (1984). "Microelectrophoretic study of radiation-induced DNA damages in individual mammalian cells."

Biochemical and Biophysical Research Communications 123(1): 291-298.

Padayatty, S. J. and M. Levine (2001). "New insights into the physiology and pharmacology of vitamin C." Canadian Medical Association Journal 164(3): 353-355.

Padayatty, S. J., A. Y. Sun, Q. Chen, M. G. Espey, J. Drisko and M. Levine (2010). "Vitamin C: intravenous use by complementary and alternative medicine practitioners and adverse effects." PLOS ONE 5(7:e11414): 1-8. Padayatty, S. J., H. Sun, Y. Wang, H. D. Riordan, S. M. Hewitt, A. Katz, R. A. Wesley and M. Levine (2004). "Vitamin C Pharmacokinetics: Implications for Oral and Intravenous Use." Annals of Internal Medicine 140(7): 533537.

Palmer, H. J. and K. E. Paulson (1997). "Reactive oxygen species and antioxidants in signal transduction and gene expression." Nutrition Reviews 55(10): 353-361.

Parrow, N. L., J. A. Leshin and M. Levine (2013). "Parenteral ascorbate as a cancer therapeutic: a reassessment based on pharmacokinetics."

Antioxidants and Redox Signaling 19(17): 2141-2156. 
Pascual, J. M., D. Wang, B. Lecumberri, H. Yang, X. Mao, R. Yang and D. C. De Vivo (2004). "GLUT1 deficiency and other glucose transporter diseases." European Journal of Endocrinology 150(5): 627-633. Patak, P., H. S. Willenberg and S. R. Bornstein (2004). "Vitamin C is an important cofactor for both adrenal cortex and adrenal medulla." Endocrine Research 30(4): 871-875.

Paxinos, G. and C. Watson (1998). The Rat Brain in Stereotaxic Coordinates. San Diego: Academic Press.

Pierson, H. F. and G. G. Meadows (1983). "Sodium Ascorbate Enhancement of Carbidopa-Levodopa Methyl Ester Antitumor Activity against Pigmented B16 Melanoma." Cancer Research 43(5): 2047-2051. Pilger, A. and H. W. Rudiger (2006). "8-Hydroxy-2'-deoxyguanosine as a marker of oxidative DNA damage related to occupational and environmental exposures." International Archives of Occupational and Environmental Health 80(1): 1-15.

Plautz, G. E., J. E. Touhalisky and S. Shu (1997). "Treatment of Murine Gliomas by Adoptive Transfer of ex Vivo Activated Tumor-Draining Lymph Node Cells." Cellular Immunology 178(2): 101-107. Podhorecka, M., A. Skladanowski and P. Bozko (2010). "H2AX Phosphorylation: Its Role in DNA Damage Response and Cancer Therapy." Journal of Nucleic Acids 2010(Aug 3). pii: 920161.

Poljšak, B., Z. Gazdag, Š. Jenko-Brinovec, Š. Fujs, M. Pesti, J. Bélagyi, S. Plesničar and P. Raspor (2005). "Pro-oxidative vs antioxidative properties of ascorbic acid in chromium(VI)-induced damage: an in vivo and in vitro approach." Journal of Applied Toxicology 25(6): 535-548.

Pollock, J. I. and R. J. Mullin (1987). "Vitamin C biosynthesis in prosimians: Evidence for the anthropoid affinity of Tarsius." American Journal of Physical Anthropology 73(1): 65-70.

Porter, A. (2012). "A Dead End: A Review of Glioblastoma Multiforme." Eukaryon 8(March): 64-68.

Pouliliou, S. and M. I. Koukourakis (2014). "Gamma histone 2AX (gamma$\mathrm{H} 2 \mathrm{AX}$ )as a predictive tool in radiation oncology." Biomarkers 19(3): 167180.

Rebouche, C. J. (1991). "Ascorbic acid and carnitine biosynthesis." The American Journal of Clinical Nutrition 54(6): 1147S-1152S.

Redon, C., D. Pilch, E. Rogakou, O. Sedelnikova, K. Newrock and W. Bonner (2002). "Histone H2A variants H2AX and H2AZ." Current Opinion in Genetics \& Development 12(2): 162-169.

Reppert, E., J. Donegan and L. E. Hines (1951). "Ascorbic Acid and the Hyaluronidase Hyaluronic Acid Reaction." Experimental Biology and Medicine 77(2): 318-320.

Research Services Branch, National Institute of Mental Health and National Institute of Neurological Disorders and Stroke. "Analyse Menu." Retrieved 2 June 2015, 2015, from

http://rsb.info.nih.gov/ij/docs/menus/analyze.html.

Richards, E. (1988). "The Politics of Therapeutic Evaluation: The Vitamin C and Cancer Controversy." Social Studies of Science 18(4): 653-701. 
Riordan, N. H., H. D. Riordan, X. Meng, Y. Li and J. A. Jackson (1995). "Intravenous Ascorbate as a Tumor Cytotoxic Chemotherapeutic Agent." Medical Hypotheses 44: 207-213.

Rivas, C. I., F. A. Zuniga, A. Salas-Burgos, L. Mardones, V. Ormazabal and J. C. Vera (2008). "Vitamin C transporters." Journal of Physiology and Biochemistry 64(4): 357-375.

Rogakou, E. P., D. R. Pilch, A. H. Orr, V. S. Ivanova and W. M. Bonner (1998). "DNA double-stranded breaks induce histone H2AX phosphorylation on serine 139." The Journal of Biological Chemistry 273(10): 5858-5868.

Rose, R. C. and A. M. Bode (1993). "Biology of free radical scavengers: an evaluation of ascorbate." The FASEB Journal 7: 1135-1142.

Ruben, J. D., M. Dally, M. Bailey, R. Smith, C. A. McLean and P. Fedele (2006). "Cerebral radiation necrosis: incidence, outcomes, and risk factors with emphasis on radiation parameters and chemotherapy." International Journal of Radiation Oncology*Biology*Physics 65(2): 499-508.

Rumsey, S. C., O. Kwon, G. W. Xu, C. F. Burant, I. Simpson and M. Levine (1997). "Glucose Transporter Isoforms GLUT1 and GLUT3 Transport Dehydroascorbic Acid." The Journal of Biological Chemistry 272(30): 18982-18989.

Salvati, M., M. Artico, R. Caruso, G. Rocchi, E. R. Orlando and F. Nucci (1991). "A Report on Radiation Induced Gliomas." Cancer 67(2): 392-397. Savini, I., A. Rossi, C. Pierro, L. Avigliano and M. V. Catani (2008). "SVCT1 and SVCT2: key proteins for vitamin C uptake." Amino Acids 34(3): 347355.

Seligman, A. M. and M. J. Shear (1939). "Studies in Carcinogenesis: VIII. Experimental Production of Brain Tumors in Mice with Methylcholanthrene." American Journal of Cancer Research 37(3): 364395.

Selyanin, M. A., P. Y. Boykov, V. N. Khabarov and F. Polyak (2015). "The History of Hyaluronic Acid Discovery, Foundational Research and Initial Use." In: Selyanin, M. A., Boykov, P. Y., Khabarov, V. N. and Polyak, F. (eds): Hyaluronic Acid: Production, Properties, Application in Biology and Medicine. West Sussex: John Wiley \& Sons, Ltd: pgs 1-8.

Sen, C. K. and L. Packer (1996). "Antioxidant and redox regulation of gene transcription." The FASEB Journal 10(7): 709-720.

Sheline, G. E., W. M. Wara and V. Smith (1980). "Therapeutic irradiation and brain injury." International Journal of Radiation

Oncology*Biology*Physics 6(9): 1215-1228.

Shintani, T., N. Hayakawa, M. H. M. Sumida, K. Kurisu, S. Oki, Y. Kodama, H. Kajikawa, K. Inai and N. Kamada (1999). "High Incidence of Meningioma among Hiroshima Atomic Bomb Survivors." Journal of Radiation Research 40(1): 49-57.

Simpson, J. R., J. Horton, C. Scott, W. J. Curran, P. Rubin, J. Fischbach, S. Isaacson, M. Rotman, S. O. Asbell, J. S. Nelson, A. S. Weinstein and D. F. Nelson (1993). "Influence of location and extent of surgical resection on survival of patients with glioblastoma multiforme: Results of three 
consecutive radiation therapy oncology group (RTOG) clinical trials." International Journal of Radiation Oncology*Biology*Physics 26(2): 239244.

Singh, N. P., M. T. McCoy, R. R. Tice and E. L. Schneider (1988). "A simple technique for quantitation of low levels of DNA damage in individual cells." Experimental Cell Research 175(1): 184-191.

Siu, A., J. J. Wind, J. B. Iorgulescu, T. A. Chan, Y. Yamada and J. H. Sherman (2012). "Radiation necrosis following treatment of high grade glioma-a review of the literature and current understanding." Acta Neurochirurgica 154(2): 191-201.

Sonntag, C. V. (2006). Free-Radical-Induced DNA Damage and Its Repair: A Chemical Perspective. Berlin: Springer-Verlag Berlin Heidelberg.

Speit, G. and A. Hartmann (2006). The Comet Assay. DNA Repair Protocols. D. S. Henderson. Totowa, New Jersey, Humana Press. 314: 275-286.

Spickenreither, M., S. Braun, G. Bernhardt, S. Dove and A. Buschauer (2006). "Novel 6-O-acylated vitamin C derivatives as hyaluronidase inhibitors with selectivity for bacterial lyases." Bioorganic \& Medicinal Chemistry Letters 16(20): 5313-5316.

Spinelli, V., O. Chinot, C. Cabaniols, R. Giorgi, P. Alla and M. P. LehucherMichel (2010). "Occupational and environmental risk factors for brain cancer: a pilot case-control study in France." La Presse Médicale 39(2): e35-44.

Stupp, R., W. P. Mason, M. J. van den Bent, M. Weller, B. Fisher, M. J. B. Taphoorn, K. Belanger, A. A. Brandes, C. Marosi, U. Bogdahn, J.

Curschmann, R. C. Janzer, S. K. Ludwin, T. Gorlia, A. Allgeier, D. Lacombe, J. G. Cairncross, E. Eisenhauer, R. O. Mirimanoff, D. Van Den Weyngaert, S. Kaendler, P. Krauseneck, N. Vinolas, S. Villa, R. E. Wurm, M. H. B. Maillot, F. Spagnolli, G. Kantor, J. P. Malhaire, L. Renard, O. De Witte, L. Scandolaro, C. J. Vecht, P. Maingon, J. Lutterbach, A. Kobierska, M. Bolla, R. Souchon, C. Mitine, T. Tzuk-Shina, A. Kuten, G. Haferkamp, J. de Greve, F. Priou, J. Menten, I. Rutten, P. Clavere, A. Malmstrom, B. Jancar, E. Newlands, K. Pigott, A. Twijnstra, O. Chinot, M. Reni, A. Boiardi, M. Fabbro, M. Campone, J. Bozzino, M. Frenay, J. Gijtenbeek, A. A. Brandes, J. Y. Delattre, U. Bogdahn, U. De Paula, M. J. van den Bent, C. Hanzen, G. Pavanato, S. Schraub, R. Pfeffer, R. Soffietti, M. Weller, R. D. Kortmann, M. Taphoorn, J. L. Torrecilla, C. Marosi, W. Grisold, P. Huget, P. Forsyth, D. Fulton, S. Kirby, R. Wong, D. Fenton, B. Fisher, G. Cairncross, P. Whitlock, K. Belanger, S. Burdette-Radoux, S. Gertler, S. Saunders, K. Laing, J. Siddiqui, L. A. Martin, S. Gulavita, J. Perry, W. Mason, B. Thiessen, H. Pai, Z. Y. Alam, D. Eisenstat, W. Mingrone, S. Hofer, G. Pesce, J. Curschmann, P. Y. Dietrich, R. Stupp, R. O. Mirimanoff, P. Thum, B. Baumert, G. Ryan and The European Organisation for Research and Treatment of Cancer (2005). "Radiotherapy plus concomitant and adjuvant temozolomide for glioblastoma." New England Journal of Medicine 352(10): 987-996.

Szatmari, T., K. Lumniczky, S. Desaknai, S. Trajcevski, E. J. Hidvegi, H. Hamada and G. Safrany (2006). "Detailed characterization of the mouse 
glioma 261 tumor model for experimental glioblastoma therapy." Cancer Science 97(6): 546-553.

Tahiliani, M., K. P. Koh, Y. Shen, W. A. Pastor, H. Bandukwala, Y. Brudno, S. Agarwal, L. M. Iyer, D. R. Liu, L. Aravind and A. Rao (2009). "Conversion of 5-Methylcytosine to 5-Hydroxymethylcytosine in Mammalian DNA by MLL Partner TET1." Science 324(5929): 930-935.

Takahashi, A. and T. Ohnishi (2005). "Does gammaH2AX foci formation depend on the presence of DNA double strand breaks?" Cancer Letters 229(2): 171-179.

Tanaka, T., A. Kurose, X. Huang, F. Traganos, W. Dai and Z. Darzynkiewicz (2006). "Extent of constitutive histone H2AX phosphorylation on Ser-139 varies in cells with different TP53 status." Cell Proliferation 39(4): 313-323. Thomas, C. G., P. E. Vezyraki, V. P. Kalfakakou and A. M. Evangelou (2005). "Vitamin C transiently arrests cancer cell cycle progression in S phase and $\mathrm{G} 2 / \mathrm{M}$ boundary by modulating the kinetics of activation and the subcellular localization of Cdc25C phosphatase." Journal of Cellular Physiology 205(2): 310-318.

Tice, R. R., E. Agurell, D. Anderson, B. Burlinson, A. Hartmann, H. Kobayashi, Y. Miyamae, E. Rojas, J. C. Ryu and Y. F. Sasaki (2000). "Single cell gel/comet assay: guidelines for in vitro and in vivo genetic toxicology testing." Environmental and Molecular Mutagenesis 35(3): 206-221. Tice, R. R. and G. H. Strauss (1995). "The single cell gel electrophoresis/comet assay: a potential tool for detecting radiationinduced DNA damage in humans." Stem Cells 13(Suppl 1): 207-214. Tsao, C. S. (1991). "Inhibiting effect of ascorbic acid on the growth of human mammary tumour xenografts." The American Journal of Clinical Nutrition 54(6): 1274S-1280S.

Tsukaguchi, H., T. Tokui, B. Mackenzie, U. V. Berger, X.-Z. Chen, Y. Wang, R. F. Brubaker and M. A. Hediger (1999). "A family of mammalian Na+dependent L-ascorbic acid transporters." Nature 399(6731): 70-75.

Ullah, M. F., H. Y. Khan, H. Zubair, U. Shamim and S. M. Hadi (2010). "The antioxidant ascorbic acid mobilizes nuclear copper leading to a prooxidant breakage of cellular DNA: implications for chemotherapeutic action against cancer." Cancer Chemotherapy and Pharmacology 67(1): 103-110.

University of North Carolina School of Medicine. (2015).

"Comet_Assay.txt." Retrieved 2 June 2015, 2015, from http://www.med.unc.edu/microscopy/files/resources/Comet_Assay.txt/vi ew.

Urbanska, K., J. Sokolowska, M. Szmidt and P. Sysa (2014). "Glioblastoma multiforme - an overview." Contemporary Oncology 18(5): 307-312. Valavanidis, A., T. Vlachogianni and C. Fiotakis (2009). "8-hydroxy-2' deoxyguanosine (8-OHdG): A critical biomarker of oxidative stress and carcinogenesis." Journal of Environmental Science and Health. Part C, Environmental carcinogenesis \& ecotoxicology reviews 27(2): 120-139. Valavanidis, A., T. Vlachogianni and C. Fiotakis (2009). "8-hydroxy-2' deoxyguanosine (8-OHdG): A critical biomarker of oxidative stress and 
carcinogenesis." Journal of Environmental Science and Health. Part $C_{1}$ Environmental Carcinogenesis \& Ecotoxicology Reviews 27(2): 120-139. Varga, J. M. and L. Airoldi (1983). "Inhibition of transplantable melanoma tumor development in mice by prophylactic administration of $\mathrm{Ca}$ ascorbate." Life Sciences 32(14): 1559-1564.

Varjovi, M. B., M. Valizadeh and A. Bandehagh (2015). "Primary Antioxidant Enzymes and their Important Role in Oxidative Stress in Plants and Mammalian." Biological Forum 7(1): 148-154.

Vera, J. C., C. I. Rivas, J. Fischbarg and D. W. Golde (1993). "Mammalian facilitative hexose transporters mediate the transport of dehydroascorbic acid." Nature 364(6432):79-82.

Verhaak, R. G., K. A. Hoadley, E. Purdom, V. Wang, Y. Qi, M. D. Wilkerson, C. R. Miller, L. Ding, T. Golub, J. P. Mesirov, G. Alexe, M. Lawrence, M. O'Kelly, P. Tamayo, B. A. Weir, S. Gabriel, W. Winckler, S. Gupta, L. Jakkula, H. S. Feiler, J. G. Hodgson, C. D. James, J. N. Sarkaria, C. Brennan, A. Kahn, P. T. Spellman, R. K. Wilson, T. P. Speed, J. W. Gray, M. Meyerson, G. Getz, C. M. Perou, D. N. Hayes and The Cancer Genome Atlas Research Network (2010). "An integrated genomic analysis identifies clinically relevant subtypes of glioblastoma characterized by abnormalities in PDGFRA, IDH1, EGFR, and NF1." Cancer Cell 17(1): 98-110.

Vonk, L. A., B. Z. Doulabi, C. L. Huang, M. N. Helder, V. Everts and R. A. Bank (2010). "Endoplasmic reticulum stress inhibits collagen synthesis independent of collagen-modifying enzymes in different chondrocyte populations and dermal fibroblasts." Biochemistry and Cell Biology 88(3): 539-552.

Walker, M. D., J. Eben Alexander, W. E. Hunt, C. S. MacCarty, J. M. Stephen Mahaley, J. John Mealey, H. A. Norrell, G. Owens, J. Ransohoff, C. B. Wilson, E. A. Gehan and T. A. Strike (1978). "Evaluation of BCNU and/or radiotherapy in the treatment of anaplastic gliomas." Journal of Neurosurgery 49(3): 333-343.

Wang, Y., T. A. Russo, O. Kwon, S. Chanock, S. C. Rumsey and M. Levine (1997). "Ascorbate recycling in human neutrophils: Induction by bacteria." Proceedings of the National Academy of Sciences of the United States of America 94(25): 13816-13819.

Wardman, P. and L. P. Candeias (1996). "Fenton chemistry: an introduction." Radiation Research 145(5): 523-531.

Wen, P. Y., H. A. Fine, P. M. Black, D. C. Shrieve, E. A. III and J. S. Loeffler (1995). "High-grade Astrocytomas." In: Wen, P.Y. and Black, P.M. (eds): Brain Tumors in Adults. Neurologic Clinics of North America, W.B Saunders Co: Philadelphia, 13(4): 875-900.

Wen, P. Y. and S. Kesari (2008). "Malignant Gliomas in Adults." New England Journal of Medicine 359(5): 492-507.

Withers, H. R. (1992). "Biological basis of radiation therapy for cancer." The Lancet 339(8786): 156-159.

Woods, R. J. and A. K. Pikaev (1994). Applied Radiation Chemistry: Radiation Processing. New York: Wiley. 
World Cancer Research Fund, American Institute for Cancer Research (2007). Food, Nutrition, Physical Activity, and the Prevention of Cancer: a Global Perspective. Washington, DC: AICR.

Wrensch, M., Y. Minn, T. Chew, M. Bondy and M. S. Berger (2002).

"Epidemiology of primary brain tumors: Current concepts and review of the literature." Neuro-Oncology 4(4):278-99.

$\mathrm{Wu}, \mathrm{H}$. and Y. Zhang (2011). "Mechanisms and functions of Tet proteinmediated 5-methylcytosine oxidation." Genes and Development 25(23): 2436-2452.

Xia, C., Q. Meng, L. Z. Liu, Y. Rojanasakul, X. R. Wang and B. H. Jiang (2007). "Reactive oxygen species regulate angiogenesis and tumor growth through vascular endothelial growth factor." Cancer Research 67(22): 1082310830.

Yen, G.-C., P.-D. Duh and H.-L. Tsai (2002). "Antioxidant and pro-oxidant properties of ascorbic acid and gallic acid." Food Chemistry 79(3): 307-313. Yuan, J., R. Adamski and J. Chen (2010). "Focus on Histone variant H2AX: to be or not to be." FEBS letters 584(17): 3717-3724.

Zeljezic, D., M. Mladinic, N. Kopjar and A. H. Radulovic (2015). "Evaluation of genome damage in subjects occupationally exposed to possible carcinogens." Toxicology and Industrial Health. 2015(Feb 4). pii: 0748233714568478.

Zhang, L., G. Huang, X. Li, Y. Zhang, Y. Jiang, J. Shen, J. Liu, Q. Wang, J. Zhu, X. Feng, J. Dong and C. Qian (2013). "Hypoxia induces epithelialmesenchymal transition via activation of SNAI1 by hypoxia-inducible factor -1alpha in hepatocellular carcinoma." BMC Cancer 13(1): 1-9.

Zhao, D. Y., K. M. Jacobs, D. E. Hallahan and D. Thotala (2015). "Silencing Egr1 Attenuates Radiation-Induced Apoptosis in Normal Tissues while Killing Cancer Cells and Delaying Tumor Growth." Molecular Cancer Therapeutics 14(10): 2343-2352.

Zhao, L., Y. Quan, J. Wang, F. Wang, Y. Zheng and A. Zhou (2015). "Vitamin $C$ inhibit the proliferation, migration and epithelial-mesenchymaltransition of lens epithelial cells by destabilizing HIF-1 $\alpha . "$ International Journal of Clinical and Experimental Medicine 8(9): 15155-15163.

Ziello, J. E., I. S. Jovin and Y. Huang (2007). "Hypoxia-Inducible Factor (HIF)1 Regulatory Pathway and its Potential for Therapeutic Intervention in Malignancy and Ischemia." The Yale Journal of Biology and Medicine 80(2): 51-60. 


\section{List of Abbreviations}

BSA

CNS

DHA

DMEM

DNA

DSBs

$\mathrm{EC}_{50}$

EDTA

$\mathrm{EtOH}$

FACS

FRs

$\mathrm{FSC}(\mathrm{A} / \mathrm{H})$

GBM

GL261

GLUT

Gy

IF

IP

$\mathrm{M}$

$\mu \mathrm{M}$

$\mathrm{mM}$

$\mathrm{NaCl}$

PBS

ROS

RPMI-1640

SEM

SSBs

$\mathrm{SSC}(-\mathrm{A} / \mathrm{H})$

SVCT

TBE

TBS

TBST

TE

WHO
Bovine serum albumin

Central Nervous System

Dehydroascorbic Acid

Dulbecco's Modified Eagle Medium

Deoxyribonucleic Acid

Double Strand Breaks

Effective Concentration 50\%

Ethylenediaminetetraacetic acid

Ethanol

Fluorescence Activated Cell Sorting (flow cytometry)

Free Radicals

Forward Scatter (Area/Height)

Glioblastoma Multiforme

Glioma 261

Glucose Transporter

Gray

Immunofluorescence

Intraperitoneal

Molar

Micromolar

Millimolar

Sodium Chloride

Dulbecco's Phosphate Buffer Saline

Reactive Oxygen Species

Roswell Park Memorial Institute medium 1640

Standard error of mean

Single Strand Breaks

Side Scatter (Area/Height)

Sodium Dependent Vitamin C Transporter

Tris Boric acid EDTA buffer

Tris buffered saline

Tris buffered saline with Tween-20

Trypsin EDTA

World Health Organisation 